University of Rhode Island

DigitalCommons@URI

Open Access Dissertations

1983

\title{
Groundwater Flow Simulation by a Stochastic Representation of Soil
}

Paul Bruce Aldinger

University of Rhode Island

Follow this and additional works at: https://digitalcommons.uri.edu/oa_diss

\section{Recommended Citation}

Aldinger, Paul Bruce, "Groundwater Flow Simulation by a Stochastic Representation of Soil" (1983). Open Access Dissertations. Paper 546.

https://digitalcommons.uri.edu/oa_diss/546

This Dissertation is brought to you for free and open access by DigitalCommons@URI. It has been accepted for inclusion in Open Access Dissertations by an authorized administrator of DigitalCommons@URI. For more information, please contact digitalcommons-group@uri.edu. 
GROUNDWATER FLOW SIMULATION BY A STOCHASTIC REPRESENTATION OF SOIL

BY

PAUL BRUCE ALDINGER

A THESIS SUBMITTED IN PARTIAL FULFILLMENT OF THE REQUIREMENTS FOR THE DEGREE OF DOCTOR OF PHILOSOPHY

IN

CIVIL AND ENVIRONMENTAL ENGINEERING UNIVERSITY OF RHODE ISLAND 


\section{DOCTOR OF PHILOSOPHY DISSERTATION}

OF

PAUL BRUCE ALDINGER

\section{Approved:}

Dissertation Committee

Major Professor
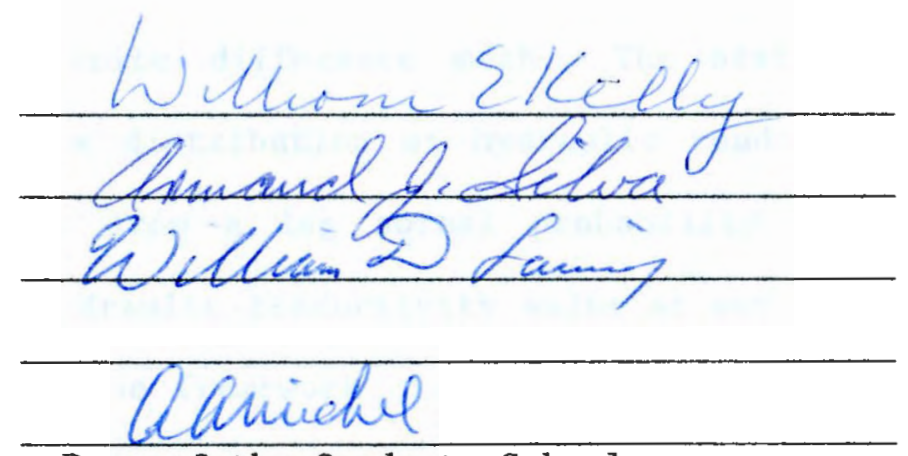

Dean of the Graduate School

\section{UNIVERSITY OF RHODE ISLAND}

1983 
ABSTRACT

A Monte Carlo technique is utilized to incorporate the uncertainty in media characteristics to the solution of a groundwater flow problem. This technique involves the repetitive solution of a significant number of equiprobable representations of the soil medium. Probability and statistics are utilized to model the soil parameters and study the significance of the output.

An existing computer code was adapted and significantly modified to allow characterization of the media's hydraulic conductivity (permeability) as autocorrelated and statistically homogeneous. A first order nearest neighbor model was selected to affect the autocorrelation of this parameter within the finite difference mesh. The statistical homogeneity considers that the distribution of hydraulic conductivity values within the mesh comes from a log normal probability density function. The selection of hydraulic conductivity value at any mode of the mesh is stochastic within the framework of the autocorrelation and statistical homogeneity of the mesh aggregate.

The computer code takes the stochastically generated hydraulic conductivity field and the boundary conditions and utilizing an iterative alternating direct implicit solution determines the hydraulic head values at each mode and flow rate through the medium. An array of these results are produced for each of the equiprobable representations of the soil medium. 
Mass transport through the region is simulated as a combination of advection and a stochastic simulation of microscopic or particle scale dispersion. A water particle is released from a preselected location along the upgradient boundary. The particle moves toward the downgradient boundary under the influence of advective forces caused by the differences in hydraulic head and the stochastic simulation of microscopic dispersion. This simulation of microscopic dispersion displaces the particle parallel to and perpendicular to the advective transport direction based on laboratory scale dispersivities. The computer code establishes arrays for the particle location at a predetermined time after start as well as the location along the downgradient boundary and total travel time upon completion of transit of the region.

Uniform flow results in most of the regions considered although some alternate configurations were considered. An effective hydraulic conductivity is calculated on the basis of flow rate. After application of a shape factor this value was found to be slightly less but closest to the geometric mean of the hydraulic conductivity distribution thus confirming earlier work. An alternative effective hydraulic conductivity calculated on the basis of travel time was also determined. This value was generally less than the other effective hydraulic conductivity value but again after application of a shape factor the value was best estimated by the geometric mean. These results suggest that the mean flow rate and mean travel time may be estimated by the use of the shape factor from a flow net solution or the method of fragments and the geometric means of the hydraulic conductivity. 
The results of the simulations indicate that macroscopic or field scale longitudinal and lateral dispersion is significantly affected by the standard deviation of the hydraulic conductivity distribution. Region size, hydraulic gradient and time interval were found to cause lesser effects.

The techniques utilized provide a means to develop confidence in the output. The effects of the variations in parameters become evident from a review of the results of the equiprobable results. Confidence limits may even be developed in the output where the characteristics of known probability density functions may be utilized. Example problems are presented where confidence limits on the estimates of travel time are developed for the conditions considered. 


\section{ACKNOWLEDGEMENTS}

I would like to express my appreciation to Professors Kelly, Silva and Lawing for their guidance and assistance on this thesis. I am particularly appreciative of the assistance of my major professor for his assistance over the many years at the University particularly this last year when he assisted me from his new assignment in Nebraska. During the past years I have been supported by CE Maguire, Inc. and last year by a University Fellowship. This support is appreciated and gratefully acknowledged. Also I would like to thank Terry Tomasian, Barbara Fetta, Dora Leal and Pat Wittaker for their assistance in typing the manuscript and Lou Mazzuchelli for his assistance with the graphics.

Finally I would like to acknowledge the support given me by my wife, Mary Lou and my children who have sacrificed immeasurably over these past seven years. 
TABLE OF CONTENTS

Page

Approval Page

Thesis Abstract $\quad$ ii

Acknowledgements $\quad$ v

Table of Contents vi

List of Tables $\quad$ viii

List of Figures $\quad$ ix

$\begin{array}{ll}\text { Preface } & \text { xv }\end{array}$

Section I - Groundwater Flow in a Stochastic Medium: Model Development

$\begin{array}{ll}\text { Abstract } & 1\end{array}$

Introduction 1

$\begin{array}{ll}\text { Background } & 5\end{array}$

$\begin{array}{ll}\text { Model Input Development } & 10\end{array}$

$\begin{array}{ll}\text { Mass Transport Simulation } & 18\end{array}$

$\begin{array}{lr}\text { Results and Discussion } & 21\end{array}$

$\begin{array}{ll}\text { Conclusions } & 64\end{array}$

Appendix I - Reference $\quad 66$

Appendix II - Notation 72

Section II - Groundwater Flow in a Stochastic Medium: Applications of Computer Model

$\begin{array}{ll}\text { Abstract } & 76\end{array}$

$\begin{array}{ll}\text { Introduction } & 76\end{array}$

$\begin{array}{ll}\text { Background } & 78\end{array}$ 
Flow Beneath Dike Example $\quad 80$

Groundwater in Vicinity of a Landfill Example $\quad 100$

$\begin{array}{lr}\text { Conclusions } & 109\end{array}$

Appendix - References 111

Section III - Appendices

A. Introduction and Review of the Problem 113

B. Details of Methods Used 162

$\begin{array}{ll}\text { C. Bibliography } & 241\end{array}$

D. Computer Programs 252 


\section{LIST OF TABLES}

Page

Section I

Table 1 Summary of Flow Simulations

Section II

Table 1 Summary of Results - Dike Example

Table 2 Summary of Results - Landfill Example

104

Appendix A

A.1 Probability Density Functions for Several Soil Properties

Appendix B

Table 1 Summary of Flow Simulations 


\section{LIST OF FIGURES}

Page

Section I

Figure 1 General Plan of Flow Region

Figure 2 Probability Density Function for Hydraulic 11 Conductivity

Figure $3 \quad$ Correlation of Hydraulic Conductivity 13

Figure $4 \quad$ Region of Mass Transport 24

Figure 5 Correlation of Effective Hydraulic Conduc 26

Figure $6 \quad$ Alternate Boundary Conditions 30

Figure $7 \quad$ Correlation Coefficient Versus Lag Number 33

Figure $8 \quad$ Integral Scale Versus Region Size

Figure 9 Cumulative Mean Values of Mean and Standard 37 Deviation of Hydraulic Conductivity Versus Simulation Number

Figure 10 Cumulative Mean Values of Mean and Standard 40 Deviation of Travel Times Versus Particle Number

Figure 11 Cumulative Mean of Mean and Standard Devi ation of Ending $Y$ Location Versus Particle Number

Figure $12 \quad$ Typical Breakthrough Curve 44

Figure 13 Mean, Standard Deviation and Variance of Hydraulic Head Versus Node Number

Figure 14 Cumulative Mean of the Head Versus Sim ulation Number

Figure 15 Maximum Value of Normalized Standard Devi ation of Head Versus Region Size

Figure 16 Maximum Value of Standard Deviation of Head Versus Hydraulic Gradient

Figure 17 Maximum Value of Standard Deviation of Head Versus Standard Deviation of Hydraulic Conductivity 
Figure 18 Macroscopic Dispersivity Versus Region Size

Figure 19 Macroscopic Dispersivity Versus Hydraulic Gradient

Figure 20 Macroscopic Dispersivity Versus Time Inter-

Figure 21 Macroscopic Dispersivity Versus Standard

Figure 22 Typical Output on Flow and Travel Time

Figure 23 Hypothetical Breakthrough Curve with Confidence Limits

\section{Section II}

Figure 1 Relationship Between Grain Size, Standard Penetration Test and Porosity

Figure 2 Big River Reservoir Project Area

$\begin{array}{llr}\text { Figure } 3 & \text { Division Street Dike Vicinity Plan } & 84\end{array}$

Figure 4 Generalized Hydrogeologic Profile Number 1

Figure $5 \quad$ Generalized Hydrogeologic Profile Number $2 \quad 87$

Figure $6 \quad$ Computer Simulated Profile Number 1

Figure $7 \quad$ Computer Simulated Profile Number $2 \quad 90$

Figure $8 \quad X$ and Y Coordinates After 191 Days 94

Figure 9 Starting X Coordinate Versus Y Coordinate 95

Figure 10 Total Travel Time Versus Starting X Coor- 96

Figure 11 Cumulative Mean of the Mean and Lag 3 Standard Deviation of Effective Hydraulic Conductivity Versis Simulation Number Profile 1

Figure 12 Cumulative Mean of the Mean and Lag 3 Conductivity Versus Simulation Number Profile 2 
Figure 13 Landfill Vicinity Plan

Figure 14 Computer Simulated Landfill Profile

Figure 15 Particle Location After 191 Days and Start-

Figure 16 Total Travel Time Versus Starting X Coor107 dinate

Figure 17 Statistical Summary on Travel Time

Appendix A

Figure A.1 Flow Region Representation

Figure A.2 General Flow Region

123

Figure A.3 Variability of Soil Engineering Properties

125

Figure A.4 Empirical Relationship between Grain Size

Figure A.5 Other Empirical Relationship between Grain Size and Hydraulic Conductivity

Figure A.6 Probability Density Function for the Normal Distribution

135

Figure A.7 Probability Density Function for the Log

Normal Distribution

Figure A.8 Example of Use of Histogram, Probability

Density Function and Normal Probability Paper

Figure A.9 Possible Probability Density Functions for Hydraulic Conductivity

Figure A.10 Autocorrelation Decay with Distance

Figure A.11 Wide Band Random Noise Process

Figure A.12 Van Marke's Stochastic Representation of 155

Figure A.13 Tracer Experiment Layout 
Appendix B

Figure B.1 General Plan of Region 163

Figure B.2 Finite Difference Representation $\quad 168$

Figure B.3 7 By 7 Example of Region Representation 171

Figure B.4 Weighting Matrix for 7 By 7 Region 172

Figure B.5 Schematic View of Interior Node of Fimte 181

$\begin{array}{lll}\text { Figure B.6 Velocity Vector Diagram } & 185\end{array}$

Figure B.7 General Flow Region 186

Figure B.8 Computer Time Versus Number of Nodes $\quad 192$

$\begin{array}{llr}\text { Figure B.9 Region of Mass Transport } & 194\end{array}$

Figure B.10 Correlation of Effective Hydraulic Conduc- 197 tivities with Geometric Mean of Hydraulic Conductivity

Figure B.11 Alternate Flow Configurations 200

Figure B.12 Hydraulic Conductivity Comparisons 202

Figure B.13 Correlation Coefficient Versus Lag 204

Figure B.14 Comparison of Auto Correlation Coefficients 206

Figure B.15 Effects of Shaving and Stacking of Blocks 207

Figure B.16 Effects of Region Size on Autocorrelation 209

Figure B.17 Effects of Autoregressive Parameters on 210

Figure B.18 Cumulative Mean of the Arithmetic Mean and Lag 3 Standard Deviation of Hydraulic Conductivity Versus Simulation Number

Figure B.19 Cumulative Mean of the Mean and Lag 3 Standard Deviation of Effective Hydraulic Conductivity (Flux) Versus Simulation Number 
Figure B.20 Cumulative Mean of the Mean and Standard Deviation of Effective Hydraulic Conductivity (Travel Time) Versus Simulation Number

Figure B.21 Cumulative Mean of the Mean and Lag 3 Standard Deviation of Travel Time Versus Particle Number

Figure B.22 Cumulative Mean of the Mean and Lag 3 Standard Deviation of Normalized Ending $y$ Location

Figure B.23 Simulation Breakthrough Curve

Figure B.24 Mean, Standard Deviation and Variance of Head Versus Node Number

Figure B.25 Cumulative Mean of the Mean and Lag 3 Stan224 dard Deviation of Head at Interior Node Versus Simulation Number

Figure B.26 Maximum Value of Normalized Standard Deviation of Hydraulic Head Versus Region Size

Figure B.27 Maximum Value of Standard Deviation of Head Versus Hydraulic Gradient

Figure B.28 Maximum Value of Standard Deviation of Head Versus Standard Deviation of Hydraulic Connductivity

Figure B.29 Macroscopic Dispersivity Versus Region Size

Figure B.30 Macroscopic Dispersivity Versus Hydraulic 231 Gradient

Figure B.31 Macroscopic Dispersivity Versus Time Inter232 val

Figure B.32 Macroscopic Dispersivity Versus the Stan234 dard Deviation of Hydraulic Conductivity

Figure B.33 Flow Region and Typical Output of Effective 236 Hydraulic Conductivity (Flux) and Travel Time

Figure B.34 Hypothetical Breakthrough Curve 
Figure B.35 Macroscopic Dispersion Coefficient and Dispersivity Versus Mean Velocity and Dispersivity Versus Standard Deviation of Hydraulic Conductivity 


\section{PREFACE}

This thesis is presented in the manuscript format. Section I, Groundwater Flow in a Stochastic Medimum: Model Development and Section II, Groundwater Flow in a Stochastic Medium: Application of Computer Model, will be submitted for publication in the Journal of the Geotechnical Division of the ASCE. Both manuscripts are presented in the format of that journal.

Details of the methods employed including the computer program as well as additional ancillary information are presented in the Appendices in Section III. 


\author{
SECTION I \\ GROUNDWATER FLOW IN A STOCHASTIC MEDIUM: \\ MODEL DEVELOPMENT \\ by Paul Bruce Aldinger, ${ }^{1}$ M. ASCE
}

\begin{abstract}
A computer model is developed to simulate groundwater flow in a stochastic porous medium. The hydraulic conductixity (permeability) of the medium is simulated as made up of statistically homogeneous strata which are internally correlated by a two dimensional nearest neighbor model. Dispersion is simulated as a combination of advective transport, and a stochastic simulation of microscopic dispersion. The model utilizes a Monte Carlo technique of repetitively solving equiprobable representations of the medium. Output consisting of hydraulic head, flow rate, travel time and dispersion is then analyzed to allow a measure of significance. A shape factor is developed which may be utilized to calculate an equivalent value of hydraulic conductivity for simplified computation of the mean flux and travel times.
\end{abstract}

\title{
INTRODUCTION
}

Groundwater flow problems have always been particularly challenging due to their complexity and the uncertainties involved. Increasingly, predictions of flow quantity, direction and/or travel time are necessary to answer questions on risk and safety. This need for establishing the reliability of such predictions has brought investigators from various disciplines within the sciences into this arena. The result is that considerable progress has been achieved in advancing the state of the art in recent years.

${ }^{1}$ Principal Geotechnical Engineer, Department Manager, CE Maguire, Inc., Providence, Rhode Island 
The complexity of these problems is due to many factors. One factor is the difficulty in scaling up and combining the properties of the medium on the microscopic level to the macroscopic scale of the flow region. A related factor is the incomplete knowledge of flow regime caused by the complex conditions during deposition and/or subsequent weathering. Still other factors are the sheer number and range of variables and the limited resources normally available for analysis.

Over the years, numerous techniques have been utilized to solve these groundwater flow problems. Analytical solutions have been developed for many flow situations, however, analytic techniques suffer from a lack of flexibility and the need to make restrictive simplifying assumptions on boundary conditions and media characteristics, thereby limiting their use with many practical problems.

Perhaps the technique most widely used today is that of flow nets (10, 11). Its greatest advantage is its simplicity and ease in use. However, this technique is limited in its ability to vary soil hydraulic conductivity and groundwater surface or free surface.

A similarity with all of these techniques is the lack of flexibility in handling boundary conditions and/or varying soil property characteristics. When these techniques are utilized, the consequences of the required averaging and other approximations are often unknown.

With the recent interest in this area from the multitude of scientific disciplines and with the advancements in computer technology in the last 
decade, major changes in the way these flow problems are analyzed have occurred. Investigators have developed numerical solutions which allow much more latitude in setting boundary conditions and input parameters allowing much more complex and realistic characterizations of flow regimes to be considered.

Numerical solutions are approximate solutions to the flow problem. However, when properly executed they result in solutions within almost any desired level of accuracy. The most common types of numerical solutions in use today are the finite element method (FEM) and the finite difference method (FDM). Either of these methods may be used to solve most of the situations encountered, however, one type may have some advantage over the other for particular problems. A review of these methods may be found elsewhere $(24,35)$.

The FEM and FDM methods have allowed not only more complex boundary conditions and input parameter characterization but also allowed introduction of new types of considerations. Of these, stochastic generation of a correlated hydraulic conductivity distribution to characterize the flow regions, stochastic simulation of microscopic dispersion and the utilization of probability concepts or theory to analyze input and output are major considerations of this work.

The flow region considered in this study is two dimensional. Figure 1 presents the study region with the simplest boundary conditions resulting in predominantly uniform horizontal flow. The flow condition is referred to as uniform flow because flow is largely in one dimension, 

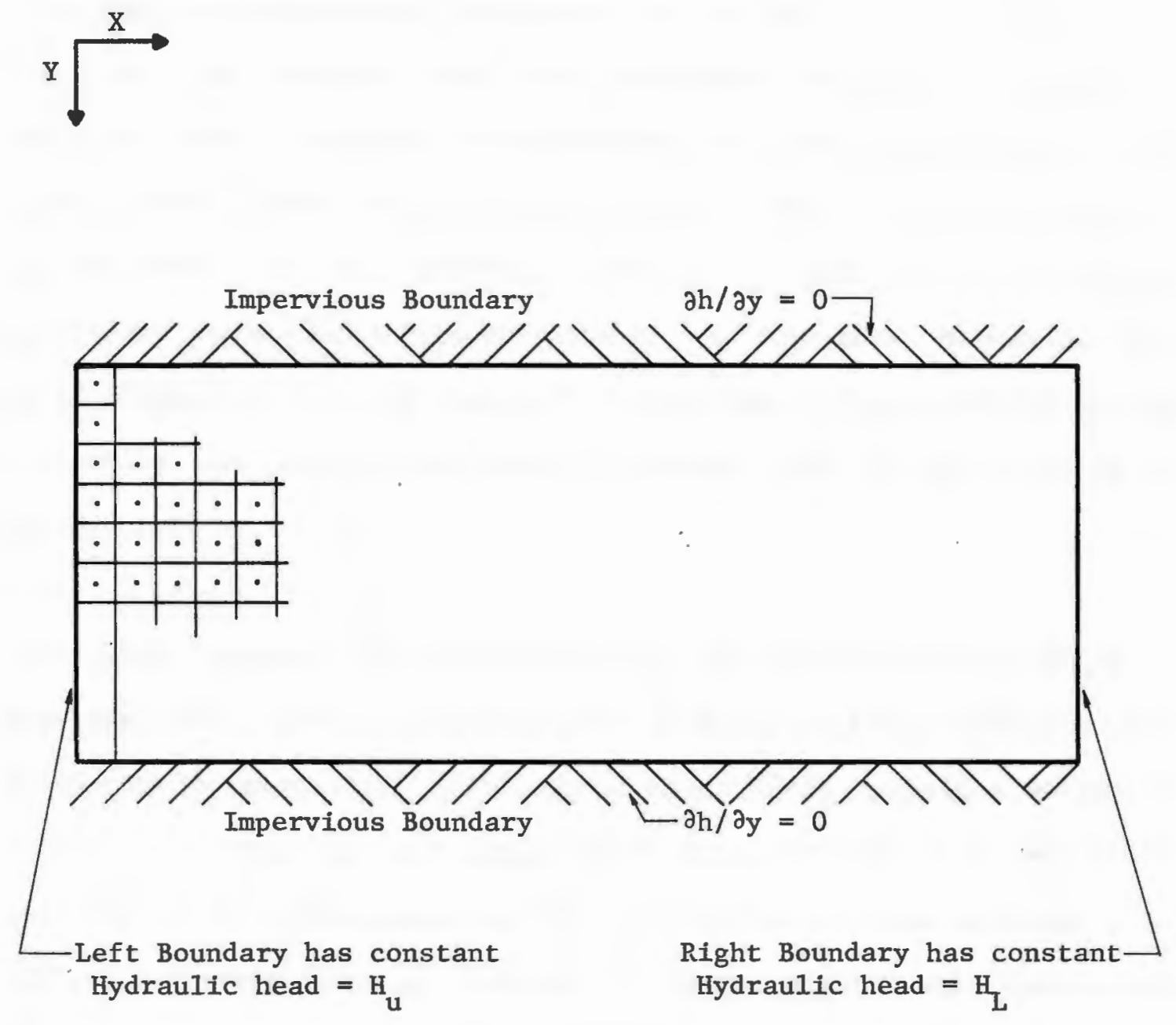

Figure 1 - General Plan of Flow Region 
from the left side boundary to the right side boundary. A numerical solution by the finite difference method for steady state flow is used to determine hydraulic heads and flow within the region.

Water particles within the flow region are subject to advective transport forces and microscopic dispersion due to the pore structure. Water particles are released from the upgradient boundary, subjected to advection and a stochastic representation of microscopic dispersion and their travel across the region monitored. This simulation allows a determination of the relative effects of hydraulic conductivity variations on observed dispersivities. It has been recognized that field dispersivities are generally higher than those measured in the laboratory and researchers have attributed this to the effects of heterogeneities.

This paper presents the background for the methodology developed to simulate water flow in a medium with an autocorrelated hydraulic conductivity (permeability) mesh. By a Monte Carlo technique which involves generating numerous equiprobable distributions, a better understanding of the uncertainty in the predictions of flow quantity, dispersion and travel time is obtained. A companion paper will demonstrate its use to solve two types of real groundwater flow problems. (3)

\section{BACKGROUND}

The soil parameters required to characterize a flow medium are the hydraulic conductivity (permeability), porosity, and the dispersion 
parameters. It should be pointed out that the term hydraulic conductivity is utilized rather that the more common geotechnical engineering term of permeability to avoid confusion with workers in other disciplines. The term permeability is reserved for the medium's flow characteristic without the effects of the pore fluid's characteristics. This is comparable with the work in the geologic and petroleum industries and with similar terminology in such areas as heat, electrical and chemical flow.

The most significant of the soil parameters governing groundwater flow is hydraulic conductivity. The range of values for hydraulic conductivity of normal soil deposits is as much as 13 orders of magnitude. This variation is large for a soil property. By comparison, porosity may only vary between zero and one, unit weights by less than one and dispersivity by two orders of magnitude.

The variation within one single soil type can also be large. A variation of three orders of magnitude within a soil designation, such as sand or silt, is possible. The engineer has, by necessity, to categorize similar soil encountered in soil explorations into various soil strata. The variation in hydraulic conductivity in one soil stratum may be as much as one order of magnitude. This suggests that knowing the order of magnitude of hydraulic conductivity in a soil stratum may be enough and to expect to know the value at one particular point in a soil mass to within one order of magnitude is somewhat presumptive. Indeed we should expect considerable variation within even a "homogeneous" soil. 
It is this potential for wide variation and therefore the implied uncertainty which has led engineers and geologists to turn to probability and statistical techniques to characterize hydraulic conductivity. The value of hydraulic conductivity in situ is considered to be a random variable in this methodology. This implies that the exact value at any point cannot be predicted with certainty a priori. There is uncertainty in any estimate prior to experimentation.

A random variable may be described by a probability density function (PDF) which simply relates the value of a variable with its corresponding probability. A Monte Carlo solution is accomplished by generating a significant number of equally probable representations of the flow medium from the PDF, repetitively solving each for the unknown desired results, and analyzing the results to determine the "most probable" results as well as a measure of the magnitude of variation of that result.

There are several well studied PDF's which have been found to adequately characterize soil properties $(6,20,24)$. The Gaussian or normal distribution is one of the most commonly studies PDF's. This distribution is completely defined by the mean and standard deviation. This distribution has been found to model well those processes which are additive of many processes or random variables. This is the premise of the Central Limit Theorem. An example of a soil property which has been found to follow this PDF is porosity. 
Another PDF which has been found to model some soil random variables is the 108 normal distribution. This distribution represents well those processes which are the result of multiplying other random variables. Hydraulic conductivity has been found to be adequately characterized by a $\log$ normal PDF $(13,29,32)$. A useful identity is that the PDF of the log of a random variable with a lognormal PDF is normal.

The physical processes which result in soil deposits may be quite random and conditions predominating at one time and place will be modified elsewhere. It should be expected, however, to find similarities at adjacent locations when compared to locations separated by some distance. This similarity is often quantified by calculation of the autocorrelation of the random variable.

Techniques to accomplish this spatial autocorrelation include a nearest neighbor model, trend analysis, spectral density and variations of these. The nearest neighbor models develop equations for the dependency of the variable at adjacent locations and solve these equations for model development. This technique is adopted here so that further discussion will follow.

The trend analysis technique was developed at Northwestern University by Krumbein and Whitten $(23,42)$. The technique assumes that the random variable at any point may be characterized as the summation of a trend component (dependent on location) and a random component. Koch and Link (22) present an excellent introduction to this technique. Tabba and Yong (36) have developed a working model and in a companion paper (37) 
demonstrate its application to a real problem. This technique interpolates and extrapolates information on the random variables to all points in the flow media.

The spectral density technique recognizes the Fourier transform pair consisting of the spectral density function of the random variable $S(f)$, and the autocorrelation function, $R(x)$. $S(f)$ describes the frequency information for the process and $R(x)$ the autocorrelation. This approach may describe the variations in soil properties more precisely than the other. However, its inherent complexity and the need for large amounts of data has discouraged its use for real problems in groundwater flow. It's use in solving groundwater flow problems has been demonstrated (4, $16,17,18)$.

The effects of dispersion during advective transport is becoming more critical as interest in tracking movement of pollutants and predicting concentrations in groundwater systems increases. Others have studied the problem of dispersion in groundwater flow stochastically $(17,35$, 41). The first and last papers incorporate microscopic dispersion in addition to advective transport. Smith and Schwartz (35) demonstrate that large scale spatial variations in hydraulic conductivity are the major factor causing macroscopic or field scale dispersion in groundwater flow. Microscopic dispersivity is a minor factor. The assumption of normality for the travel time ensemble has not been confirmed. Gelhar, et al (17) indicated that for long travel distances a constant longitudinal dispersivity should be expected. 
A methodology to include microscopic dispersion was first developed by Ahlstrom, et al (1) and modified in part by Smith \& Schwartz (35). The technique assumes that this movement is a stochastic phenomenon and the magnitude is a function of a microscopic despersivity term. A similar technique was developed by others (27). An alternate technique by contrast uses spectral analysis assuming a vertical variability of hydraulic conductivity (17).

MODEL INPUT DEVELOPMENT

The methodology presented in this paper adopts the general premise that a soil stratum may be considered statistically homogeneous with the hydraulic conductivity having a lognormal PDF. A result of this premise is that the variation in the soil property may be represented by a curve similar to those presented in Figure 2. The upper curves illustrate PDF's of hydraulic conductivity for two different magnitudes of standard deviation but with the same mean value. These curves have lognormal PDF's. The lower curves illustrate the change in form of these PDF's when the $\log$ of hydraulic conductivity for these same two distributions are plotted. These curves have normal PDF's. It should be noted that a convenient aspect of the lognormal distribution is that negative values of the parameter are avoided.

The method selected to achieve the auto-correlation for the hydraulic conductivity values at adjacent nodes in the finite difference mesh is the stochastic nearest neighbor-model originally adapted by Smith (31) for use in groundwater flow applications. The method was utilized by 


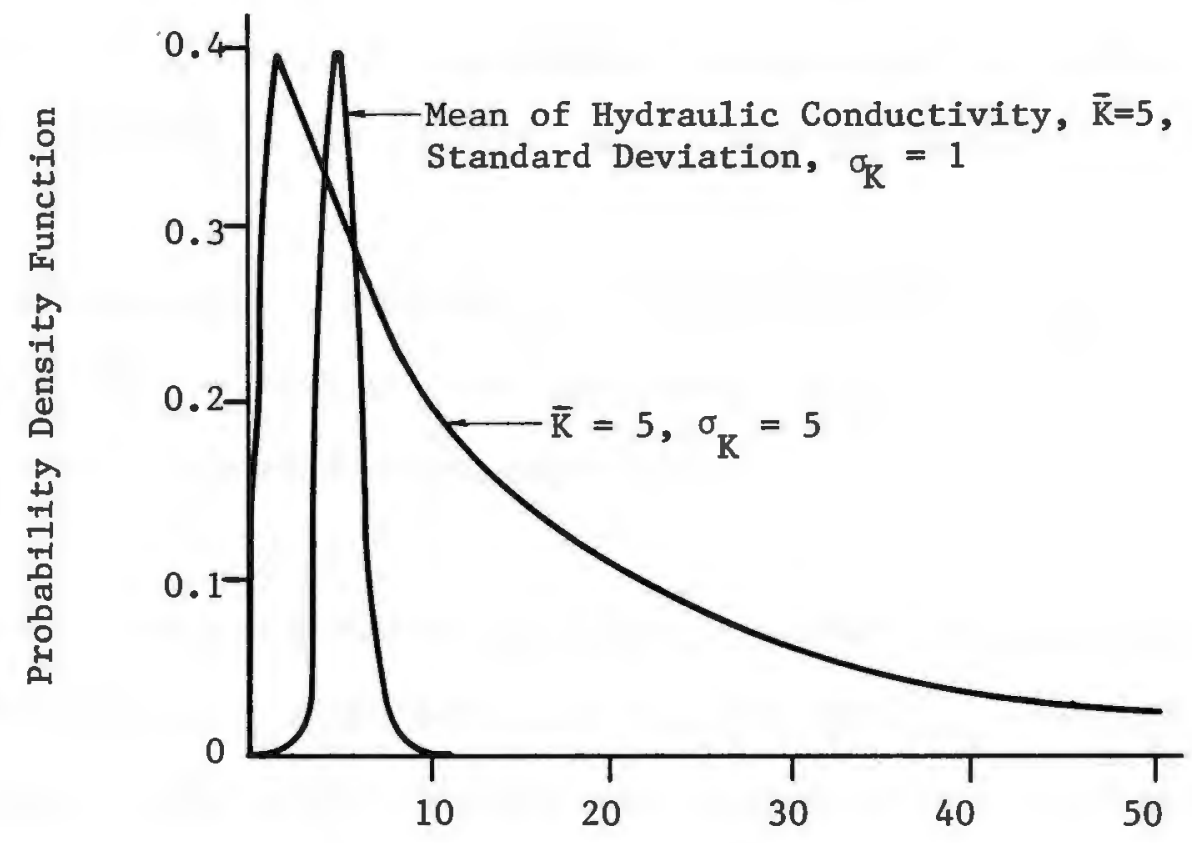

Hydraulic Conductivity, ft/day

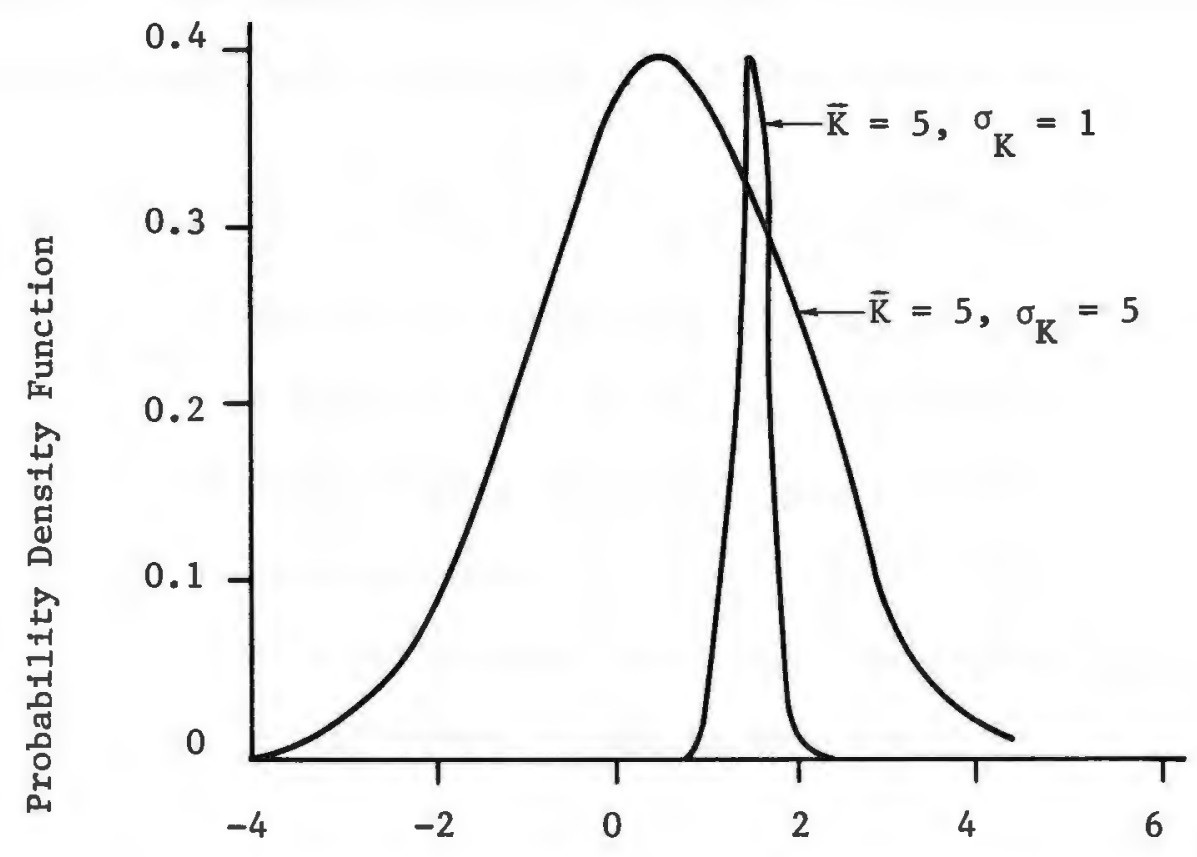

Ln of Hydraulic Conductivity, ft/day 
others in other fields $(5,7,9,25,42)$. A computer code was written to generate statistically independent realizations of hydraulic conductivity for each of the required Monte Carlo simulations.

Smith (31) presents a detailed development of the stochastic nearest neighbor model and much of this information is also available elsewhere $(32,33,34)$. A summary is presented here.

The finite difference method utilized to solve the groundwater flow problem requires as input hydraulic conductivities at regularly spaced grid points. These points or nodes are located at the center of a block of constant hydraulic conductivity, the so-called block centered approach. Figure 3 presents this diagramatically including the numbering system used. The nearest neighbor model uses the following equation to relate the conductivity at one point to all adjacent points.

$$
k_{i j}=\alpha_{x / 4}\left(K_{i, j-1}+k_{i, j+1}\right)+\alpha_{y / 4}\left(K_{i-1, j}+K_{i+1, j}\right)
$$

Where $\quad \mathbf{K}_{\mathbf{i j}} \quad$ = Hydraulic conductivity at nodal point, $i, j$.

i $=$ Row number with range from 1 to NROW

j $\quad=$ Column Number with range from 1 to NCOL

$\alpha_{x}, \alpha_{y}=$ Autoregressive parameters which indicate the degree of correlation of hydraulic conductivity in the $\mathrm{x}$ and y directions. Range is from 0 to 1.

This relationship may be put in matrix form and a stochastic element introduced as follows:

$$
\{K\}=[W]\{K\}+\{\varepsilon\}
$$




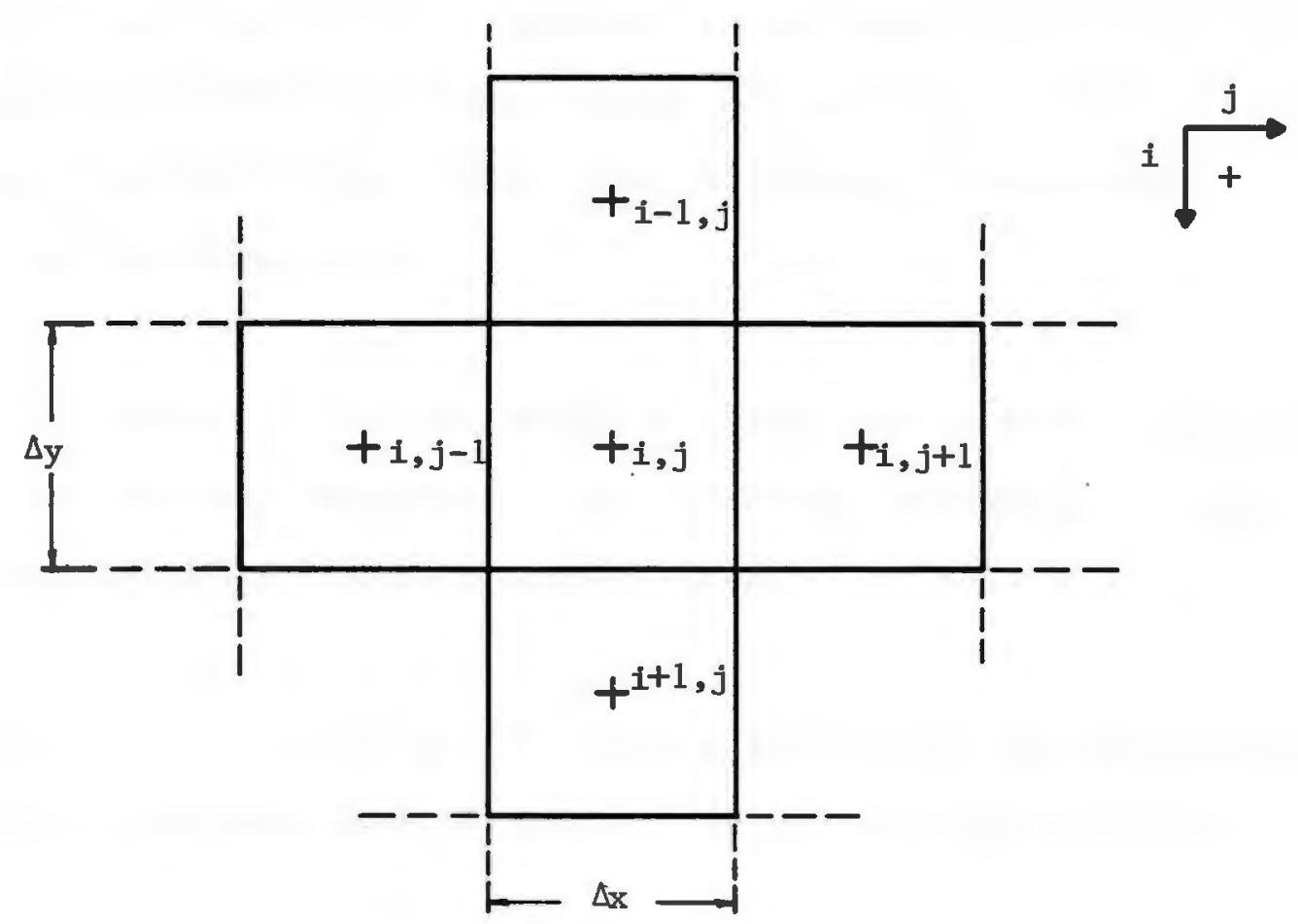

$$
\begin{gathered}
\mathrm{K}_{\mathrm{ij}}=\alpha_{\mathrm{x}} / 4\left(\mathrm{~K}_{i, j-1}+\mathrm{K}_{i, j+1}\right)+\alpha_{\mathrm{y}} / 4\left(\mathrm{~K}_{\mathrm{i}-1, j}+\mathrm{K}_{\mathrm{i}+1, j}\right) \\
- \text { Interior Node - }
\end{gathered}
$$

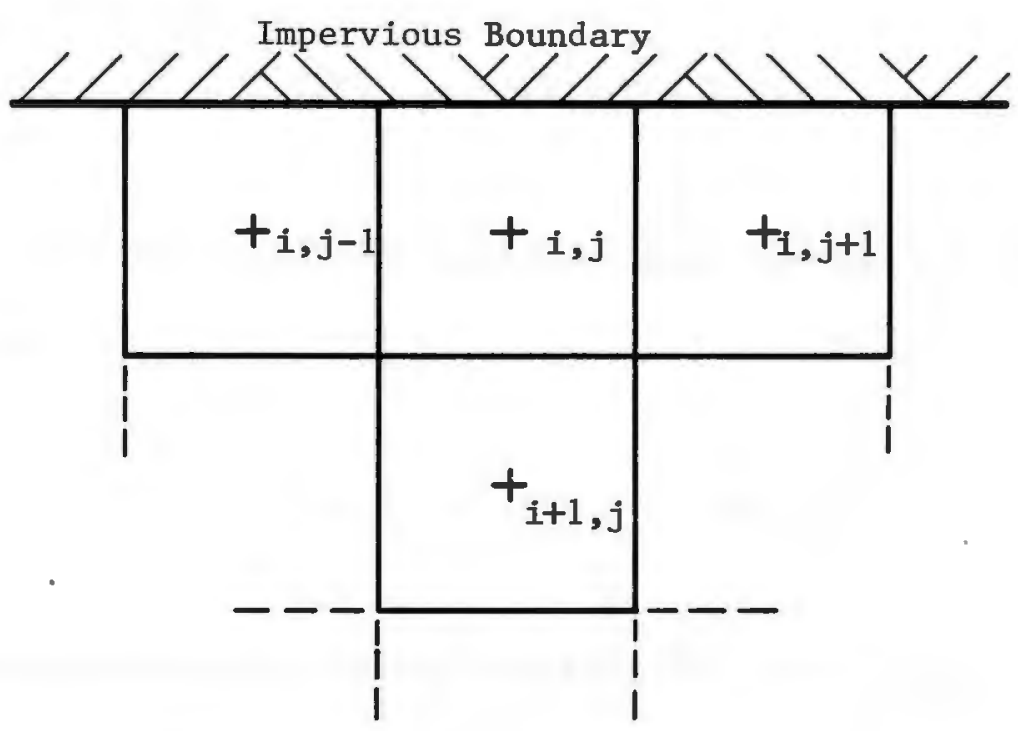

$$
\begin{gathered}
\mathrm{k}_{i, j}=\alpha_{x} / 3\left(\mathrm{~K}_{i, j-1}+\mathrm{K}_{i, j+1}\right)+\alpha_{y} / 3\left(\mathrm{k}_{i+1, j}\right) \\
- \text { Boundary Node - }
\end{gathered}
$$

Figure 3 - Correlation of Hydraulic Conductivity 
In this equation [W] is a weighting matrix consisting of NP rows and columns where NP is the total number of nodal points within the flow region, i.e., NP $=$ NROW $*$ NCOL. The [W] matrix is square while $\{\mathrm{K}\}$ and $\{\varepsilon\}$ are column matrices.

The $\{\varepsilon\}$ matrix is a column matrix of random numbers with a preselected mean and standard deviation. The basis for selecting the mean and standard deviation will be discussed later.

Since the $\{K\}$ matrix is the unknown result, we can rearrange this equation by bringing both $\{\mathrm{K}\}$ matrices to the left side as below.

$$
\{K\}-[W]\{K\}=\{\varepsilon\}
$$

We may then utilize the properties of an identity matrix and write

$$
([\mathrm{I}]-[\mathrm{W}])\{\mathrm{K}\}=\{\varepsilon\}
$$

Finally, we may solve for $\{$ K \}by multiplying by the inverse of ([I] - [W]) resulting in

$$
\{K\}=(I-W)^{-1}\{\varepsilon\}
$$

We now have the necessary matrix equation for this method. We need only develop the Weighting matrix [W], subtract it from the identity matrix, [I], compute an inverse, which may be called the Filter matrix, and multiply by the $\{\varepsilon\}$ matrix. 
It should be noted that the Filter matrix contains all the information concerning the autocorrelation of hydraulic conductivities for each block in the flow region. The $\{\varepsilon\}$ matrix contains the random component which is incorporated into the system of hydraulic conductivity values.

The nearest neighbor model is easily adapted for developing the [W] matrix for interior nodes. However, boundary nodes are another matter. Smith (31) assumed that the $\alpha$ term is divided by the number of adjacent nodes. Figure 3 also presents the situation along the upper boundary. Here this approach results in the following equation:

$$
k_{i j}=\alpha x / 3 k_{i, j-1}+k_{i, j+1}+\alpha y / 3 k_{i+1, j}
$$

Since a log normal distribution of hydraulic conductivity was adopted, the $\{\varepsilon\}$ matrix generated for each simulation will have a mean value of 0 , a standard deviation of 1 and a normal distribution. A transformation will later be made so that the hydraulic conductivity values will be $\log$ normally distributed. Smith (31) demonstrated that if the $\{\varepsilon\}$ matrix is multiplied by a constant term, $n$, any desired variance in the hydraulic conductivity values could be effected. He showed that this constant term, $n$, is a function of the desired variance of the conductivity distribution and the square root of a quantity $G$ defined as:

$$
\begin{aligned}
G= & \alpha_{y} \rho_{y}(2) / 8+\alpha_{x} \alpha_{y} \rho(1,1) / 2+\alpha_{y} \rho_{y}(1)-\alpha_{x} \rho_{x}(1)+\alpha_{y} / 8 \\
& +\alpha_{x}^{2} / 8+1+\alpha_{x} \rho_{x}(2) / 8
\end{aligned}
$$


where

$$
\begin{aligned}
& \rho_{x}(1), \rho_{y}(1) \quad \text { are the lag } 1 \text { correlation coefficients of } K \text { in } \\
& \text { the } x \text { and } y \text { directions } \\
& \rho_{x}(2), \rho_{y}(2) \quad \text { are the } 1 \mathrm{gg} 1 \text { correlation coefficients of } K \\
& \text { in the } x \text { and } y \text { directions } \\
& \rho(1,1) \quad \text { are the lag } 1 \text { in the } x \text { direction and lag } 1 \text { in } \\
& \text { the } y \text { direction correlation coefficients of } K
\end{aligned}
$$

The assumption of a $10 \mathrm{~g}$ normal distribution of hydraulic conductivity requires a transformation of the mean and standard deviation. It should be pointed out that the mean of the distribution of $1 n k$ does not equal the natural $\log$ of the mean of $\mathrm{K}$. Benjamin and Cornell (6) among others, present this transformation.

$$
\begin{aligned}
a_{\mathbf{y}}^{2} & = & \ln \left(\left(\sigma_{\bar{K}} / \overline{\mathbf{K}}\right)^{2}+1\right) \\
\bar{Y} & = & \ln (\bar{K}) \quad-\sigma_{\mathbf{Y}} / 2
\end{aligned}
$$

where:

$$
\begin{aligned}
\sigma_{\mathrm{y}}^{2} & =\text { variance of } \mathrm{Y} \\
\overline{\mathrm{Y}} & =\text { mean of } \mathrm{Y} \\
2 & =\text { variance of } K \\
\sigma_{\mathrm{K}} & =\text { mean of } K \\
\overline{\mathrm{K}} & =\ln \mathrm{K}
\end{aligned}
$$

This transformation is performed and the values $\sigma_{\mathrm{y}}$ and $\mathrm{Y}$ used with the $\{\varepsilon\}$ matrix. Following multiplication by the filter matrix and addition of the mean of the $\ln K$, a transformation back to $K$ is made. This transformation erodes some of the correlation although this has not been found to be significant (31). 
This method does not produce a distribution of hydraulic conductivity which exactly follows the desired correlation structure each time. This results from the randomness inserted by the random number generator, the exponential transformation, and boundary effects. However, each generated distribution is correlated and follows the desired correlation structure. When the ensemble of all Monte Carlo simulation is studied, a closer approximation to the desired correlation is obtained.

Smith (31) pointed out a problem at the boundary in maintaining the autocorrelation of the $K$ values. For this work it was decided to "shave off" the outer boundary nodes all around the block region. A $7 \times 7$ region, for example, would have the outer nodes deleted and then be transformed into a $5 \times 5$ region. This technique reduces the boundary effects but also reduces the size of the region which may be considered by this technique in light of the matrix inversion limitations.

This nearest neighbor method was chosen chiefly because it is easy to understand and, based on the work of previous researchers, it appears to represent the problem reasonably. Its major drawback is the amount of computer time needed to invert the weighting matrix, albeit this need only be done once for any region size and autocorrelation parameters and then stored. Indeed there are real limits to the size of the matrix that may realistically be inverted. This drawback effectively sets a limit on the number of nodes and hence influences mesh spacing and may influence the validity of results.

To alleviate this limitation, the flow region may be considered a compo- 
site of two or more "blocks" that contain statistically homogeneous and internally correlated hydraulic conductivity meshes. For example, the shaved 5 × 5 region may be systematically added to 3 other statistically equivalent regions resulting in a $10 \times 10$ region. The computer time to add these regions together is much less than that required for inverting the larger matrix.

The program utilized to solve the flow problem was developed by Reiter (29). This code was adapted from other available work for the two dimensional cross-sectional model (38).

MASS TRANSPORT SIMULATION

A computer code was developed to simulate transport across the hydraulic field resulting from a steady state solution. Transport results from hydraulic advection, a stochastic simulation of microscopic dispersion and dispersion resulting from variations in hydraulic conductivity. The major objective of this effort was to analyze the effects of hydraulic conductivity variations on dispersion.

Others $(17,35)$ have indicated that the variation in porosity is much less than the variation in hydraulic conductivity and probably does not significantly influence dispersion. The value of the porosity has therefore been assumed constant throughout the region.

The finite difference method assumes that the hydraulic head, hydraulic conductivity, porosity and therefore the resulting pore water velocity 
are constant within each individual block in the flow region. These velocities are calculated for each conductivity simulation and for each block within the region by the method suggested by Sauty (30).

Microscopic dispersion is incorporated into the model in a manner first described by Ahlstrom, et al (1) and then modified in part by Smith and Schwartz (35). In this method, dispersion is treated as a stochastic phenomenon. The water particle is assumed to undergo a random displacement in the direction of flow and another random displacement perpendicular to flow. These displacements are calculated from the following two equations :

$$
\begin{aligned}
& \mathrm{DL}=(24 \mathrm{DLC} * \mathrm{DELT})^{\frac{1}{2}} *(0.5-\text { RANOL }) \\
& \mathrm{DT}=(24 \mathrm{DTC} * \mathrm{DELT})^{\frac{1}{2}} *(0.5-\text { RANOT })
\end{aligned}
$$

In these equations, DL and DT are the displacements in the longitudinal and transverse directions, respectively, relative to the direction of advective transport. DLC and DTC are the longitudinal and transverse dispersion coefficients. RANOL and RANOT are random numbers normally distributed with a mean of zero and a standard deviation of one then divided by six to keep the range of values in the -0.5 to +0.5 area.

Kelly (21) developed a direct relationship, which has been adopted for this study, between grain size and microscopic dispersivity. This relationship may be expressed as: 


$$
\hat{0}=0.08 \mathrm{D}_{50}
$$

Where

and $\delta$ = dispersivity, in meters,

$$
\mathrm{D}_{50}
$$

= mean particle size in millimeters

The longitudinal dispersion coefficient, DLC, is taken equal to the dispersivity times the velocity, $V_{L}$, in the direction of flow. The transverse dispersion coefficient, $D F C$, is taken equal to a percentage of the dispersivity, DSPRAT, times the dispersivity and velocity, $\mathrm{V}_{\mathrm{L}}$. The expressions from Ahlstrom, et al (1) for microscopic dispersion are then modified by these assumptions and units adjusted to obtain the following :

$$
\begin{aligned}
& \mathrm{DL}=\left(6.3 * \mathrm{D}_{50} * \mathrm{~V}_{\mathrm{L}} * \mathrm{DELT}\right) * \text { RANOL } \\
& \mathrm{DT}\left(6.3 * \mathrm{D}_{50} * \mathrm{~V}_{\mathrm{L}} * \mathrm{DELT}\right) * \text { DSPRAT } * \text { RANOT }
\end{aligned}
$$

Since the advective transport direction will not always coincide with the $X$ and $Y$ axes the displacements in the $X$ and $Y$ directions are obtained by a transformation.

The computer code simulates water movement from the upgradient side of the region to the downgradient side. A particle is released from the upgradient boundary and moves across the region as dictated by the 
advective displacements and stochastic dispersive displacements. When an impervious boundary is encountered, the particle is reflected back into the region. Eventually the water particle encounters the downgradient boundary. The location, travel time and other data are recorded and available for statistical analysis.

Monte Carlo simulations result in a great deal of output. It was necessary to develop a large data collection and analysis system to deal with the data. Data from this study was stored in disk data files as it was produced and saved for use and analysis later. The Statistical Analysis System (SAS) has been used in the analysis of the data.

The data output to disk files was varied but consists of information on the hydraulic conductivity means and correlation for each simulation, travel times and dispersion, hydraulic head values both mean values and those for selected rows from each simulation. These data are used as input to the SAS programs. SAS programs have been written to perform all types of statistical calculations and checks, as well as to plot results.

RESULTS AND DISCUSSION

A considerable amount of computer simulation was performed during this study. One major limitation immediately encountered was the inordinate amount of computer time required to invert the ( $I-W)$ matrix. The size of the matrix increases very rapidly with the region size since a new row and column is added for each new finite difference block in the 
region. The results of inverting this matrix for three region sizes, 7 by 7,12 by 12 and 12 by 22 suggest an exponential relationship between the total number of nodes in the region and the required computer time.

The fact that the computer time required to perform the inversion increases so rapidly with an increase in region size effectively sets a practical limit on the region size which could be considered in one block. The largest region considered in this study was a 12 by 22 region which required that a 264 × 264 matrix be inverted. We shall see later that some techniques may be utilized to somewhat relax this limitation.

The arithmetic, geometric and harmonic means of all the simulations of hydraulic conductivity for the numerous Monte Carlo solutions were computed. These mean values were compared to an effective hydraulic conductivity, $K_{q}$, calculated on the basis of the flow quantity by the following equation:

$$
\mathrm{K}_{\mathrm{q}}=\frac{\mathrm{Q}}{\mathrm{h}_{\mathrm{L}}} \frac{\mathrm{L} * \dot{S}}{\mathrm{H}}=\frac{\mathrm{Q} \mathbf{S}}{\mathrm{h}_{\mathrm{L}}}
$$

where $Q$ is the flow quantity, $h_{L}$ is the total head loss within the region, $I$ is the total length over which that head loss occurs, $H$ is the height of the flow region and $\dot{S}$ is a shape factor computed in accordance with a technique suggested by Warren and Price (40) which will be presented later.

The value of $\mathrm{S}$ is a shape factor which may be directly compared with the 
shape factor, $N_{D} / N_{F}$, in a flow net solution or the form factor in the method of fragments. $N_{D}$ and $N_{F}$ are the number of equipotential drops and flow lines repectively in the flow net solution. The results of similar Monte Carlo simulations of flow regions represented by random and uncorrelated hydraulic conductivity meshes have indicated that the geometric mean is a good estimate of the effective hydraulic conductivity $(8,18,40)$.

A second measure of the effective hydraulic conductivity in terms of mean travel time was computed for each Monte Carlo simulation. This effective hydraulic conductivity on the basis of travel time, $K_{t}$, was calculated by making assumptions similar to those used in calculating $\mathrm{K}_{\mathrm{q}}$. Figure 4 presents a typical layout of a flow region with approximately horizontal uniform flow from left to right. The flow is not precisely uniform due to the dispersion present in the flow simulation.

The velocity of flow, v, may be calculated by the following formula:

$$
v=\frac{K^{i}}{\bar{n}}=\frac{K h_{L}}{\bar{n}} \frac{L_{L}}{L}
$$

The mean velocity of all flow particles traversing the flow region, $\bar{v}$, may be calculated by dividing the length of the particle travel, $\mathrm{I}_{\mathrm{T}}$, by the mean travel time, $\bar{t}$, as below:

$$
\bar{v}=L_{T} / \bar{t}
$$

The effective hydraulic conductivity on the basis of travel time, $K_{t}$, has been calculated by setting $v$ equal to $\bar{v}$ in the above two equa- 


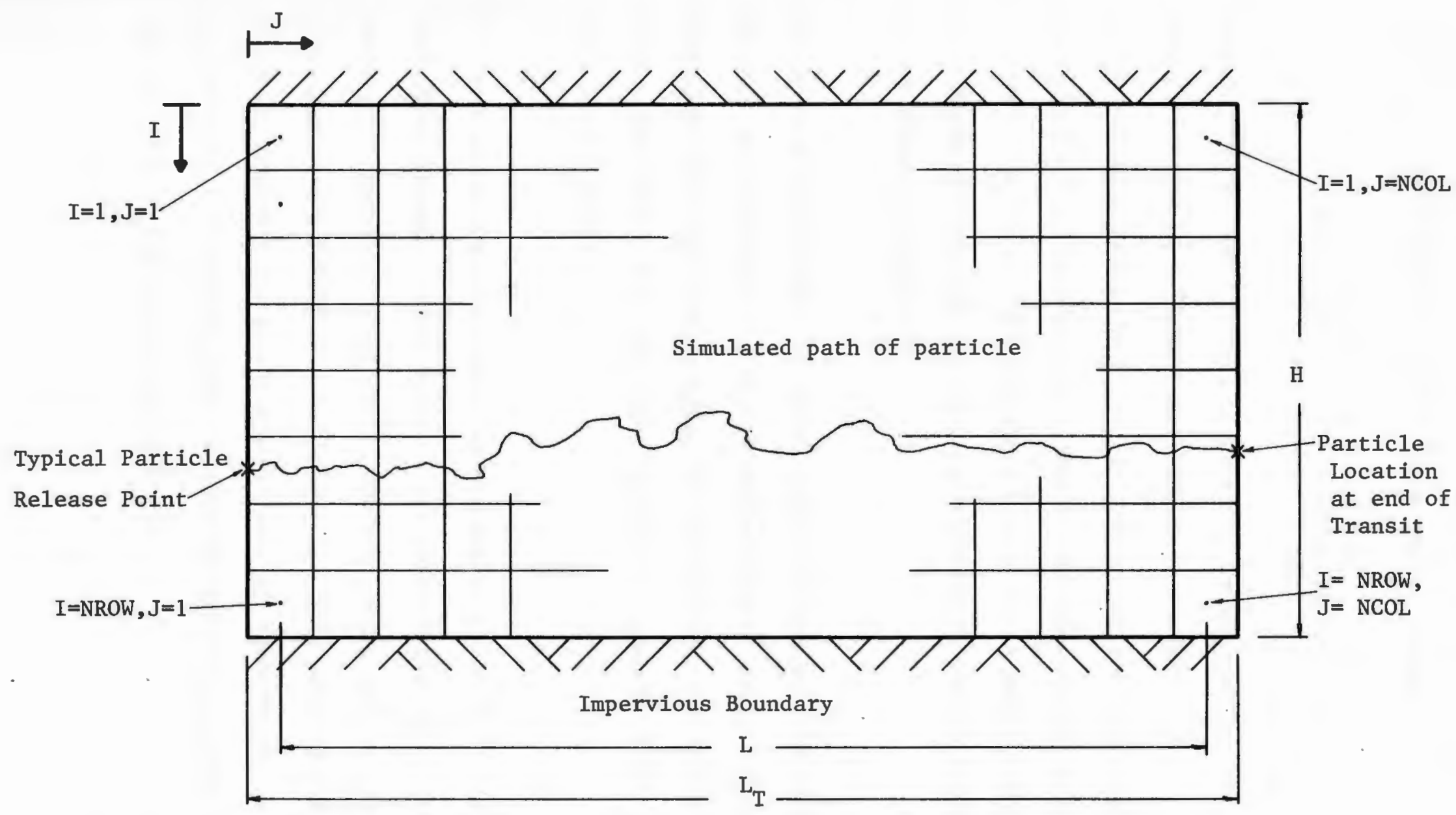

Along left boundary where $\mathrm{J}=1$, hydraulic head is maintained constant at value of $\mathrm{H}$ Along right boundary where $\mathrm{J}=\mathrm{NCOL}$, hydraulic head is maintained constant at value ${ }^{u}$ of $H_{L}$ Mean head loss in flow region $=h_{L}=H_{u}-H_{L}$ 
tions, introducing the height of the region by inserting in both the numerator and denominator and solving for $\mathrm{K}_{t}$ resulting in the following:

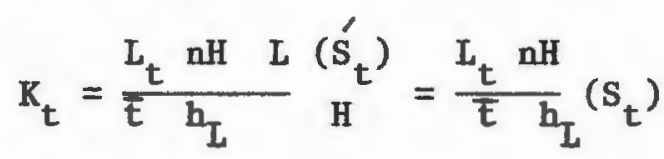

where $\mathrm{L}_{T}$ is the total length of particle travel and for the uniform flow case will equal $\mathrm{L}, \mathrm{n}$ is the porosity, $t$ is the mean travel time and $\mathrm{L}, \mathrm{H}$ and $h_{L}$ are as defined above. $S_{t}$ is a shape factor dependent on boundary conditions and is computed in a manner similar to 's and will be discussed later. $S_{t}$ is a modified shape factor which may be compared to the other shape factors, $S$, and the shape factor in the flow net solution or by the method of fragments.

Twenty-five variations of flow region size and characteristics were studied to determine the relationship between these calculated means. Generally for each flow region, 40 Monte Carlo characterizations were done. The means and effective hydraulic conductivities for each of these were computed.

In addition to calculation of mean values for each of the Monte Carlo characterizations, a mean value of all. Monte Carlo characterizations was computed. Correlation of the geometric mean, and the two effective hydraulic conductivity values (flow quantity and travel time) were visually observed by plotting and also by computation of correlation coefficients. A typical plot of these results is presented in Figure 5 and the results are summarized in Table 1 . 

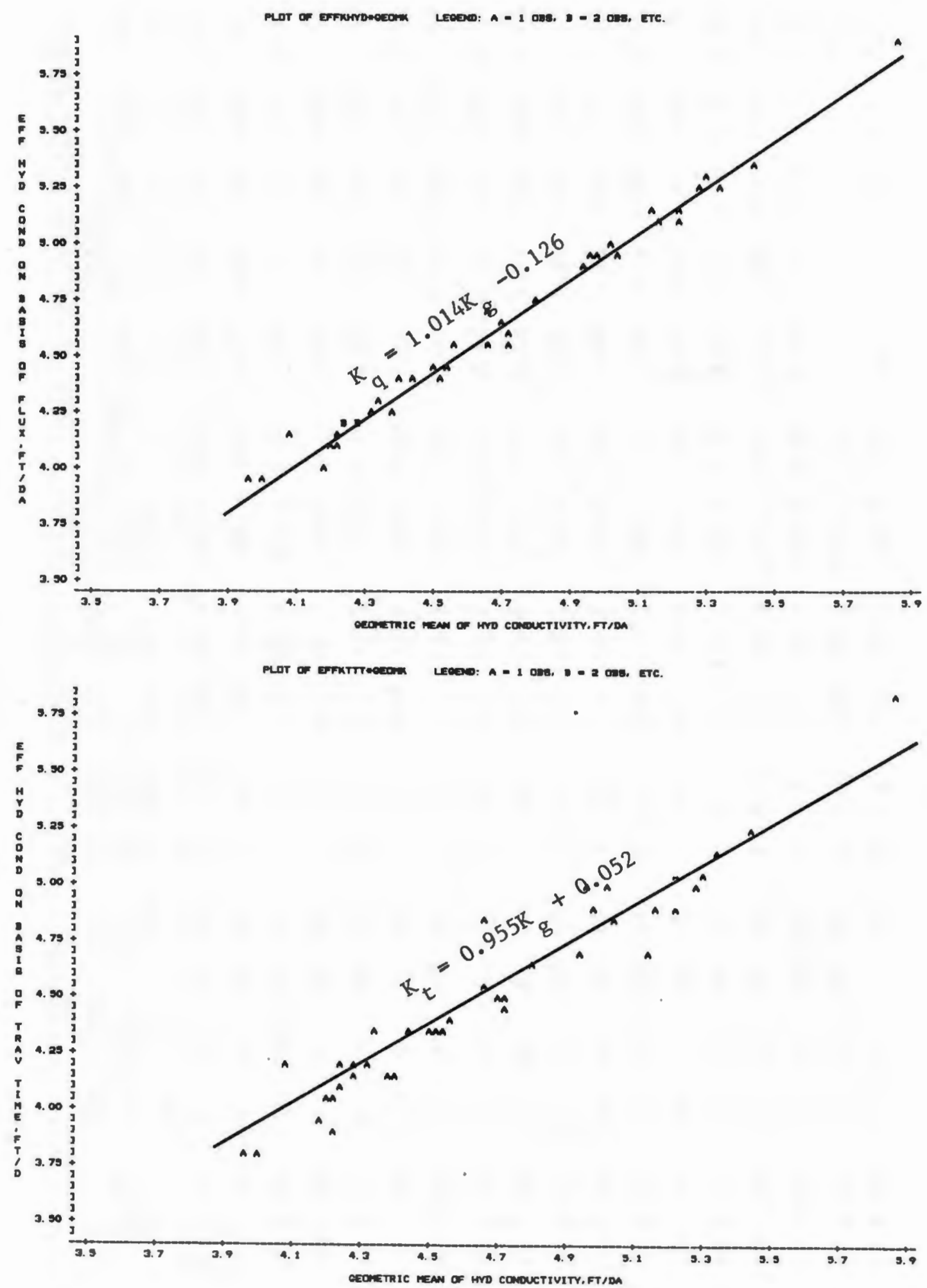

Figure 5 - Correlation of Effective Hydraulic Conductivity with Geometric Mean 
TABLE 1 - SUMMARY OF FLOW SIMULATIONS

\begin{tabular}{|c|c|c|c|c|c|c|c|c|c|c|c|c|c|c|c|c|c|}
\hline \multirow{3}{*}{ File } & \multirow{3}{*}{$\begin{array}{l}\text { Region } \\
\text { Size }\end{array}$} & \multirow{3}{*}{$\begin{array}{l}\text { Block } \\
\text { Size }\end{array}$} & \multirow{2}{*}{\multicolumn{3}{|c|}{$\begin{array}{l}\text { Auto. Corr. } \\
\text { Data }\end{array}$}} & \multirow{3}{*}{$\begin{array}{l}\text { No. } \\
\text { of } \\
\text { Sims }\end{array}$} & \multirow{3}{*}{$\begin{array}{l}\text { No. } \\
\text { of } \\
\text { Wa- } \\
\text { ves }\end{array}$} & \multirow{3}{*}{$\begin{array}{l}\text { Time } \\
\text { Int., } \\
\text { Days }\end{array}$} & \multirow{3}{*}{$\begin{array}{l}\text { Kol- } \\
\text { mog } \\
\text { D } \\
\text { Stat }\end{array}$} & \multirow{3}{*}{$\begin{array}{l}\text { Y } \\
\text { Coor. } \\
\text { at } \\
\text { End }\end{array}$} & \multirow{3}{*}{$\begin{array}{l}\text { Total } \\
\text { Travel } \\
\text { Time, } \\
\text { Days }\end{array}$} & \multicolumn{6}{|c|}{$\begin{array}{c}\text { Means of Hydraulic } \\
\mathrm{ft} / \mathrm{day}\end{array}$} \\
\hline & & & & & & & & & & & & $\overline{\text { Arith }}$ & Geom & Harm & Flux & T.Tim. & S.Dev \\
\hline & & & $\mathbf{x}$ & $y$ & & & & & & & & & $\mathbf{K}$ & $\mathrm{K}_{\mathrm{h}}$ & & 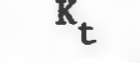 & $\mathbf{K}$ \\
\hline 1 & $10 \times 20$ & $10 \times 10$ & .8 & .8 & .68 & 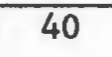 & 1 & 1 & .032 & 0 & $1 / 2$ & 0.0 & 4.63 & 4.20 & 4.53 & .44 & .10 \\
\hline 2 & $10 \times 20$ & $10 \times 20$ & .8 & .4 & .68 & . & 1 & 1.6 & .115 & 60.4 & 168.5 & 4.84 & 4.62 & 4.40 & 4.59 & 4.51 & 1.54 \\
\hline 3 & $10 \times 20$ & $10 \times 20$ & $.8 \mathrm{~L}$ & $.8 \mathrm{~L}$ & .68 & 40 & 1 & 1.6 & .15 & 59.5 & 171.1 & 5.01 & 4.65 & 4.32 & 4.57 & 4.44 & 2.01 \\
\hline 4 & $10 \times 10$ & $10 \times 10$ & .0 & .8 & .68 & 40 & 1 & 0.8 & .01 & 59.7 & 39.8 & 5.13 & 4.77 & 4.45 & 4.75 & 4.61 & 1.99 \\
\hline 5 & $5 \times 5$ & $5 \times 5$ & .8 & .8 & .68 & 40 & 2 & 6.4 & .01 & 35.0 & 9.5 & 5.13 & 4.78 & 4.46 & 4.80 & 5.28 & 1.90 \\
\hline 6 & $10 \times 20$ & $10 \times 20$ & .8 & .8 & .68 & 10 & 4 & 1.6 & .024 & 58.4 & 176.6 & 4.86 & 4.51 & 4.19 & 4.42 & 4.30 & 1.95 \\
\hline 7 & $10 \times 20$ & $10 \times 20$ & . & .8 & .68 & . & 3 & 2.0 & - & 58.6 & 234.3 & 5.05 & 4.69 & 4.36 & 4.63 & 4.54 & 1.99 \\
\hline 8 & $10 \times 20$ & $10 \times 20$ & .8 & .4 & 1.0 & 40 & 1 & 1.6 & .01 & 60.0 & 173.0 & 5.09 & 4.60 & 4.15 & 4.54 & 4.39 & 2.40 \\
\hline 9 & $10 \times 20$ & $10 \times 20$ & .8 & .8 & .68 & . & 1 & 1.6 & .01 & 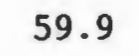 & 169.7 & 5.04 & 4.68 & 4.34 & 4.62 & 4.52 & 2.02 \\
\hline 10 & $10 \times 40$ & $10 \times 20$ & .8 & .8 & .68 & 40 & 1 & 3.2 & .01 & 59.6 & 703.4 & 4.99 & 4.62 & 4.28 & 4.53 & 4.45 & 2.02 \\
\hline 2 & $10 \times 40$ & $10 \times 20$ & .0 & .8 & .68 & (10 & 1 & 0.0 & .15 & 00.2 & 4 & 5.00 & 4.68 & 4.32 & 4.59 & 4.51 & 2.08 \\
\hline 12 & $10 \times 40$ & $10 \times 20$ & .8 & .8 & .68 & 40 & 1 & 6.4 & .014 & 59.2 & 702.2 & 5.02 & 4.64 & 4.29 & 4.54 & 4.46 & 2.08 \\
\hline 1 & $10 \times 40$ & $10 \times 10$ & .8 & .8 & .68 & , & 1 & 3.2 & .04 & 60.8 & 697.0 & 3.02 & 4.64 & 4.29 & 4.55 & 4.48 & 2.08 \\
\hline 14 & $20 \times 40$ & $10 \times 10$ & .8 & .8 & .68 & 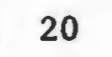 & - & 3.2 & .15 & 111.7 & 700.0 & 4.99 & 4.61 & 4.27 & 4.54 & 4.46 & 2.09 \\
\hline 15 & $20 \times 40$ & $10 \times 20$ & .8 & .8 & .68 & 20 & 1 & 3.2 & .15 & 111.2 & 699.7 & 4.98 & 4.62 & 4.28 & 4.55 & 4.46 & 2.03 \\
\hline 16 & $10 \times 60$ & $10 \times 20$ & .8 & .8 & .68 & o & 1 & 4.8 & .15 & 59.5 & 1576.2 & 5.06 & 4.67 & 4.31 & 4.57 & 4.49 & 2.11 \\
\hline 17 & $10 \times 60$ & $10 \times 10$ & .8 & .8 & .68 & 40 & 1 & 4.8 & .052 & 59.5 & 1576.7 & 5.06 & - & - & - & - & 2.14 \\
\hline 18 & $10 \times 20$ & $10 \times 20$ & 1.0 & 1.0 & - & 1 & 40 & 1.6 & .15 & 60.2 & 151.9 & 5.0 & 5.0 & 5.0 & 5.0 & 5.0 & 0.0 \\
\hline
\end{tabular}


TABLE 1 - SUMMARY OF FLOW SIMULATIONS (CONTINUED)

\begin{tabular}{|c|c|c|c|c|c|c|c|c|c|c|c|c|c|c|c|c|c|c|}
\hline \multirow{3}{*}{ File } & \multirow{3}{*}{$\begin{array}{l}\text { Region } \\
\text { Size }\end{array}$} & \multirow{3}{*}{\multicolumn{2}{|c|}{$\begin{array}{l}\text { Block } \\
\text { Size }\end{array}$}} & \multirow{2}{*}{\multicolumn{3}{|c|}{$\begin{array}{l}\text { Auto. Corr. } \\
\text { Data }\end{array}$}} & \multirow{3}{*}{$\begin{array}{l}\text { No. } \\
\text { of } \\
\text { Sims }\end{array}$} & \multirow{3}{*}{$\begin{array}{l}\text { No. } \\
\text { of } \\
\text { Wa- } \\
\text { ves }\end{array}$} & \multirow{3}{*}{$\begin{array}{l}\text { Time } \\
\text { Int., } \\
\text { Days }\end{array}$} & \multirow{3}{*}{$\begin{array}{l}\text { Kol- } \\
\text { mog } \\
\text { D } \\
\text { Stat }\end{array}$} & \multirow{3}{*}{$\begin{array}{l}\text { Y } \\
\text { Coor. } \\
\text { at } \\
\text { End }\end{array}$} & \multirow{3}{*}{$\begin{array}{l}\text { Total } \\
\text { Travel } \\
\text { Time, } \\
\text { Days }\end{array}$} & \multicolumn{6}{|c|}{$\begin{array}{r}\text { Means of Hydraulic } \\
\mathrm{ft} / \mathrm{day}\end{array}$} \\
\hline & & & & & & & & & & & & & & & Harm & Flux & & S.Dev \\
\hline & & & & $x$ & y & & & & & & & & & & $a_{1}$ & & & $n$ \\
\hline 19 & $10 \times 20$ & 10 & $\times 20$ & .8 & .2 & .0 & 40 & 1 & 1.6 & & 60.7 & (2) & 5.11 & 4.65 & 4.23 & 4.62 & 4.47 & 2.35 \\
\hline 20 & $10 \times 20$ & 10 & $\times 20$ & .8 & .2 & 0.9 & 40 & 1 & 1.6 & .15 & 60. & 169.8 & 5.005 & 4.64 & 4.3 & 4.6 & 4.7 & 2.04 \\
\hline 21 & $10 \times 60$ & 0 & $\times 20$ & .8 & .8 & .68 & 40 & 1 & 1.0 & .048 & 60.3 & 314.5 & 4.96 & 4.58 & 4.23 & 4.48 & 4.41 & 2.05 \\
\hline 22 & $10 \times 60$ & 10 & X 20 & .8 & .8 & .68 & 40 & 1 & .44 & .066 & 60. & 143.0 & 5.07 & 4.68 & 4.33 & 4.59 & 4.50 & 2.07 \\
\hline 23 & $10 \times 20$ & 10 & $\times 20$ & .8 & .8 & .68 & 40 & 1 & 1.6 & - & 98.4 & 228.0 & 5.12 & 4.72 & 4.36 & 4.62 & 4.50 & .12 \\
\hline 24 & $10 \times 20$ & 10 & X 20 & 0 & 0 & - & 1 & 32 & 16 & - & 88 & 211.8 & 50 & 5 & 5 & 50 & 5 & 0.0 \\
\hline 25 & $10 \times 20$ & 10 & $\times 20$ & .0 & 1.0 & - & 1 & 40 & 1.6 & - & 98.8 & 200.5 & 5.0 & 5.0 & 5.0 & 5.0 & 5.0 & 0.0 \\
\hline 2 & $10 \times 20$ & 0 & $\times 20$ & 0 & 0.4 & .84 & 40 & 1 & 1.0 & & 60. & 173.7 & 4 & 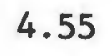 & 4. & 4.51 & (3) & .98 \\
\hline 27 & $10 \times 20$ & 10 & $\times 20$ & .8 & 0.8 & .68 & 40 & 2 & 1.6 & .01 & 58.8 & 254.91 & 5.01 & 3.54 & 2.51 & 3.32 & 3.06 & 4.786 \\
\hline 28 & $10 \times 20$ & 10 & X 20 & .0 & .8 & .68 & 40 & 1 & 1.6 & & 60.0 & .11 & 5.01 & 4.98 & 4.96 & 4.98 & 4.97 & 0.50 \\
\hline 29 & $20 \times 40$ & 10 & X 20 & .8 & .8 & .68 & 20 & 1 & 3.2 & & 109.6 & 2058.4 & 2.99 & - & - & - & .52 & 2.47 \\
\hline 30 & $10 \times 20$ & 10 & X 20 & .4 & .3 & .95 & 40 & 1 & 1.6 & .15 & 59.8 & 170.5 & 5.01 & 4.66 & 4.34 & 4.58 & 4.47 & 1.97 \\
\hline
\end{tabular}

Note: Uniform flow in all files except 7 and 24 which simulate S Flow and 23 and 25 with cutoff. Values listed for $K_{q}$ and $K_{t}$ have been adjusted by use of the shape factor $S$. 
The results of these computations support the observation that the geometric mean is the best measure of the effective hydraulic conductivity in terms of flow quantity and travel time. The effective hydraulic conductivity calculated both by flow quantity and travel time are relatively close with the flow quantity value generally somewhat larger. These results generally agree with Butler and Gundlach (10) who determined that the equivalent area for flow and travel time were not always equal. Their flow regions were largely one dimensional.

Two boundary flow configurations were considered in addition to uniform flow, i.e., flow from the left boundary to the right boundary of a rectangular region. The two flow configurations are presented in Figure 6. The first flow configuration is described as $S$ flow and consists of flow from the upper three nodes of the left boundary to the lower three nodes of the right boundary. This case could simulate a general two dimensional flow case such as around an obstruction or low hydraulic conductivity occulusion. The second flow configuration is described as cutoff and consists of flow from the left upper boundary to the bottom right boundary. This case is intended to simulate flow beneath a dam by representing one half of the flow region.

Warren and Price (40) have indicated that the geometric mean of the hydraulic conductivity is a good approximation of the effective hydraulic conductivity even for non-uniform flow provided a shape factor is utilized to adjust the value for the shape of the flow region. They suggest that the magnitude of this shape factor, $S^{\prime}$ in equation 15 , be determined by first calculating the effective hydraulic conductivity 

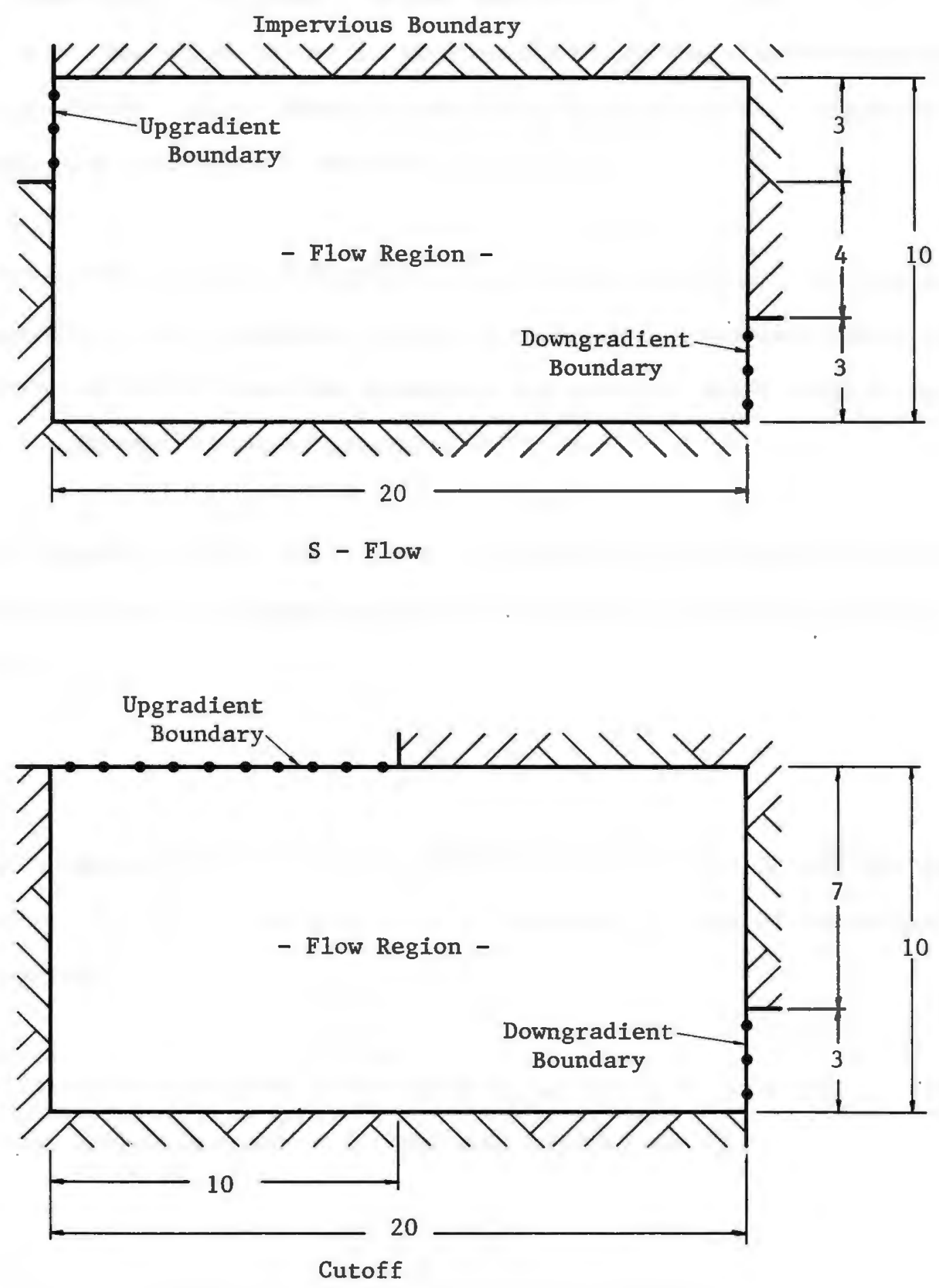

Figure 6 - Alternate Boundary Conditions 
of the flow region using a singular known value of hydraulic conductivity. This effective value of hydraulic conductivity should then be divided into the singular known value and the result is the shape factor. The shape factor is then multiplied by the effective hydraulic conductivity before comparing with the geometric mean. Examples of these computations are presented elsewhere (2).

The results of this study tend to support this technique. For the cases considered, the adjusted values of effective hydraulic conductivity generally fall between the geometric and harmonic means and is closest to the geometric mean as presented in Table 1.

The importance of this finding is that the mean flow rate may be estimated by rearranging equation 15 to solve for the flow rate, $Q$, as below:

$$
\mathrm{Q}=\frac{\mathrm{K} \mathrm{h}_{\mathrm{L}}}{\mathrm{S}}
$$

The geometric mean should be used for the value of $K$ and the shape factor, S, may be determined by a flow net solution or the method of fragments.

A similar rearrangement of equation 18 and taking $\mathrm{L}_{\mathrm{T}}$ equal to $\mathrm{L}$, results in the following equation for the mean travel time, $\bar{t}$ :

$$
\overline{\mathrm{t}}=\frac{\mathrm{L} \cdot \mathrm{n} \cdot \mathrm{H} \cdot \mathrm{S}_{\mathrm{t}}}{\mathrm{K} \mathrm{h}_{\mathrm{L}}}
$$

The mean travel time may then be approximated by using the geometric 
mean of hydraulic conductivity for $K$ and the shape factor from a flow net solution or the method of fragments. Although this technique has not been theoretically proven and for nonuniform flow $\mathrm{L}_{\mathrm{T}}$ may be unknown exactly and may not equal $\mathrm{L}$, the results developed here suggest that this technique can provide reasonable estimates of mean travel time.

The stochastic nearest neighbor process model was utilized to generate random meshes of hydraulic conductivity which are autocorrelated. Each of the individual representations within the ensemble of representations making up each Monte Carlo simulation was analyzed to determine the magnitude of correlation by row and column. The formulation below was utilized to calculate this value and may be found in many references, e.g. (32):

$$
\rho(L)=\frac{\frac{1}{n}-\frac{1}{i=1}\left(K_{i}-\bar{K}\right) *\left(K_{i+L}-\bar{K}\right)}{(n-1) \sum_{i=1}^{n}\left(K_{i}-\bar{K}\right)^{2}}
$$

In this equation $\rho(L)$ is the autocorrelation coefficient for the lag $L$. $\mathrm{L}$ is a whole number with the range zero to one minus the number of elements in the row or column considered. $K$ is the value of hydraulic conductivity and $K$ is the mean value. The denominator is the variance of the hydraulic conductivity. It may be easily shown that if I equals zero, then $\rho(0)$ equals 1.0 .

A graph of a typical result of this computation for lags from one to three is presented in Figure 7. The concept of integral scale as dis- 


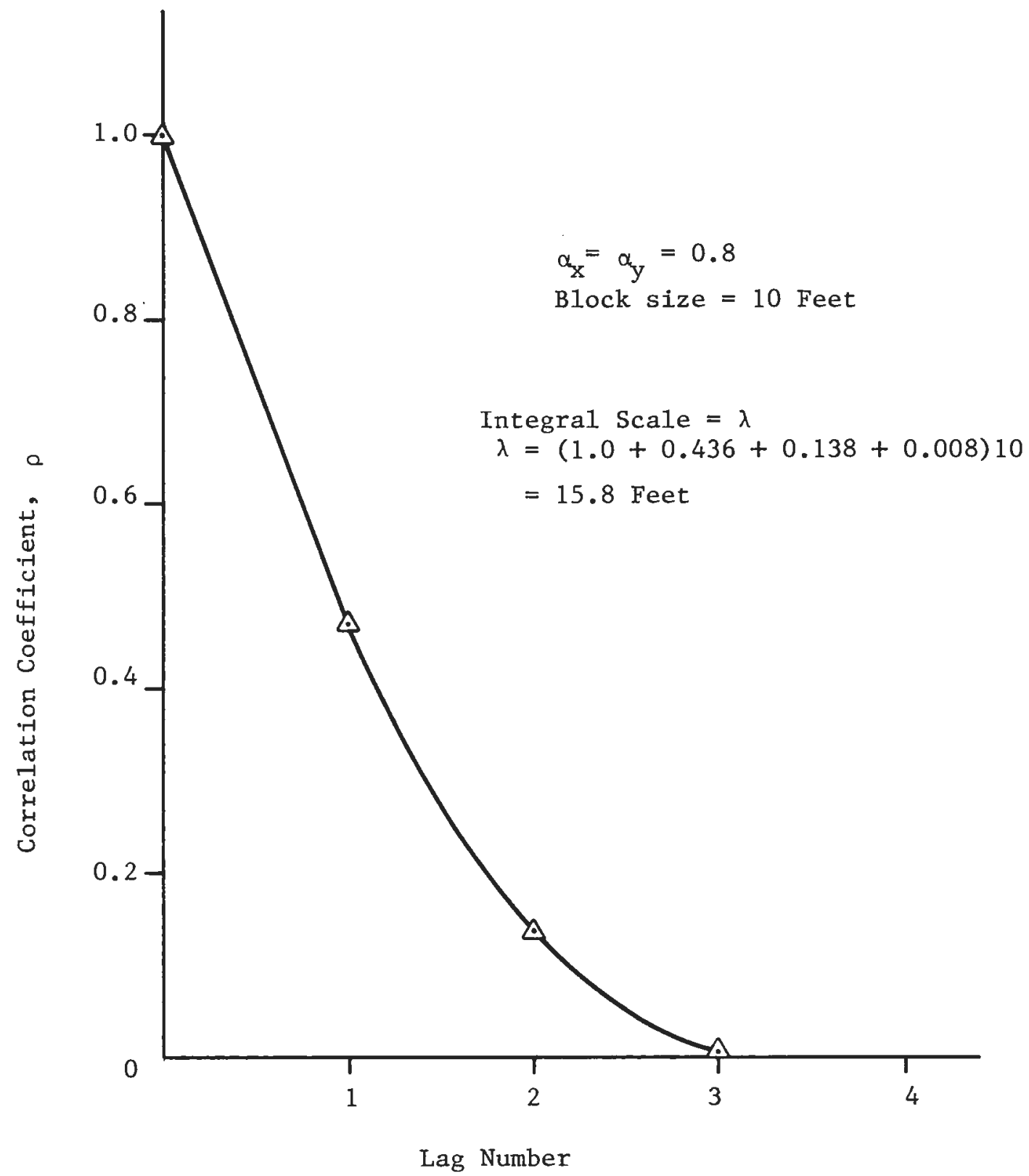

Figure 7 - Correlation Coefficient Versus Lag Number 
cussed by Smith and Freeze (33) is utilized herein to conceptualize the degree of dependency of the hydraulic conductivity value at one node with the value at adjacent nodes. The integral scale has been estimated as the summation of the positive auto-correlation coefficients times the block size. As indicated, the integral scale, $\lambda$, equals 15.8 for the data in Figure 7.

Smith (31) recognized a problem with the autocorrelation resulting from boundary effects. This problem was mitigated somewhat by deleting the boundary blocks of the region after development. Aldinger (2) demonstrates the effects on the autocorrelation coefficients of deleting these boundary blocks. Also presented are the effects on these same autocorrelation coefficients caused by the log transformation to hydraulic conductivity. The results indicate a slight loss of correlation when the boundaries blocks were deleted and some additional loss when the log transformation was completed. This total loss was slight, particularly for the integral scale.

The effect of the region size on the integral scale is demonstrated in Figure 8. It is clear that as the region size increases, the integral scale increases, although at a decreasing rate. This figure also demonstrates the effects of stacking blocks of internally autocorrelated hydraulic conductivity meshes to model larger regions without utilizing a larger FILTR matrix. There is a loss in the integral scale although this loss is relatively small even when the region is increased by three times. The figure also demonstrates that as the region size increases, the integral scale continues to increase even with the same FILTR 


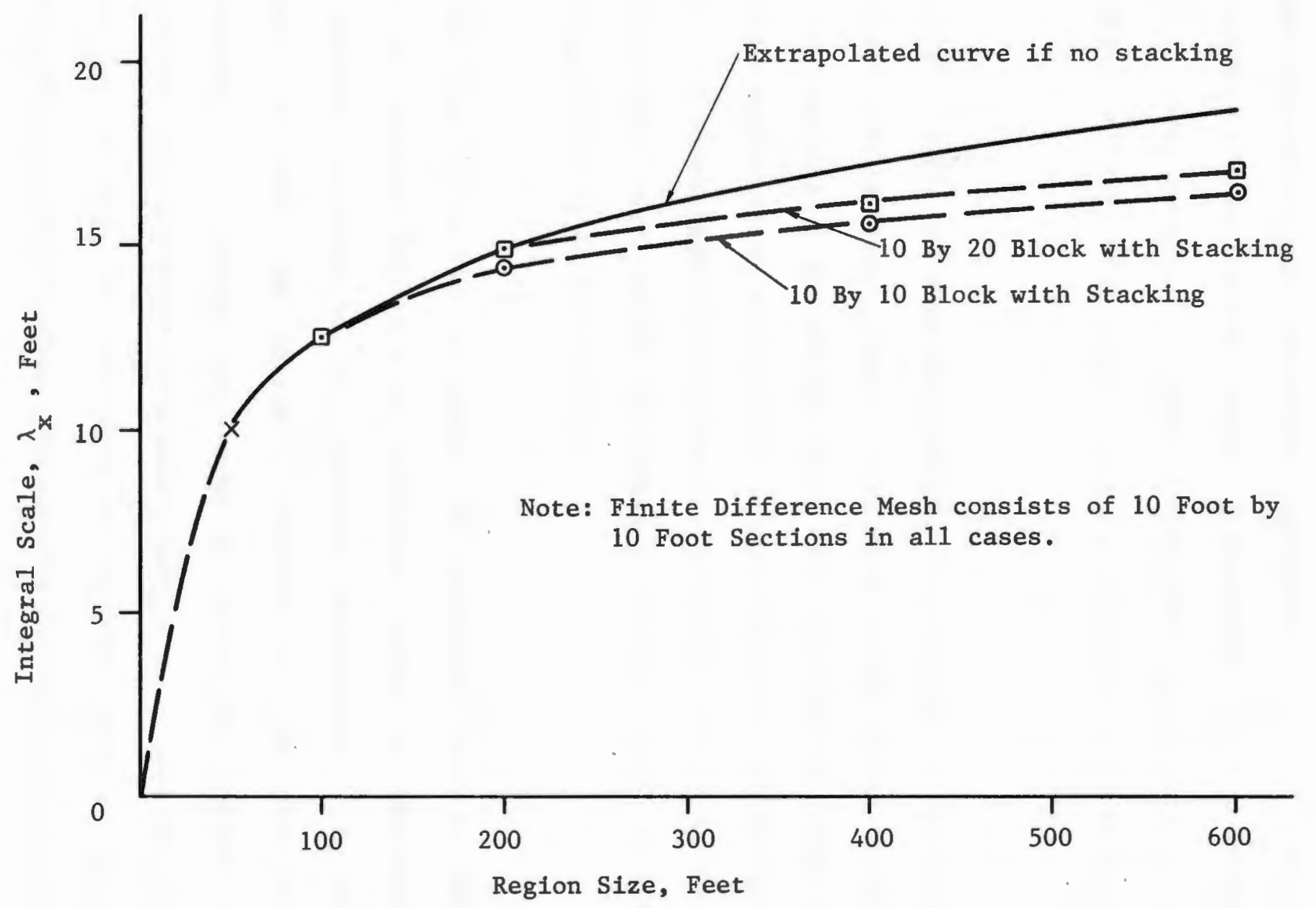

Figure 8 - Integral Scale Versus Region Size 
matrix.

Sensitivity of Ensemble Means and Variances to the Number of Simulations

Computer computations are relatively inexpensive. However, the practicing engineer does not want to do more computations than are necessary to obtain reliable answers. A study of the number of simulations required to compute reliable mean values of various parameters was then conducted.

Initially consider the mean and standard deviation of the hydraulic conductivity formulations. Figure 9 presents a plot of an accumulating mean of the lag 3 arithmetic mean and standard deviation of the hydraulic conductivity as each new equiprobable representation of the Monte Carlo simulations is calculated. This plot demonstrates that the cumulative mean very rapidly approaches a constant value, in this case after approximately 8 simulations.

The SAS program written to compute the cumulative mean of the lag 3 means and standard deviation is an inexact estimate of the actual mean and standard deviation of all preceding observations. The procedure computes the mean and standard deviation of the previous three observations. A running mean value of these two results is then computed as each new mean and standard deviation is computed for a new observation and the two left from the earlier group of three after deleting the first observation. The results have been found accurate to approximately 3 to 4 decimal places for the mean value. However, the 


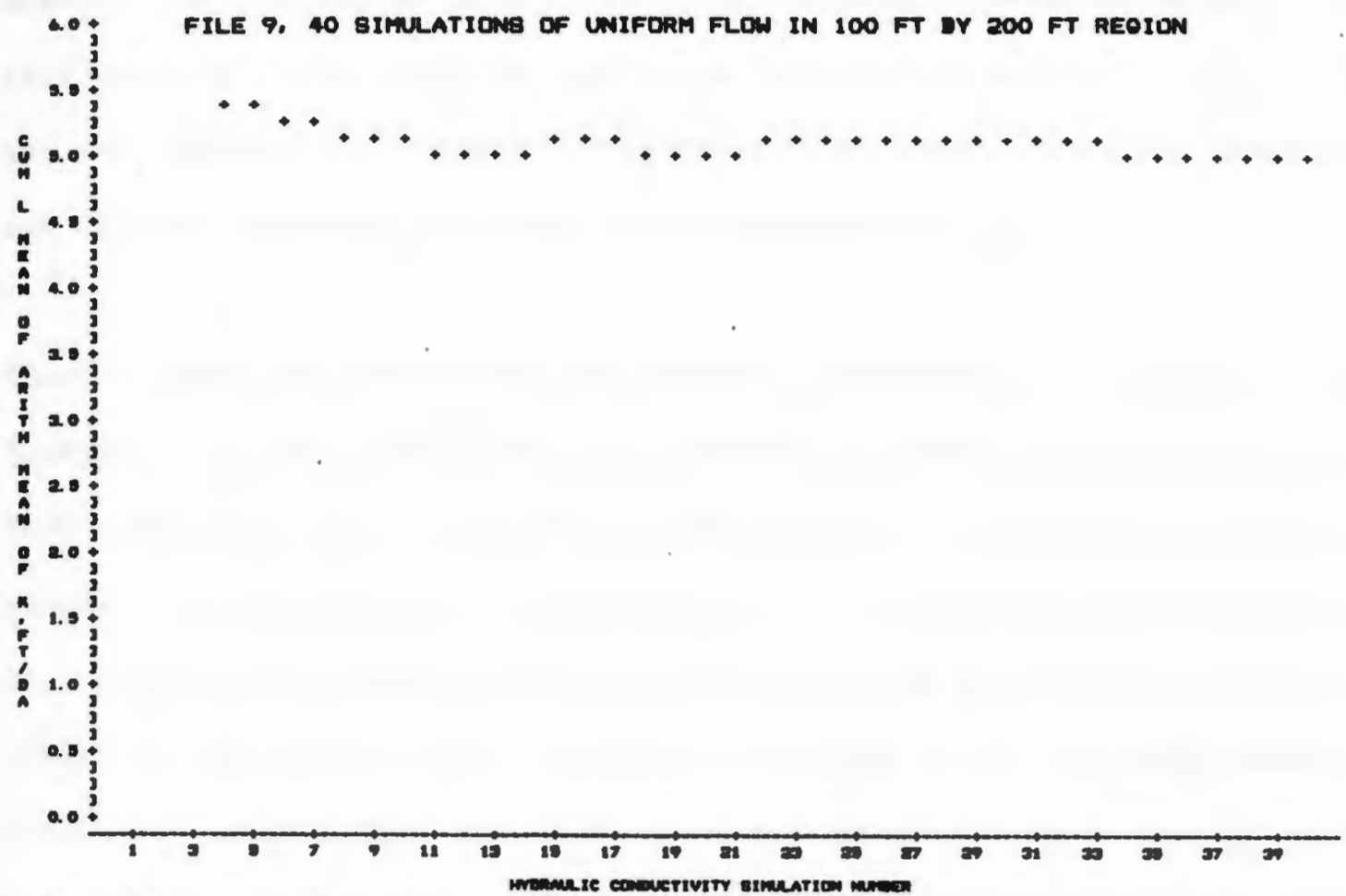

nure: $\quad 3000$ kn mresing valus

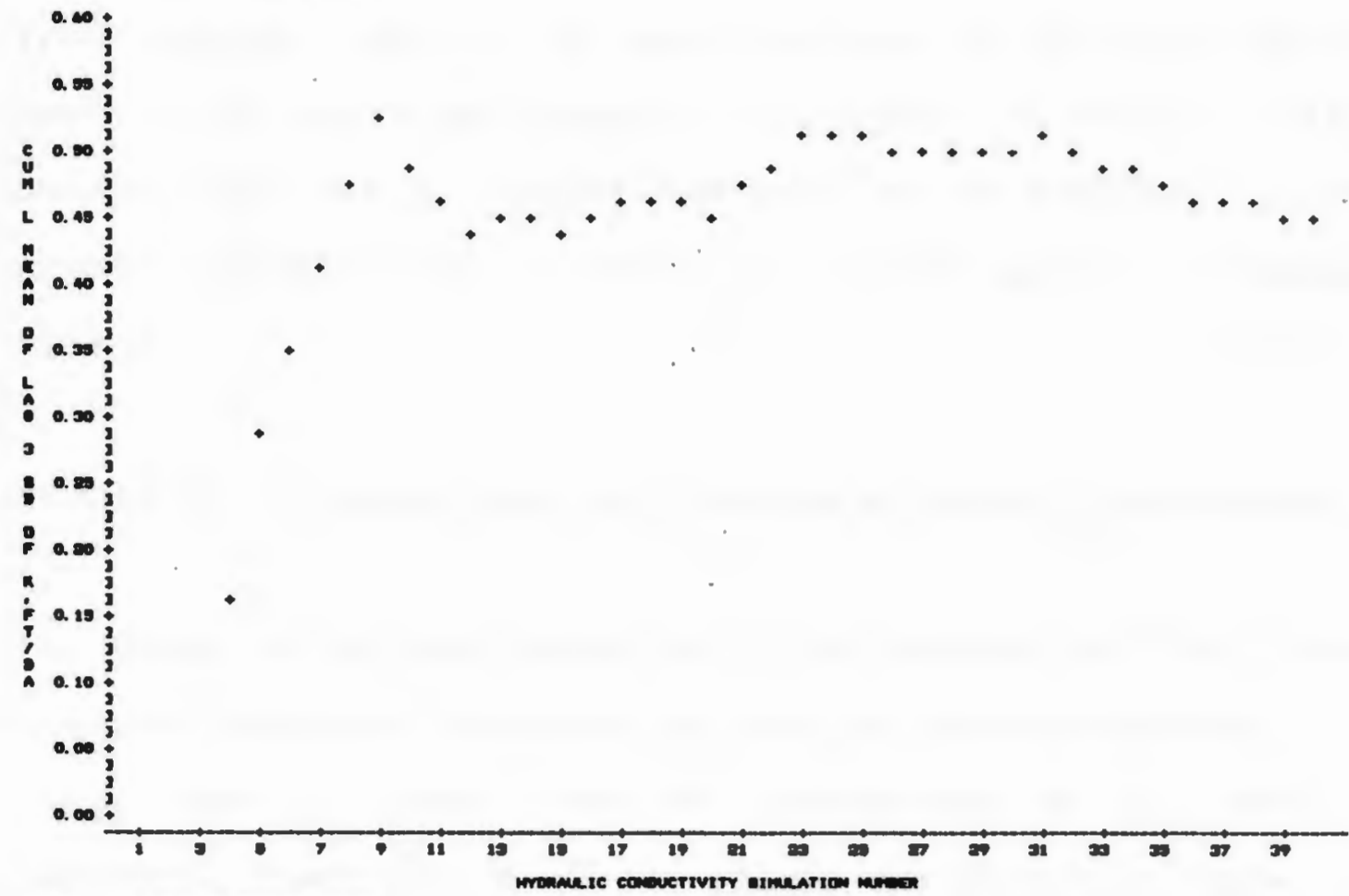

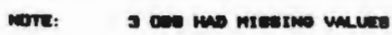

Figure 9 - Cummulative Mean Values of Mean and Standard Deviation of Hydraulic Conductivity Versus Simulation Number 
mean of the cumulative mean of the lag 3 standard deviation computed by this technique may only be used as a qualitative measure of the trend and its stability. A precise measure of the actual standard deviation can only be computed after the entire ensemble is sampled.

Similar plots for the effective hydraulic conductivity in terms of flow quantity, $K_{q}$, and travel time, $K_{t}$, respectively have been developed (2). These indicate that a fairly constant value is rapidly approached, usually in less than 10 simulations. This probably results from the fact these are a measure of the variation of the mean hydraulic conductivity in the flow region. The model attempts to set the mean value of hydraulic conductivity equal to the same value for each simulation so that these related mean values should also approach consistent values fairly rapidly. However, the cumulative means of the lag 3 standard deviation for these terms fluctuates considerably. In Figure 9, a truly constant value is not achieved even after the 40 simulations in this ensemble although after the first 20, the fluctuations are greatly reduced.

Sensitivity of Ensemble Means and Variances to Number of Water Particles

The number of particles which need to be released and their travel simulated to allow an estimate of the mean and standard deviation of the travel time is another important consideration in the amount of computation time required. A SAS program was written to compute the cumulative mean of the lag 3 mean and standard deviation of the travel time for particle transit. A typical plot for one run is presented in 
Figure 10. This figure illustrates that a mean value of travel time is reached after approximately 20 water particles have transited the region. The cumulative mean of the lag 3 standard deviation of the travel time fluctuates 20 or 40 percent after 20 water particles, reaching a fairly consistent, although still slightly decreasing value, after approximately 80 water particles.

Fried (15) gives an expression for the longitudinal dispersion coefficient, $D_{L}$, assuming that the development of particle concentration at some distant point is normally distributed. He illustrates that this coefficient is directly related to the variance of the travel times. The equation developed for a long travel distance relative to the dispersion is:

$$
D_{L}=\frac{\bar{U}^{2}\left(2 \sigma_{t}\right)^{2}}{8 \bar{t}}
$$

where $\bar{U}$ and $\bar{t}$ are the mean velocities and travel time, respectively, and $\sigma_{t}$ is the standard deviation of the travel time.

The distance, $x$, over which the dispersion occurs equals the product $\mathrm{Ut}_{0.5}$ and may be substituted in this equation resulting in the following equation:

$$
D_{L}=\frac{\sigma_{t}^{2} x^{2}}{{ }^{2}(\bar{t})} 3
$$



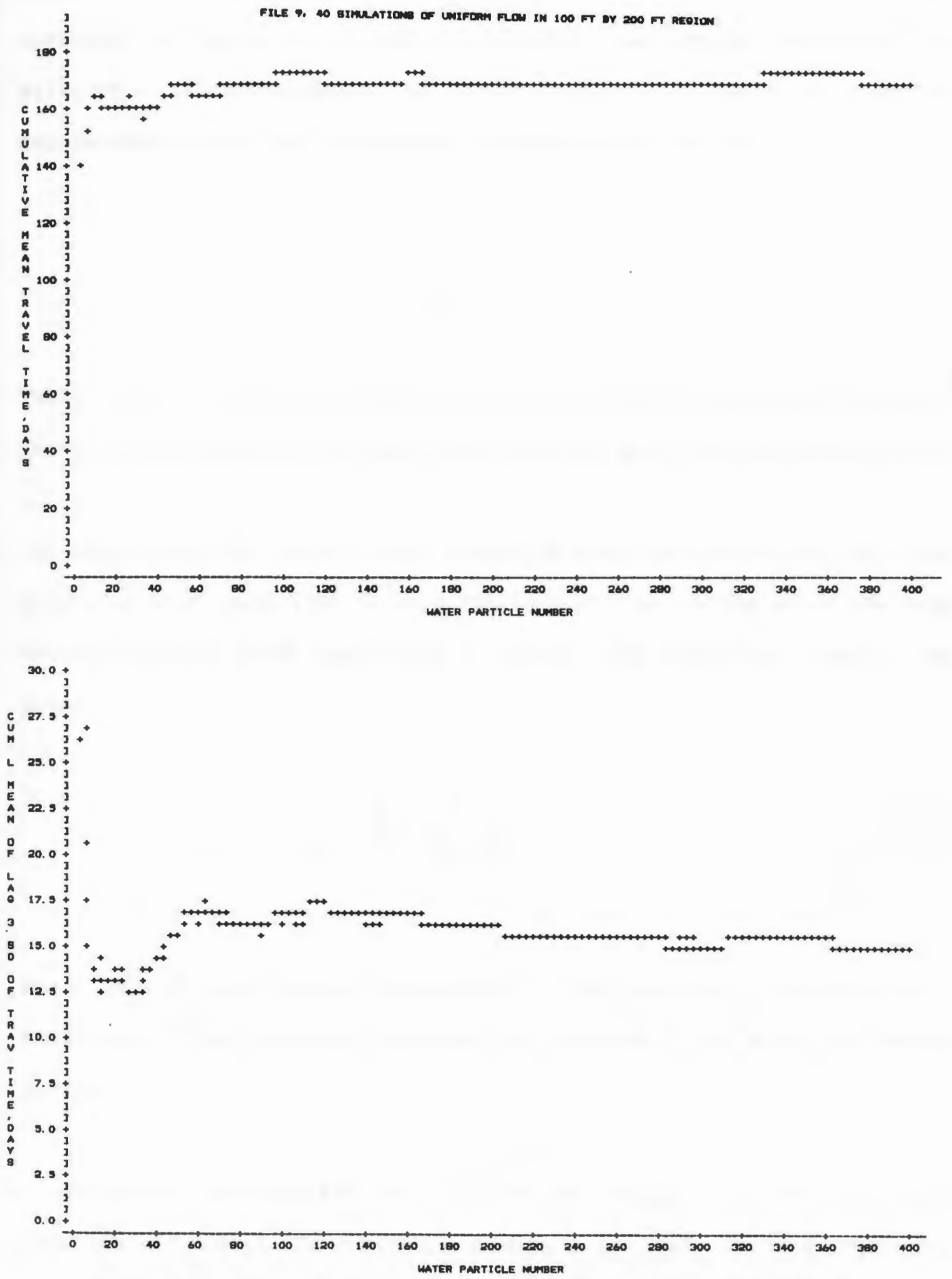

Figure 10 - Cumulative Mean Values of the Mean and Standard Deviation of Travel Times Versus Particle Number 
These expressions illustrate the relationship between the standard deviation of the travel time, $\sigma_{t}$, and the longitudinal dispersion coefficient. The macroscopic or field scale dispersivity, $\delta_{f}$, may be determined by dividing by the mean velocity to arrive at:

$$
\delta_{f}=\frac{\sigma t^{x}}{2 t^{2}}
$$

These results and others indicate that the 400 water particles simulated is sufficient to compute a mean and standard deviation for travel time.

The macroscopic or field scale lateral dispersion coefficient, $D_{T}$, has been similarly shown (15) to be a function of the scatter about the mean ending location after traversing a region. The following equation was given:

$$
\mathrm{D}_{\mathrm{T}}=\frac{1}{2} \frac{\sigma \mathrm{y}^{2} \overline{\mathrm{U}}}{\mathrm{x}}
$$

where $\sigma_{y}$ is the standard deviation of the normalized location perpendicular to the primary direction of flow and $\bar{U}$ and $x$ are as defined earlier.

A record was maintained of the starting and ending $y$ coordinate of each particle. A normalized ending y coordinate was then computed along with a cumulative mean and cumulative mean standard deviation as each new 
record of water transport was added. The same was computed for the $y$ coordinate at the estimated time to transit half the region. A typical plot of these results is presented in Figure 11. These results also indicate that the 400 water particles are sufficient to reach a consistent mean value for these variables.

\section{Normality Check on Travel Times}

The ensemble of travel times was utilized to plot a breakthrough curve. Figure 12 presents a representative sample of a breakthrough curve. These curves are similar to a concentration versus time curve and their similarity of shape supports the premise of general conformance of normality.

A check for the goodness of fit of the assumption of a normal distribution for the ensemble of travel time records was made. A SAS program was written to test the null hypothesis that the data could be a random sample from a normal distribution. A Kolomogorov D statistic was computed and compared with critical values. A Kolomogorov statistic was utilized rather than the more common chi square check since it is more easily adapted to continuous uncategorized data.

Table 1 presents the results of this check for several flow ensembles. Several of the normality checks support the assumption of a normal distribution with a general tendency to do so as the transit distance increases. Cases 10,11 and 12 suggest that as the time interval decreases, there is a greater likelihood of acceptance of a normal dis- 
FILE 9. 40 SIMLATIONE OF UNIFORM MLON IN 100 FT IV 200 FT REOTON

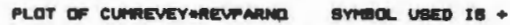
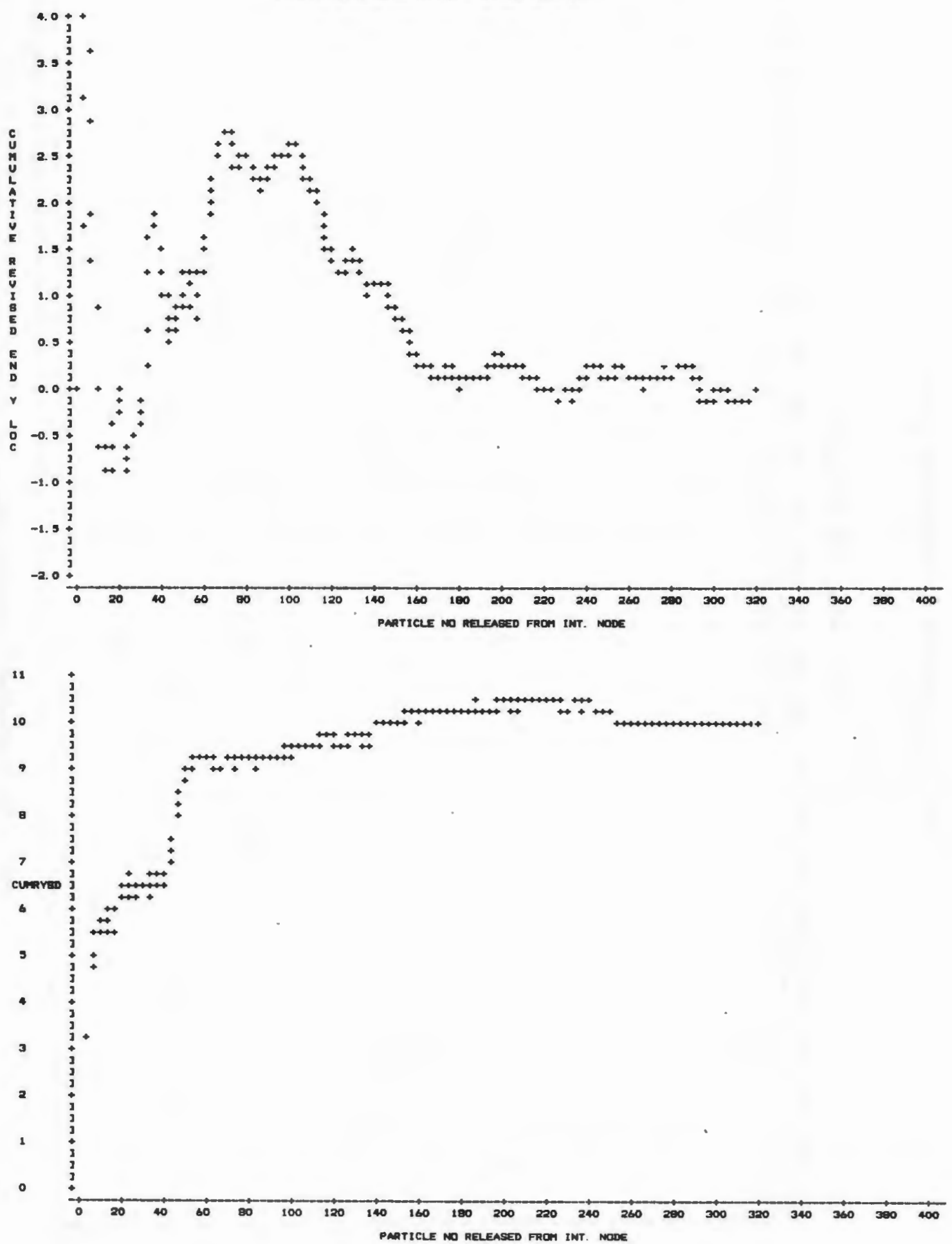

Figure 11 - Cummulative Mean of Mean and Standard Deviation of Ending $Y$ Location Versus Particle Number 
FILE 9, 40 BIMULATIONB OF UNIFQRM FLOU IN 100 FT UY 200 FT REOION

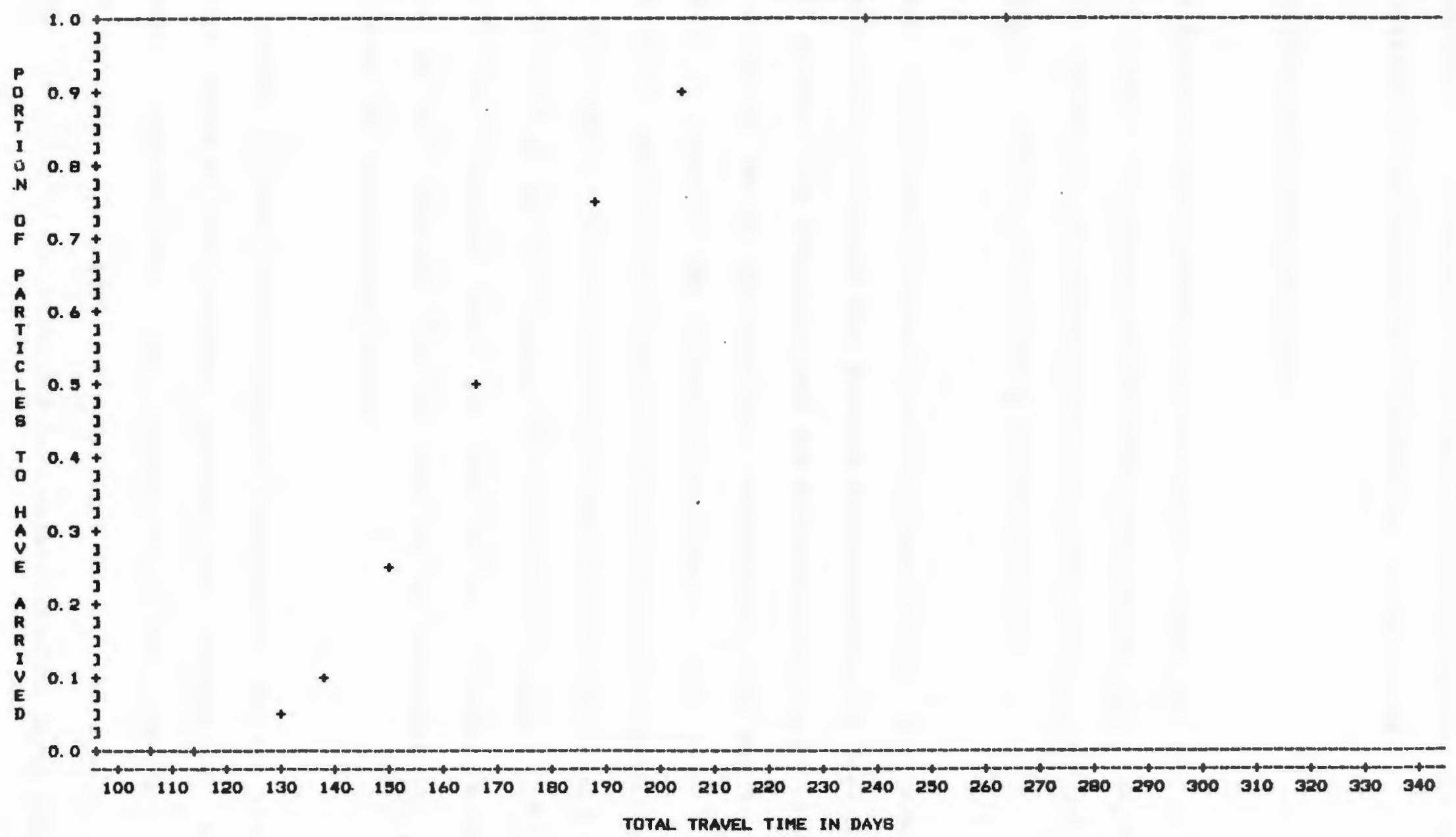

Figure 12 - Typical Breakthrough Curve 
tribution. A Kolomogorov D statistic of 0.10 or greater was considered acceptance of the assumption of normality for this study.

Hydraulic Head Value Sensitivity

The hydraulic head value along two rows was tabulated for each of the simulations. This tabulation was then available to allow computation of mean values and variations resulting from changes in region size, hydraulic gradient, conductivity and other factors.

Figure 13 presents typical results from one of the Monte Carlo simulations. The upper plot presents the mean hydraulic head versus the node number. The averaging over the 40 Monte Carlo simulations results in a fairly smooth straight line relationship. The middle curve in Figure 13 presents the standard deviation and the lower curve the variance of the hydraulic head for the 40 Monte Carlo simulations versus the node number. These plots clearly demonstrate the boundary effects. At the ends of the flow region the head is held constant and therefore the standard deviation is zero at these nodes. At nodes further away, there is less influence from the boundary and therefore the standard deviation and variance are greater.

The number of Monte Carlo simulations required to reach a fairly consistent value of the hydraulic head was also investigated. Figure 14 presents a typical plot of the results of one such investigation at an interior node. This plot indicates that the initial simulation had a value very close to the resulting mean value and after three simulations 

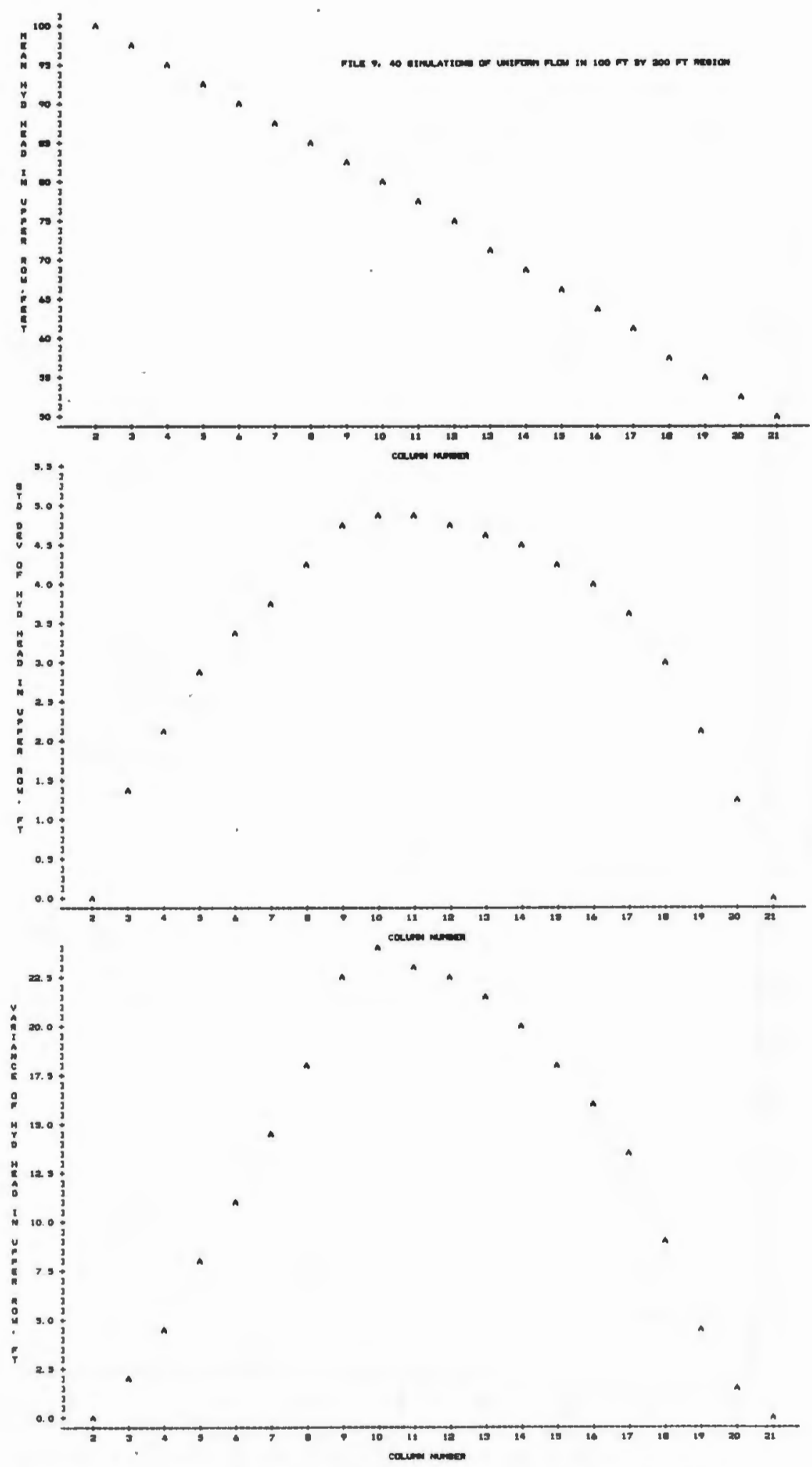

Figure 13 - Mean, Standard Deviation and Variance of Hydraulic Head Versus Node Number 
FILE 27, 40 BIMULATIONS OF UNIFORM FLOW IN 100 FT BY 200 FT REOION

PLOT OF CUMHIIMNEBIMNO BYMBDL USED IB +

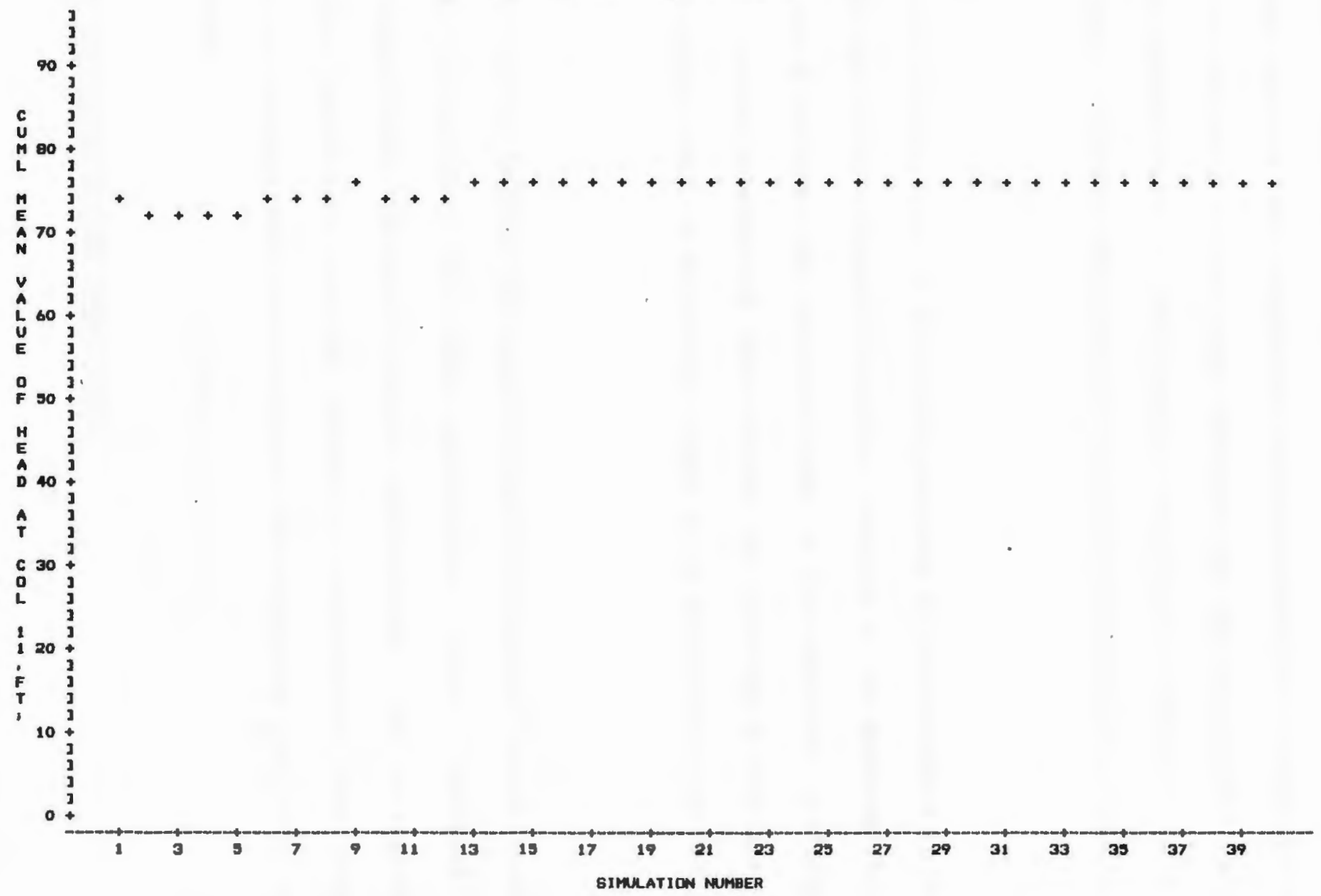

Figure 14 - Cummulative Mean of the Head Versus Simulation Number 
the mean value was within 3 percent of that value.

The effect of variations in region size is demonstrated in Figure 15. The maximum value of the normalized standard deviation of hydraulic head for any of the nodes in the region increases as the region size increased. The maximum value of the standard deviation of hydraulic head was normalized by dividing each value by the mean head loss in each block or grid.

The effect of variations in hydraulic gradient is demonstrated in Figure 16. The upper plot indicates a gradual increase in the maximum value of the standard deviation of hydraulic head as the hydraulic gradient increases. After normalizing these values by dividing by the mean head loss, however, there is no general trend as is demonstrated by the lower plot.

The relationship between this quantity and the standard deviation of the hydraulic conductivity is of more significance. Figure 17 presents this relationship which indicates a direct relationship. This is significant since the uncertainty in the hydraulic conductivity value directly affects our uncertainty in the value of the hydraulic head value within the region.

\section{Macroscopic Dispersivity Sensitivity}

The macroscopic or field scale longitudinal dispersivity has been shown to be a function of the standard deviation of the travel time. The 


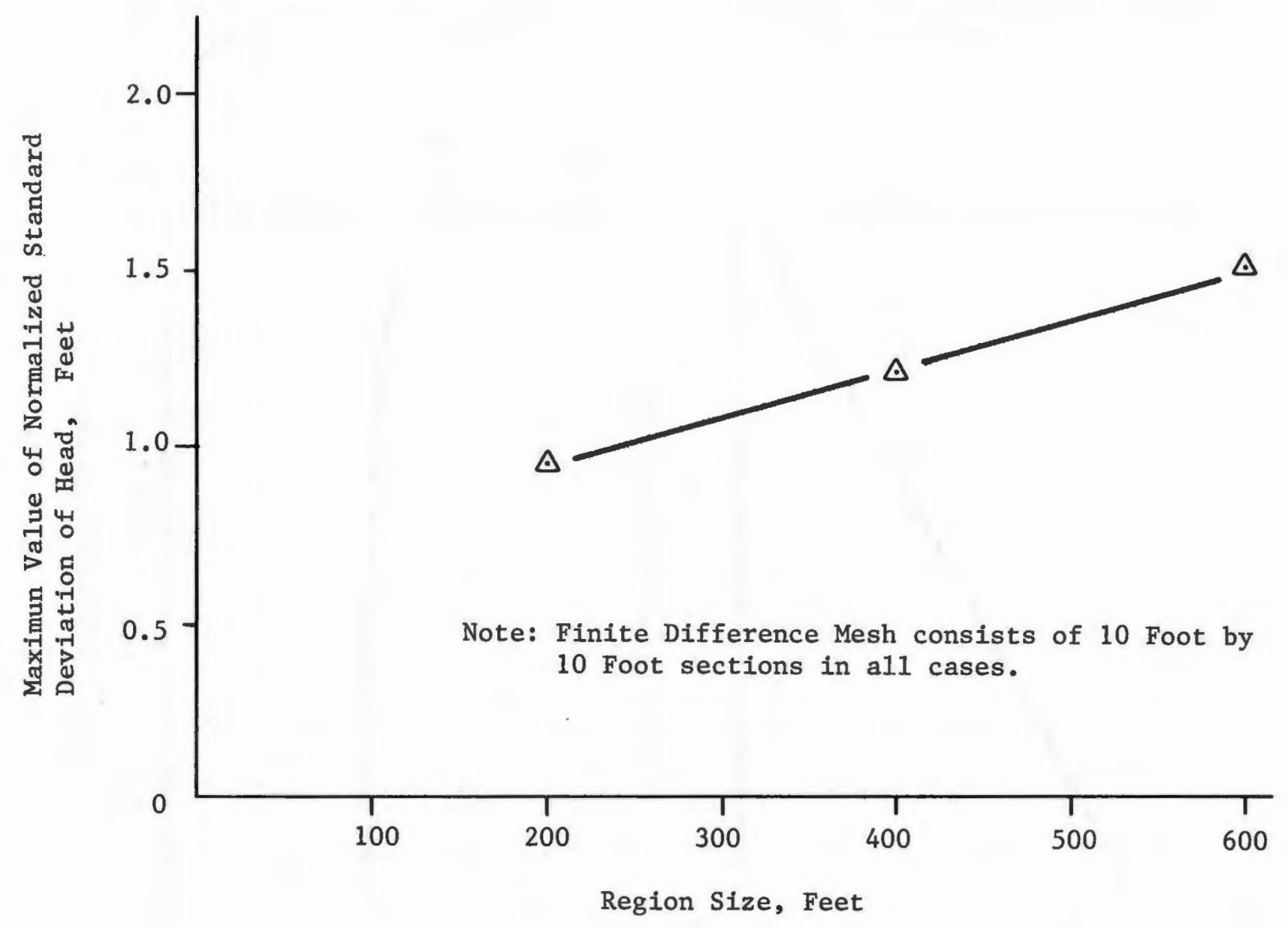



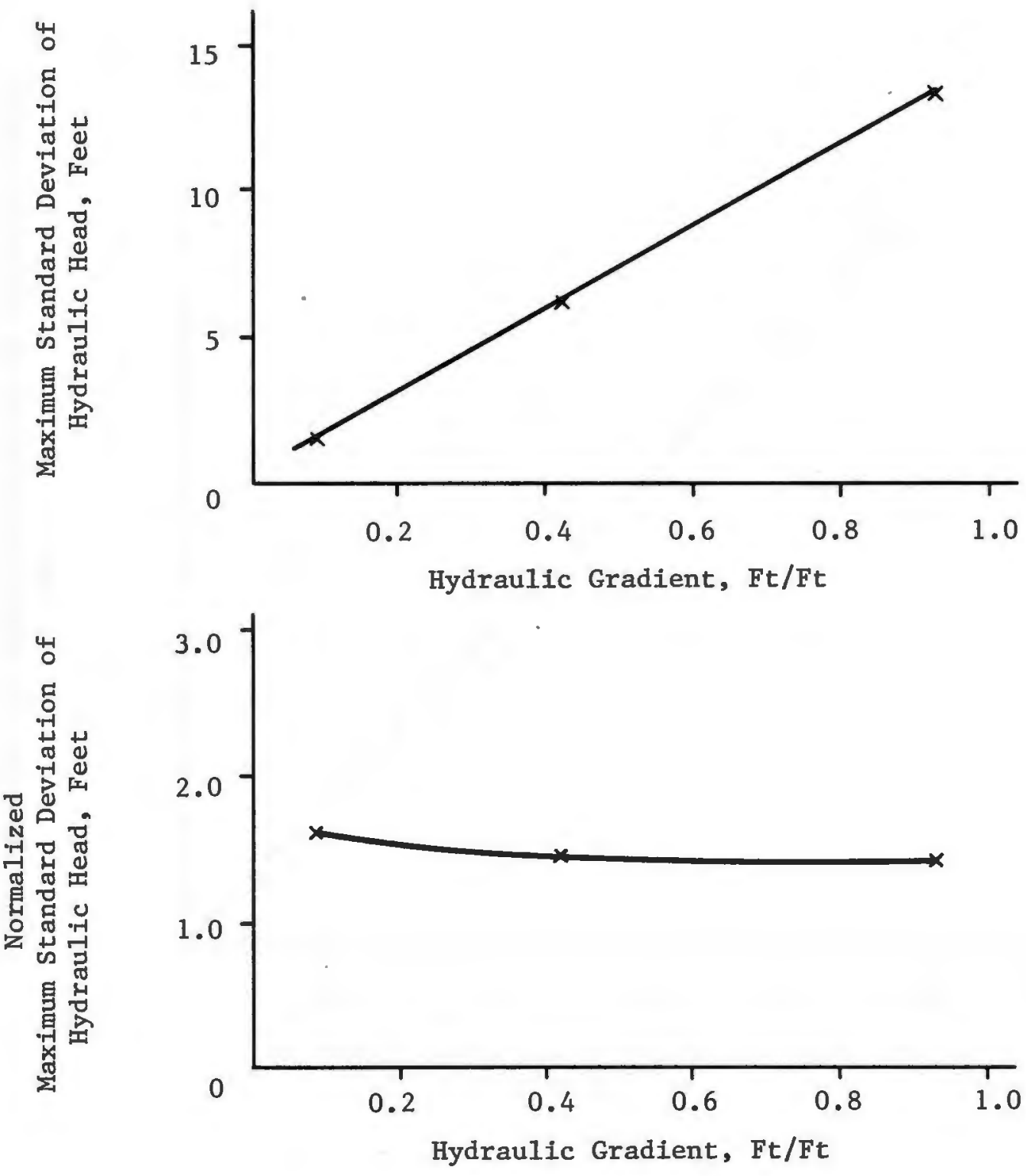

Figure 16 - Maximum Value of Standard Deviation of Head Versus Hydraulic Gradient 


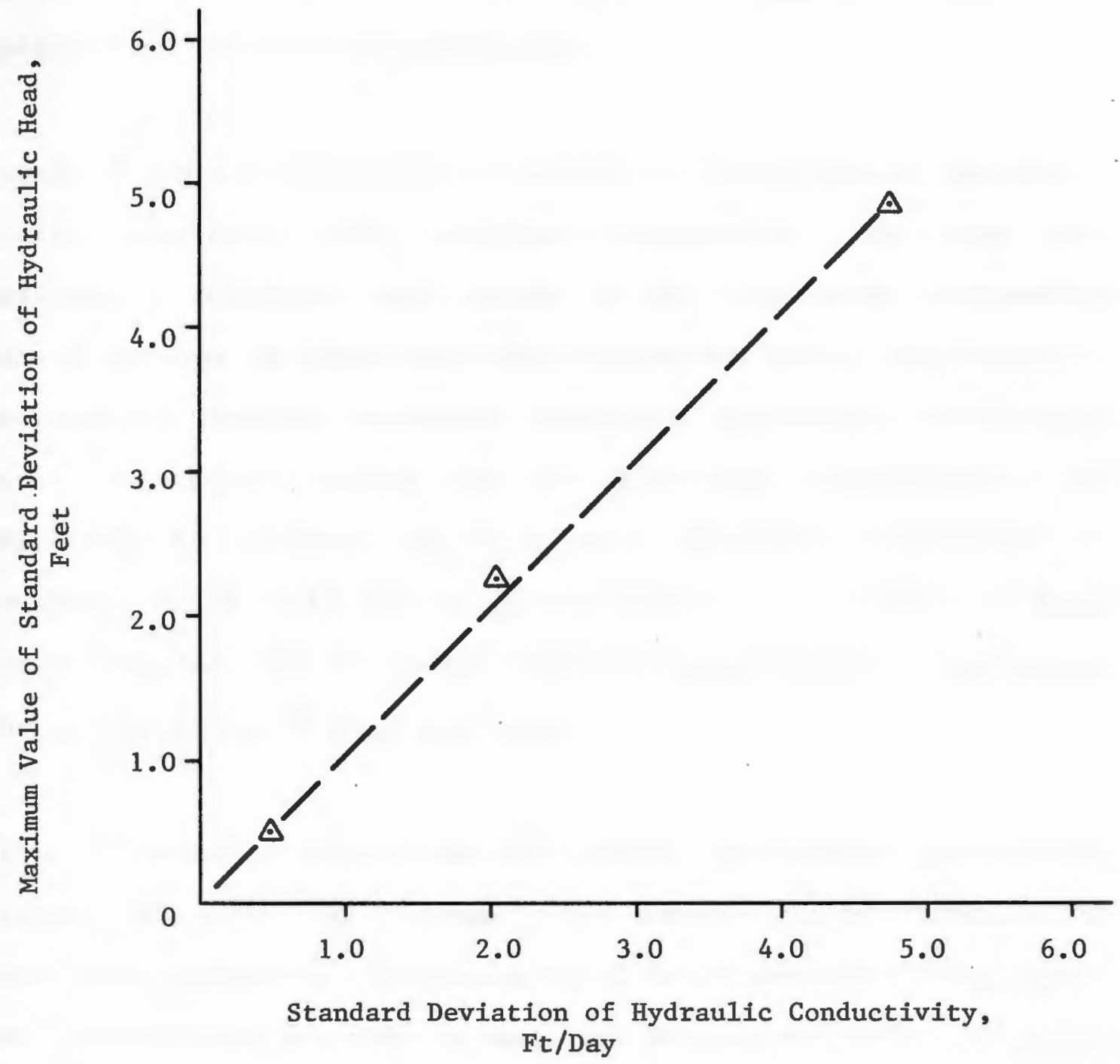

Figure 17 - Maximum Value of Standard Deviation of Head Versus Standard Deviation of Hydraulic Conductivity 
macroscopic transverse dispersivity has been shown to be a function of the standard deviation about the mean ending location perpendicular to the general flow direction. For the horizontal uniform flow case this may be taken as the standard deviation in the ending y coordinate. The sensitivity of the macroscope dispersivities to variations in region size, hydraulic gradient, time interval and standard deviation of hydraulic conductivity was investigated.

Figure 18 presents results of the effect of region size on the macroscopic longitudinal and transverse dispersivity. The upper plot indicates a relatively small change in the longitudinal dispersivity with an increase in region size and no consistent trend. The lower plot indicates a steadily decreasing transverse dispersivity with region size. The values plotted for the macroscopic dispersivities was determined by dividing the dispersion coefficients determined by equations 18 and 21 by the mean particle velocity in the region. These results suggest that a constant value of dispersivity is not reached during the transit of these particles.

Figure 19 presents the effects of a change in hydraulic gradient and Figure 20 the effects of a change in time interval for mass transport on these same parameters. The variation of both macroscopic longitudinal and transverse dispersivity is small and inconsistent due to the change in hydraulic gradient and time interval. The insensitivity of the observed dispersivity due to time interval variation is important since the computer time required to simulate particle transit is dependent on the time interval utilized. 


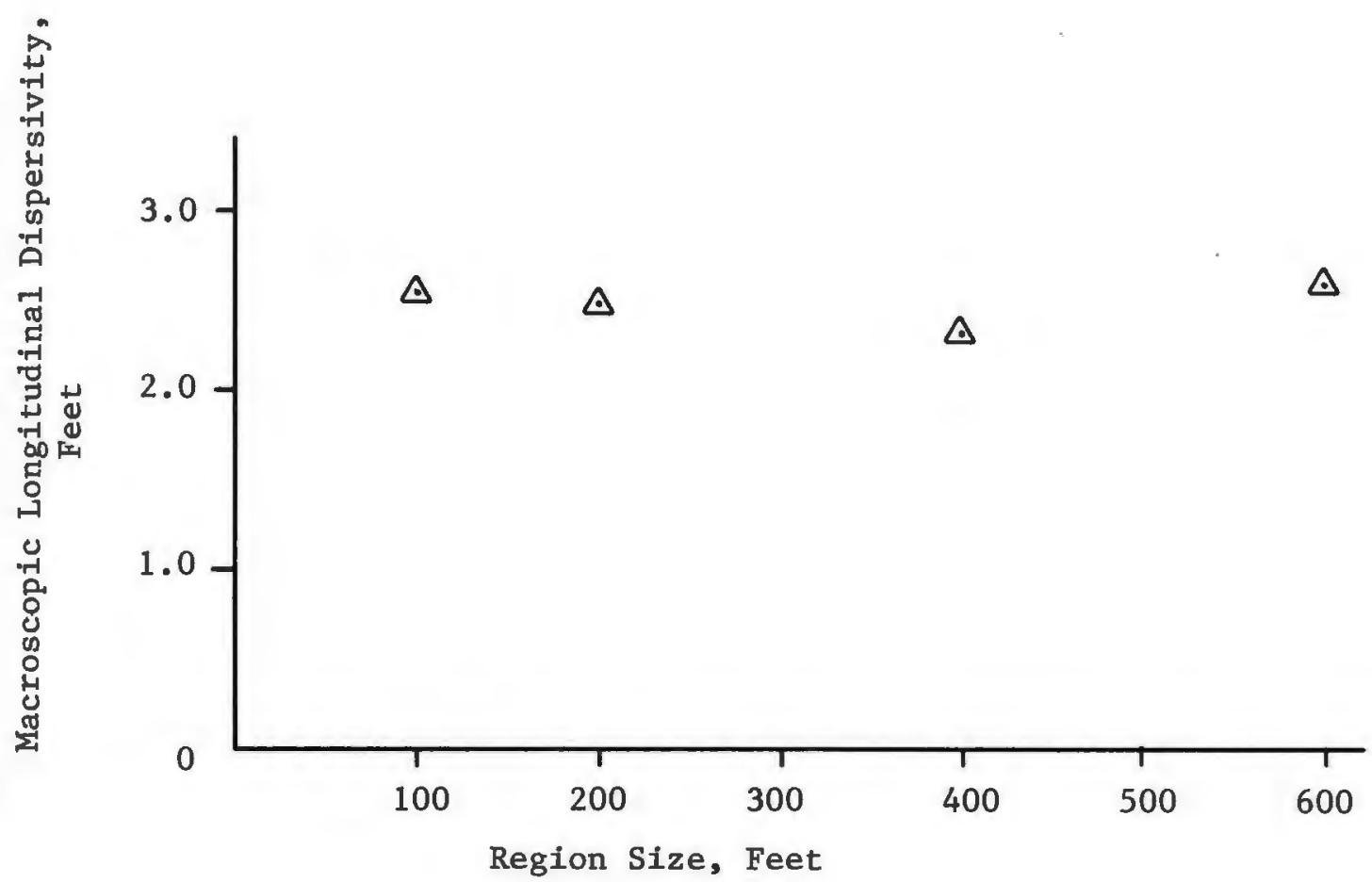

Note: Finite Difference Mesh consists of $10 \mathrm{Ft}$ by $10 \mathrm{Ft}$ Sections in all Cases.

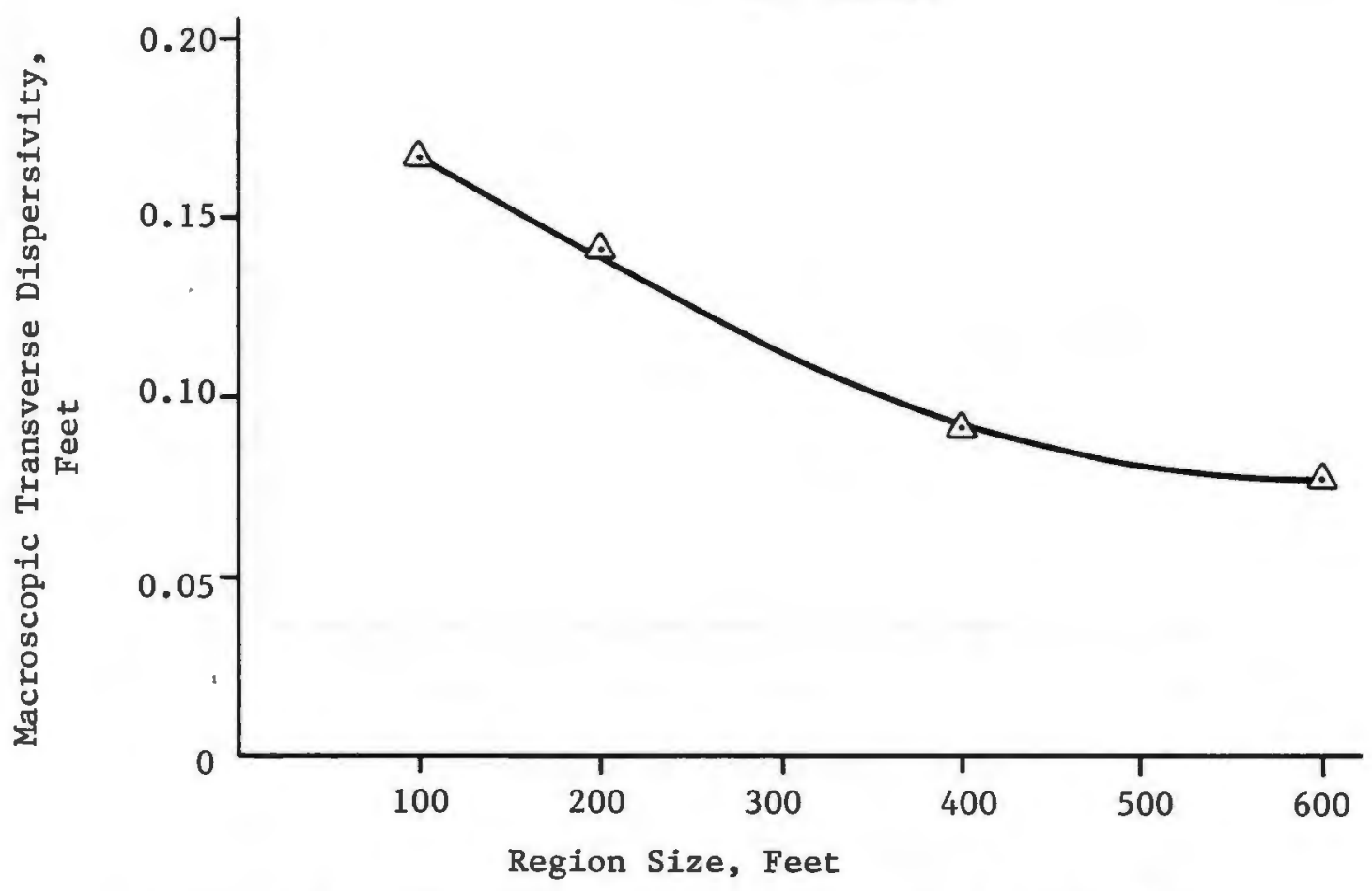

Figure 18 - Macroscopic Dispersivity Versus Region Size 

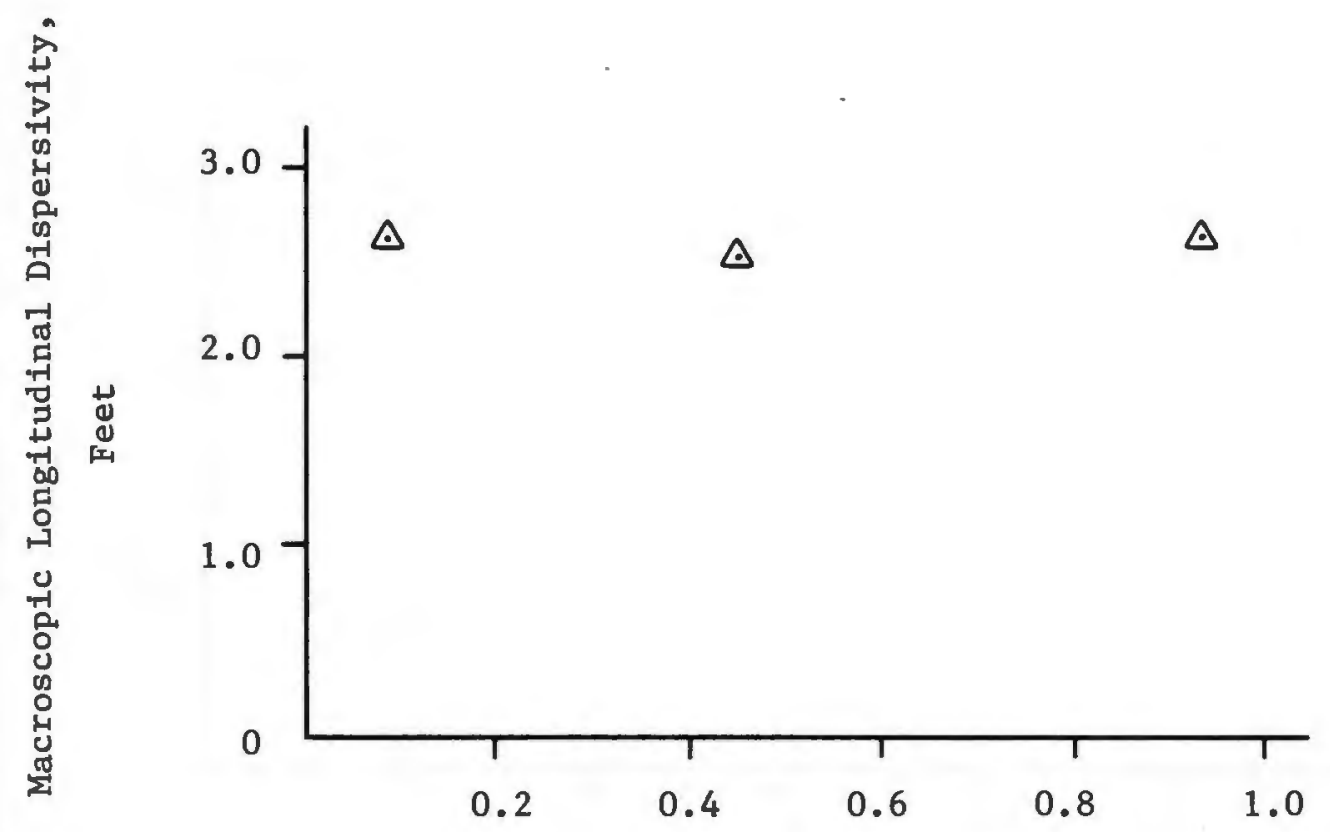

Hydraulic Gradient, Ft/Ft
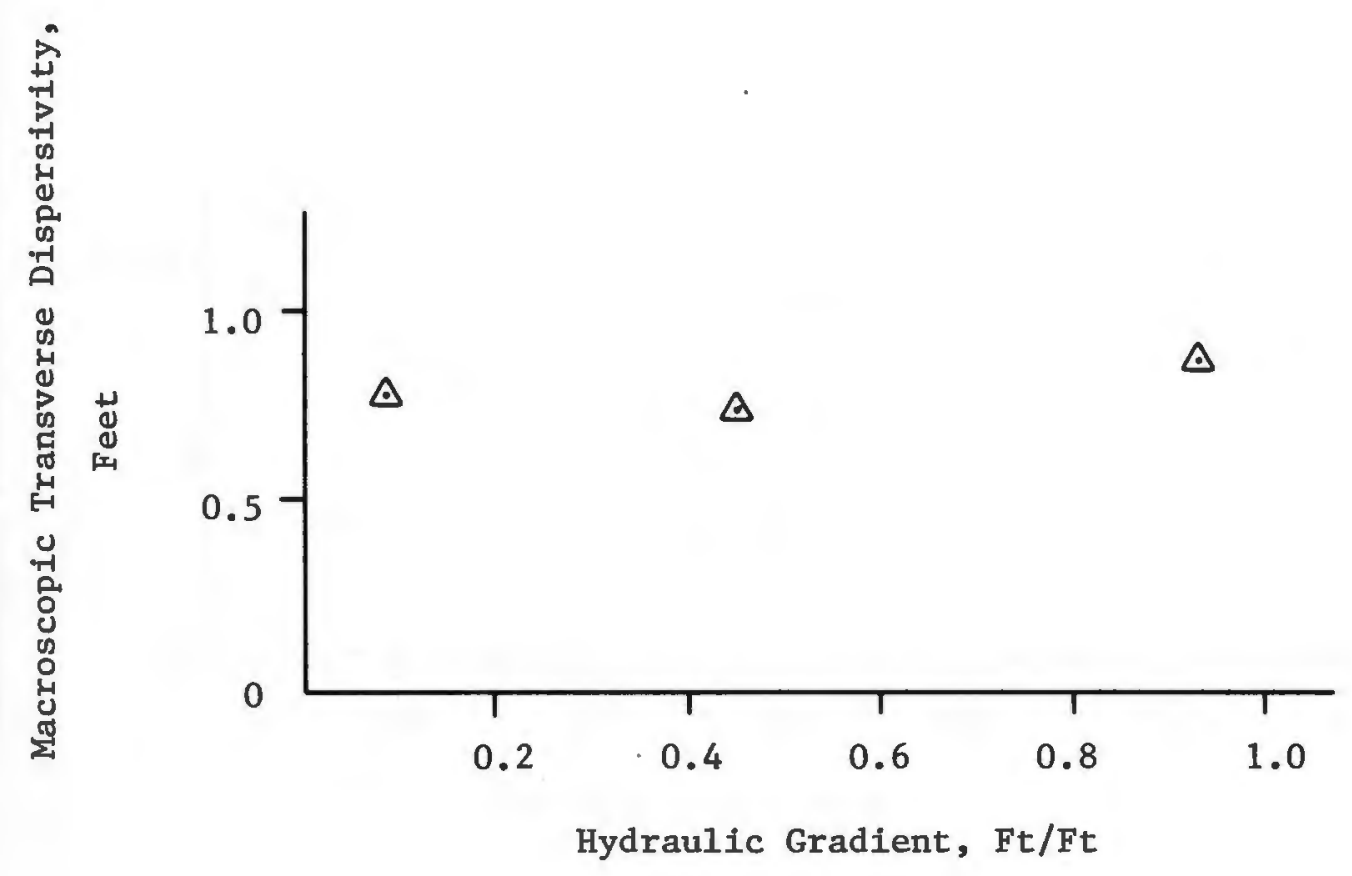

Figure 19 - Macroscopic Dispersivity Versus Hydraulic Gradient 
(5)

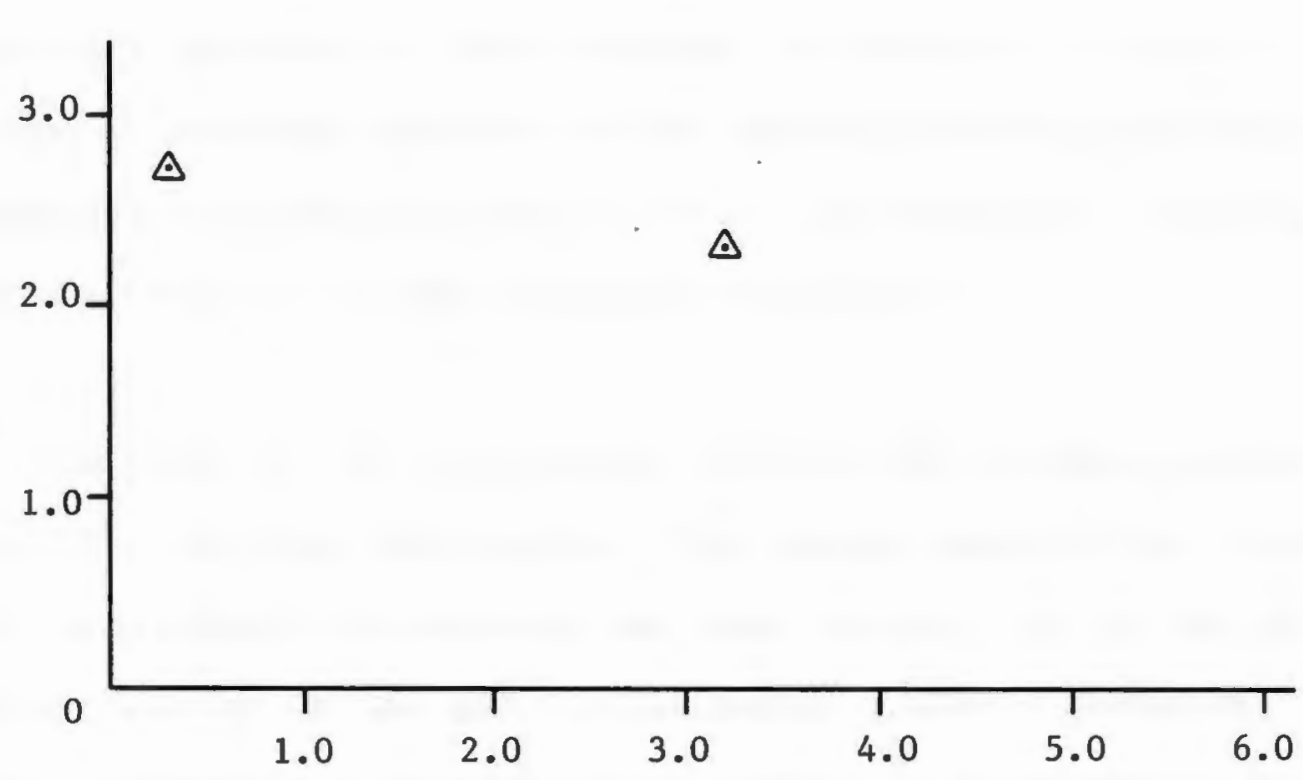

Time Interva1, Days

年

$\triangle$

$\Delta$

$\triangle$

0

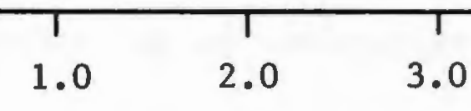

Time Interva1, Days

Figure 20 - Macroscopic Dispersivity Versus Time Interval 
The effect of a variation in the standard deviation of hydraulic conductivity is much more pronounced than these other variables. The results of a variation of this parameter are presented in Figure 21 . A reduction in standard deviation of the hydraulic conductivity from 2 to 0.5 resulted in a reduction from 2.5 to 0.3 in longitudinal dispersivity and from 0.14 to 0.05 on the transverse dispersivity.

The sensitivity of the macroscopic dispersivity on the microscopic dispersivity was also investigated. The typical range of lab or microscopic longitudinal dispersivisy has been reported (14) to be in the range of 0.1 to $20 \mathrm{~mm}$ for unconsolidated geologic sediments. Lab values of transverse dispersivity have likewise been reported to be 5 to 20 percent of the longitudinal dispersivity. Mass transport in regions of uniform flow with values of dispersivity near the extremes of this range was simulated. The initial simulation with both microscopic dispersivity values equal to zero and a singular constant value of hydraulic conductivity of five resulted in a macroscopic dispersivity of zero. A second simulation with microscopic dispersivities of 0.47 feet $(144 \mathrm{~mm})$ and 0.14 feet $(43 \mathrm{~mm})$ respectively, resulted in macroscopic dispersivities of 0.16 feet and 0.05 feet respectively. However when these same microscopic dispersivities are compared with the results presented in Figure 21, it is apparent that the variation of the microscopic dispersivities is of secondary importance relative to possible variations in hydraulic conductivity.

The sensitivity of the longitudinal dispersivity to changes in the shape of the flow region was also considered. Generally the greater the 


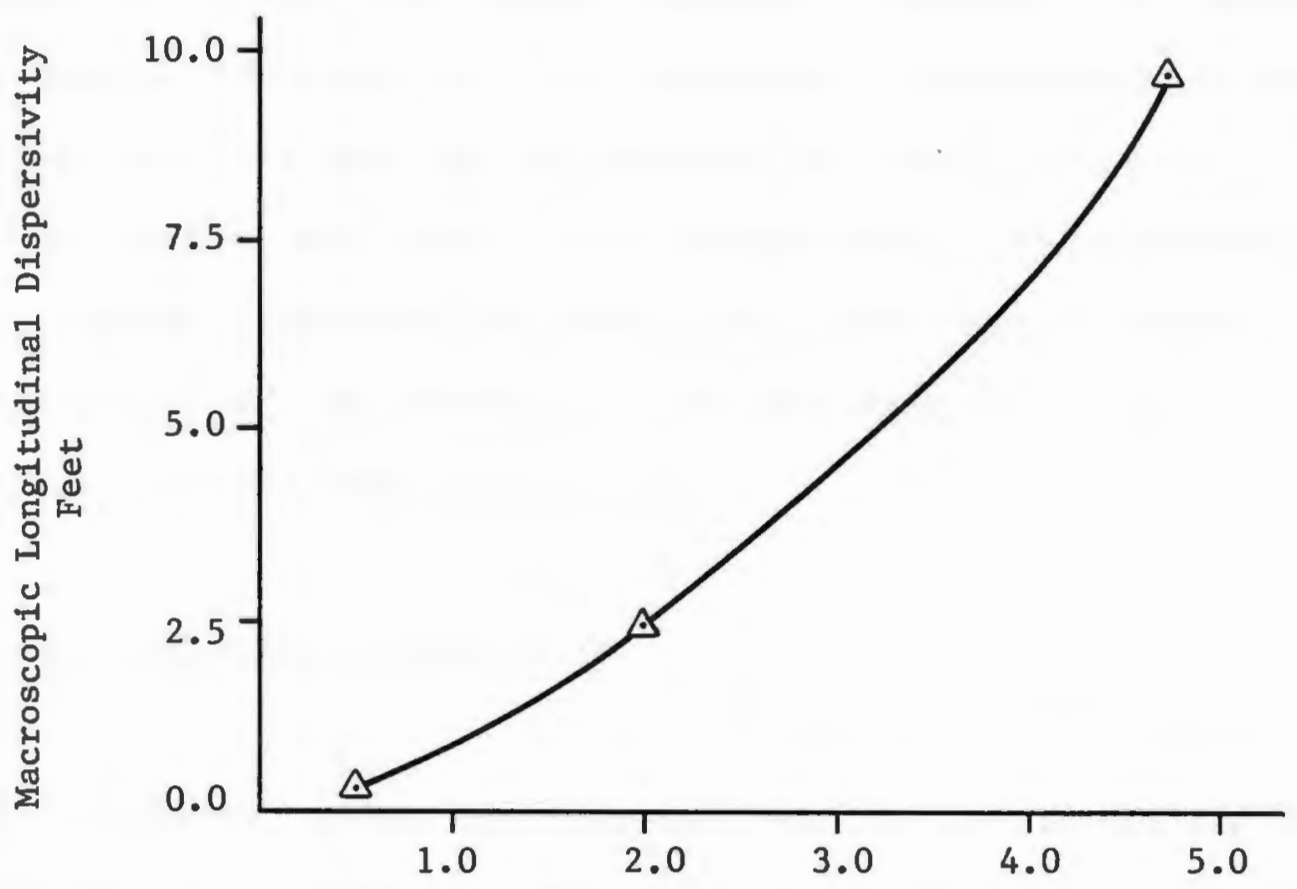

Standard Deviation of Hydraulic Conductivity, ft/day
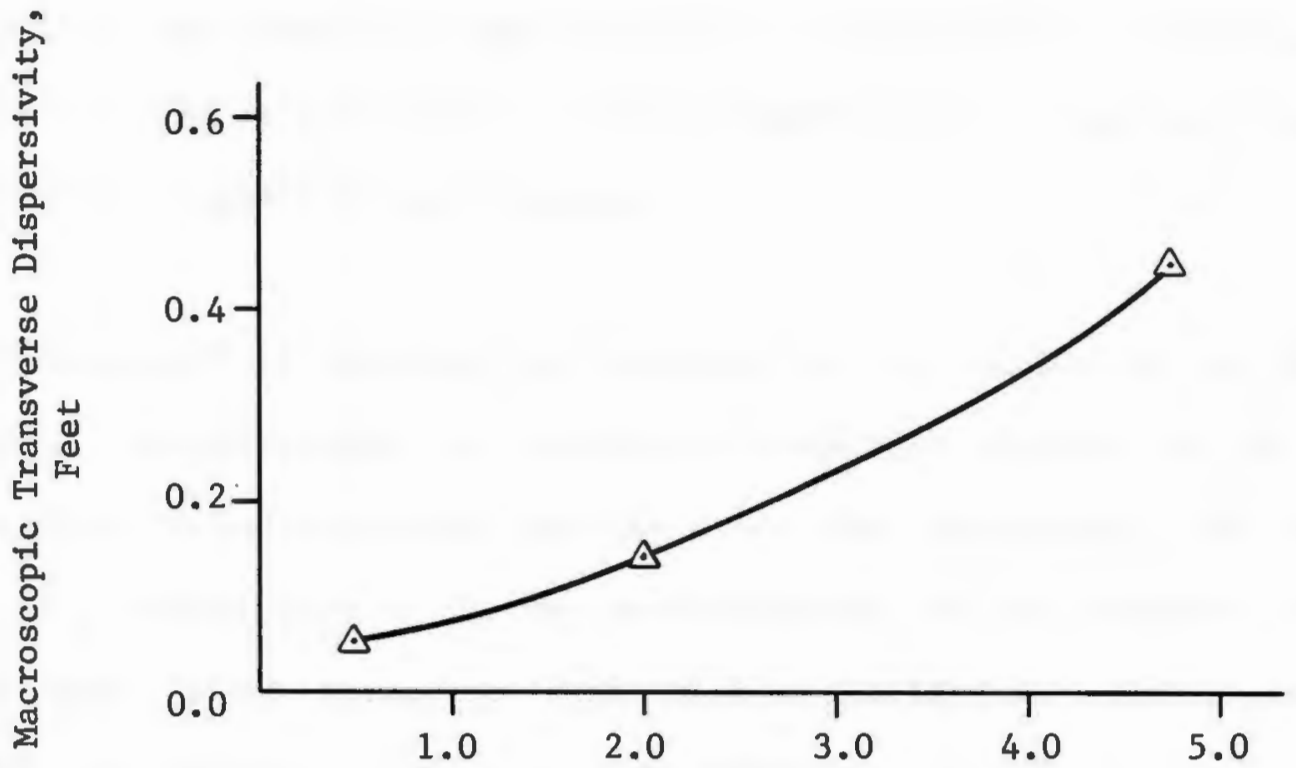

Standard Deviation of Hydraulic Conductivity, ft/day

Figure 21 - Macroscopic Dispersivity Versus Standard Deviation of Hydraulic Conductivity 
tortuosity of the flow region boundary conditions, the greater the macroscopic dispersivity. The macroscopic longitudinal dispersivity varied from 2.49 feet for the uniform flow case to 2.84 and 3.25 feet for the $S$ flow and cutoff cases respectively. For the three general flow regions considered the variation in macroscopic dispersivity was relatively small when compared to that resulting from a variation in the hydraulic conductivity distribution.

\section{Development of Confidence Limits}

The confidence which the engineer has in his prediction of flow quantity, travel times and dispersion is the major reason for utilizing these more sophisticated and complex solutions for field flow problems. If the engineer were not able to increase his confidence in his predictions there would be little reason to utilize these techniques. The nature of the output from this method lends itself to the development of confidence limits on the solution.

A methodology to utilize the knowledge of the variability of the flow region characteristics to establish confidence limits on the output utilizing probability and statistics has been formulated. The emphasis of this methodology is in the quantification of the characteristics of the major input parameter, hydraulic conductivity. Other parameters have been shown to be of lesser importance to the problem solution.

The procedure to develop the site specific soil parameters is essentially unchanged. Field programs are utilized to measure insitu 
soil parameters and to secure samples for laboratory testing. The field and laboratory tests will be analyzed so that a probability density function of the hydraulic conductivity may be formulated. Other parameters such as porosity, and laboratory dispersivity characteristics must also be developed.

These parameters are then the input to a Monte Carlo computer solution for flow quantity and travel time. The results of these Monte Carlo computer simulations may then be analyzed utilizing the knowledge of the underlying distribution of these results to develop confidence in the results.

In general the flow quantity and travel time results should be expected to conform to a normal distribution. This assumption is made on the basis of the Central Limit Theorem. The water particle movement may be considered the result of adding up the effects of many individual factors (hydraulic conductivity values). This assumption of normality has been only generally verified in this study, since numerous exceptions were observed.

The mean value of the flow quantity and travel time may then be estimated with confidence by the method described below. The results of one Monte Carlo simulation are presented in Figure 22. The characteristics of the flow region are also presented. The mean and standard deviation of the effective hydraulic conductivity, $\mathrm{K}_{\mathrm{q}}$, is 4.57 and 0.230 respectively. The magnitude of error of the mean with $95 \%$ confidence may be estimated by the following expression (26): 


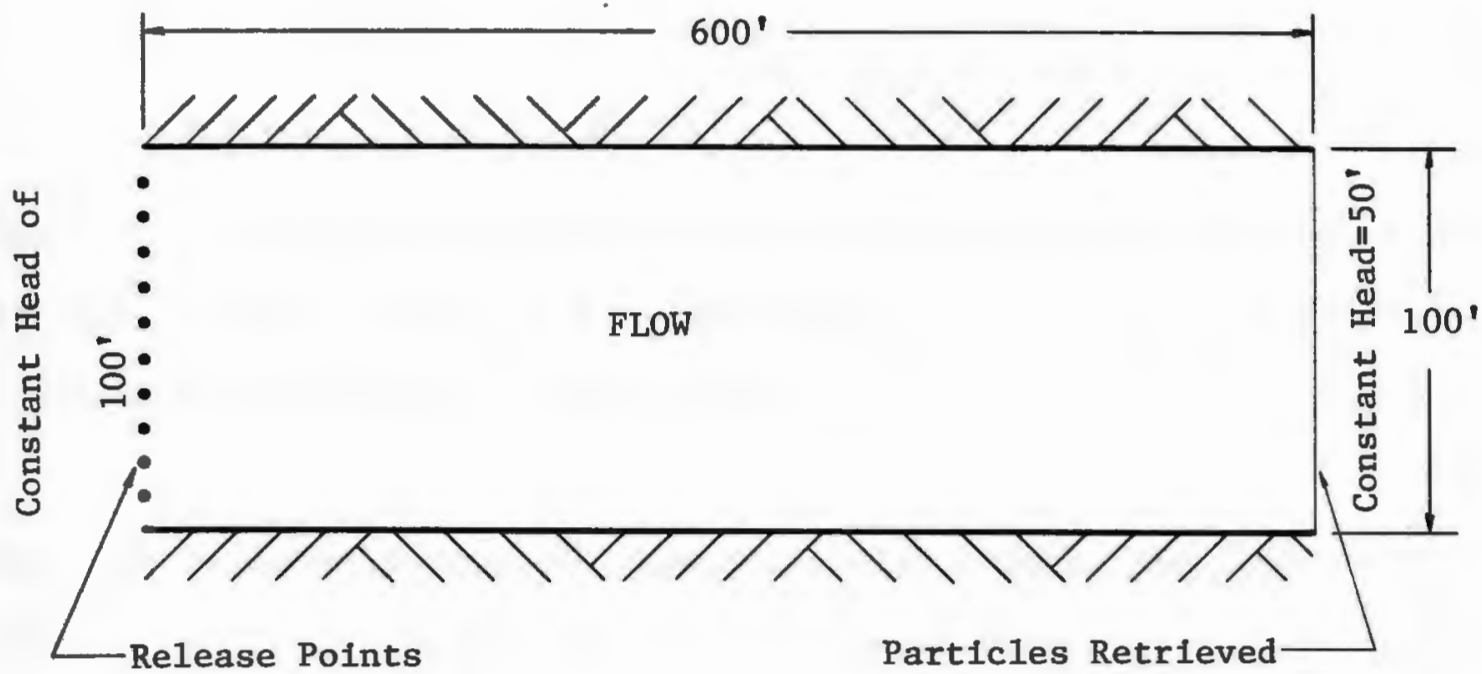

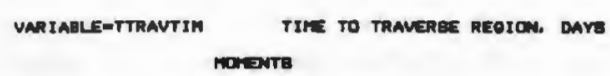

FILE 16, 40 atmLATIONB OF UMTFOM FLON IN $100 \mathrm{FT}$ oY $600 \mathrm{FT}$ REOSON

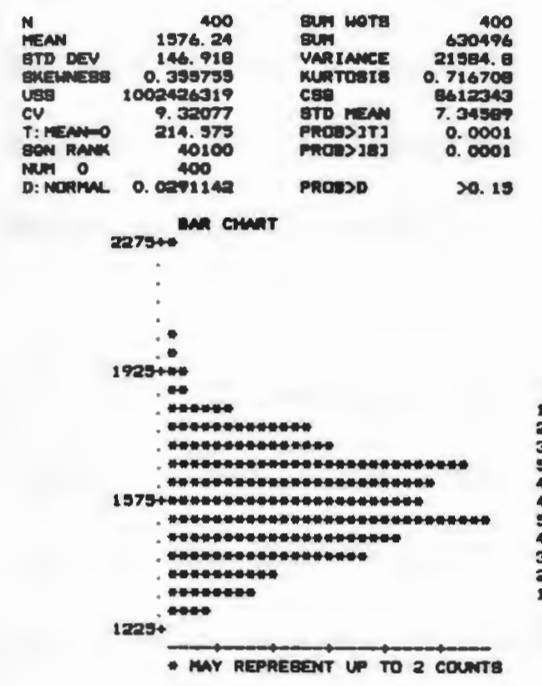
CUNHT ILES(DEFa)

EXTRERE:

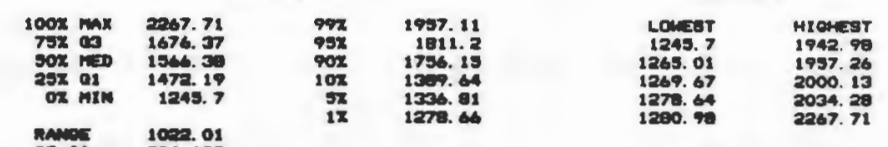

UMRIADLE-EFFKHYO

EFF HYD COND On BABIB of FLUX, FT/OA
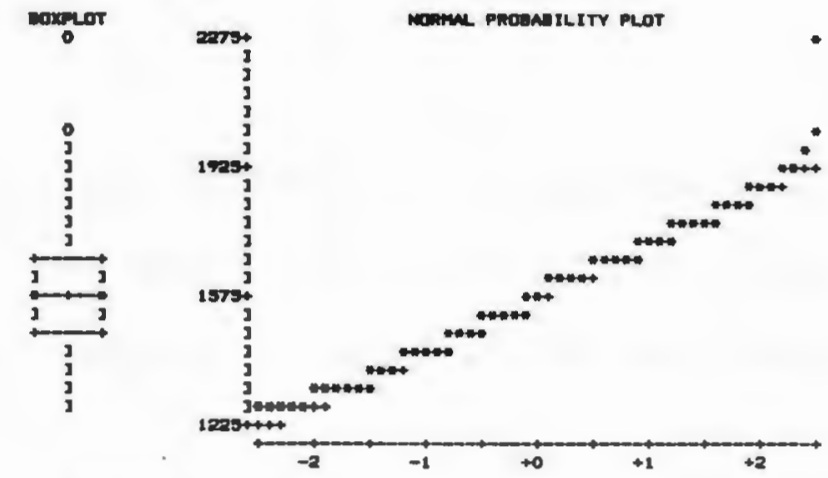

PRECUEACY TALE

\begin{tabular}{|c|c|c|c|}
\hline \multicolumn{4}{|c|}{ Monste } \\
\hline 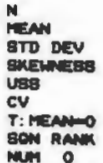 & 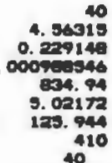 & 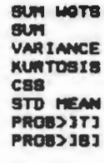 & $\begin{array}{r}100.926 \\
0.092509 \\
0.070460 \\
2.04789 \\
0.0340315 \\
0.0001 \\
0.0001\end{array}$ \\
\hline W: Neonmal & 0.969904 & PROSAM & 0.463 \\
\hline
\end{tabular}
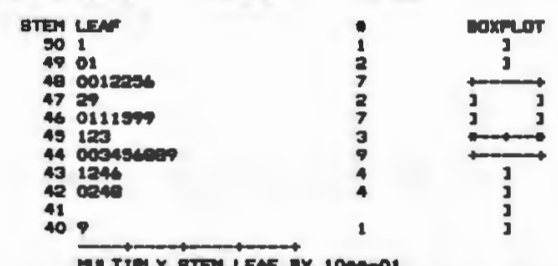

CUATILEB (DEFAA)
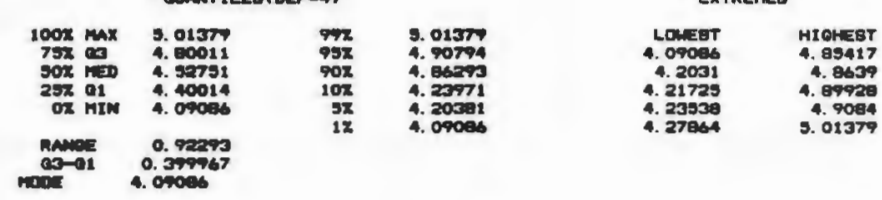

Figure 22 - Typical Output on Flow and Travel Time 


$$
E<t a / 2 \frac{s}{\sqrt{N}}=1.96 \cdot \frac{0.230}{\sqrt{40}}=0.071
$$

where $t_{\alpha / 2}$ is the value of the cumulative distribution function of the student $t$ distribution at $\alpha / 2$ significance, $s$ is the value of the standard deviation and $N$ is the sample size.

The mean value would then be expected to lie between the limits of the sample mean (4.57) plus or minus the error (0.07) i.e., 4.50 and 4.64 . The effective hydraulic conductivity, $\mathrm{K}_{\mathrm{q}}$, was calculated by dividing the flow by the mean hydraulic gradient and area. The flow rate may then be estimated with confidence limits of $95 \%$ by multiplying by these constants. In so doing the flow limits of 38 to $39.5 \mathrm{ft}^{3} /$ day were estimated with $95 \%$ confidence limits.

The same methodology may be utilized to develop confidence limits on the travel times. The mean and standard deviation of the travel time is 1576.2 and 146.9 days. The magnitude of error of the mean with $95 \%$ confidence may be estimated as:

$$
E<\frac{1.96(146.9)}{\sqrt{400}}=14.4 \text { days }
$$

The mean value of travel time is therefore expected to lie between 1562 and 1591 days.

The standard deviation of the travel time may also be estimated with confidence by the expressions given below (26): 


$$
\frac{s}{1+\frac{z_{\alpha / 2}}{\sqrt{2 N}}}<\sigma<\frac{s}{1-\frac{z_{\alpha / 2}}{\sqrt{2 N}}}
$$

where $Z_{\alpha / 2}$ is the value of the cumulative density function at a significant of $\alpha / 2$. Utilizing this equation results in the following:

$$
\frac{146.9}{1+\frac{1.96}{\sqrt{2(400)}}}<\sigma<\frac{146.9}{1-\frac{1.96}{\sqrt{2(400)}}}
$$

which reduces to:

$$
137.4<\sigma<157.8
$$

The water particles are expected to follow a normal distribution so that a hypothetical breakthrough curve may be developed for the extremes of these parameters. The result of such an exercise is presented in Figure 23. This allows the engineer to estimate not only the mean arrival time with confidence but also the distribution of travel times. 


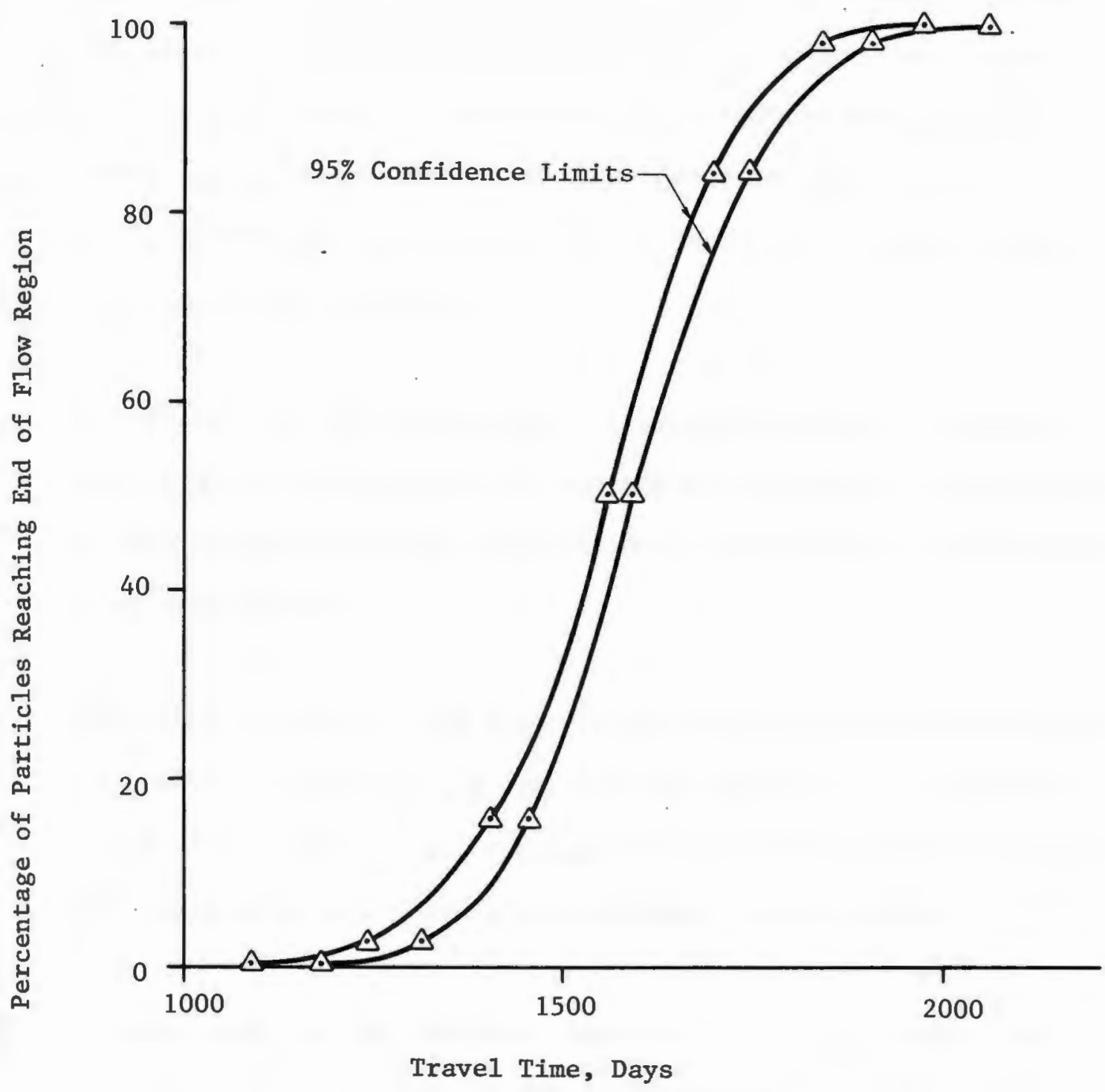

Figure 23 - Hypothetical Breakthrough Curve with Confidence Limits 
CONCLUSIONS

The methodology outlined herein which utilizes a stochastic nearest neighbor model and a log normal PDF technique to simulate the variation in hydraulic conductivity (permeability) is useful in incorporating the variability in flow regions into flow calculations. The following conclusions concerning the results of the Monte Carlo simulations for steady state flow may be drawn:

1. The effects of the boundaries, of stacking blocks of internally autocorrelated subregions and the log transformation necessitated by this technique did not severely erode the integral scale for the cases considered.

2. The geometric mean is the best estimate of the effective hydraulic conductivity value computed either on the basis of flow quantity or travel time. This is true for uniform as well as the more tortuous flow conditions considered after applying a shape factor.

3. The magnitude of the standard deviation of the hydraulic head at any point in the flow region is a function of the standard deviation of the hydraulic conductivity, the region size and the distance from the boundary.

4. The flow quantity and travel time to traverse the region cannot always be assumed normally distributed. This may be due to the limited size of the regions considered in this study. However, 
when testing confirms normality, confidence limits for the mean and standard deviation may be developed. These estimates could provide the necessary input for economic studies of alternates when tempered with engineering judgement.

5. The macroscopic lateral and transverse dispersivities did not consistently reach constant values within the distance studied. The magnitude of the macroscopic dispersivity was more dependent on distributions of hydraulic conductivity than other parameters considered including variations in the microscopic dispersivity. 
APPENDIX I - REFERENCES

1. Ahlstrom, S.W., H. P. Foote, R. C. Arnett, C. R. Cole, and R. J. Serne, "Multicomponent mass transport model: Theory and numerical implementation (discrete-parcel-random walk version)," Rep. BNWL-2127, Battelle Pacific Northwest Lab., Richland, Washington, 1977.

2. Aldinger, P. B., "Groundwater Flow Simulation by a Stochastic Representation of Soil," thesis presented to the University of Rhode Island at Kingston, $\mathrm{RI}$ in 1983, in partial fulfillment of the requirements for the degree of Doctor of Philosophy.

3. Aldinger, P. B., "Applications of a Computer Model to Simulate Groundwater Flow in a Stochastic Medium," J. of the Geotechnical Division, ASCE, to be submitted.

4. Bakr, A.A., "Stochastic analysis of the effect of spatial variations in hydraulic conductivity on groundwater flow," PhD dissertation presented to the New Mexico Institute of Mining and Technology at Soccorro in 1976 in partial fulfillment of the requirements for the degree of Doctor of Philosophy.

5. Bartlett, M.S., The Statistical Analysis of Spatial Pattern, 91 pp., Chapman and Hall, London, 1975.

6. Benjamin, J.R., and Cornell, C.A., Probability Statistics and Decision for Civil Engineers, McGraw Hill, New York, 1970. 
7. Besag, J. "Spatial interaction and the statistical analysis of lattice systems," J. Roy. Statist. Soc., B 36, 192-237, 1974.

8. Bouwer, H., "Planning and interpreting soil permeability measurements," J. Irrig. Drain Div., ASCE, Vol. 95, 391-402, 1969.

9. Brook, D., "On the Distinction between the conditional probability and the joint probability approaches in the specification of nearest neighborhood systems," Biometrika, Vol. 51, 481-483, 1964.

10. Butler, Stanley, S. and Gundlach, David, L., "Discharge and Travel Time for Ground-Water Conduits", J. of the Irrigation and Drainage Division, ASCE, Vo1. 100, No. IR1, Mar. 1974.

11. Casagrande, Arthur, "Seepage Through Dams", Journal of the New England Water Works, Vol 51, Jun. 1937.

12. Cedergren, H.R., Seepage, Drainage and Flow Nets. New York: John Wiley and Sons, Inc., 1967.

13. Freeze, R.A. A stochastic-conceptual analysis of one dimensional groundwater flow in nonuniform homogeneous media, Water Resour. Res. Vo1 II (5), 725-741, 1975 .

14. Freeze, R.A. and Cherry, J.A., Groundwater, Prentice-Ha11, New Jersey, 604 p. 1979. 
15. Fried, J.J., Groundwater Pollution, Elsevier Scientific, Amsterdam, 330 p. 1975.

16. Gelhar, L.W., "Effects of hydraulic conductivity variations in groundwater flows," in Proceedings, Second International IAHR Symposium on Stochastic Hydraulic, International Association of Hydraulic Research, Lund, Sweden, 1977.

17. Gelhar, L.W., A. L. Gutjahr, and R. L. Naff, "Stochastic Analysis of Macrodispersion in a Stratified Aquifer," Water Resources Research, Vol 15, No. 6, Dec. 1979.

18. Gutjahr, A.L., L.W. Gelhar, A.A. Bakr, and J.R. MacMillan, "Stochastic analysis of spatial variability in subsurface flows, 2, Evaluation and Application", Water Resour. Res, Vol. 14 (5), 953$959,1978$.

19. Harr, M.E. Groundwater and Seepage, New York: McGraw-Hill Book Co., Inc. 1962 .

20. Harr, M.E. Mechanics of particulate Media, A Probabilistic Approach, McGraw-Hill Book Co., Inc. 1977.

21. Kelly, William E., personal communication, 1982 .

22. Koch, G.S. Jr., and R.F. Link, Statistical Analysis of Geological Data, Vol. 1 and 2, Dover Publications, Inc., New York, 1970. 
23. Krumbein, W.C., "Trend Surface Analysis of Contour-Type Maps with Irregular Control-Point Spacing", Journal of Geophysical Research, Vol. 64, No. 7, July 1959.

24. Lumb, P., "Application of Statistics in Soil Mechanics" Chapter 3 in "New Horizons in Soil Mechanics" Ed. by I.K. Lee, American Elsevier Publishing Co., Inc., N.Y., 1974.

25. Martin, R., "On spatial dependence, bias and use of first spatial differences in Regression Analysis", Area, Vol. 6, 185-194, 1974.

26. Miller, I., and Freund, J.E., Probability and Statistics for Engineers, Prentice-Hall, Inc., Englewood Cliffs, New Jersey, 1977.

27. Prickett, T.A. "Modeling Techniques for Groundwater Evaluation" In Advances In Hydroscience, Vol. 10, Academic Press, New York, 1975.

28. Prickett, T.A.; Naymick, T.G. and Lonnquist, C.G., A Randon Walk Solute Transport Model For Selected Groundwater Quality Evaluations by Illinois State Water Survey, Bulletin 65, 1981.

29. Reiter, P.F., "A Computer Study of the Correlation Between Aquifier Hydraulic and Aquifier Electric Properties," A thesis submitted in partial fulfillment of the requirements for the degree of Master of Science in Civil and Environmental Engineering, University of Rhode Is land, 1980 . 
30. Sauty, J.P., "Computer Simulation of Pollution Front Movement", Conference Presentation in France, 1976.

31. Smith, L., "A stochastic analysis of steady-state groundwater flow in a bounded domain", dissertation presented to the University of British Columbia in Vancouver, Canada in 1978, in partial fulfullment of the requirements for the degree of Doctor of Philosophy.

32. Smith, L., "Spatial Variability of flow Parameters in a Stratified Sand," Mathematical Geology, Vol. 13, No. 1, 1981.

33. Smith, L. and R.A. Freeze, "Stochastic Analysis of Steady State Groundwater Flow in a Bounded Domain, 1, One-demensional Simulations", Water Resources Research, Vol. 15, 521-528, 1979.

34. Smith I., and R.A. Freeze, "Stochastic Analysis of Steady State Groundwater Flow Research in a Bounded Domain, 2, Two Dimensional Simulations", Water Resources Research, Vol. 15, 1543-1559, 1979.

35. Smith, L. and F. Schwartz, "Mass Transport, 1, A Stochastic Analysis of Macroscopic Dispersion", Water Resources Research, Vol. 16, No. 2, April, 1980.

36. Tabba, M.M. and Yong, R.M., "Mapping and Predicting Soil Properties: Theory", Journal of the Engineering Mechanics Division, ASCE, Vol. 107, No. EM5, Proc. Paper 16534, Oct., 1981, pp. 773-791. 
37. Tabba, M.M. and Yong, R.N. "Mapping and Predicting Soil Properties: Applications," Journal of the Engineering Mechanics Division, ASCE, Vol. 107, No. EM5, Proc. Paper 16533, Oct., 1981, pp. 795-811.

38. Trescott, P.C. Pinder, G.F. and Larson, S.P., 1976, Techniques of Water-Resources Investigations of the U.S.G.S., Chapt. Cl. FiniteDifference Model for Aquifer Simulation in Two-Dimensions with Results of Numerical Experiments", U.S. Gov. Printing Office.

39. Wang, H.F. and M.P. Anderson, Introduction to Groundwater Modeling, Finite Difference and Finite Element Methods, W.H. Freeman and Co., San Francisco, 1982.

40. Warren, J.E. and M.S. Price, "Flow in Homogeneous Porous Media", Society of Petroleum Engineers, Sept. 1961.

41. Warren, J.E. and F.F. Skiba, "Macroscropic Dispersion," Soc. Petrol. Eng. J. 215-230, Sept., 1964.

42. Whitten, E.H.T. Compositional Trends in a granite model variation and ghost-stratigraphy in part of the Donegal Granite", Eire: Jour. Geophys. Research, Vol. 64, p. 835-848, 1959.

43. Whittle, P., "Stochastic processes in several dimenstions", Bull. Int. Statist. Inst., Vol. 40, 974-994, 1963. 
APPENDIX II - NOTATION

The following symbols are used in this paper:

$\mathrm{D}_{\mathrm{L}} \quad$ = macroscopic longitudinal dispersion coefficient

$\mathrm{D}_{\mathrm{T}} \quad=$ macroscopic transverse dispersion coefficient

$\mathrm{D}_{50} \quad=50 \%$ particle size or mean particle size

DELT = time increment, days

DL = longitudinal displacement

DLC = microscopic longitudinal dispersion coefficient

DSPRAT = transverse to longitudinal dispersivity ratio

DT = transverse displacement

DTC = microscopic transverse dispersion coefficient

E $\quad$ magnitude of error

$\mathrm{F}(\mathrm{K}) \quad$ = probability density function of $K$

G = variable relating standard deviations of $\mathrm{Y}$ to $\mathrm{K}$ 
h

${ }^{\mathbf{h}_{\mathrm{I}}}$

H

$\mathrm{H}_{\mathbf{L}}$

$\mathbf{H}_{\mathbf{u}}$

i

$\overline{\mathbf{K}}$

$\mathbf{K}_{\mathbf{a}}$

$\mathrm{K}_{8}$

$\mathbf{K}_{\mathbf{h}}$

$\mathbf{K}_{\mathbf{q}}$
= hydraulic head

= hydraulic head loss

= height of flow region

= hydraulic head at downgradient boundary

= hydraulic head at upgradient boundary

= row number of finite difference grid system

= identity matrix

$=$ column number of finite difference grid system

= hydraulic conductivity (permeability) at nodal point $i, j$

= mean hydraulic conductivity

= arithmatic mean of hydraulic conductivity

= geometric mean of hydraulic conductivity

= harmonic mean of hydraulic conductivity

= effective hydraulic conductivity on basis of flow 
$\mathbf{K}_{\mathbf{t}}$

$\mathbf{L}$

$\mathrm{L}_{\mathrm{T}}$

n

$\mathrm{N}$

$\mathrm{N}_{\mathrm{D}}$

$\mathrm{N}_{\mathbf{F}}$

RANOL, RANOT

$\mathbf{s}$

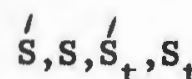

$\overline{\mathbf{t}}$

$t_{\alpha / 2}$

$\overline{\mathrm{u}}$
= effective hydraulic conductivity on basis of travel time

= length over which head loss occurs

= length over which water travels in flow simulation

= porosity

= sample size

$=$ number of equipotential drops in flow net

$=$ number of flow lines in flow net

= random number from normal distribution

= sample standard deviation

= shape factors

$=$ mean travel time

= cumulative distribution function of student $t$ distribution at $\alpha / 2$ significance

= mean particle velocity 
$\mathbf{v}$

W

$\mathbf{x}$

$\mathbf{X}$

$\alpha_{x}, \alpha_{y}$

$\alpha$

$\delta$

$\varepsilon$

$\lambda$

n

$\rho$

$\sigma$
= velocity of water

= weighting factor matrix

$=$ coordinate

= distance of total particle travel

= coordinate

= autoregressive parameters

= significance level

= macroscopic dispersivity

$=$ random number matrix

= integral scale

$=$ constant which relates $\sigma_{K}$ to $\sigma_{\sigma_{Y}}$

$=$ correlation coefficient

= standard deviation 
SECTION II

GROUNDWATER FLOW IN A STOCHASTIC MEDIUM:

APPLICATIONS OF COMPUTER MODEL

BY

PAUL BRUCE ALDINGER ${ }^{1}$, M. ASCE

\begin{abstract}
The use of a computer model which characterizes flow in a porous medium as a stochastic process is demonstrated on two field problems. The computer model allows the hydraulic conductivity (permeability) to be varied within the blocks of the finite difference mesh according to a predetermined autocorrelation structure and a $10 \mathrm{~g}$ normal probability density function. Mass transport is simulated as a combination of advection and microscopic or particle scale dispersion. The model allows development of confidence in the flow rate and travel times within the region.
\end{abstract}

INTRODUCTION

Geotechnical engineers are asked to make predictions of groundwater flow in a variety of boundary conditions and soil media. Sometimes there is considerable data available, but more often the problem is so large, the boundary conditions and soil medium so complex, that the resources available can not be expected to identify in detail the conditions insitu.

Nevertheless, predictions are required. The geotechnical engineer has

${ }^{1}$ Principal Geotechnical Engineer, Department Manager, CE Maguire, Inc. Providence, Rhode Island 
met that need by idealizing boundary conditions and soil media characteristics and making conservative assumptions as necessary to develop predictions.Sensitivity analyses have also been performed to study the effects varying of input parameters have on predictions.

Recently new techniques have been introduced which allow more complex and realistic input parameter characterization. In addition, other techniques have been utilized to analyze the results of numerical simulations. One of these tools, the use of probability and statistics, is utilized to estimate confidence limits for the prediction resulting from such analyses.

A word of caution is always necessary when discussing the use of probability and statistics in engineering problems. Too often the user forgets that these results are only estimates and that uncertainty still exists. These techniques do help develop confidence in one's predictions, but like all engineering tools, they must be weighted with engineering judgement.

This paper is the second of two papers. The first paper detailed the development of a computer model which uses a Monte Carlo technique, a finite difference numerical solution and a stochastic representation of the soil medium to simulate groundwater flow (1). This paper presents two applications of this computer model to study a typical groundwater flow problem. The first application is an actual situation in Rhode Island, the second is a hypothetical problem. 


\section{BACKGROUND}

A two dimensional characterization of the flow region has been chosen for several reasons. The practicing engineer rarely has sufficient information to make a three-dimensional model worth the expense of developing it. The practicing engineer is therefore more familiar with two dimensional representations. In addition, the computational effort required for stochastic three dimensional solutions would be enormous. Perhaps the techniques will be expanded to the three-dimensional case when further advances in computers allow and researchers consider the effort worthwhile.

For problems considered in this paper, steady state conditions are assumed. The first considers flow beneath a dike and the second flow away from a waste disposal lagoon. The flow region has been assumed rectangular for simplicity although other region shapes could be utilized.

The practicing engineer's primary analysis techniques until quite recently have been analytical solutions and flow nets. Analytical solutions are exact solutions to the flow conditions and have been developed to solve many flow situations (6). Where appropriate, these techniques provide reliable answers. These techniques suffer from their lack of flexibility and the need to make restrictive simplifying assumptions for boundary conditions and media parameters. In general, regions must be assumed homogeneous and isotropic. Anisotropy can generally be included by performing a transformation. 
Perhaps the technique most widely used today is the flow net. This technique has been available for nearly half a century and been utilized extensively $(3,4)$. Its greatest advantage is its simplicity and ease of use. The technique suffers from its limited capacity to vary the soil's hydraulic conductivity and freewater surface, however.

Numerical methods allow more realistic problem formulations. In most cases, however, the problem must be simplified into regions where the soil media have constant hydraulic conductivity. The companion paper (2) discusses the problems with this type of approach as well as other more realistic modifications.

The computer model discussed in the companion paper (2) requires input information normally required in any of the foregoing techniques as well as some additional information. Initially the soil medium must be divided into similar strata. Boundary conditions must also be evaluated and locations for constant head and no flow or impervious boundaries identified.

The hydraulic conductivity of the soil medium is presumed to be characterized stochastically by a log normal probability density function (PDF). An estimate of the mean and standard deviation of the hydraulic conductivity must be determined. The method of estimating these values is to compile all the data available and assign weighting factors for the various sources and test procedures used. Perhaps more weight will be given to insitu tests than lab tests or correlations from other measured parameters. An estimate of the integral scale or distance over 
which the value of hydraulic conductivity is positively correlated must also be determined. This may be done by plotting the discrete values of hydraulic conductivity from samples taken in close proximity vertically and laterally, computing correlation coefficients and the resulting integral scale. (10)

Other data required are median grain size, the ratio of lateral to longitudinal microscopic or particle scale dispersivity and soil porosity. The median grain size may be determined from grain size distribution curves. Soil porosity may be estimated from standard penetration tests and soil descriptions. Figure 1 presents a correlation between standard penetration test results and porosity for different soils types. This figure is adapted from Navfac DM 7 (8). On the right ordinate scale the results of information were superimposed correlating density descriptions with standard penetration results from Peck (9).

The methodology to be described herein and presented in detail in the companion paper (2) can best be illustrated by example. The following two examples will demonstrate its use in solving real flow problems.

FLOW BENEATH A DIKE EXAMPLE

The State of Rhode Island is presently planning the construction of the Big River Reservoir in central Rhode Island. The reservoir will inundate approximately 7 square miles of land in two towns, Coventry and West Greenwich. Figure 2 presents a plan of the general area of the site. 


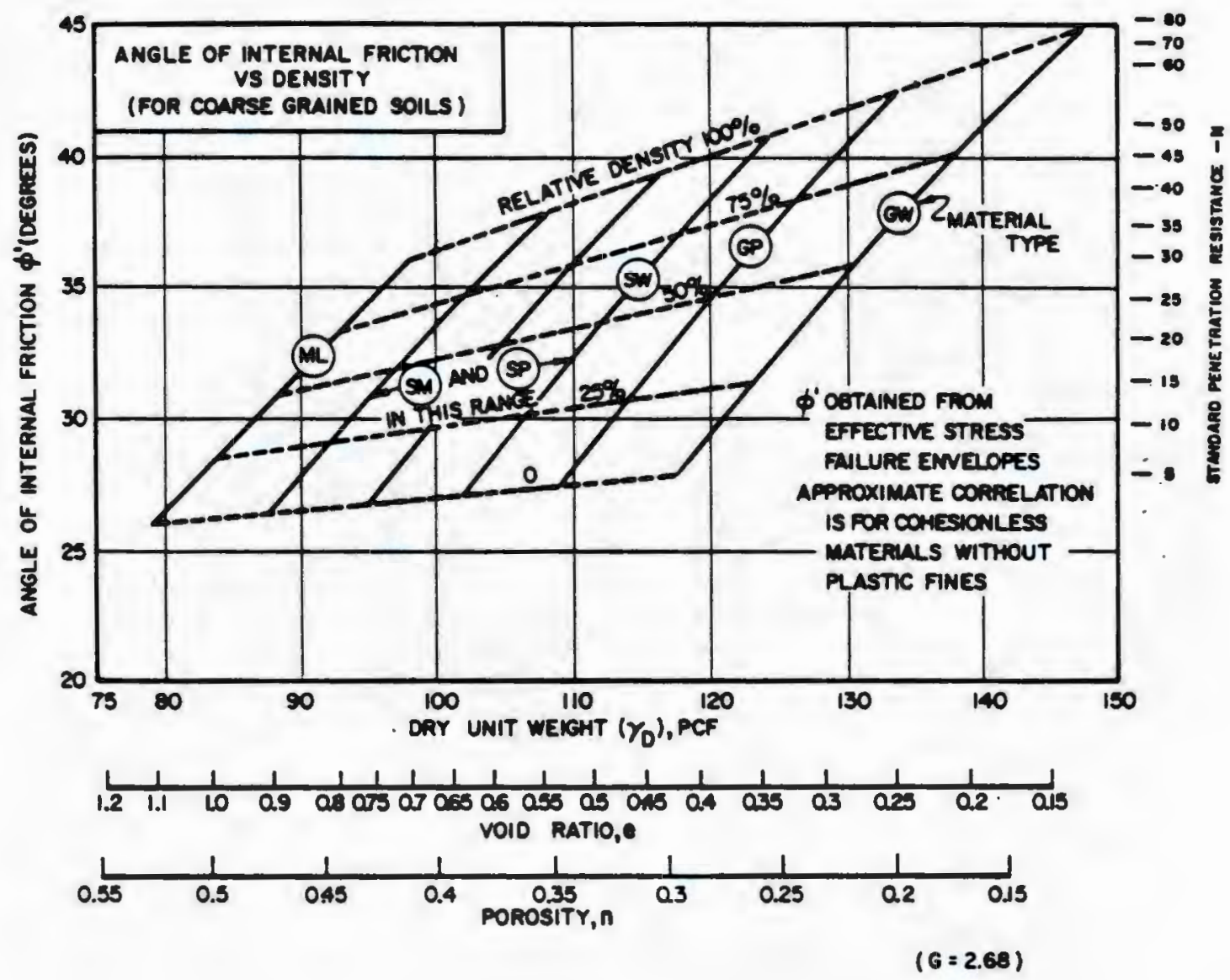

Figure 1 - Relationship Between Grain Size, Standard Penetration Test and Porosity, $(8,9)$ 


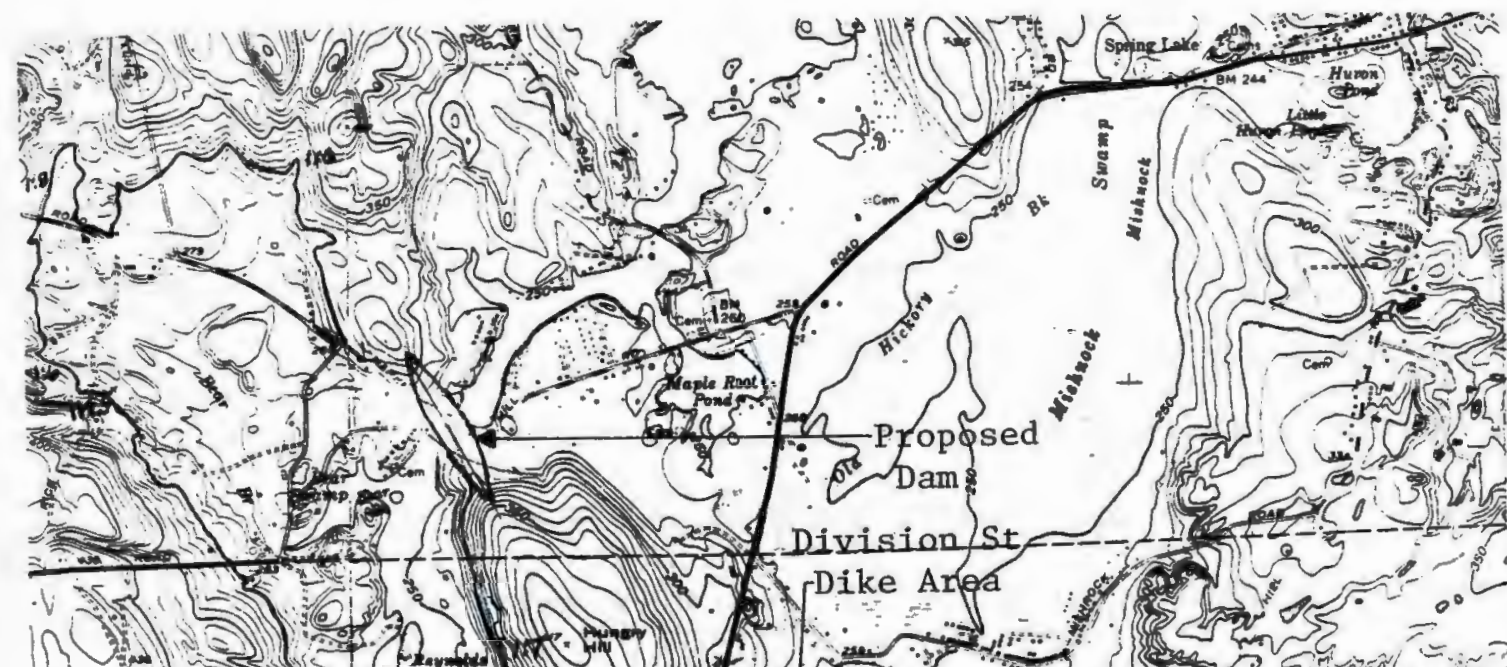

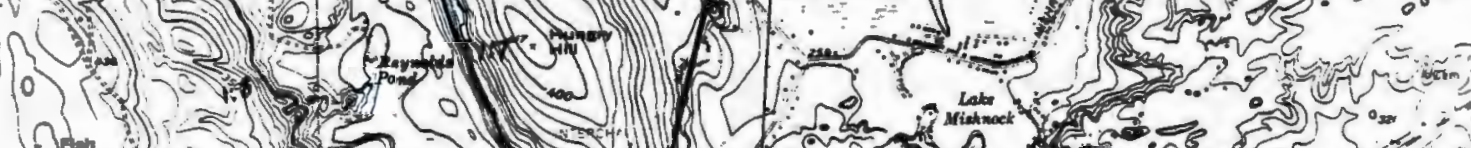
Un $=4$ (1)

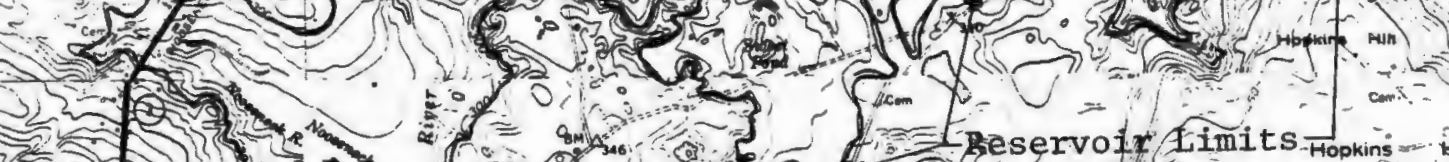
(3)

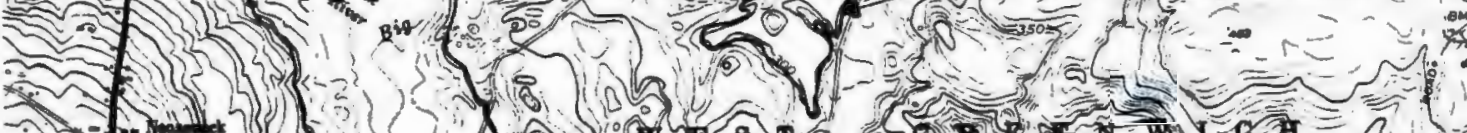

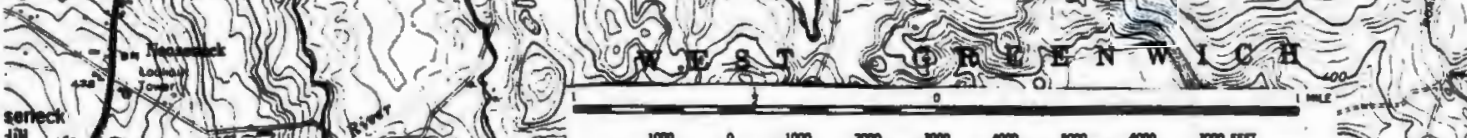
(2)

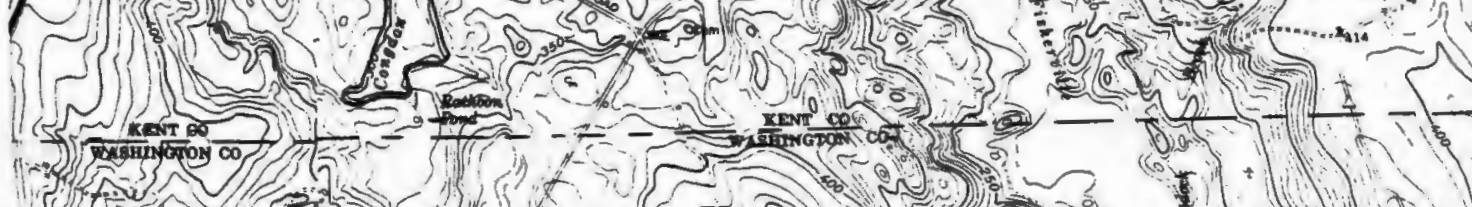

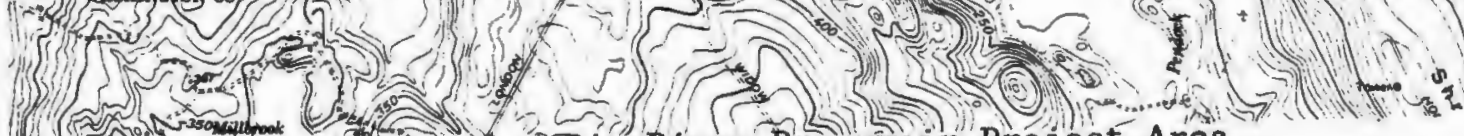
Figure 2 - Big River Reservoir Project Area 
Interstate Route 95 traverses this area and will divide the impounded water resulting in a reservoir on both sides of the road. For the eastern pool, this interstate highway will essentially form the northern boundary of the reservoir and Division Street will be flooded. Figure 3 presents a plan of the area with the groundwater contours reported in the preliminary geotechnical investigation (7). A groundwater divide is indicated near the proposed alignment of dikes along the southern side of Route 95 near Division Street, effectively separating existing groundwater flow patterns between those flowing south westerly into Carr Pond from those flowing northerly into Lake Mishnock. At the present time, as a consequence of the groundwater divide, the hydraulic gradient of the groundwater from the proposed dike alignment near Division Street to Lake Mishnock is nearly flat and we would expect very little groundwater flowing northerly into Lake Mishnock from the Division Street area. Apparently, the major groundwater contributions to this lake are presently from the east and west.

The design reservoir pool elevation is at 300 feet ( 91.5 meters) NGVD (National Geodetic Vertical Datum). The ground elevation along Route 95 in this area is approximately 310 feet (94.5 meters) NGVD. The ground surface drops off rapidly to the north in the direction of Lake Mishnock where the ground and water elevation is approximately 250 feet (76.2 meters) NGVD.

Upon completion and filling of the proposed Big River Reservoir and if no mitigating measures are taken, a rather large hydraulic gradient between the reservoir to the south of Route 95 and Lake Mishnock would 


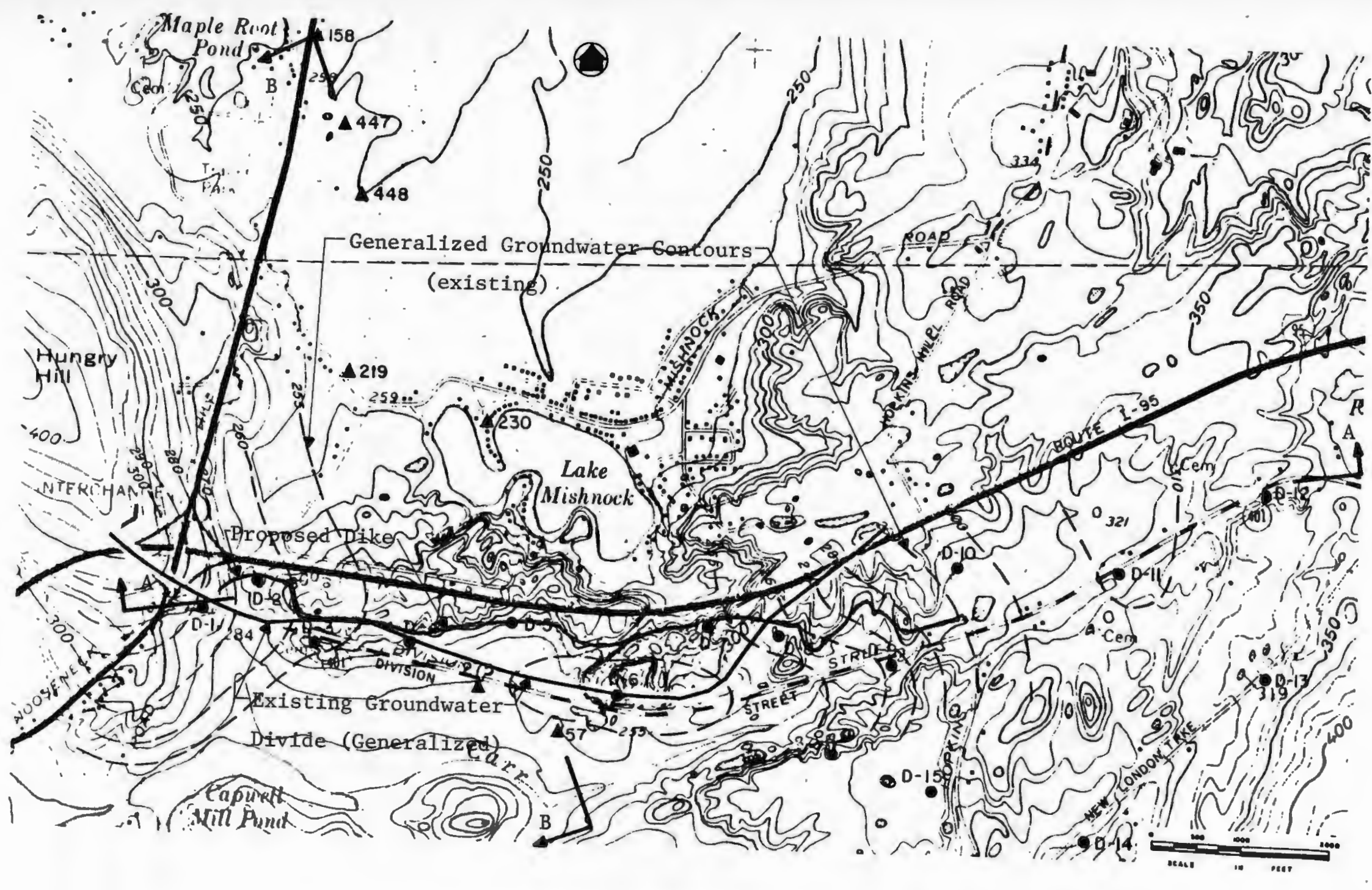

Figure 3 - Division Street Dike Vicinity Plan 
be set up. This would increase seepage into Lake Mishnock perhaps increasing water levels on a lake already ringed with homes only a few feet above water levels. Other homes between the reservoir and the lake might be similarly affected.

The site is underlain by relatively pervious glacial deposits consisting mostly of sand and silt. They are mapped as undifferentiated sand and gravel deposit and a kame terrace (10). The soils have clearly defined stratification which is visible in borrow cuts in the area but not easily recognized in boring samples. The uniform grain size and stratification suggests that it may have been deposited in a glacial lakebed with some thin coarser outwash deposits near the surface.

Figure 4 from KAME (7) presents a hydrogeologic section along the approximate alignment of the dikes. A deep valley was encountered in the area of boring D5 which coincides with the area of the steepest predicted hydraulic gradient under post construction conditions.

Figure 5 from KAME (7) presents a similar hydrogeologic section oriented perpendicular to that in Figure 4 and presents a section across the dike and along the location of potential leakage from the reservoir.

The major problem confronting the design engineer in this area is to quantify potential leakage from the reservoir if an impervious dike were constructed along the proposed alignment. Seepage losses beneath an impervious dike must be estimated with confidence. In addition, design alternatives to reduce leakage to acceptable levels need to be developed 


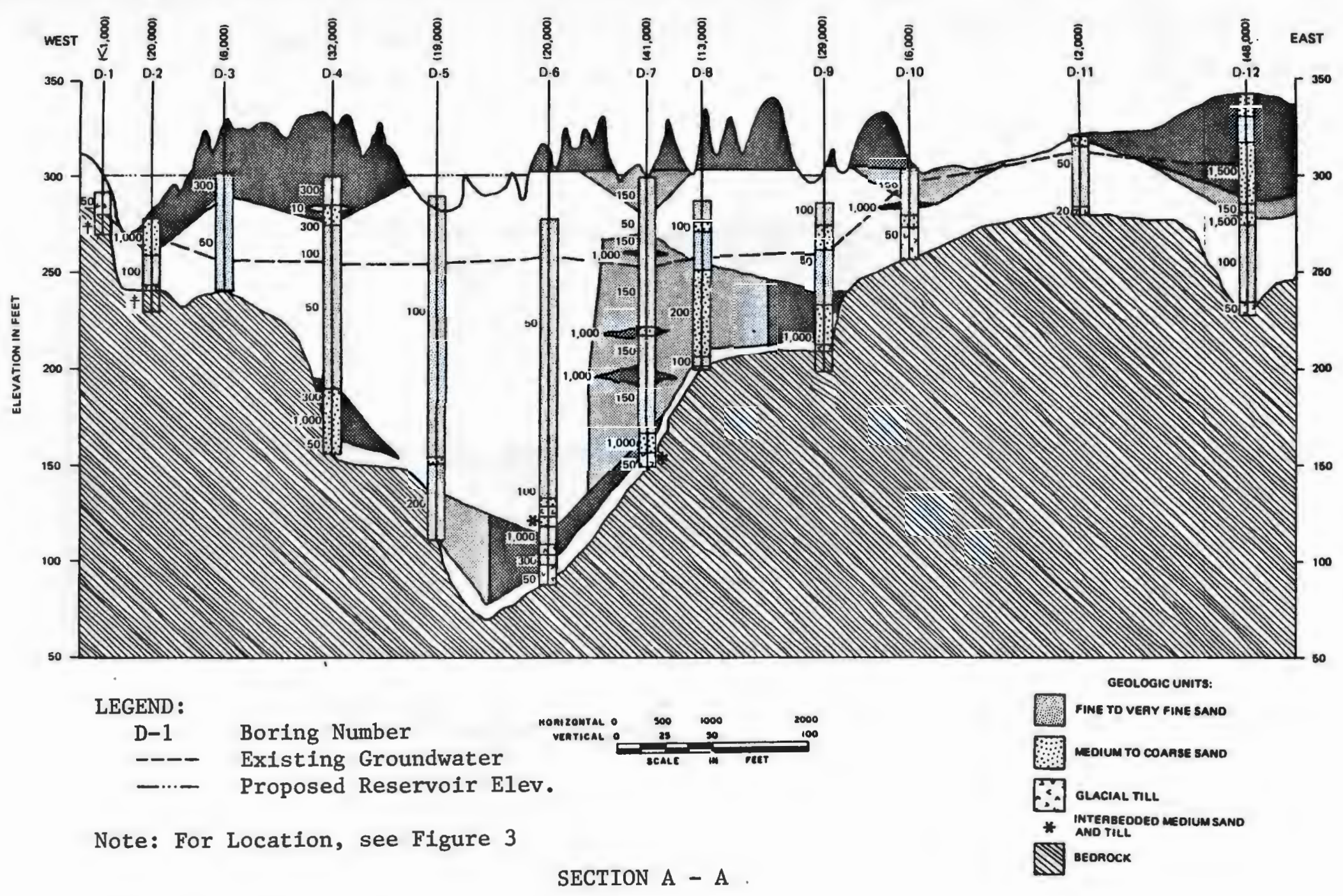

Figure 4 - Generalized Hydrogeologic Profile Number 1 


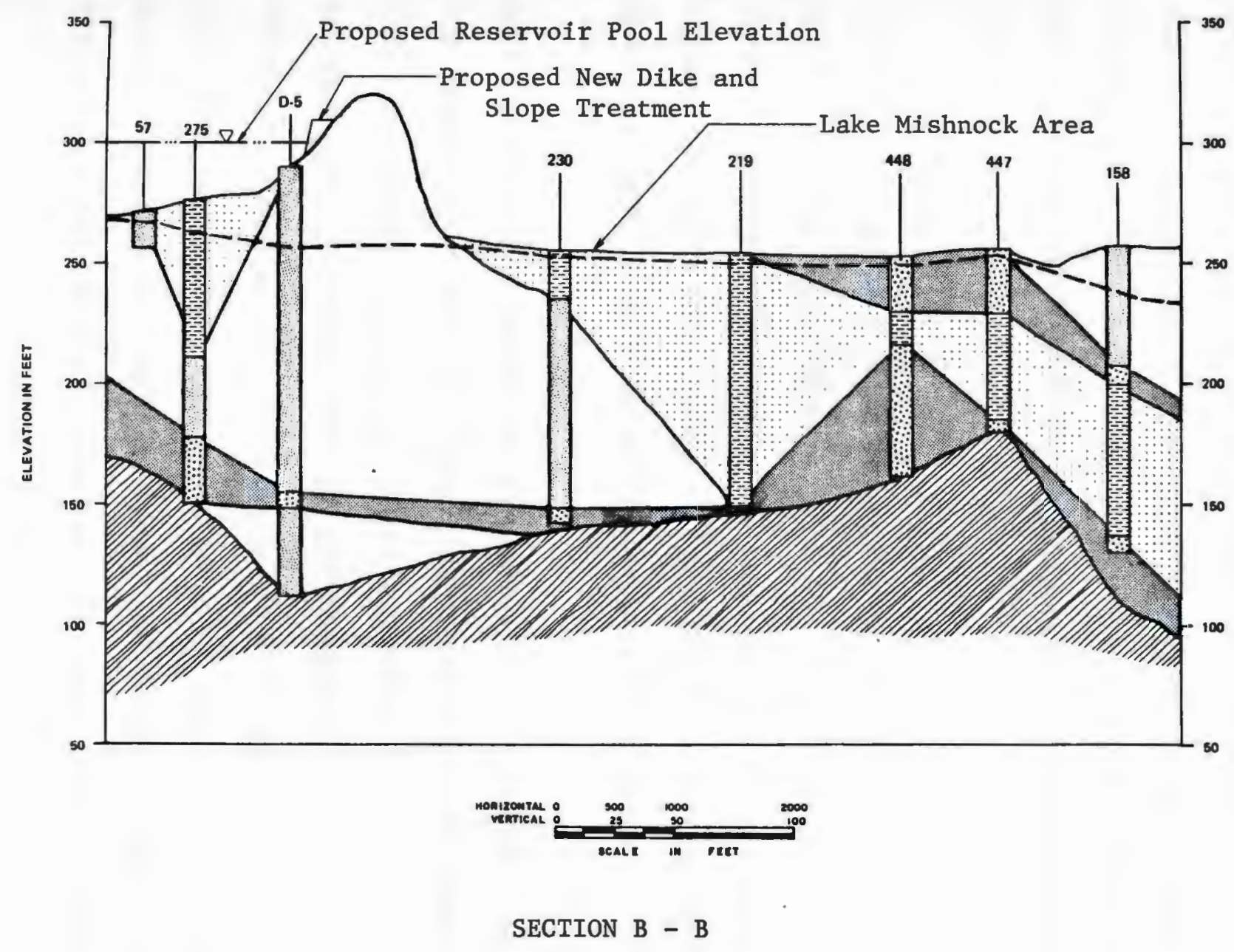

Clayey
Fine/v. Fine
Sand
Med/C Sand
Bedrock
v low hyd cond
low hyd cond.
med hyd cond.

Figure 5 - Generalized Hydrogeologic Profile Number 2 
and their effects on the seepage losses quantified with confidence.

An idealized soil profile assuming a singular stratum of soil was sufficient to characterize the soil profile was developed and is presented in Figure 6. The soil properties were estimated from the results of a series of laboratory tests. The mean and standard deviation of hydraulic conductivity and mean particle size were estimated from a series of grain size analyses on this soil. The autoregressive parameters were assumed equivalent to the results of field studies based on similar stratified soil by Smith (11). The porosity was estimated from soil descriptions and standard penetration test results.

A second soil profile which characterizes the soil conditions by using two soil strata is presented in Figure 7. The deeper soil stratum has a higher hydraulic conductivity and thus may act as a conduit for flow beneath the dike. Its thickness is limited to one row of the finite difference mesh located along the bottom of the section.

Both profiles have assumed an impervious barrier for the upper boundary for simplicity. The groundwater would actually flow upward somewhat after travelling beneath the impervious dike. In these examples, a stochastically generated mesh of autocorrelated hydraulic conductivity values are input to the numerical model while heads and resulting flow rate are determined. Subsequently mass transport is simulated by particles released from the upper left area of the region which eventually reach the right boundary. The main interest in this problem 

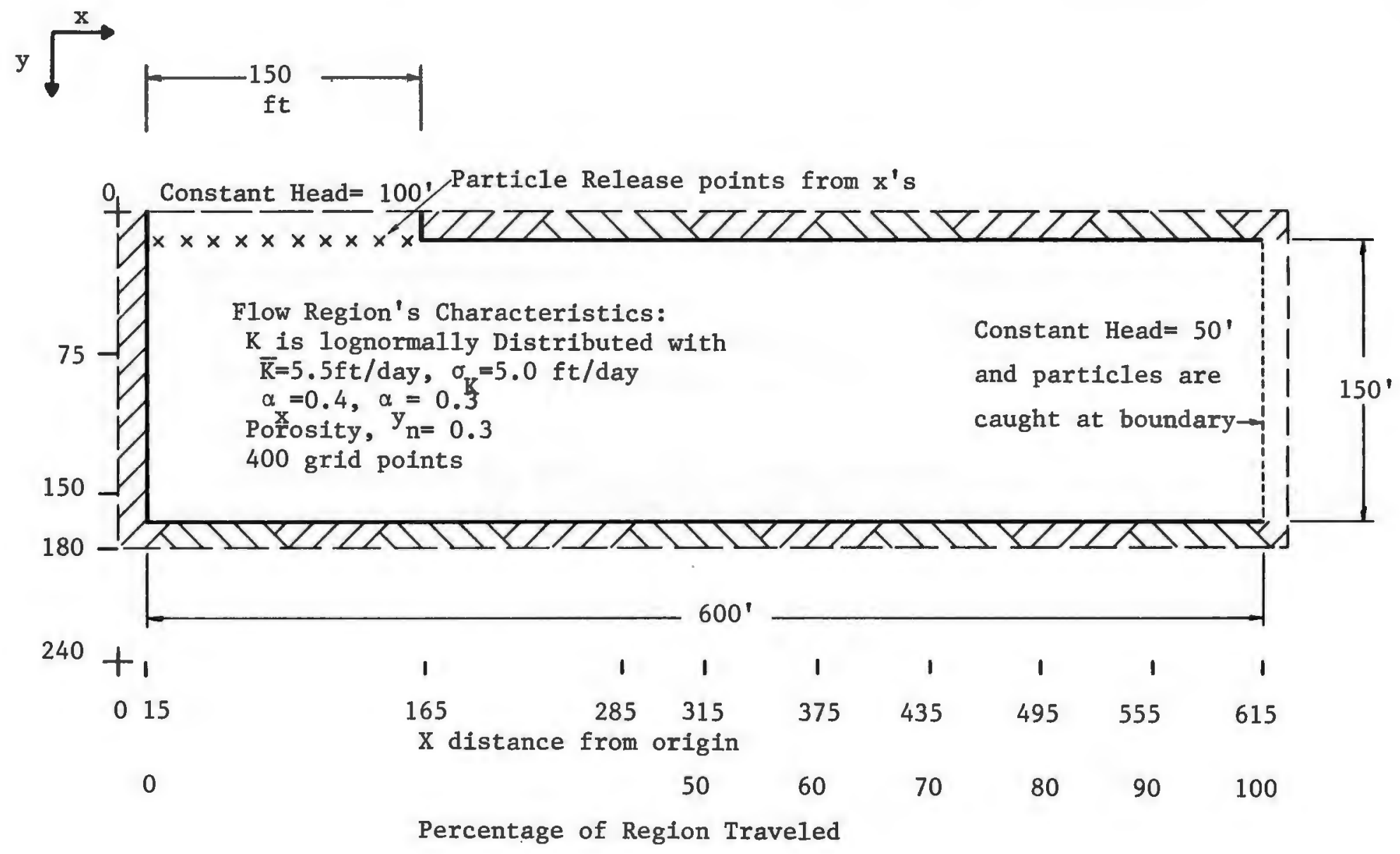
y<smiles>CC[AsH][AsH2]C</smiles>

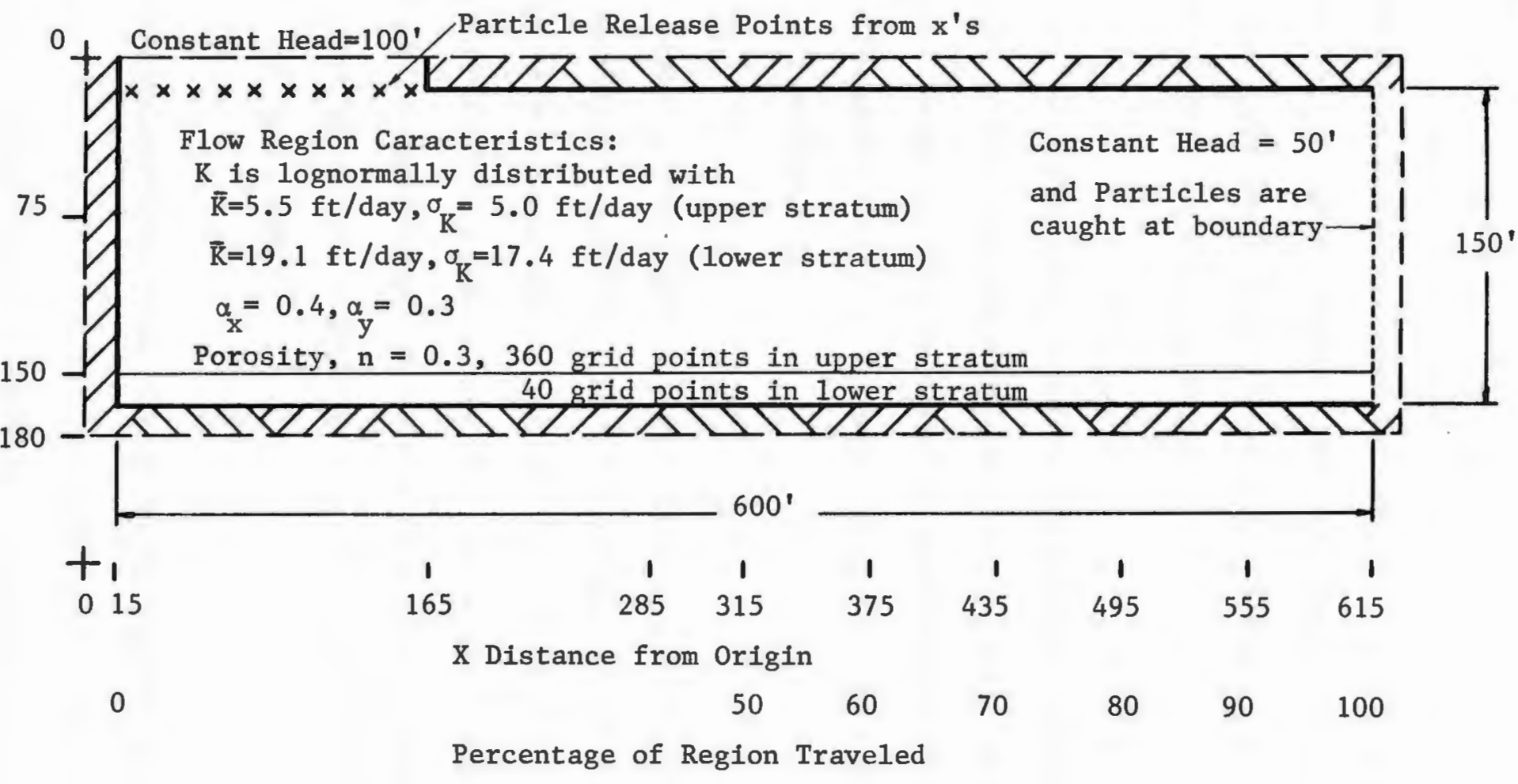


is the calculation of flow rate. Dispersion and travel times are of less concern.

The flow region and boundary conditions in this example do not result in uniform flow so a shape factor must be applied to the effective hydraulic conductivities. The technique to determine this shape factor was included in the companion paper (2).

The results of 40 Monte Carlo simulations for these two flow situations are summarized in Table 1. In addition to the two soil profiles already discussed is a third profile, profile $1 \mathrm{~A}$, which has the same layout but the soil is assumed to have a constant value for the hydraulic conductivity of 5.5 feet per day.

The intermediate coordinates during transit and travel time values listed are the mean values for all 400 particles which flow through the region. The reference to percentage of the region are as presented in Figure 6 and merely are a tabulation of time to get to that point in the region. Since an equal number of water particles are released from each of the 10 nodes which are release points, there is a bias in the travel times. More on this later.

These results demonstrate that the presence of the lower stratum of higher hydraulic conductivity increases flow by about 25 percent and lowers the mean value of the exiting $y$ coordinate by approximately twelve feet. The median y coordinate at the end dropped approximately 12 feet. For Profile 2, the median water particle exits within this 
$\underline{\text { TABLE } 1}$

SUMMARY OF RESULTS - DIKE EXAMPLE

Profile 1 Profile 1A Profile 2

Mean Values of Hyd. Conductivity (Ft/Day) Arithmetic

Geometric

5.52

4.10

5.50

5.50

7.21

Harmonic

Effective, Hydraulic

3.07

5.50

4.74

4.41

6.37

3.31

(After Applying Shape Factor)

3.81

5.50

Effective, Travel Time

3.26

5.04

(After Applying Shape Factor)

3.56

5.50

Std. Deviation of Hyd. Conductivity

5.00

5.43

4.99

Flow Quantity (Cubic Ft/Day)-Mean

Standard Deviation

56.5

3.4

81.6

69.6

Porosity

0.3

0.3

4.4

Mean Coordinates After 191 Days (x)

194.8

272.2

0.3

(y)

95.7

110.9

205.4

118.6

Mean y Coordinate at End

Median y Coordinate at End

122.2

122.3

135.3

131.5

$-$

151.2

Mean Travel Time (Days)

$50 \%$ Region

$60 \%$ Region

404.0

454.6

$70 \%$ Region

504.8

$80 \%$ Region

555.4

$90 \%$ Region

605.9

$100 \%$ Region

656.2

-

273.9

$-$

305.1

-

336.0

$-$

366.2

$-$

397.0

417.7

428.5

Std. Deviation of Travel Time (Days) $50 \%$ $60 \%$

$70 \%$

292.8

$80 \%$

292.2

$90 \%$

292.0

$100 \%$

292.3

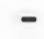

$-$

-

-

$-$

148.9
122.9

114.9

109.1

105.0

103.3

104.7 
lower stratum.

The adjusted mean values of the effective hydraulic conductivity fall within the expected range relative to the mean values calculated for the stochastic mesh of values for Profile 1 . The effective hydraulic conductivity values are fairly close to the geometric mean, although somewhat lower. The effective value determined by flow is larger than that determined by travel time. No similar comparison was possible for Profile 2 because of the different stratification.

Figure 8 presents a plot of the $x$ and $y$ locations for each water particle after 191 days. The upper plot is for the Profile 1 case, the lower plot for Profile 2. It is clear that the influence of the lower stratum in Profile 2 has caused the water particles to move downward while traversing toward the low head boundary at the right. Figure 9 presents a plot for the starting $x$ coordinate versus the ending $y$ locations for the two cases. This figure also demonstrates the skewing effect of the lower stratum in Profile 2.

Figure 10 presents the time to traverse the region versus the starting $x$ coordinate. Profile 1 results in a clear relationship of decreasing travel time with increasing starting $x$ coordinate. This should be anticipated since we would expect a higher velocity as well as a shorter route for those particles starting closest to the dike.

The flow rate for Profile 1 was computed as 56.5 cubic $\mathrm{ft} /$ day per linear foot with a standard deviation of 3.4 cubic ft/day. Figure 11 presents 


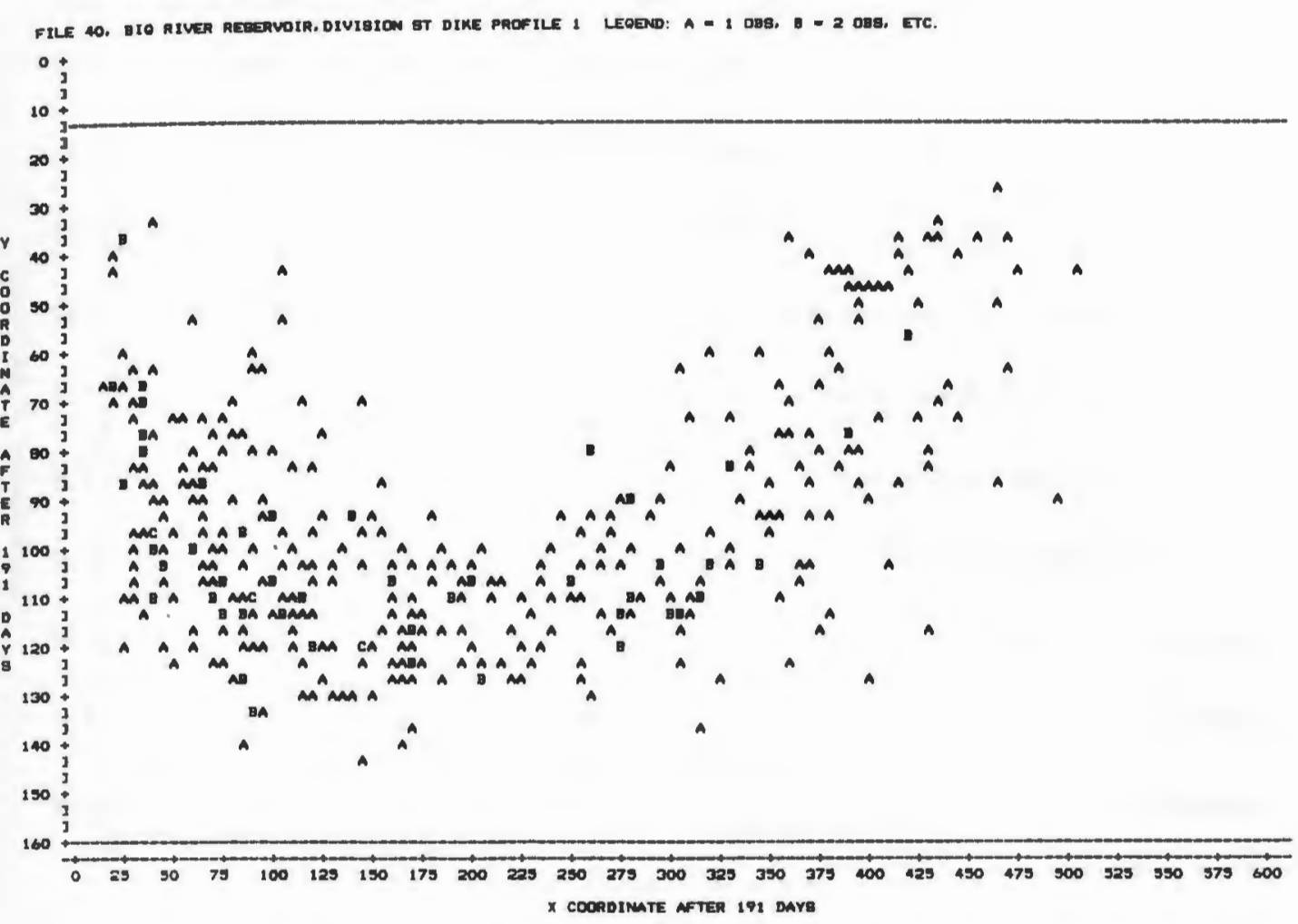

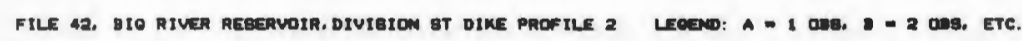

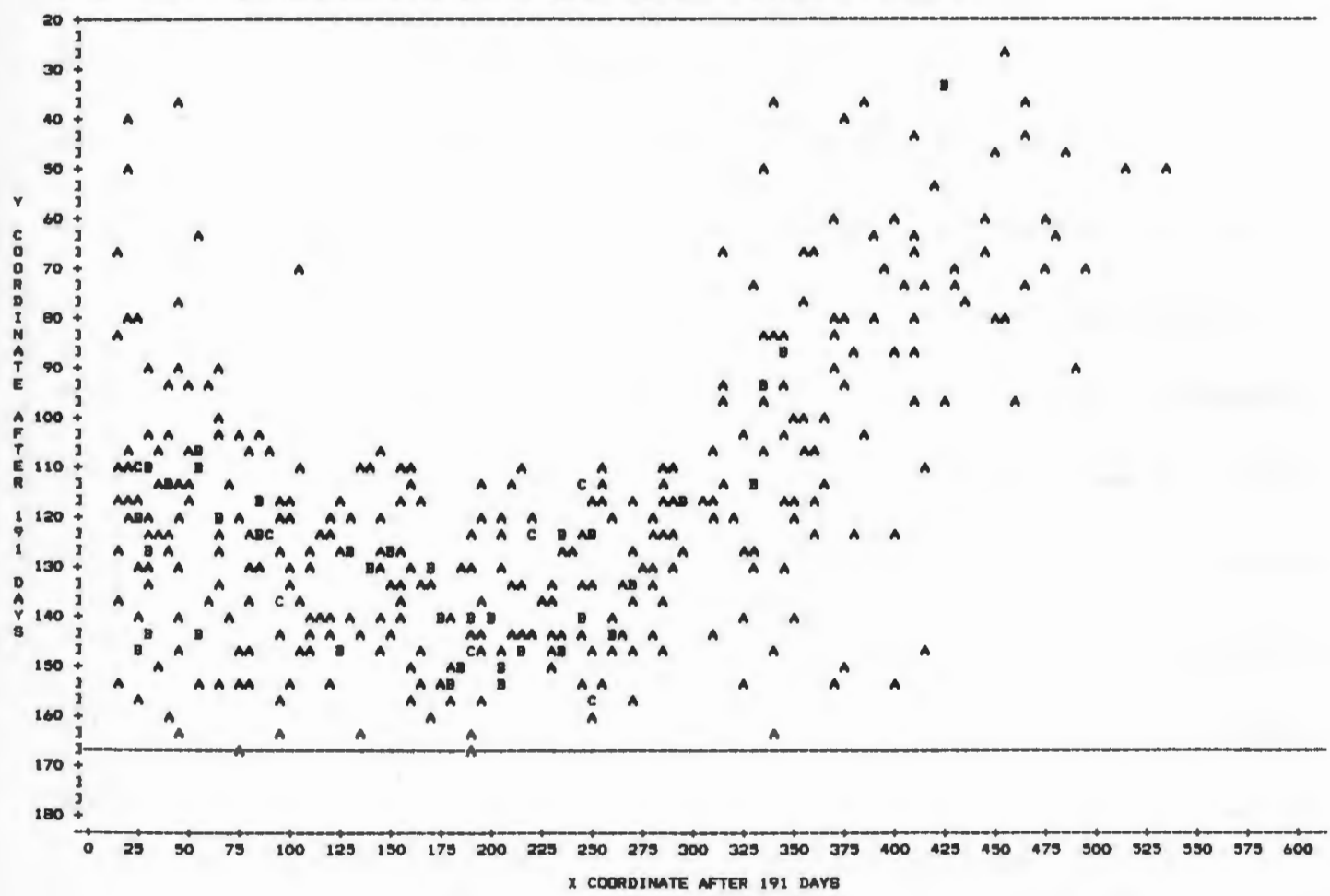

Figure 8 - X and $Y$ Coordinates of Particles After 191 Days 


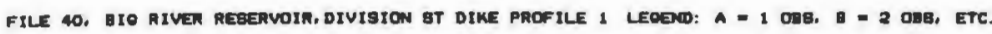

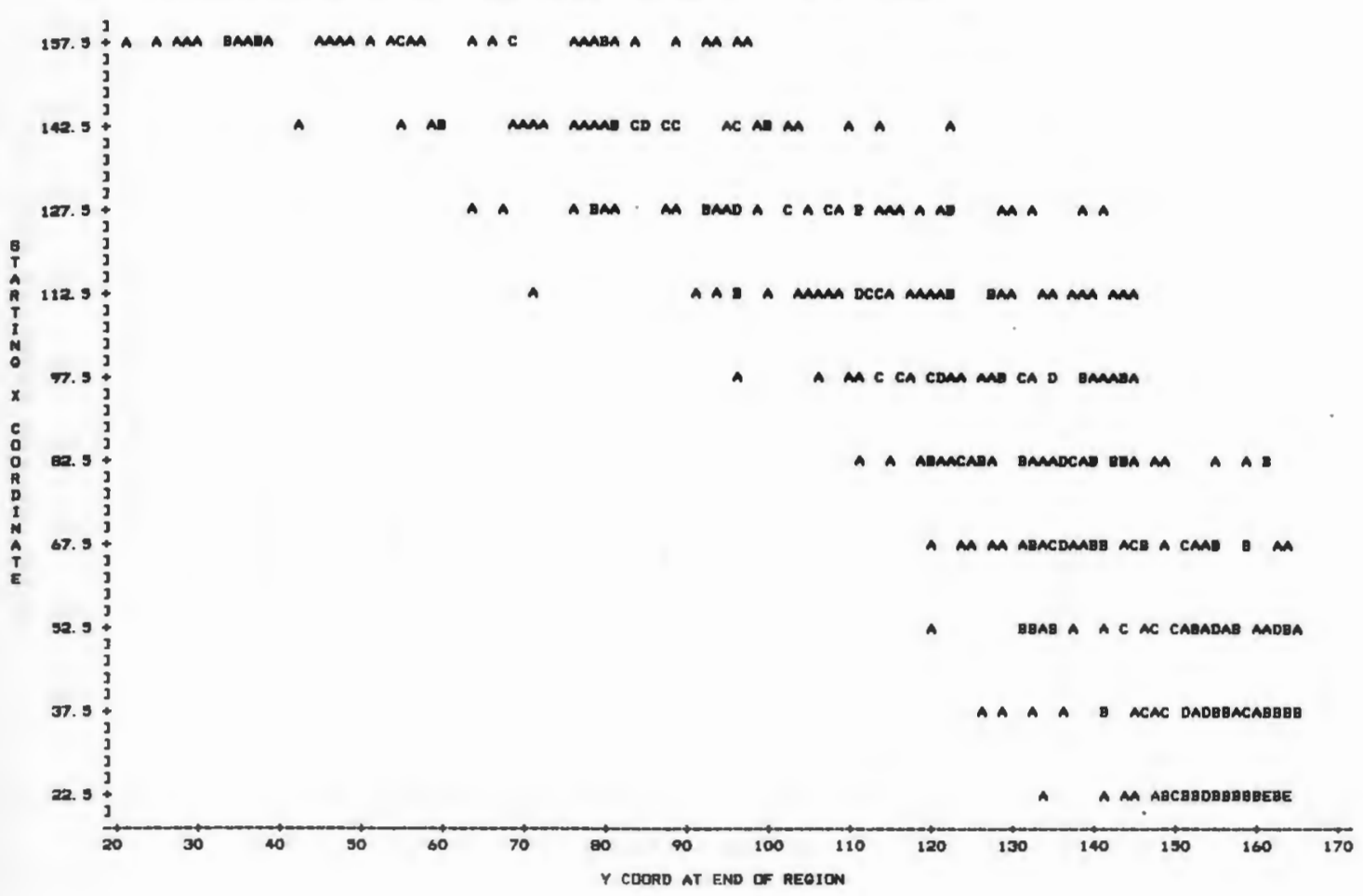

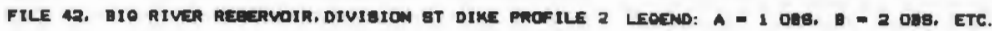

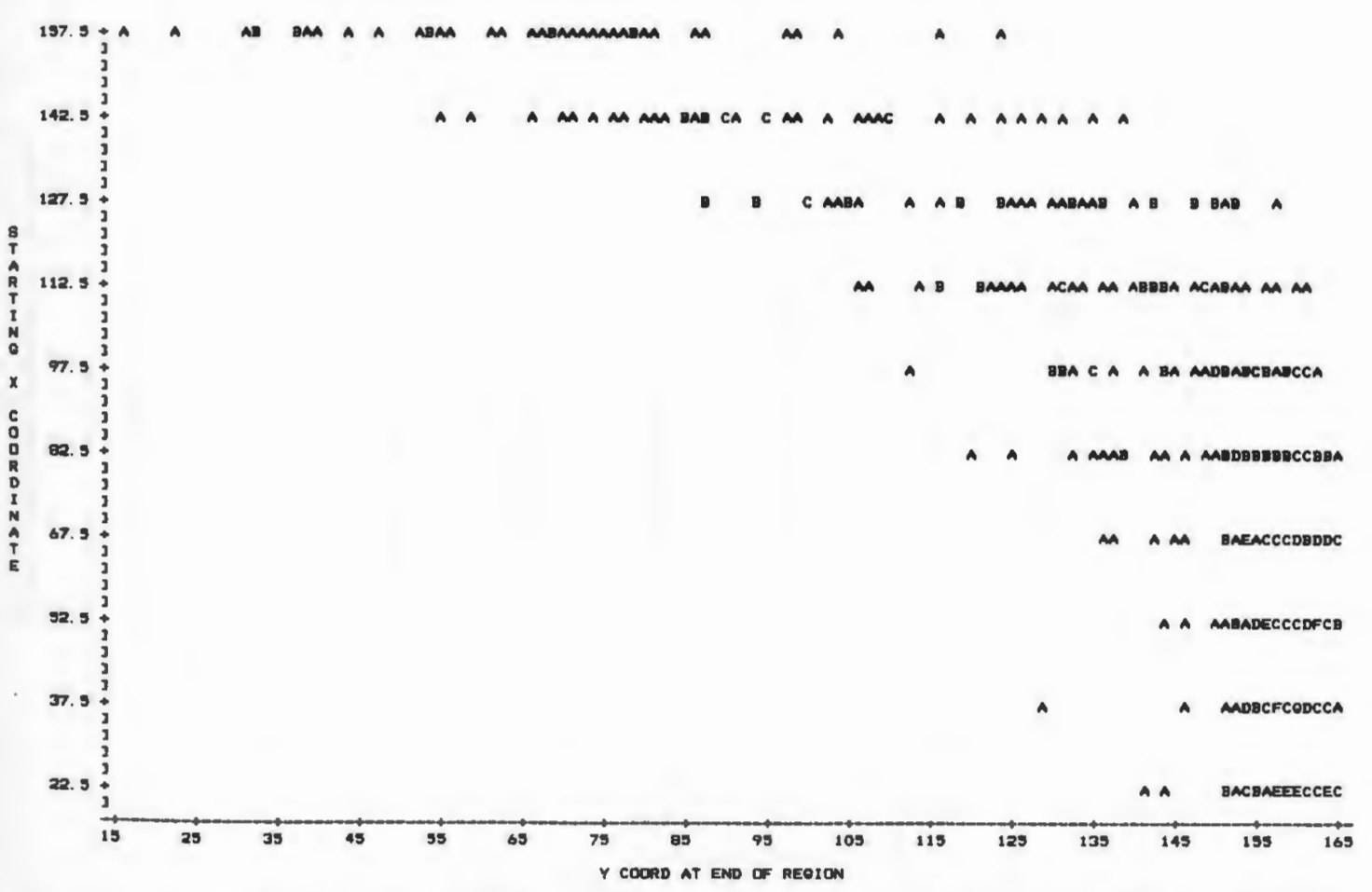

Figure 9 - Starting X Coordinate Versus Y Coordinate at End of Region 
FILE 40, BIO RIVER REgervatR. DIVISION gT DIKE PROFILE 1 LEOEND: A - 1 088. - 2 OBg, ETC.

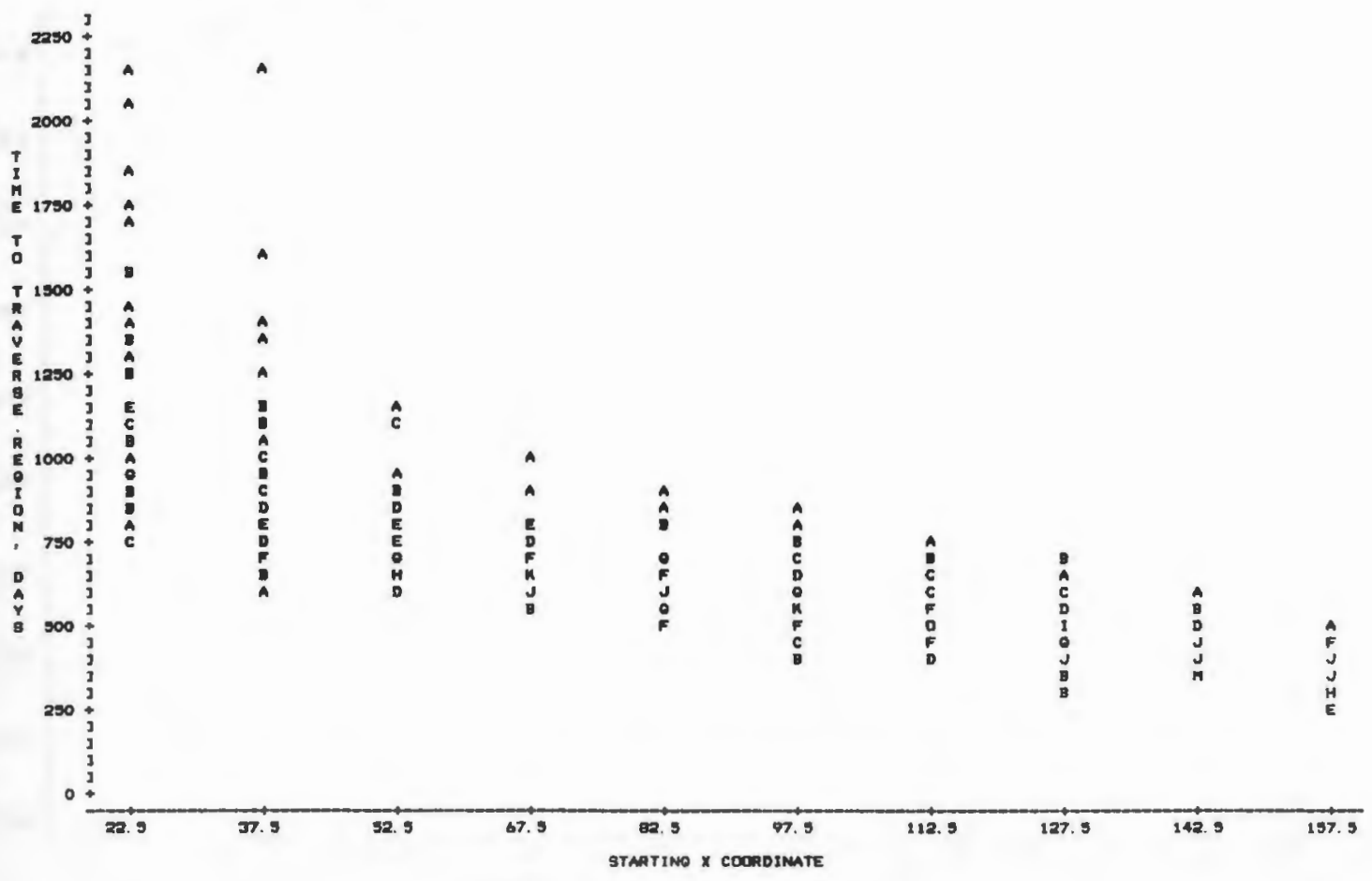

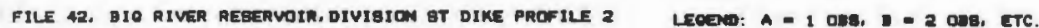

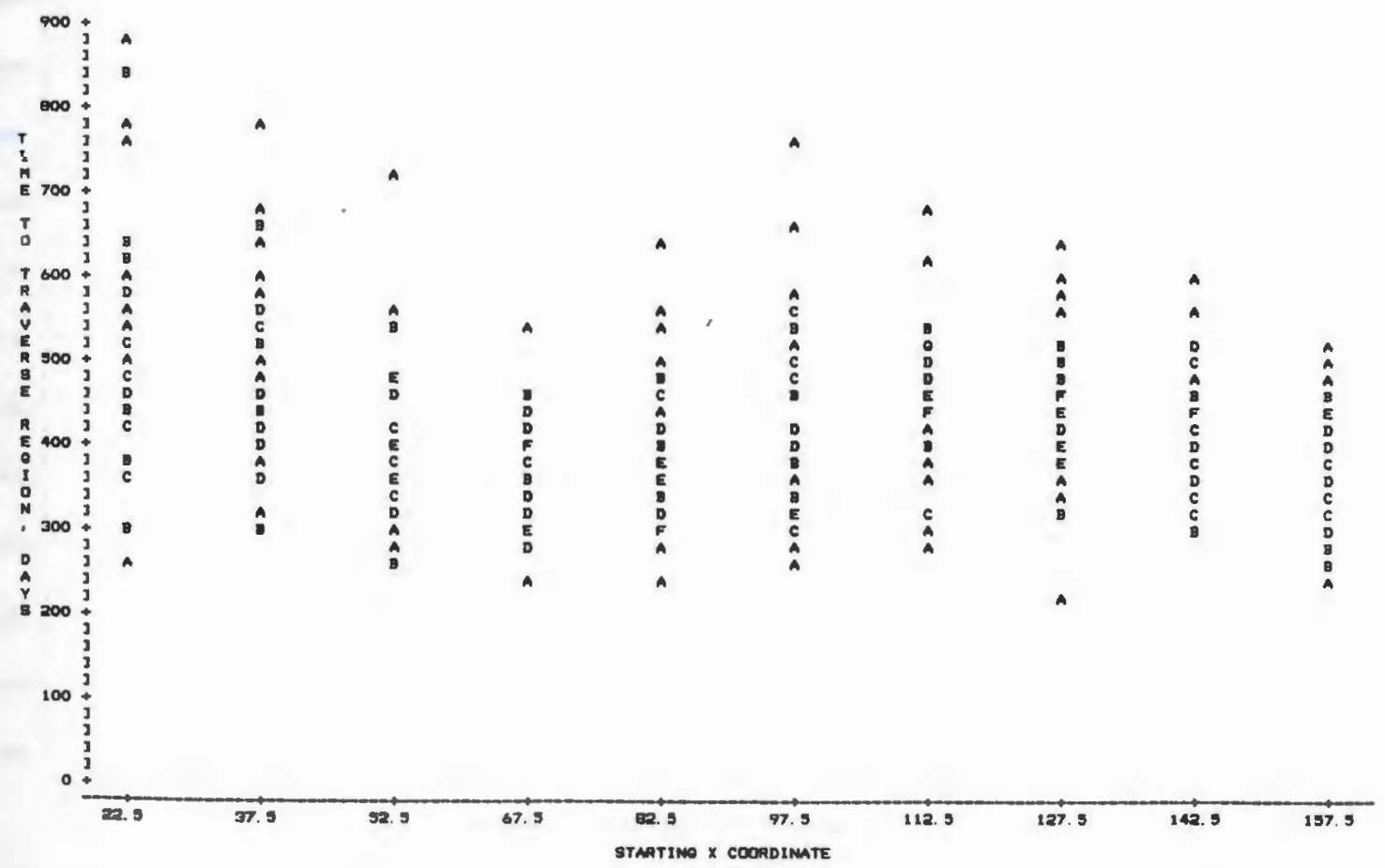

Figure 10 - Total Trave1 Time Versus Starting X Coordinate 
FTLE 40. TO ATVER MESERVOIA, DIVIGION GT DIKE PAOFILE 1
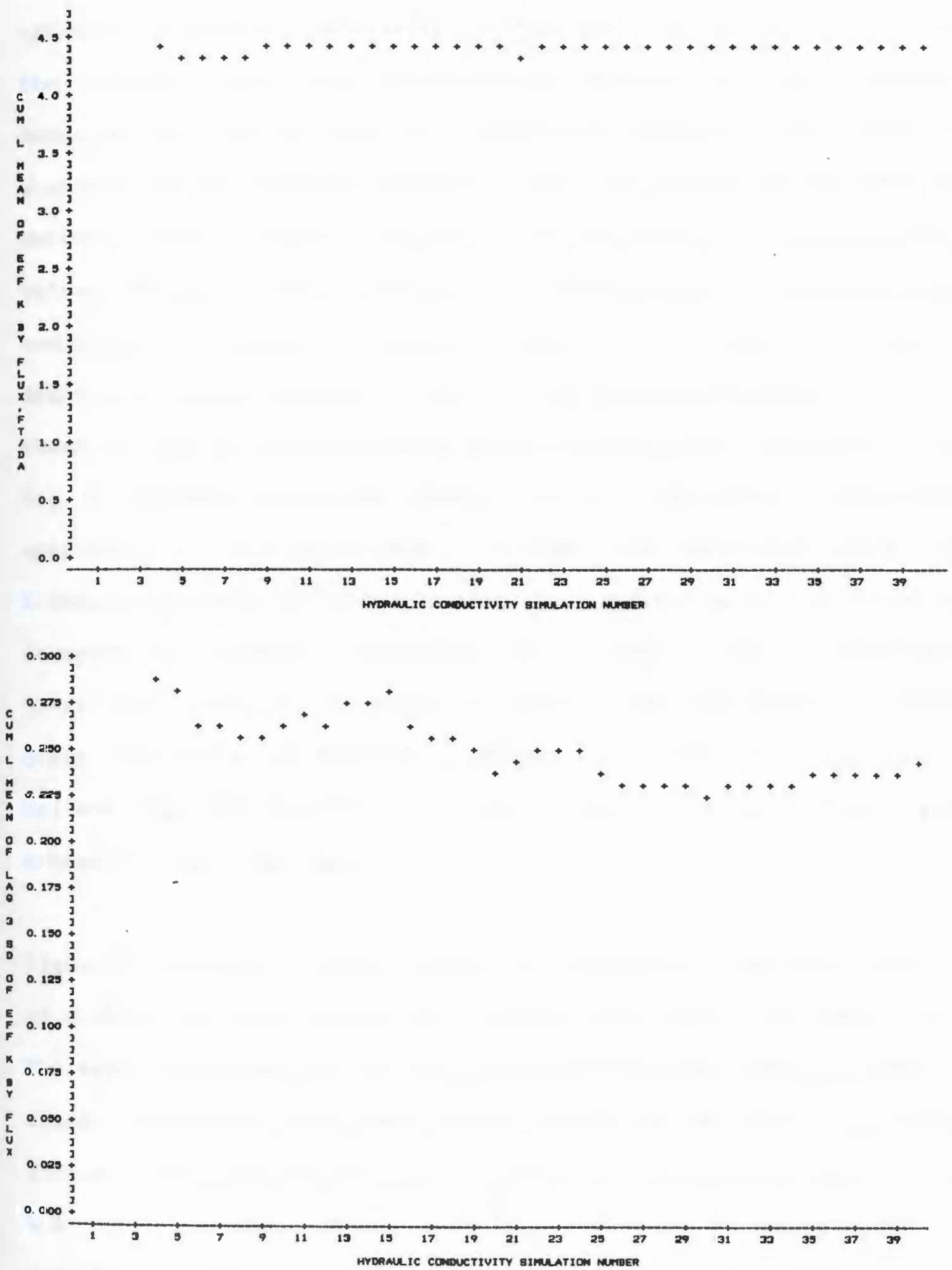

Figure 11 - Cumulative Mean of the Mean and Lag 3 Standard Deviation of Effective Hydraulic Conductivity Versus Simulation Number - Profile 1 
the cumulative mean of the mean and lag 3 standard deviation of the effective hydraulic conductivity for this profile. As was indicated in the companion paper (2), the cumulative mean of the lag 3 standard deviation may only be used as a qualitative measure of the trend and stability of the standard deviation. The true measure of the standard deviation would require calculation of that term for all preceeding values after each new observation is added. The effective hydraulic conductivity equals a constant times the flow rate. Figure 11 demonstrates that the mean value is very rapidly approached; in fact, there is very little fluctuation after 6 simulations. The mean of the lag 3 standard deviation reaches a fairly consistent level after approximately 26 simulations. A check for normality using the Kolmogorov D statistic indicates that the distribution of flow cannot be accepted as normally distributed to a high enough significance. Confidence limits can therefore not be predicted with accuracy. Even a quick observation of Figure 11, however, would certainly lead one to believe that the mean value of 56.5 cubic $\mathrm{ft} / \mathrm{day}$ is a fairly good estimate of the flow rate.

Figure 12 presents similar results for Profile 2. The mean value of $69.6 \mathrm{ft} /$ day is also reached very rapidly after only a few simulations. The value of the mean of the lag 3 standard deviation does not appear to reach a consistent mean value except perhaps in the last 4 or 5 simulations. The standard deviation of the flow rate was calculated to be 4.4 cubic ft./day. Again, normality could not be accepted by the calculation of Kolmogorov D statistic but the figure clearly indicates a 
FILE 42, ITO RIVER REgervoIh, DIUIBION GT DIUE PRUFILE

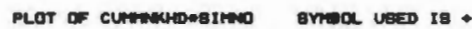
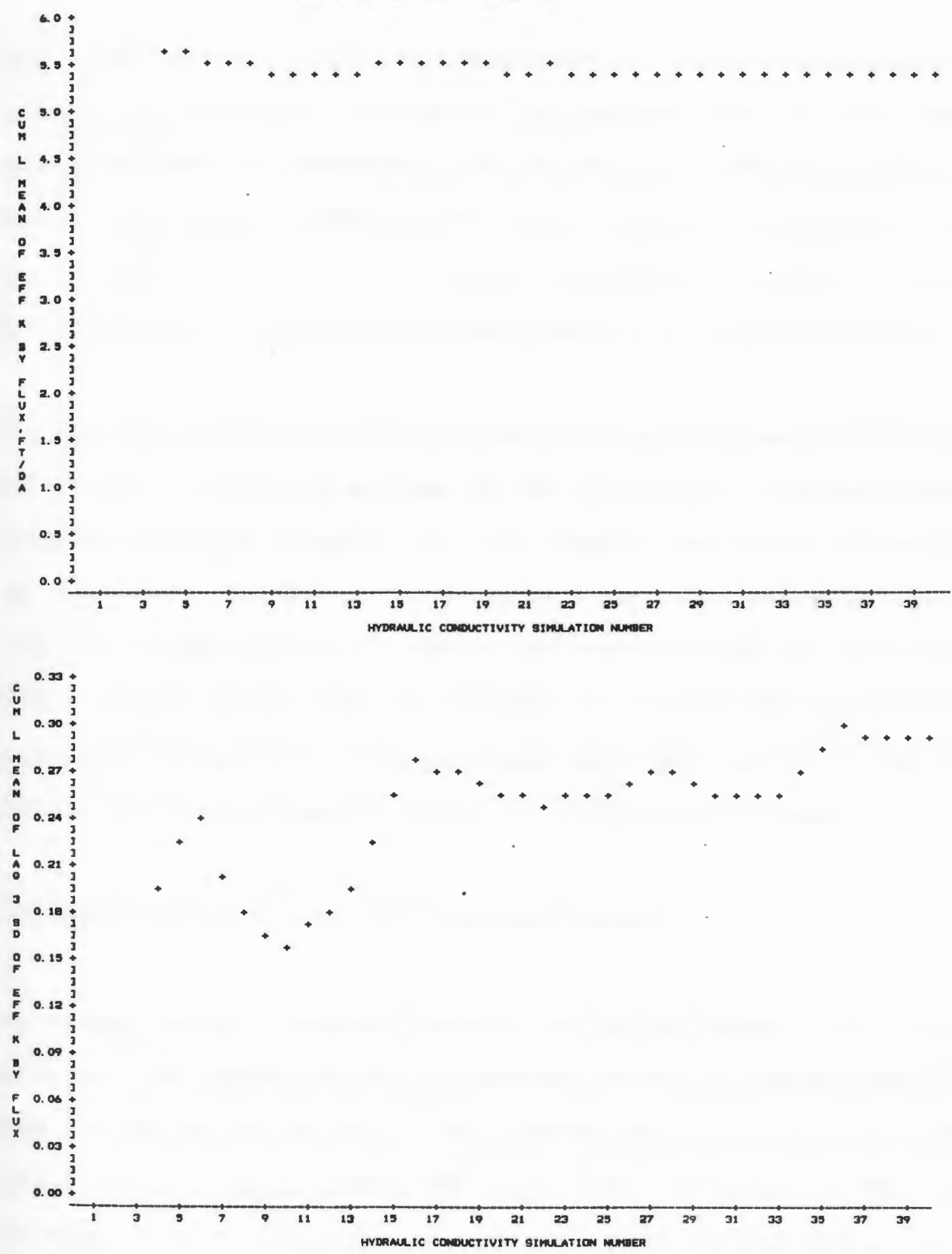

Figure 12 - Cumulative Mean of the Mean and Lag 3 Standard Deviation of Effective Hydraulic Conductivity Versus Simulation Number - Profile 2 
consistent trend in predicted flow rates.

This analysis would assist the geotechnical engineer in making predictions of groundwater flow beneath the proposed dike. It would also demonstrate that the determination of the presence of the high hydraulic conductivity stratum overlying the bedrock would be an important one. In any case, this flow rate is likely to produce an increase in the groundwater levels around Lake Mishnock which is an unacceptable result.

The next step in the analysis which could be completed would be to study the effect of mitigating measures on the flow rate. A seepage blanket could be simulated extending the left boundary and thereby increasing the flow path. An alternative would be a partially pentrating cutoff wall to increase the travel path of the water downward and around the wall. Similar results would then provide the geotechnical engineer with sufficient information on the predicted flow rates and variations so that the decision on which alternatives to utilize may be made.

GROUNDWATER IN THE VICINITY OF A LANDFILL EXAMPLE

The second example considered was for flow in the vicinity of a waste landfill. The example is for a hypothetical landfill although landfills with similar conditions exist. This landfill has been sited upgradient from a waterbody approximately 600 linear feet (183 meters) as depicted in Figure 13. The landfill was initially begun by excavating to or somewhat below the water table to allow excavation of cover material. The landfill materials were then dumped in the excavation and this 

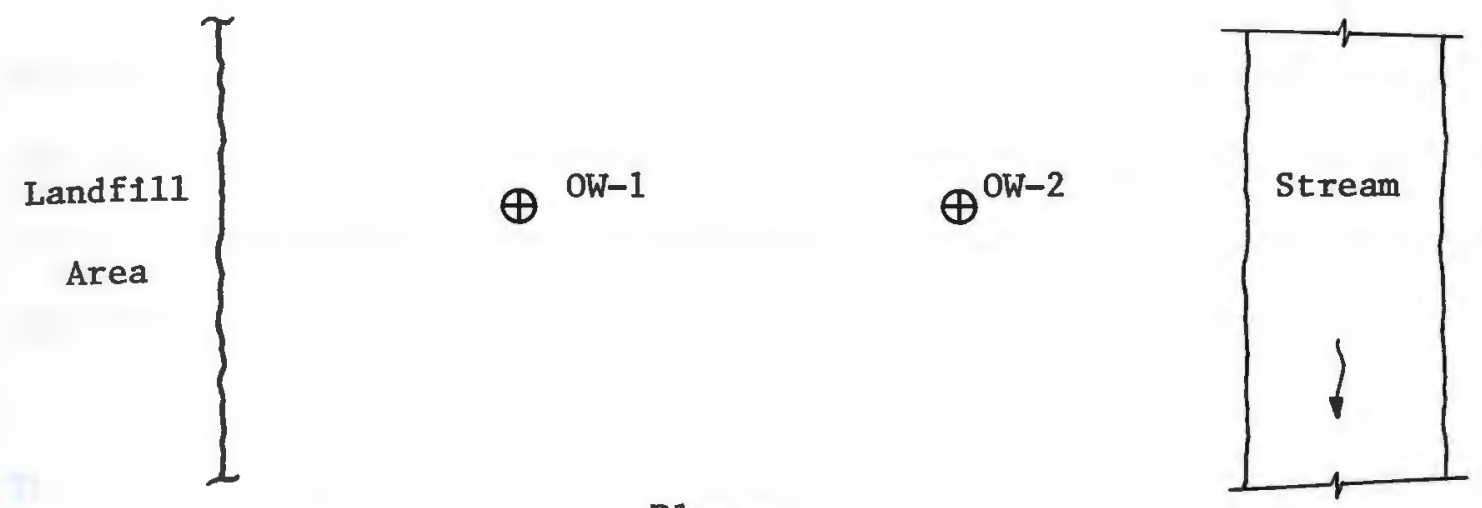

- P1an -

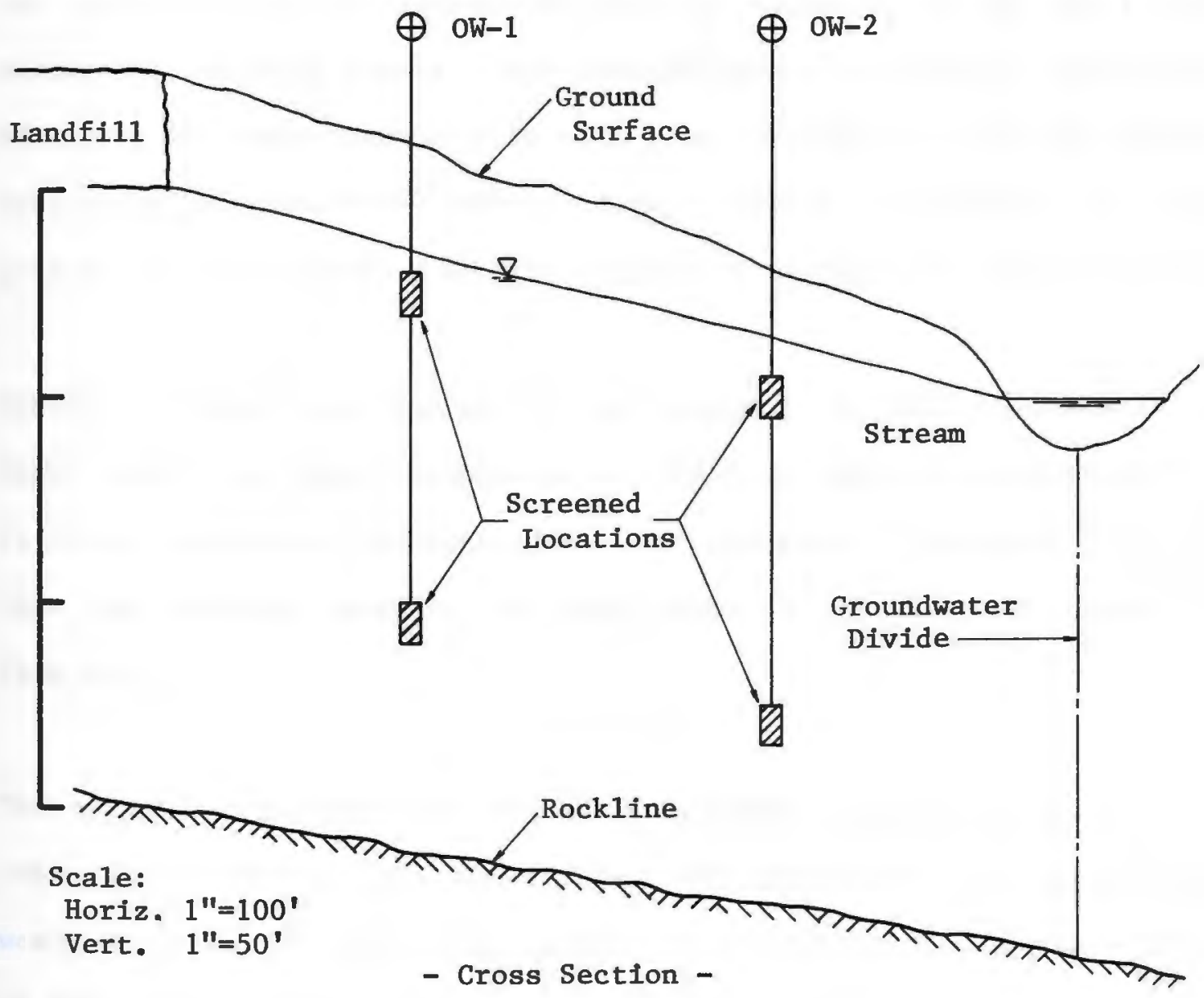

Figure 13 - Landfill Vicinity Plan 
dumping has continued for several years.

Monitor wells down gradient from the landfill were installed recently and have indicated some contamination. An investigation into the extent of this contamination, the time required for this contamination to reach the stream, as well as the estimated flow quantity is desired.

The soil profile consists of a stratified fine sand and silt with the same properties as the earlier example. Its thickness is 150 feet ( 45.5 meters) overlying a crystalline bedrock. The upper and lower boundaries are considered no flow boundaries with the exception of the small area within the landfill itself. The left boundary is a constant head boundary and the right boundary is a no flow boundary beneath the stream indicating a groundwater divide there. Figure 14 presents the soil profile utilized by the computer program to simulate the flow problem.

Table 2 presents the results of the analysis of this simulation. A shape factor was again determined as before to adjust the values of the effective hydraulic conductivities. The adjusted values again indicate that the geometric mean is the best predictor of the travel time and flow rate.

The mean flow quantity for the 40 simulations was calculated at 43.2 cubic ft/day flowing into the stream. The quantity of leachate present would be a part of this total quantity determined by the characteristic of the leachate generation process at the landfill. Assuming no change in the hydraulic head at this point, the results of the travel time 


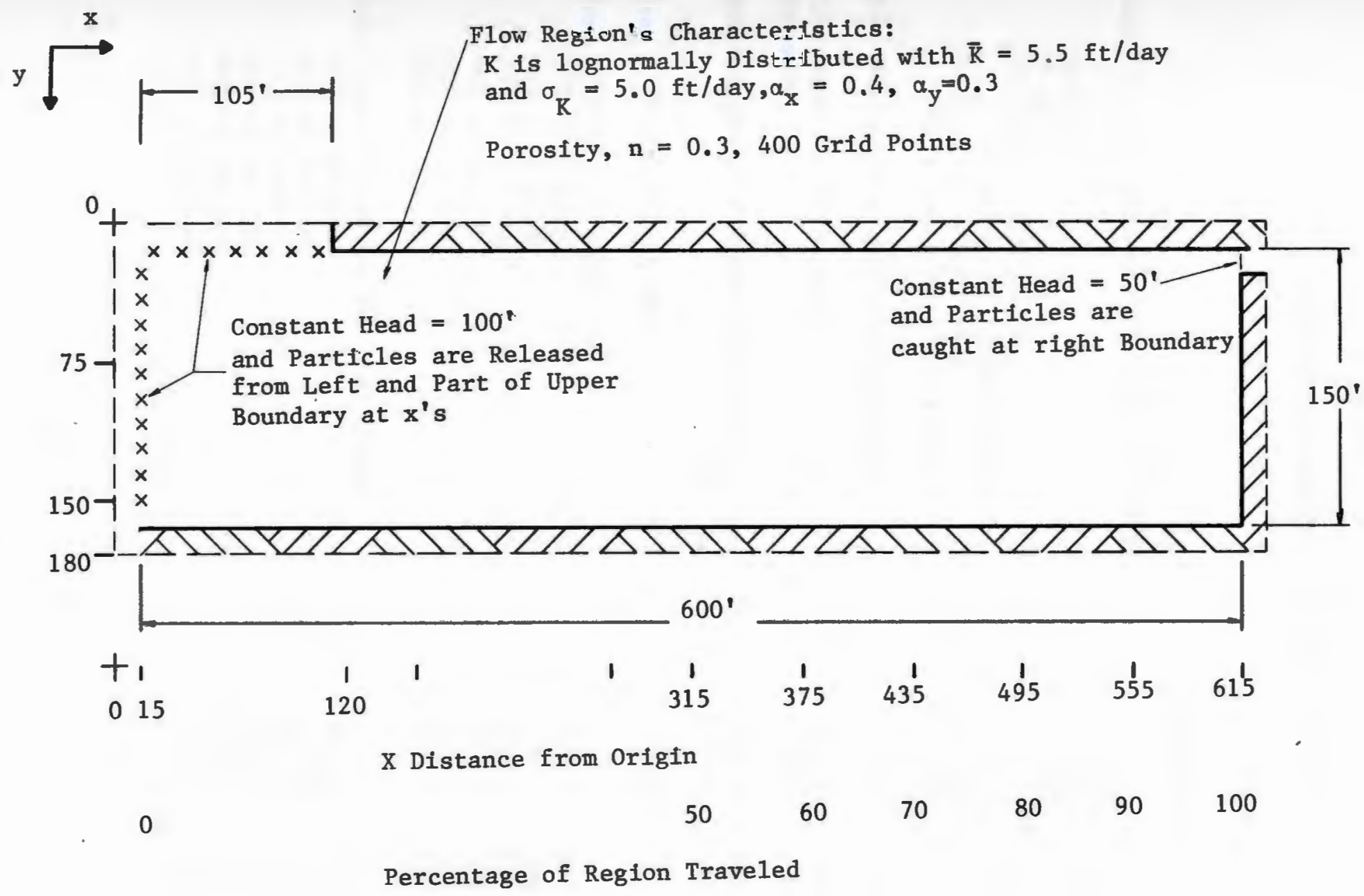

Figure 14 - Computer Simulated Landfill Profile 


\section{TABLE 2}

SUMMARY OF RESULTS - LANDFILL EXAMPLE

\section{Profile 1 Profile 1A}

Mean Value of Hyd. Conductivity (Ft/Day)

Arithmetic

Geometric

Harmonic

Effective, Hydraulic

(After Applying Shape Factor)

Effective, Travel Time

(After Applying Shape Factor)

Std. Deviation of Hyd. Conductivity

Flow Quantity (Ft3/day)

Porosity

Mean Coordinates After 191 Days (x)

(y)

Mean y Coordinate at End

Mean Travel Time (Days)

$50 \%$ of Region

$60 \%$ of Region

$70 \%$ of Region

$80 \%$ of Region

$90 \%$ of Region

$100 \%$ of Region

Std. Deviation of Travel Time (Days)

$50 \%$ of Region

$60 \%$ of Region

$70 \%$ of Region

$80 \%$ of Region

$90 \%$ of Region

$100 \%$ of Region
$5.46 \quad 5.50$

$4.05 \quad 5.50$

$3.01 \quad 5.50$

$3.44 \quad 5.07$

$3.73 \quad 5.50$

$3.15 \quad 4.89$

$3.54 \quad 5.50$

$4.87 \quad 0$

$43.2 \quad 64.4$

$0.3 \quad 0.3$

$172.5 \quad 250.7$

$\begin{array}{ll}65.6 & 77.2\end{array}$

$27.8 \quad 27.7$

$392.2 \quad 240.9$

$457.4 \quad 282.5$

$522.6 \quad 324.2$

$584.2 \quad 365.5$

$642.9 \quad 405.0$

$681.9 \quad 430.6$

$\begin{array}{ll}179.0 & 87.3 \\ 181.5 & 87.4 \\ 182.2 & 87.9 \\ 183.4 & 89.1 \\ 190.1 & 92.9 \\ 199.5 & 97.6\end{array}$


simulations indicate that approximately $45 \%$ of the water exiting at the river will pass through the landfill and be contaminated.

The mean $x$ and $y$ coordinates after 191 days are 218.8 feet and 54.5 feet, respectively. Figure 15 presents a plot of the $x$ and $y$ coordinates of the flow particles at this time was well as a plot of the starting $x$ coordinate versus the $y$ coordinate at the end of the region. This figure illustrates that the particle swarm separates somewhat with a large number of the particles moving downward into the flow region, the lowest penetration being 120 feet into the 150 foot thick sediment. Generally those particles starting from the end of the landfill closest to the surface water body stay near the surface and exit the highest. Those which start from the upgradient side plunge deeper and exit lower into the stream.

Figure 16 presents the time to traverse the region versus the starting $x$ coordinate. A relationship is suggested similar to that in the dike example that the travel time is increased as the distance away from the river increases. This is expected and is undoubtedly due to the lower velocity, the longer travel distance and the indirect route taken downward into the sediment by those particles farthest from the stream.

The distribution of travel times could not be accepted as normally distributed by the Kolmogorov D statistic because of the several extremely large travel times recorded. Consequently, confidence limits on the travel time could not be developed. However, by inspecting Figure 17 which presents a histogram and breakthrough curve for this 
FILE 45, FLON FROM LANOFILL EXATPL

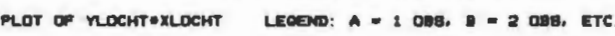
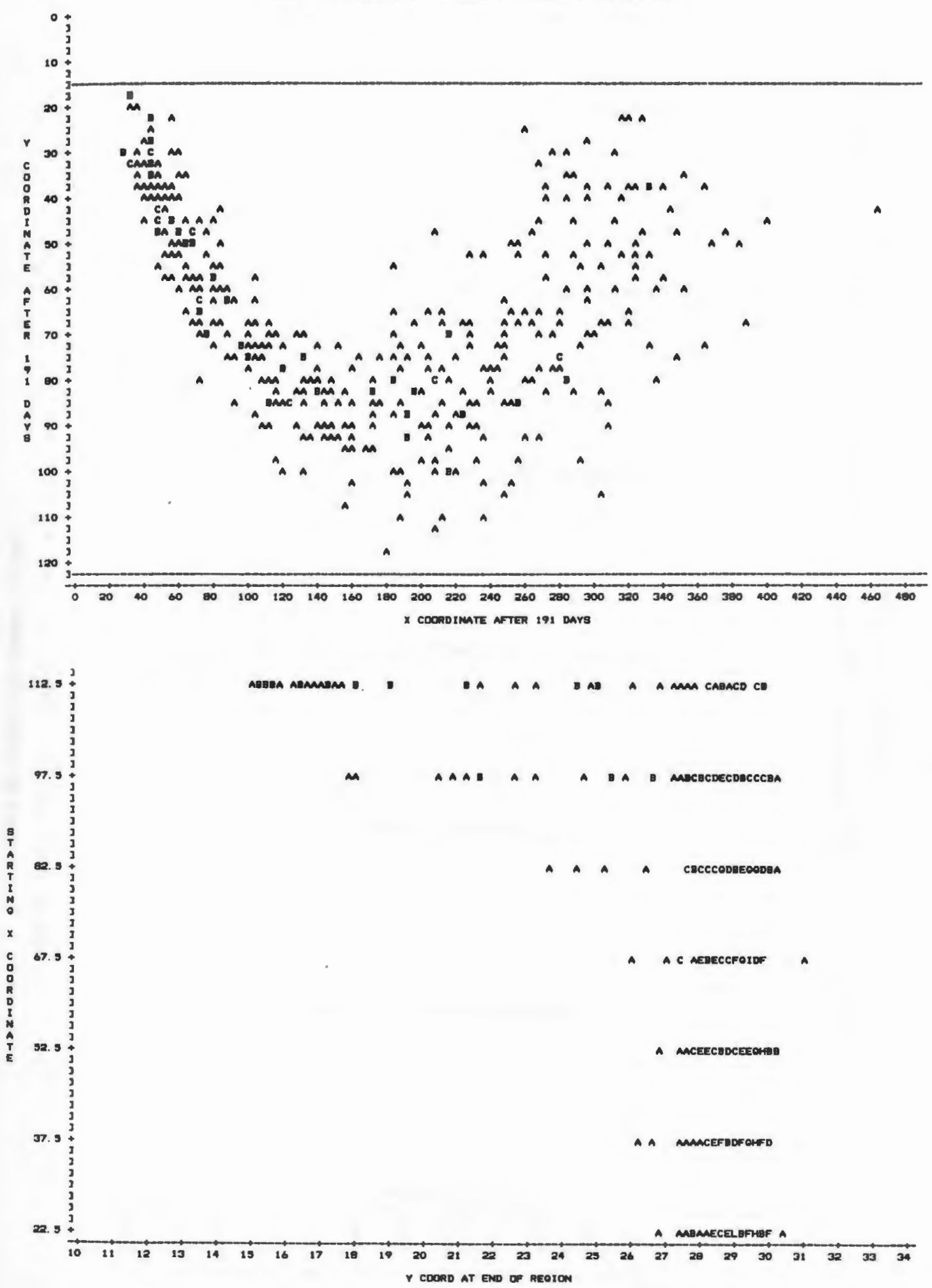

Figure 15 - Particle Location after 191 Days and Starting X Coordinate Versus Ending $Y$ Coordinate 
FILE 45. FLOW FROM LANDFILL EXAMPLE

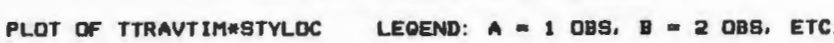

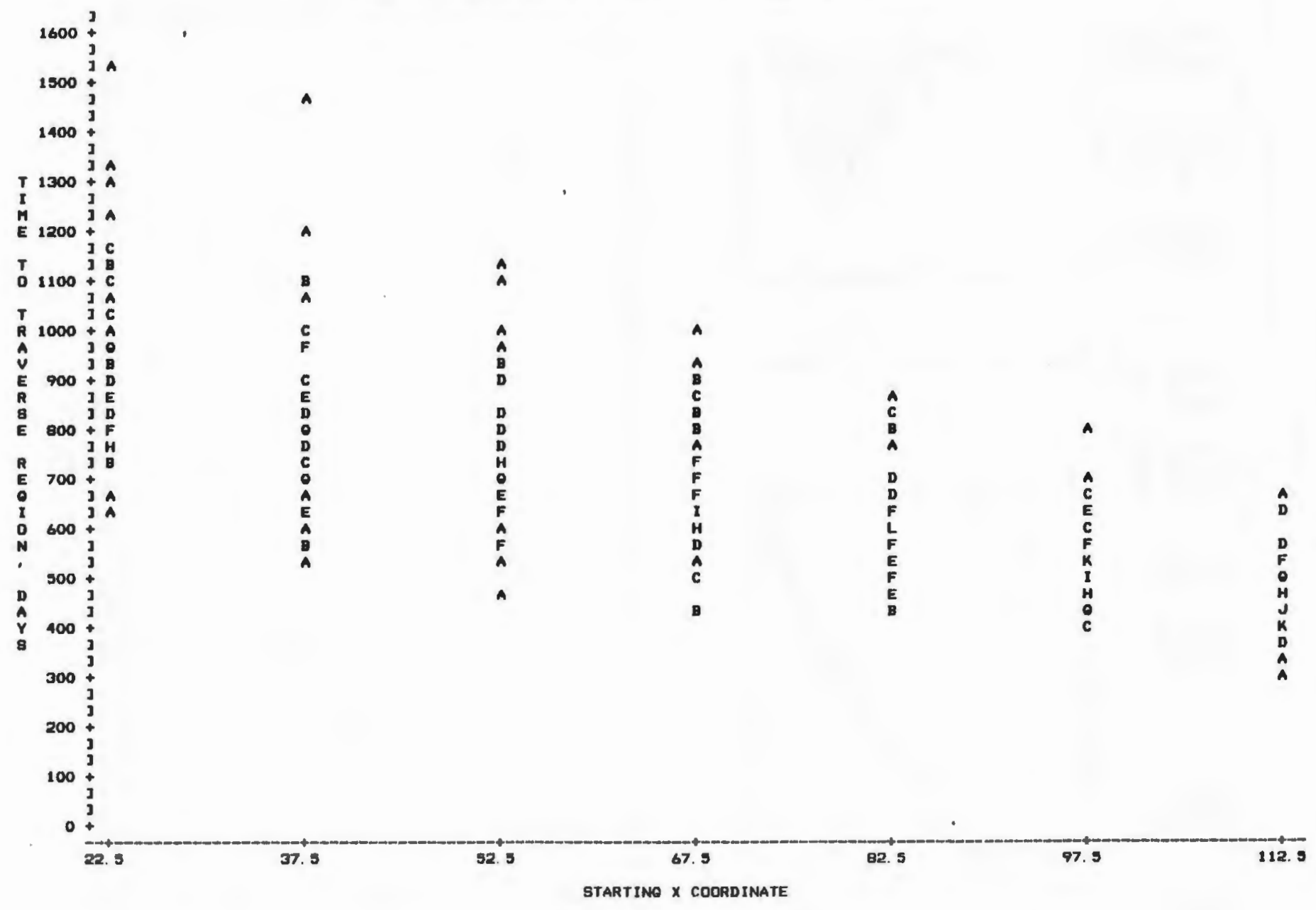

Figure 16 - Total Travel Time Versus Starting X Coordinate 
FILE 45, FLON FROM LAMDFTL EXAPLE

UNIVARIATE

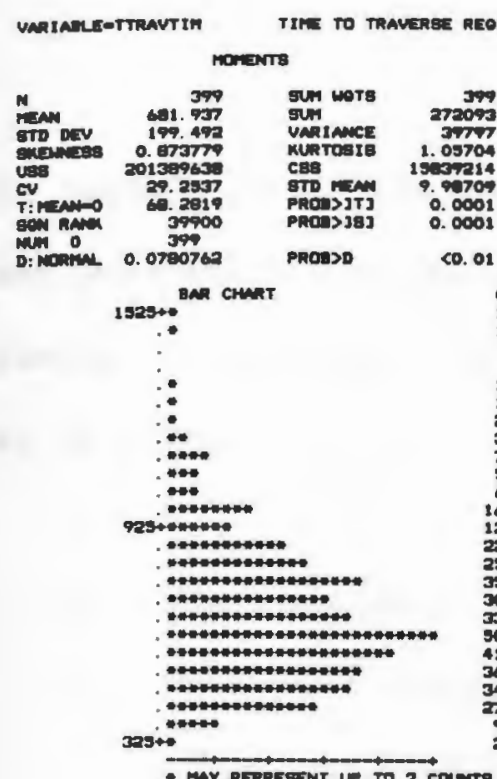

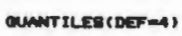

\begin{tabular}{|c|c|c|c|c|c|}
\hline \multicolumn{4}{|c|}{ OUNTI ILES (neF=4) } & \multicolumn{2}{|c|}{ Extereses } \\
\hline $\begin{array}{c}100 x \text { max } \\
75 x \text { os } \\
\text { sox } \\
25 x \text { ai } \\
\text { ox } \mathrm{mIn}\end{array}$ & $\begin{array}{l}1539.97 \\
600.091 \\
646.541 \\
534.119 \\
301.119\end{array}$ & $\begin{array}{l}99 x \\
95 x \\
90 x \\
10 x \\
9 x \\
12\end{array}$ & $\begin{array}{l}1284.77 \\
1081.96 \\
926.900 \\
447.914 \\
413.49 \\
337.946\end{array}$ & 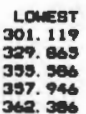 & $\begin{array}{l}\text { HIOAEST } \\
1242.02 \\
1284.78 \\
1318.91 \\
1469.89 \\
1539.97\end{array}$ \\
\hline
\end{tabular}

- mar aepresent u to 2 counts

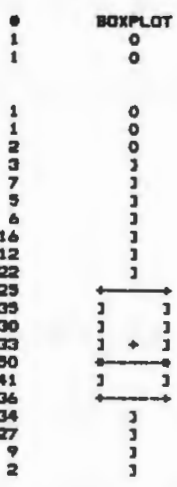

01.119

1 ง39. 97

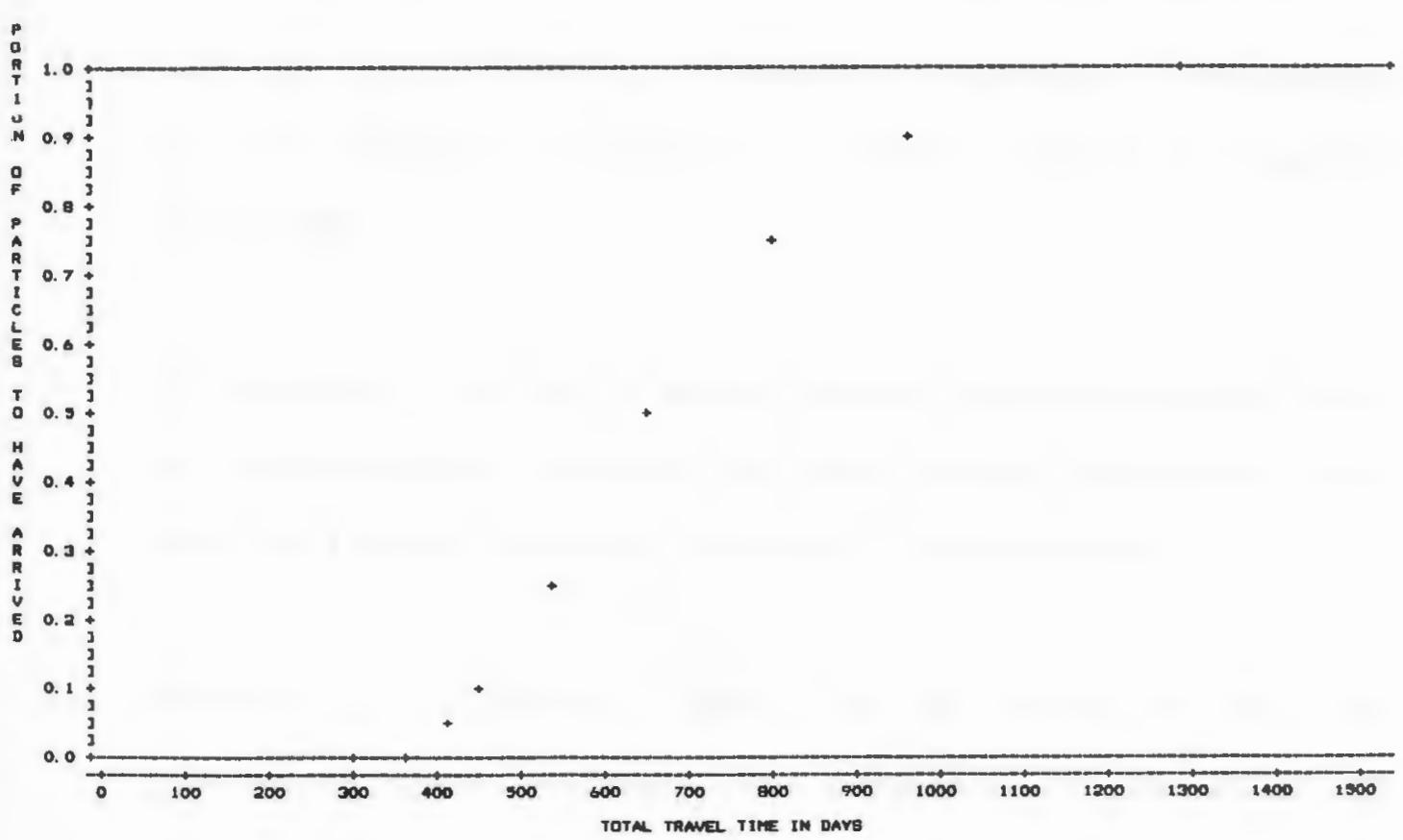

Figure 17 - Statistical Summary on Travel Time 
simulation, some confidence may be developed on the range of values of arrival time at the stream.

\section{CONCLUSIONS}

The usefulness of the methodology outlined in the companion paper has been demonstrated by two field problems. The following conclusions concerning the results of these examples in steady state groundwater flow may be drawn:

1. The geometric mean is the best predictor of the effective hydraulic conductivity in terms of flow rate and travel time after applying a shape factor.

2. Very few simulations are required to develop a consistent value of the mean flow rate and travel times in the regions considered.

3. The presence of a high hydraulic conductivity stratum at depth may significantly increase the flow through the region even when the flow is generally parallel to the stratum.

4. Estimates of confidence limits may be developed from the results of these analyses when normality is confirmed and assuming that the soil properties adequately characterize the in situ soil. 
5. Even when the distribution of travel times and flow rates cannot be accepted as conforming to a normal distribution, confidence may be developed in the predictions of these results by reviewing the histograms and cumulative mean results.

6. More work is needed to develop autoregressive parameters for sediments. It is not likely that these parameters will vary over a large range but more actual field studies are required to develop these parameters. 
APPENDIX-REFERENCES

1. Aldinger, Paul B. "Groundwater Flow Simulation By a Stochastic Representation of Soil", dissertation presented to University of Rhode Island, Kingston, Rhode Island, in 1983 in partial fulfillment of the requirements for the degree of Doctor of Philosophy.

2. Aldinger, Paul B. "Groundwater Flow in a Stochastic Medium: Model Development" Journal of Geotechnical Division, ASCE, to be submitted.

3. Casagrande, Arthur, "Research on the Atterberg Limits of Soils," Public Road, Vol. 13, Oct. 1932

4. Cedergren, H.R., Seepage, Drainage and Flow Nets. New York: John Wiley and Sons, Inc., 1967

5. Corps of Engineers, Big River Reservoir Project, Rhode Island, Vol. III, Appendix F - Geotechnical Analysis, July, 1980

6. Harr, M.E. Groundwater and Seepage, New York: McGraw-Hill Book Co., Inc. 1962

7. KAME, Preliminary Geotechnical Investigation, Phase 1, Vol. 1-2, Nov. 1977. 
8. Naval Facilities Engineering Command, Department of the Navy, Soil Mechanics, Foundations and Earth Structures: Design Manual 7, 1982.

9. Peck, R.B., Hanson, W.E. and T.H. Thornburn, Foundation Engineer ing, New York: John Wiley and Sons, 1953.

10. Smith, J. Hiram, Surficial Geology of the Crompton Quadrangle, Rhode Island, U.S. Geological Survey, 1956

11. Smith, I., "A stochastic analysis of steady-state groundwater flow in a bounded domain", dissertation presented to the University of British Columbia in Vancouver, Canada in 1978, in partial fulfillment of the requirements for the degree of Doctor of Philosophy. 
APPENDIX A

INTRODUCTION AND REVIEW OF THE PROBLEM

\section{Introduction}

Groundwater flow problems have always been particularly challenging due to their complexity and the uncertainties involved. Increasingly, predictions of flow quantity, heads, direction and/or travel time are necessary to answer questions on risk and safety. This need for reliable predictions has brought investigators from various disciplines within the sciences into the arena. The result is that considerable progress has been achieved in advancing the state of the art in recent years.

The complexity of these problems is derived from many factors. One factor is the difficulty in scaling up and averaging quantities at the microscopic or particle level to the macroscopic or field scale of the flow region. Another factor is the incomplete knowledge of flow regime caused by the complex conditions during deposition and/or subsequent weathering. Still other factors are the sheer number of variables and the limited resources available for analysis.

Over the years, numerous techniques have been developed to treat these groundwater flow problems. Analytical formulations which lead to exact solutions for the flow conditions, have been developed for many flow situations, (Harr 1962). Analytic techniques suffer from their lack of flexibility and the need to make restrictive simplifying assumptions for 
boundary conditions and media characteristics thereby inhibiting their use with most problems.

Perhaps the most widely used technique today is that of flow nets, see Casagrande (1937), Cedergren (1977). Its greatest advantage is its simplicity and ease in use. This technique is also limited in it's ability to vary soil hydraulic conductivity and groundwater free surface.

Scale models in sand tanks have also been utilized to study the flow of groundwater e.g. Bear (1961), Prickett (1975) (Grace 1981). Boundary conditions may be rather flexible but hydraulic conductivity variations cannot be easily simulated, capillarity action and grain size cannot be properly scaled and time is distorted. Other physical models such as the viscous fluid model have been utilized (Rushton and Redshaw, 1979) but they also require considerable expertise and specialized equipment.

A common characteristic of all these techniques is their lack of flexibility in boundary condition treatment and/or soil property characteristics. When these techniques are utilized, the consequences of the required averaging and other approximations are often unknown to the investigator.

With the recent interest in this area from the multitude of scientific disciplines and with the advancements in computer technology in the last decade, major changes in the way these flow problems are treated have occurred. Investigators have developed numerical solutions which allow 
much more latitude on boundary conditions and input parameters and therefore allow more realistic characterizations of flow regimes to be economically considered.

Numerical solutions are approximate solutions. However, when properly executed they result in solutions within the desired level of accuracy. The most common types of numerical solutions are the finite element method (FEM) and the finite difference method (FDM). Either of these methods may be used to solve most of the situations encountered, however, one method may have some advantage over the other for particular problems. A review of these methods may be found in Prickett (1975) and Wang and Anderson (1982).

These methods have provided the means to introduce not only more complex boundary conditions and input parameters but have allowed introduction of new considerations. Of these, stochastic generation of a correlated hydraulic conductivity distribution to represent the flow regions, stochastic simulation of microscopic dispersion and the utilization of probability techniques to quantify input and output are major considerations of this work.

\section{The State of the Art}

The methods outlined herein are intended to provide a tool for solving real problems. It is appropriate to consider the present state of the art for the types of problems for which this method will be appropriate. 
Universities and other research institutions have led the way in the development of numerical methods. Here finite element and finite difference methods are routinely used to study groundwater flow. Nunerous researchers have investigated the significance and usefulness of introducing variability of soil parameters to the problem. Some of these have proven fruitful; others have not.

Workers in government and industry who are faced with real field problems have generally relied on less sophisticated models for most problems. Numerical models have generally been utilized for only the most critical field problem where large amounts of money and data are available.

The advancement of these techniques in research work coupled with the tremendous increase in computing power available provides the potential to expand the use of these new techniques in all the areas of the field. It is to this technology transfer that this work is directed.

\section{Numerical Methods}

Both finite element and finite difference methods require that the region be represented as a system of blocks or elements. Mathematical expressions are written for each block or element and combined with the boundary conditions to yield a system of simultaneous equations which can then be solved for the unknown values, normally the hydraulic heads.

The finite difference method was chosen for this work for several 
reasons. Working computer codes have been available for some time using the finite difference method. They are fundamentally quite simple and adequate for most real problems. More practitioners are familiar with the finite difference method than finite element method and the numerical method was of secondary importance to the characterization of the flow region, the dispersion studies, and output analysis.

The basis of these numerical models are Darcy's Law and the continuity equation. Darcy (1856) conducted a series of experiments to observe what factors governed the flow of water through a sand column. Darcy reported a direct relationship between the flow rate, $Q$, and the crosssectional area, $A$, and head drop, $h_{2}-h_{1}$. An inverse relationship was found between the flow rate and the length over which the head drop occurred, $1_{2}-1_{1}$. The resulting equation is given below with the negative sign inserted to indicate that the flow occurs in the direction that head decreases.

$$
Q=-K A \frac{\left(h_{2}-h_{1}\right)}{1_{2}-1_{1}}
$$

In this expression, $K$, is the proportionality constant called hydraulic conductivity.

The term, hydraulic conductivity, is utilized here and elsewhere (i.e., Freeze and Cherry, 1979) for this proportionality term rather than the permeability term most often used in the civil engineering field. Hydraulic conductivity is a function not only of the flow media but also of the pore fluid. When the effects of the pore fluid are extracted 
from this term, a second proportionality constant is left which is a function of the flow media only. This second term is also at times referred to as the permeability, the specific permeability or the intrinsic permeability. The adoption of the term hydraulic conductivity for the proportionality constant in Darcy's equation is made to avoid confusion with work done in other disciplines as well as a conviction that eventually this convention will be adopted by all workers in the field.

Laplace's Equation has been derived from the continuity equation for groundwater flow and Darcy's Law by others (i.e., Wang and Anderson, 1982) and is presented below:

$$
\frac{\partial^{2} h}{\partial x^{2}}+\frac{\partial^{2} h}{\partial y^{2}}+\frac{\partial^{2} h}{\partial y^{2}}=0
$$

This is the governing equation for groundwater flow in an isotropic homogeneous aquifer in three dimensions under steady state conditions. This equation becomes the basis of the solution by the finite difference method.

To understand the basic concepts, a simplified discussion in two dimensions follows. Figure A.I presents the overall flow problem to be represented for a two dimensional region as well as a close-up of one section of the finite difference mesh. Here the blocks are node centered and the head value is constant in each block. The size of the blocks is $\Delta x$ by $\Delta y$. The head at nodal point $i, j$ is signified as $h_{i j}$. The forward finite difference approximation of the first 
IMPERVIOUS BOUNDARY

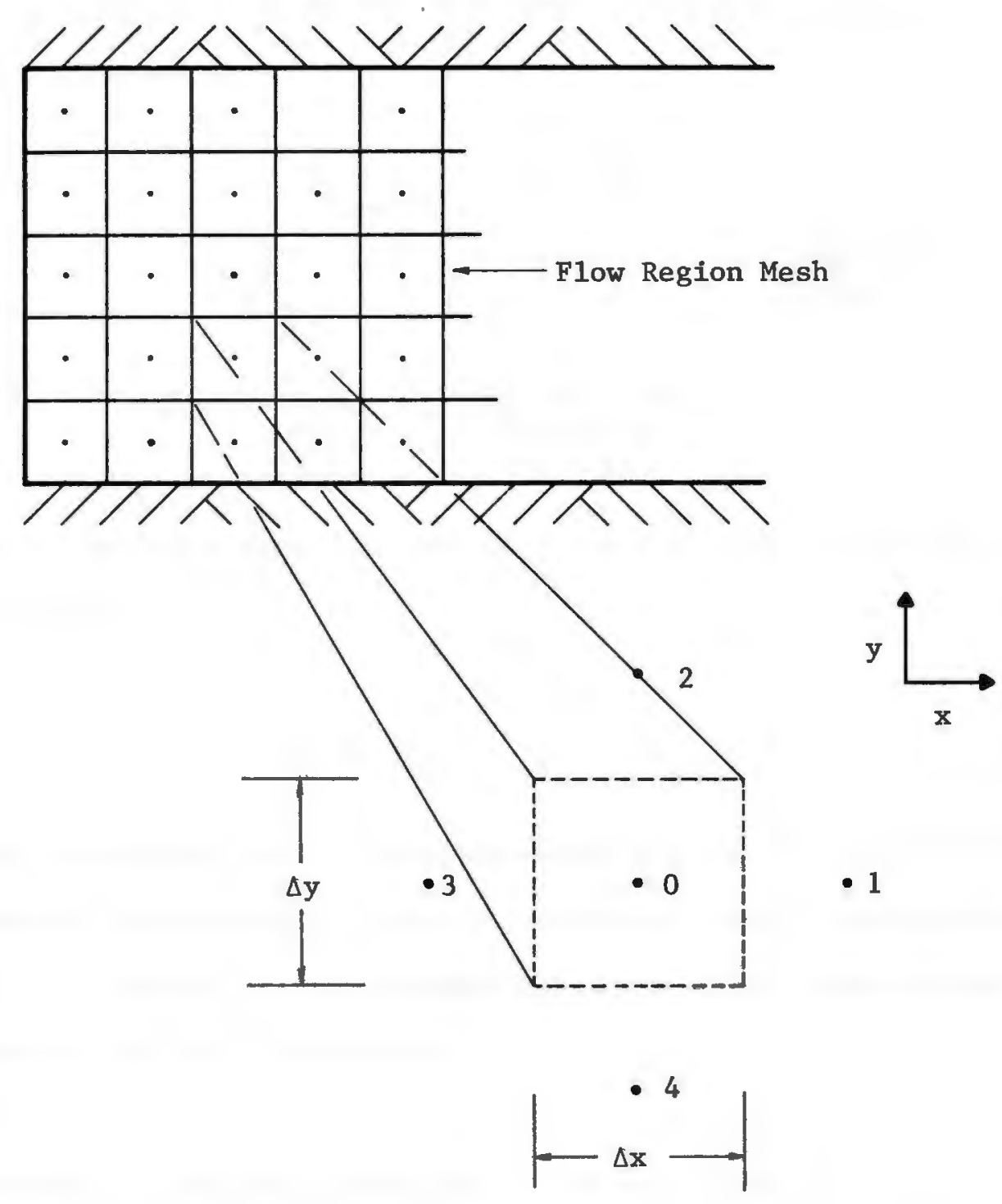

Figure A.1. - Flow Region Representation 
derivative, $\partial \mathrm{h} / \partial \mathrm{x}$ is $\Delta \mathrm{h} / \Delta \mathrm{x}$ which for nodes 0 to 3 is

$$
\frac{\Delta h}{\Delta x}=\frac{h_{0}-h_{3}}{\Delta x}
$$

The second derivative is approximated as the difference between adjacent first derivatives. Thus for the case considered in Figure A.1, the second derivative is:

$$
\begin{aligned}
& \frac{\partial^{2} h}{\partial x^{2}} \simeq \frac{\frac{h_{1}-h_{0}}{\Delta x}-\frac{h_{0}-h_{3}}{\Delta x}}{\Delta x}=\frac{h_{1}-h_{3}-2 h_{0}}{(\Delta x)^{2}} \\
& \text { and } \quad \frac{\partial^{2} h}{\partial y^{2}} \simeq \frac{h_{2}-h_{4}-2 h_{0}}{(\Delta y)^{2}}
\end{aligned}
$$

Utilizing Laplace's Equation, and if $\Delta x=\Delta y$, then we get the equation for this node:

$$
h_{1}+h_{2}+h_{3}+h_{4}-4 h_{0}=0
$$

Similar expressions may be written in succession for all the nodes in the region, substituting boundary conditions where appropriate. The result is a series of simultaneous equations which, when solved, yield the head values at all the nodes.

Many techniques have been utilized to perform this job. Reiter (1981) adopted the computer code developed by the USGS in their 1976 finite difference model as described in Trescott, et. al (1976). The Iteriative Alternating Direct Implicit (IADI) technique described by Reiter (1981) was used to determine the head values at the nodal points 
in the flow region for the problem's boundary conditions in this work.

IV. Boundary Conditions

The conditions at the edges of the flow region which must be entered to obtain a solution are the boundary conditions. These conditions define the limits of flow and make the solution of flow within the region unique.

Boundary conditions are of two general types: constant flow, often no flow, and constant hydraulic head. It is quite common to have combinations of both types in each field problem.

The initial boundary condition considered is one of shape or configuration of the no flow boundary. This is really a type of constant flow condition where some nodes have no flow. The simplest example of this is one dimensional flow such as flow in a narrow pipe. Flow conditions are relatively simple here and will get progressively more complicated as flow in two or three dimensions are considered. It is clear that real field problems are three dimensional problems, however, the study of three dimensional flow can require enormous amounts of data and effort to solve when compared to two dimensional problems. Fortunately, flow in two dimensions can often represent field conditions reasonably well and therefore most studies still rely on two dimensional models.

Another restriction is the need to limit the flow regime. In two dimensional flow the simplest region is a square or rectangular region as 
presented in Figure A.1. This configuration often adequately models the flow region. However, oftentimes an irregularly shaped region can more accurately describe the flow region and thus the boundary conditions must be modified.

This initial type of boundary condition generally limits flow at the boundary. This may be necessary to simulate no flow through impervious strata as across groundwater divides. At no flow vertical boundaries, the flow in the horizontal or $x$ direction is zero so that $\partial \mathrm{h} / \partial \mathrm{x}$ must also equal zero. Figure A.2 presents a typical problem in two dimensional groundwater flow with its corresponding boundary conditions.

The second type of boundary condition was that of hydraulic head regulation. This condition basically maintains a consistent head of known magnitude at the boundary, either constant or otherwise. Irrespective of conditions within the region, the head values are unchanged at these boundaries. The reason for setting these conditions in the problem may be to simulate a surface water body such as a lake or stream.

For real problems, the boundary conditions are usually determined with the use of observation wells and careful study of the region to locate significant water bodies and other indications of the groundwater gradient. No flow boundaries must also be identified and recognized either in very impervious strata or groundwater divides.

V. Characteristics of the Region 

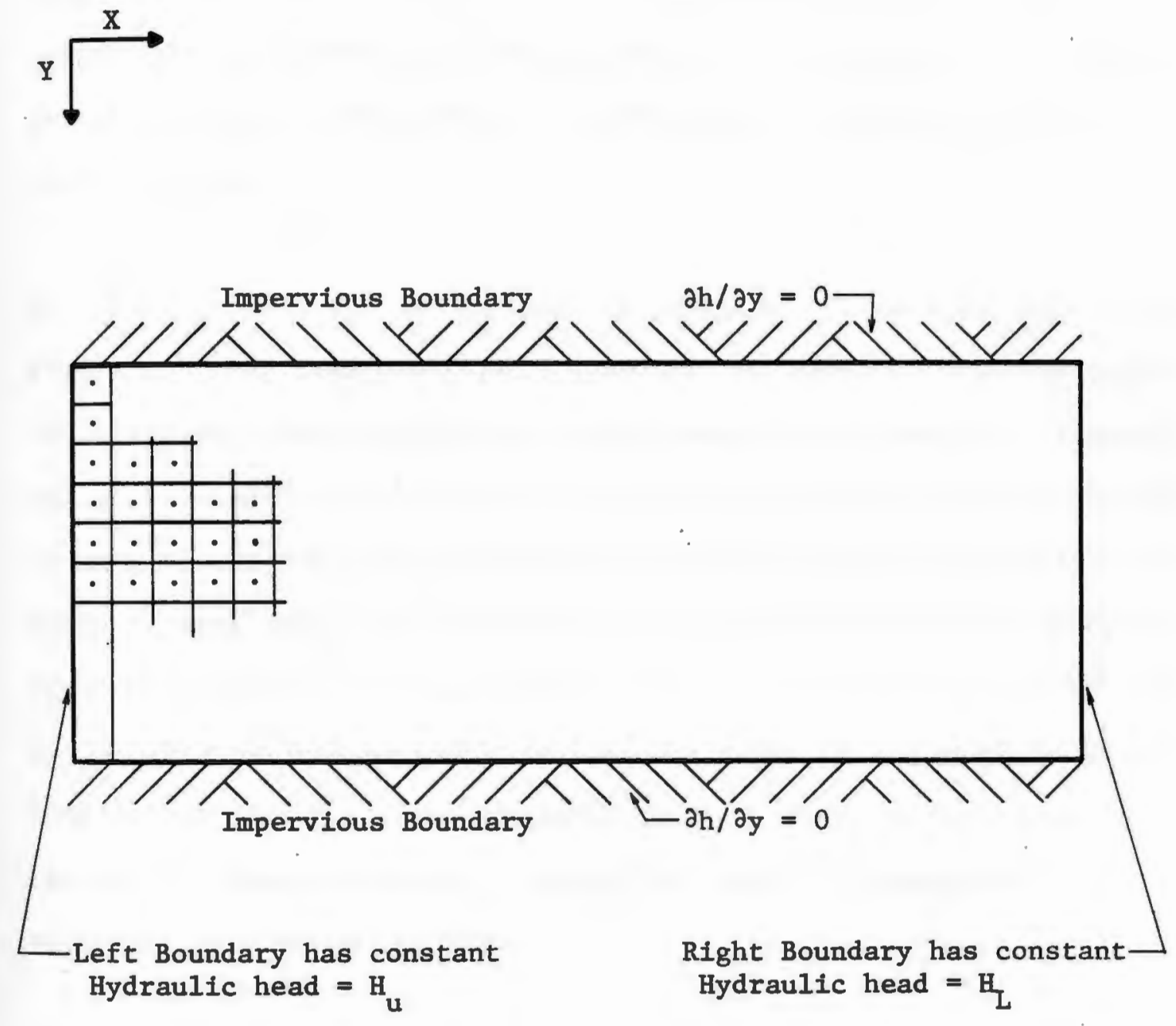

Figure A.2. - General Flow Region 
Perhaps the most significant input to the flow problem solution is that of the characterization of hydraulic conductivity. The range of values which this single parameter may realistically have is very broad. Hydraulic conductivities in natural deposits of soil have been found to vary by thirteen orders of magnitude. Freeze and Cherry (1979) indicate that the hydraulic conductivity of some gravels may be as high as 100 centimeters per second and that of some clays as low as $10^{-11}$ centimeters per second. The hydraulic conductivity of some rocks may even be lower than this.

It is not common for an engineering parameter to vary by this much. Figure A.3 from Cedergren (1967) compares the range of possible values for hydraulic conductivity with other common soil parameters: strength and unit weight. It has been found that the same soil type may vary by as much as six orders of magnitude and the same stratum by more than one order of magnitude. This information suggests that simply knowing the order of magnitude of the hydraulic conductivity will be useful and that it is unlikely that we can determine the value at any point to within much closer than an order of magnitude. We also can expect to see a relatively large variation, perhaps an order of magnitude or more, within a "homogeneous" deposit.

Geologists, geotechnical engineers, irrigation engineers, and petroleum engineers, among others, have attempted to develop methods to estimate the value of hydraulic conductivity insitu. Estimates have been attempted by testing "undisturbed" samples in the laboratory, by empirical relationships with grain size and density, and by field testing, includ- 


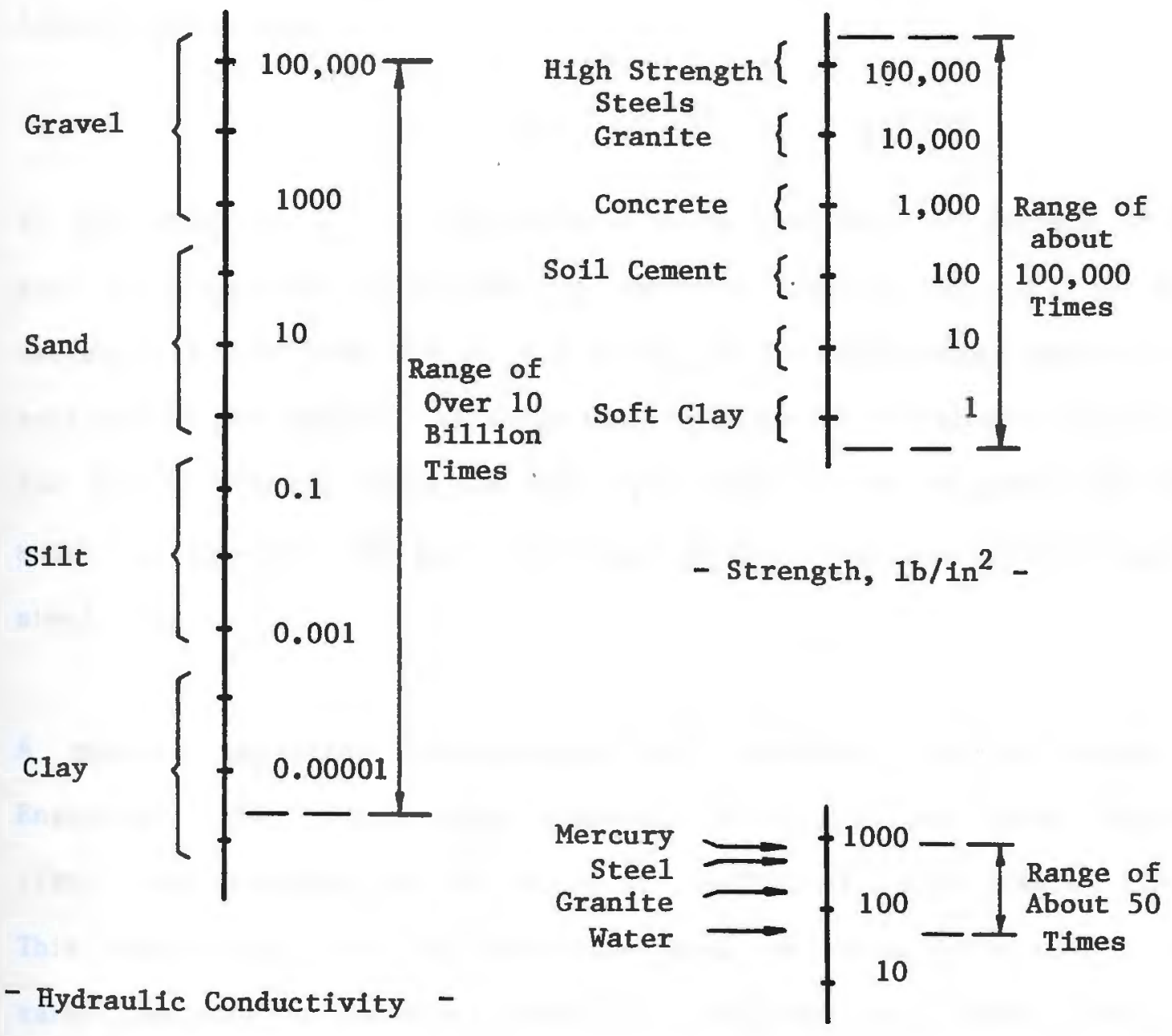

Ft/Day

- Unit WeIght, $1 \mathrm{~b} / \mathrm{Ft}^{3}$ -

Figure A.3 - Variability of Soil Engineering Properties, after Cedergren (1967) 
ing field pumping tests and outflow tests. The results of these many attempts suggest that no method is very accurate and there are advantages and disadvantages for each of the methods adopted.

Estimates utilizing grain size distribution have been tried by many investigators. Perhaps the most commonly used equation is the Hazen formula given below.

$$
K=c\left(d_{10}\right)^{2}
$$

In this equation $d_{10}$ is the particle grain size which 90 percent of the soil is larger and 10 percent is smaller. The constant, $C$, has been estimated to be from 1.0 to 1.5 if $d_{10}$ is in millimeters and $K$ is in centimeters per second. Although this equation was developed originally for fairly uniform sands, it has been found to be adequate for most sands and gravels. The major advantage of this equation is its relative simplicity.

A similar empirical relationship was developed by The Corps of Engineers. The relationship presented in Figure A.4 from Leonards (1962) was developed on the basis of results of field pumping tests. This relationship was also based on tests of sands and gravels. The range predicted by the Hazen formula was superimposed on this figure for comparison purposes.

Other more complicated formula have been developed which seek to incorporate other factors which have a demonstrated effect on the hydraulic conductivity values. Some of these factors are the sorting or uniform- 


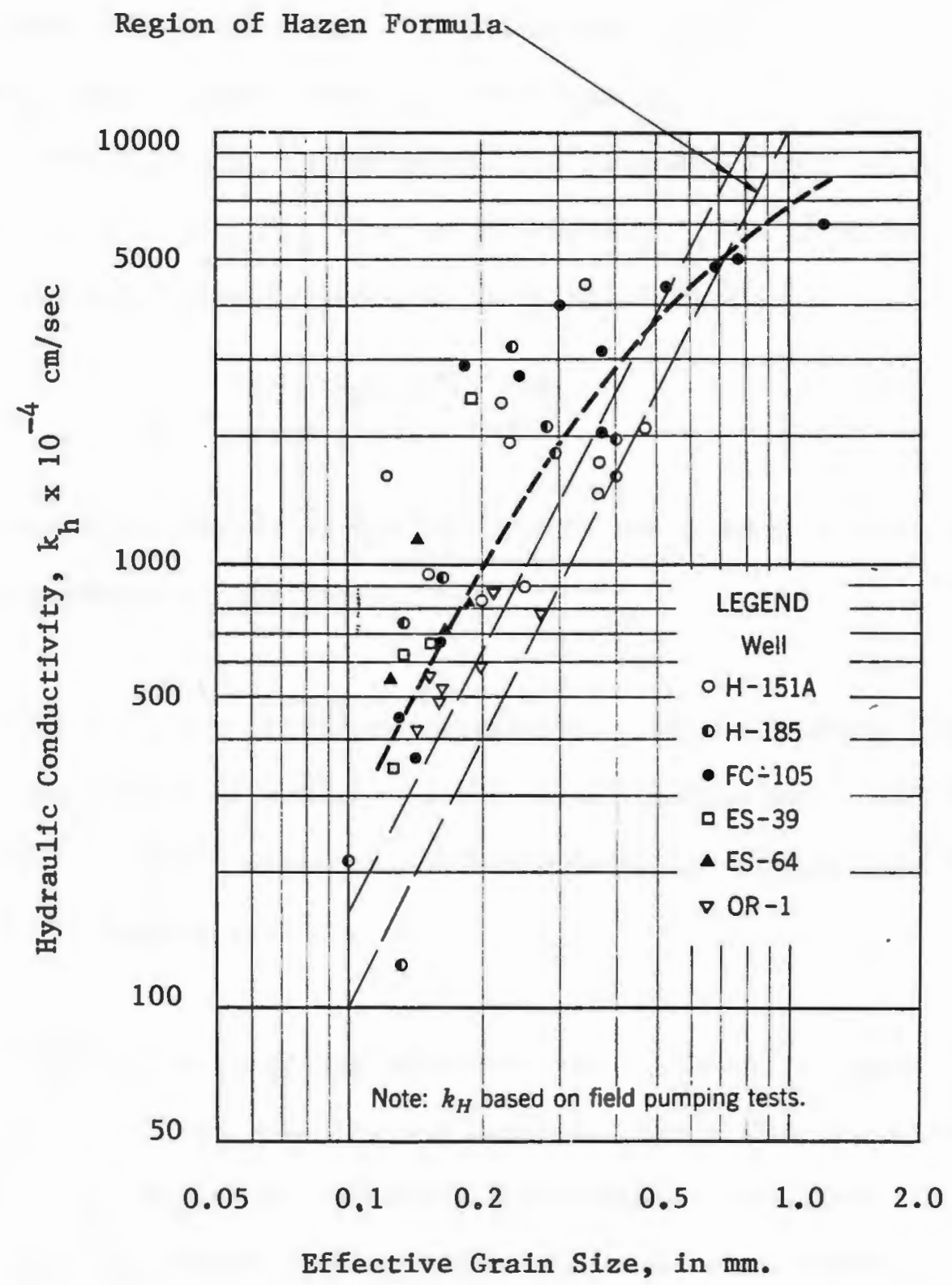

Figure A.4 - Empirical Relationship Between Grain Size and Hydraulic Conductivity, after Leonards (1962) 
ity of the grain sizes in the soil. A soil which is well sorted contains mostly one particle size and the hydraulic conductivity will tend to be higher. A soil which is not well sorted will be well graded, the smaller particles filling the voids between the larger particles and thus lower hydraulic conductivity. An example of these is one developed by Masch and Denny (1966) given in the form of a semi-log plot in Figure A.5. The grain size curves plotted in Krumbein $\emptyset$ units where $\emptyset=$ $\log _{2} d$ and $d$ is the particle size in millimeters. The sorting aspect takes the form of a factor referred to as $\sigma_{I}$ which equals

$$
\sigma_{I}=\frac{d_{16}-d_{84}}{4}+\frac{d_{5}-d_{84}}{6.6}
$$

These relationships were developed for sands and gravels tested under controlled conditions in the laboratory.

Prugh developed a series of curves dependent on the uniformity coefficient for three levels of density, i.e., loose, medium dense and dense (Powers, 1981). These empirical relationships were developed with results of field pumping tests.

Another soil parameter affecting hydraulic conductivity is porosity or void ratio since the two are directly related. The higher the porosity or void ratio, the higher the hydraulic conductivity, all other factors the same. The best known equation which includes this factor is the Kozeny-Carmen equation (Bear 1972) given below:

$$
K=\frac{P_{g}}{u} \quad \frac{n^{3}}{(1-n)^{2}} \quad \frac{d_{m}^{2}}{180}
$$




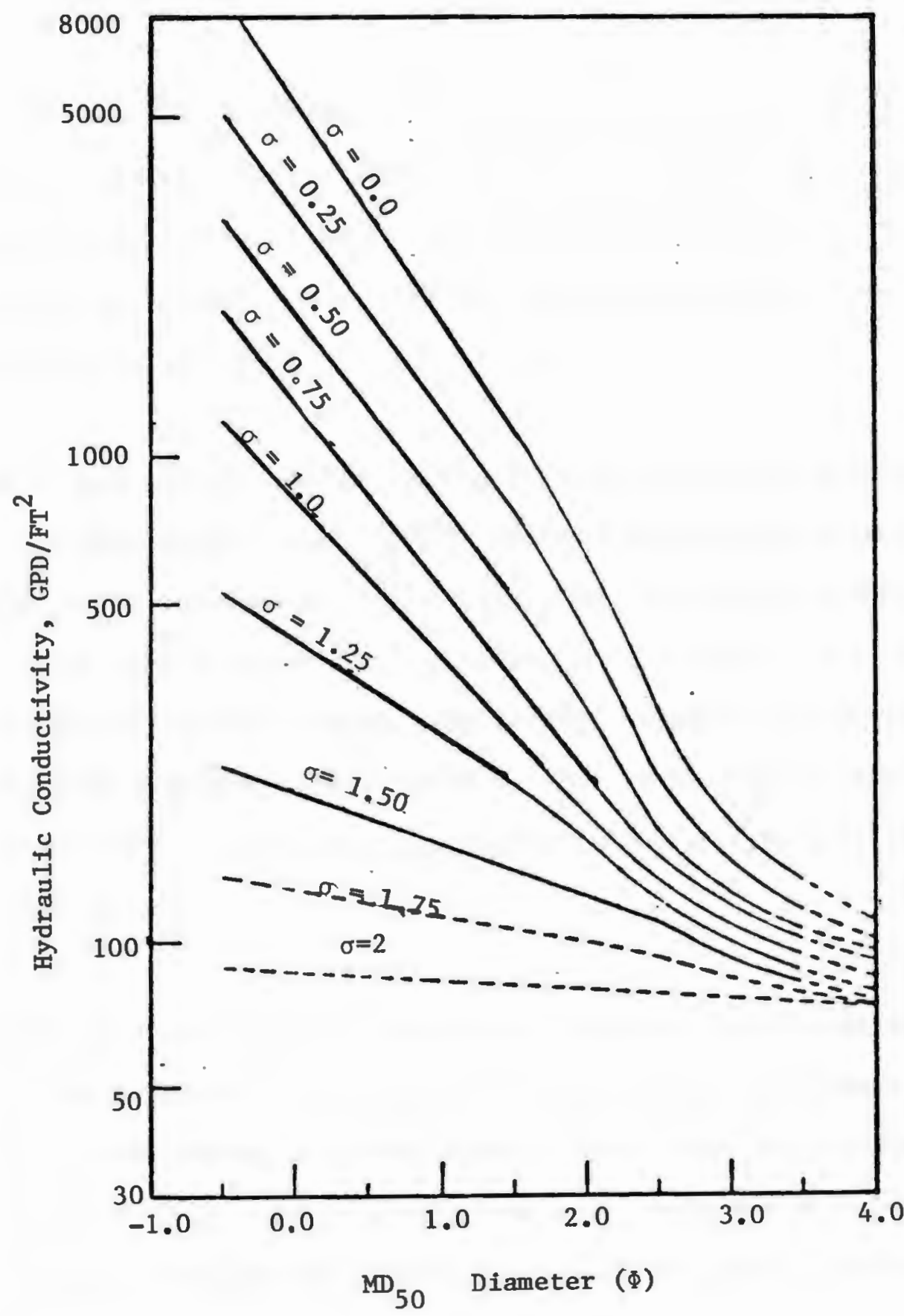

Figure A.5 - Other Empirical Relationship Between Grain Size and Hydraulic Conductivity, after Masch and Denny (1966) 
In this formula, $p$ is the density and $u$ is the viscosity of the fluid, $g$ is the acceleration of gravity and $d_{m}$ is the representative grain size.

Other less significant factors such as particle shape and packing have also been suggested, Todd (1980), for correlation with hydraulic conductivity. The effects of these other factors are relatively insignificant when compared with those already discussed, and therefore are not generally accounted for.

Laboratory testing of samples of the soil may yield quite reasonable values for some soils. Lambe (1951) presents the procedures most often utilized in an engineering soils laboratory to conduct these tests. Voids along the edge of the permeameter may affect the results, particularly of cohesive soils. Undisturbed samples of granular soils are virtually impossible to collect so that reconstituted samples are generally tested. These modifications of the samples may also severely modify the results.

Field testing of soil inplace consists of generally two techniques. The best is often referred to as slug or outflow tests. Such tests consist of simply introducing or withdrawing water into a piezometer or observation well and observing the quantity of flow for constant head maintenance or the speed of water surface drop or rise. NAVFAC (1982) presents a good summary on how these tests may be performed and analyzed. The major problems with these tests are that some water may be pushed up voids along the casing, or a collection of silt on the bottom of the casing may distort the results. The test generally 
represents only a small volume of soil and the hydraulic resistance of the screen, if any, is not accounted for.

The other type of field test is the pumping test. This test generally consists of pumping from a screened well at a known flow rate and observing drawdowns in observation wells around the well. Stallman (1971) presents a good narrative on designing and analyzing a pumping test. This test is perhaps the most realistic of all the tests conducted but suffers from the fact the hydraulic conductivity value determined is some mean of all those insitu so that a sense of variability is not clear. It also is handicapped by the fact it is very expensive and can only be utilized when its high cost can be justified.

In light of the fact that the value of hydraulic conductivity is highly variable, many investigators have chosen to consider this parameter as a random variable. A random variable is a variable whose precise magnitude cannot be determined with certainty, a priori. This is certainly the case for hydraulic conductivity. Probability aspects will be discussed later in this appendix.

Another input parameter has already been mentioned. This is porosity which is defined as the volume of voids divided by the total soil volume, expressed in percent. Porosity is related to hydraulic conductivity but its maximum range of values is only from zero to $100 \%$. Additionally, typical values for soil are only within the range of 15 to 60 percent. Porosity is utilized to calculate the velocity of groundwater flow since the Darcy velocity is not the actual water particle 
velocity but really a homogeneized term.

The other remaining input parameters are the microscopic or particle scale longitudinal and transverse dispersivities. The dispersivity is a property of the soil which relates to the spreading of the water particles about a mean direction of movement. This term attempts to incorporate the spreading due to the tortuous path followed as the water particle traverses the flow region. Dispersivity has been found to be an approximate function of the grain size of the porous media, Kelly (1982). Kelly pointed out the difficulty in scaling up sand column dispersivities from the laboratory to a field problem. A discussion on dispersion will follow in a later section.

VI. Stochastic Soil Modeling

Recent work in the study of groundwater flow indicates that engineers and geologists now recognize that it is oftentimes dangerous to characterize soil properties simply with some average value or some other presumably "conservative" estimate. Even the addition of multi-strata flow regions and sensitivity analyses on input parameters are not always satisfactory. Recently investigators have turned to the use of probability and statistics to facilitate their understanding of these complex problems.

Simple statistics have, of course, long been utilized to characterize soil properties, i.e., the average or mean value. However, it has been only recently that the randomness of the data, to some extent quantified 
by its standard deviation, has been incorporated into the problem analysis e.g. Harr (1977). Other more powerful concepts are available and are now also being used.

There are many good introductory texts dealing with probability and statistics for engineers and scientists, e.g., Benjamin \& Cornell (1970), Miller and Freund (1977) and Walpole \& Myers (1978). It is not the purpose of this appendix to introduce these concepts since they are readily available elsewhere, however, some discussion will follow on their usage in the study of groundwater flow.

It is very unlikely that a soil property such as hydraulic conductivity or porosity will be uniform in situ. Variations are inevitable. However, when analyzing groundwater flow, the investigator must, by necessity, recognize similarities and divide the region into like strata to facilitate analysis.

Soil media involves numerous characteristics which may be considered as random rather than deterministic variables. This implies that the exact value of these characteristics or properties at any point is not known and cannot be predicted with certainty, a priori. There is uncertainty in any estimate prior to experimentation.

The principal difference between stochastic and deterministic soil modeling is that the former recognizes variability and attempts to quantify its effects. Since the probability distribution of a random variable describes the limits of its variability in probabilistic terms, 
it may be used to generate a significant number of equally probable representations of the soil medium. The ensemble of solutions to the flow problem provides input on the variability of these results. This repetitive simulation of the flow media and analysis of the ensemble of results is commonly referred to as a Monte Carlo simulation.

Most soil characteristics may be considered continuous random variables, i.e., may have non-integer values. The probability distribution relationship is therefore the probability density function (PDF) which simply relates the respective value of the variable with the corresponding probability.

Figure A.6 presents a typical PDF and the equation for this function which has been found to adequately characterize the characteristics of some soil properties. e.g., Lumb (1974) and Harr (1977). This PDF is for the Gaussian, or normal distribution, which is also the most commonly studied distribution. This distribution is completely defined by two parameters, the mean and the standard deviation.

A unique aspect of the normal distribution proven by probability theory is the Central Limit Theorem. The hypothesis of this theorem is that the normal distribution will approximate a random variable which is itself the sum of many other random variables. Benjamin and Cornell (1971) state the theorem as follows:

"Under very general conditions, as the number of variables in the sum becomes large, the distribution of the sum of random variables will approach the normal distribution." 

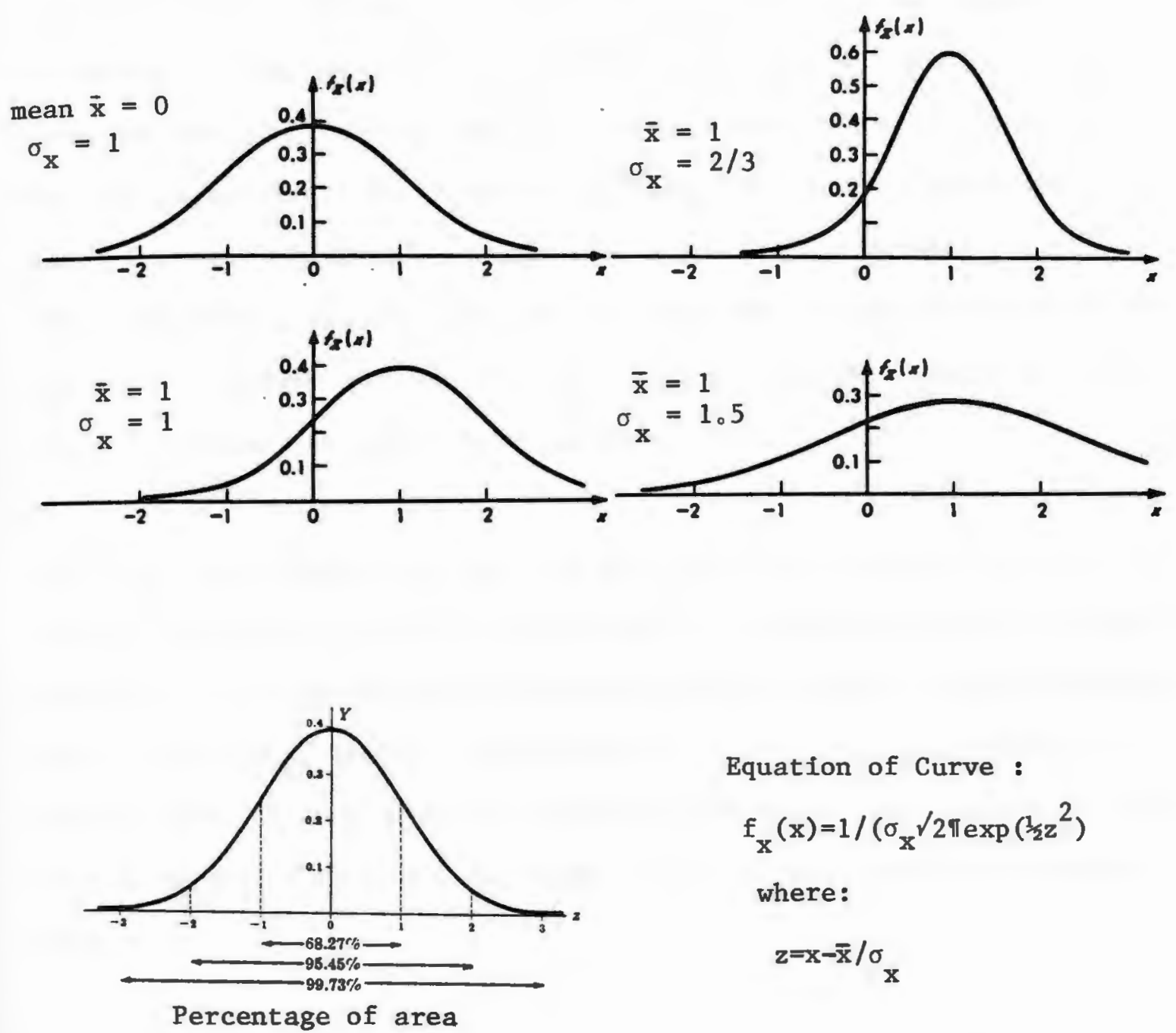

Equation of Curve :

$f_{x}(x)=1 /\left(\sigma_{x} \sqrt{ } 2 \pi \exp \left(\frac{1}{z} z^{2}\right)\right.$

where:

$\mathrm{z}=\mathrm{x}-\overline{\mathrm{x}} / \sigma_{\mathrm{x}}$

Figure A.6 - Probability Density Function for the Normal Distribution 
A second PDF which is the result of multiplying many other random variables is the $\log$ normal distribution. Figure A.7 presents several forms of the PDF for the log normal distribution with different values for the parameters. The equation for this PDF is also presented. Two aspects of the $\log$ normal distribution which have encouraged its use for some engineering aspects are that it fits data which is skewed and it precludes negative values for the random variable. Both of these characteristics are common in engineering data.

It should be pointed out that the log normal distribution may be considered a special case of the normal distribution. If a random variable, $K$, is $l o g$ normally distributed with a mean of $\bar{K}$ and a standard deviation of $\sigma_{k}$, then a new variable, $Y=\ln K$, may be defined. This new variable, $Y$, will have the normal distribution. In Appendix $B$, this fact is utilized to handle a random variable with a log normal distribution.

A useful technique of statistics which may be used to infer the probability distribution of a random variable is the transformation of variable technique. Freund and Walpole (1980) present a proof of this technique and some examples of its use. This technique uses the known relationship between two variables and the nature of the probability distribution of one of them to determine the probability distribution of the other.

Soil properties which are treated as random variables need not be categorized into a specific probability distribution with its known PDF. 


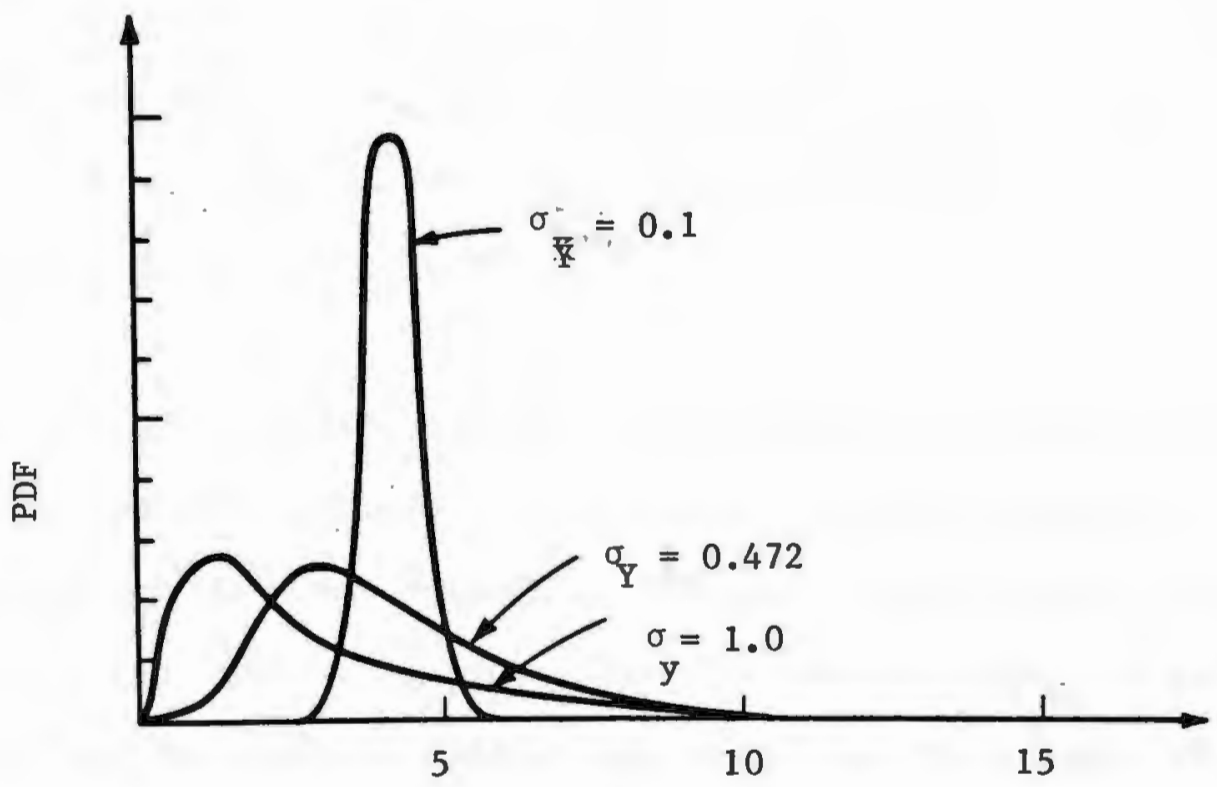

Bar Strength in $\mathrm{ksi}$

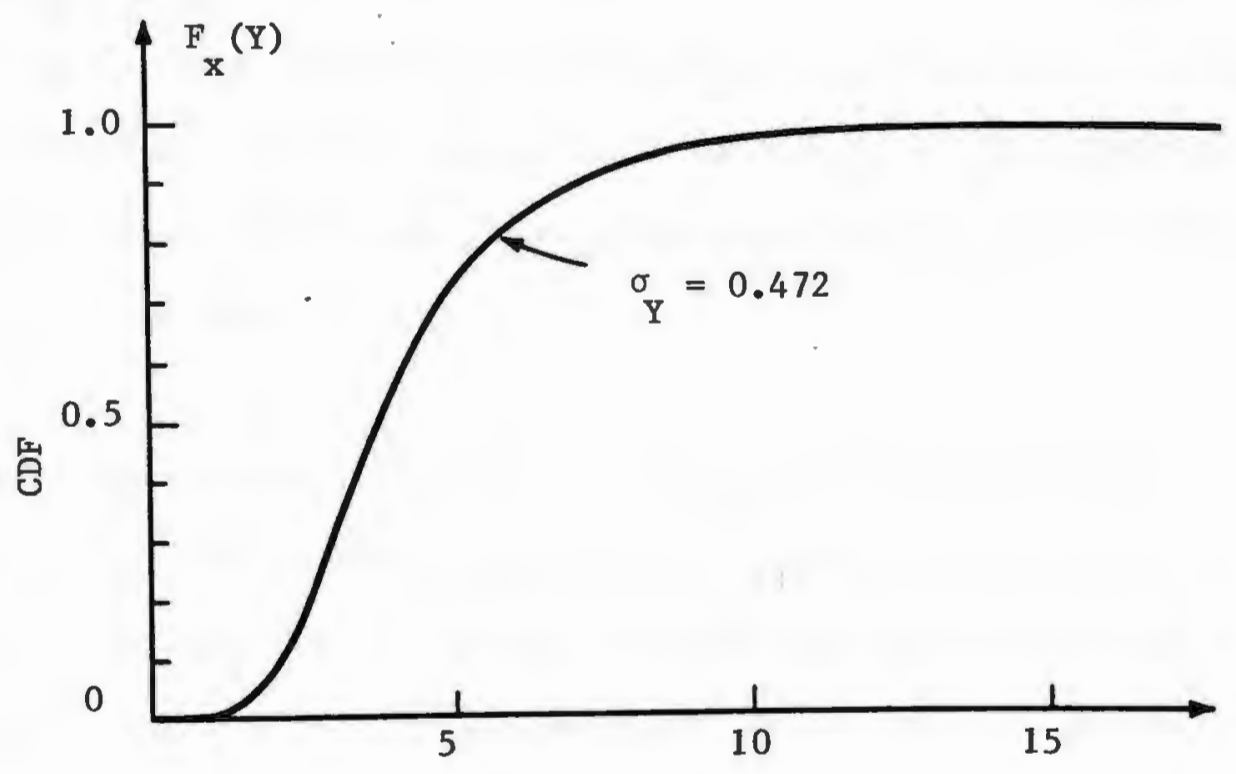

Bar Strength in ksi

Figure A.7 - Probability Density Function for the Log Normal Distribution, after Benjamin and Cornell (1970) 
However, where this is possible, considerably less data is required to develop confidence in the prediction of the soil property at any given point. Of particular concern with the strategy of matching data with a known PDF are problems in the tails of the distribution, i.e., the extreme values of the distribution.

There are several accepted methods to verify that an assumed PDF properly models the data collected. The probability and statistics texts cited earlier treat these methods in detail. These methods may be divided into two main types, graphical and computational. Graphical techniques may be used to roughly determine if the assumed PDF is adequate. Comparison of histograms generated from collected data and from the assumed PDF such as those in Figure A.8 may be used as a qualitative measure of acceptance. Figure A.8 after Benjamin and Cornell (1971) also presents a plot of the same data on normal probability paper. Data from a normal distribution will plot as a straight line on this paper so that a measure of conformity to the distribution is possible. Large deviations from a straight line would cast doubt on the assumption of normality.

Two quantitative techniques which are utilized to test the appropriateness of a PDF to model observed data are the chi-squared and Kolmogarov - Smirnov tests. These techniques quantify the deviation of the observed data from the assumed PDF or cumulative distribution function (CDF) within a specified significance level. The most popular of these is the chi-squared test although it suffers from the fact that for continuous variables it requires dividing the range of values into 


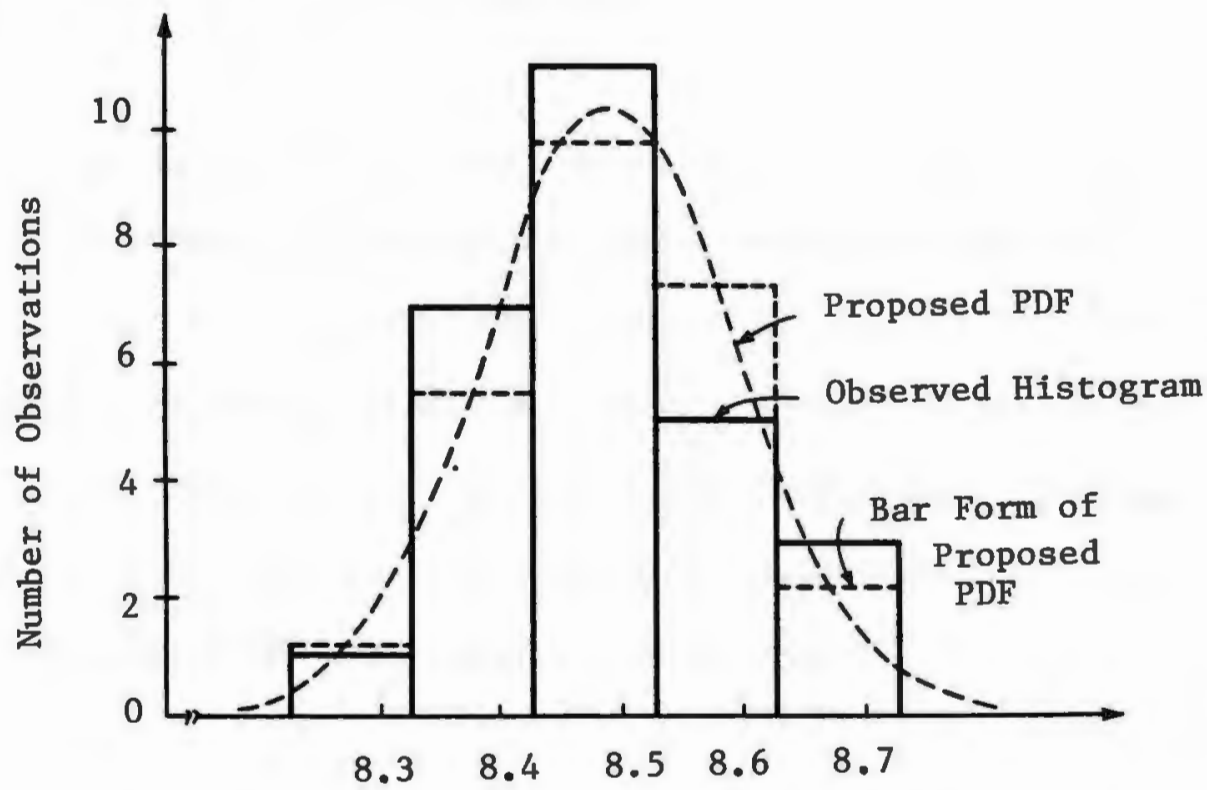

Bar Yield in ksi

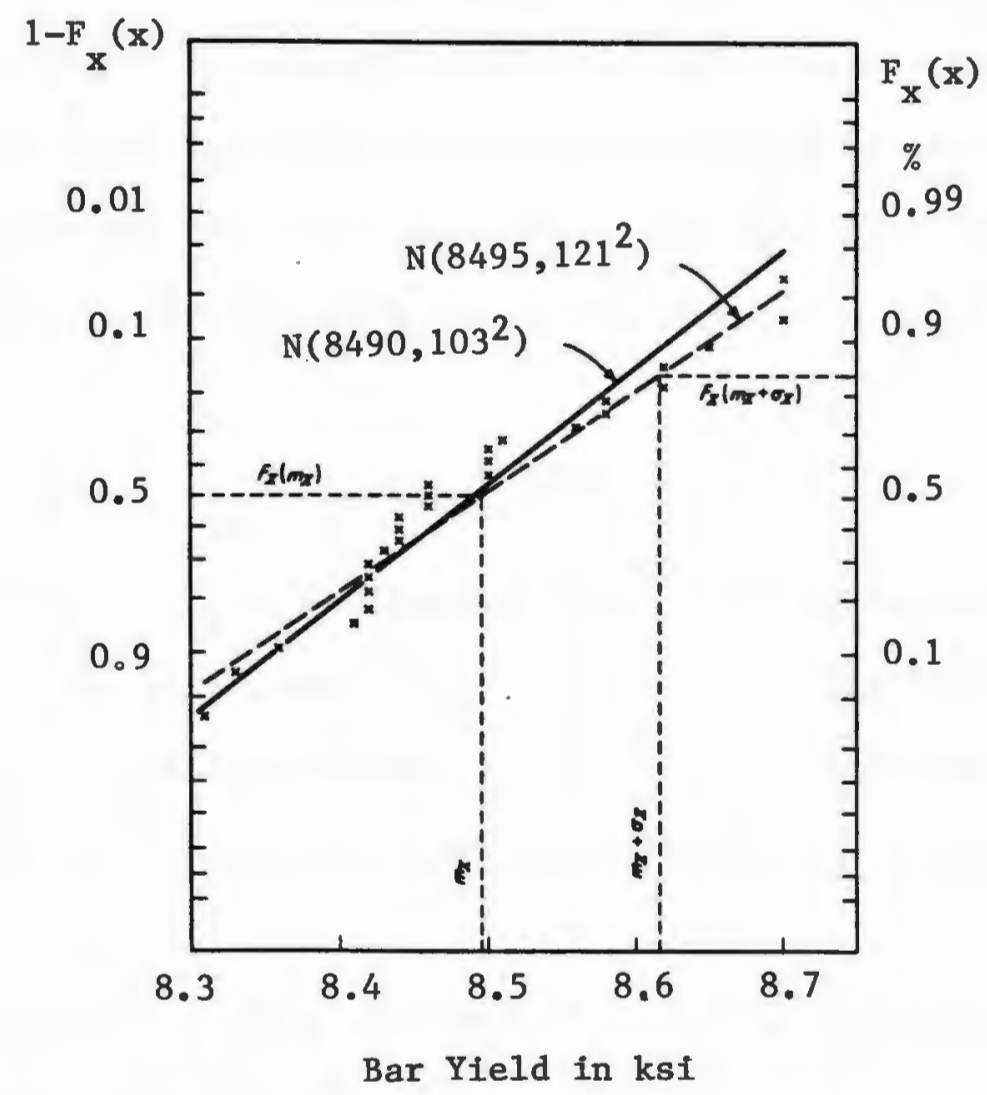

Figure A.8 - Example of Use of Histogram, Probability Density Function and Normal Probability Paper, after Benjamin and Cornell (1970) 
discrete intervals prior to testing significance.

In spite of these problems fitting data into these known probability distributions, numerous soil properties have been categorized by various investigators. Table A.1 compiles the results of several of these attempts. Although this review should not be considered exhaustive, it does indicate a consensus for some of these soil properties. Of particular interest to this work is that hydraulic conductivity has been found to have a log-normal PDF and porosity a normal PDF.

With the extreme variability possible for input parameters and output, a measure of confidence in the predictions is desirable. Research work in this area has resulted in several methods for estimating confidence limits on the mean values and estimates of the error in these estimates. Miller and Freund (1977) show that for a normal distribution, the maximum error, $E$, in the prediction of the mean value of the population is:

$$
E \leq Z_{\alpha / 2} \quad s / \sqrt{\mathbb{N}}
$$

In this inequality $\mathrm{Z}_{\alpha / 2}$ is a tabulated value for the normal distribution, such that the area under this distribution to its right is equal

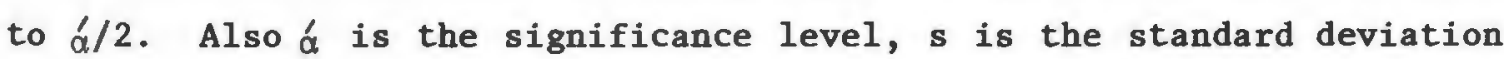
of the sample and $N$ is the number of observations in the sample.

Similarly, they also develop estimates of confidence limits for the population mean, $\overline{\mathrm{X}}$ as follows: 
Table A.1 - Probability Density Functions for Several Soil Properties

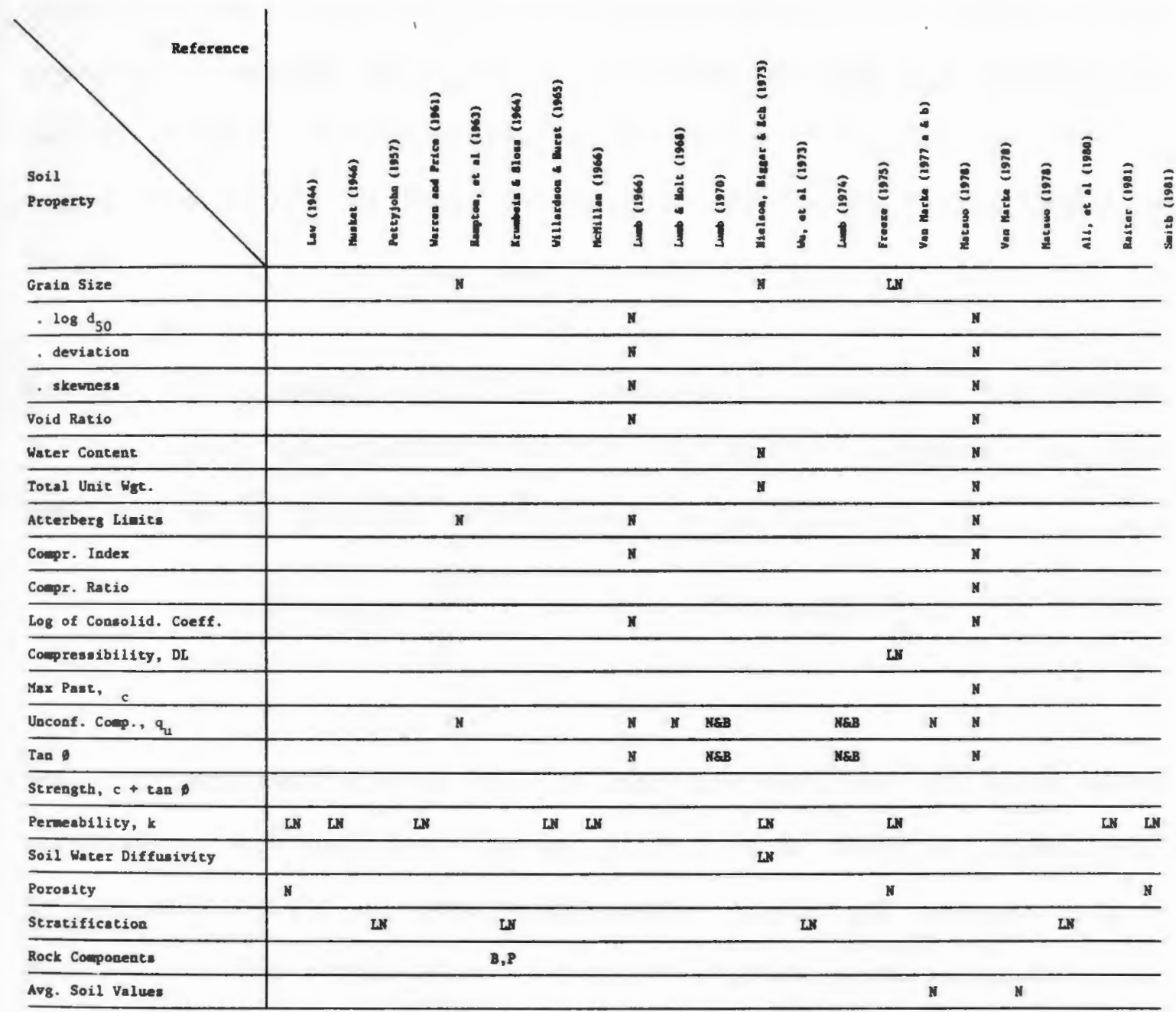

Note: $N=$ Norasl PDF, $\mathrm{LS}=$ Logaotzel PDF

$B=$ Binomial PDF, $B=$ Beta PDF

$P=P \circ i$ \#\#On PDE 


$$
\overline{\mathrm{x}}-\mathrm{z}_{\alpha / 2} \frac{\sigma_{\mathrm{x}}}{\sqrt{\mathrm{N}}}<\overline{\mathrm{x}}<\overline{\mathrm{x}}+\mathrm{z}_{\alpha / 2} \frac{\sigma_{\mathrm{x}}}{\sqrt{\mathrm{N}}}
$$

In this inequality, $\bar{x}$ is the sample mean, $\sigma_{x}$ is the population standard deviation and $Z_{\alpha / 2}$ is the value of $(\bar{x}-\bar{x})$ at which the CDF of the normal distribution equals á/2. Unfortunately, the value of the population standard deviation is not known and we must utilize the sample standard deviation as an estimate. This may not have a significant effect on these estimates, particularly if the sample is large.

However, if the sample size is not large but the underlying distribution is the normal distribution, then another similar inequality has been developed and is presented below from Miller \& Freund (1977).

$$
x-t_{\alpha / 2} \frac{s}{\sqrt{N}}<\bar{x}<\bar{x}+t_{\alpha / 2} \frac{s}{\sqrt{N}}
$$

Here the variables are the same as indicated earlier. In all of these expressions, the confidence in the prediction is directly proportional to the scatter in the population and/or sample and indirectly proportional to the sample size. Given the population variance, the confidence in the estimates may be increased by increasing the sample size.

Estimates of confidence in the standard deviation of the variable for large samples taken from normal or near normal distributions may be made by using the following in equality from Miller and Freund (1977): 


$$
\frac{\mathrm{s}}{1+\frac{\mathrm{Z}_{\alpha / 2}}{\sqrt{2 N}}}<\sigma<\frac{\mathrm{s}}{1-\frac{\mathrm{Z}_{\alpha / 2}}{\sqrt{2 N}}}
$$

In deterministic solutions to groundwater flow problems, data from field explorations and laboratory analysis are utilized to identify soil types, stratification and mean or "conservative" values for soil properties. A stochastic representation of these soil units or strata may be developed in accordance with the definitions in Greenkorn and Kessler (1969). Figure A.9 presents frequency distributions for hydraulic conductivity for the four possible combinations of uniformity and homogeneity.

Case a in Figure A.9 represents the typical deterministic representation of a soil medium. It is virtually inconceivable that a single value or even two values of hydraulic conductivity would be representative of a real soil medium although some attempts have been made in this area. We shall discuss these later. Cases a and b cannot be expected to accurately simulate real field media unless a singular value were determined. Case $c$ is an example of a singular modal probability density function such as a normal or $\log$ normal distribution which have been found to adequately represent the variability of several soil properties. This case of soil property distribution has also been referred to as statistically homogeneous and this definition will be adopted here.

The nature of the origin of a soil deposit is reflected in its flow characteristics. The number of factors which affect deposition of soil 


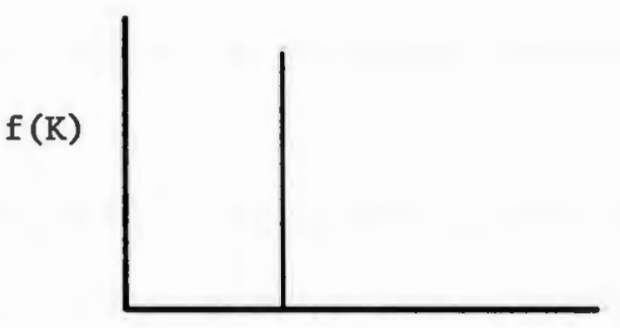

K

Uniform Homogeneous

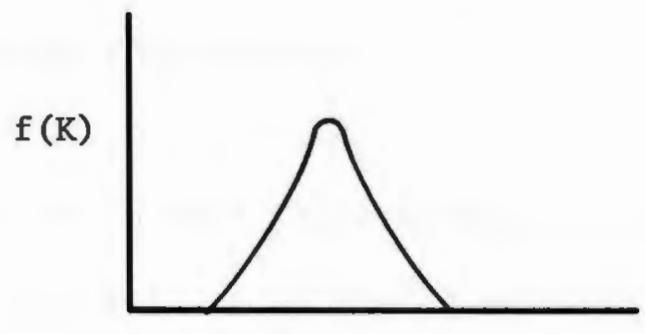

K

Nonuniform Homogeneous

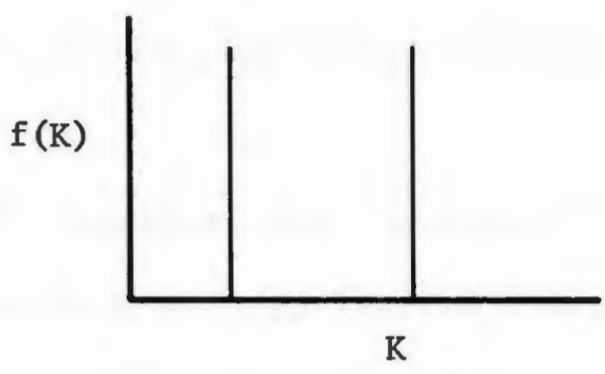

Uniform Hetergeneous

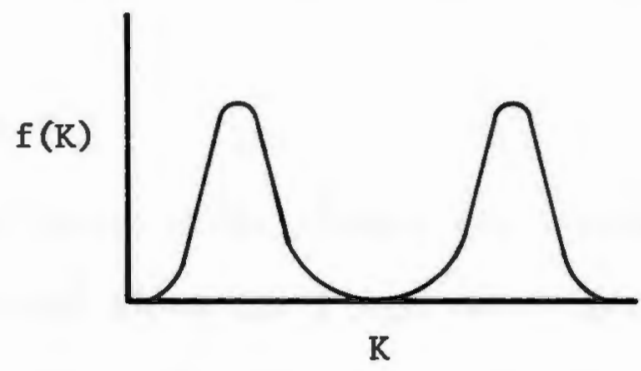

Nonuniform Hetergeneous

Figure A.9 - Possible Probability Density Functions for Hydraulic Conductivity, after Greenkorn and Kessler (1969) 
is immense and variable. Conditions predominant at one time and place will undoubtedly be somewhat altered elsewhere resulting in changes in flow characteristics. The combinations of these factors are random, but it should not be unexpected to find similarities at adjacent locations when compared to locations separated by some distance.

Lumb (1974) developed an expression for a soil property which is dependent on the value of that property on either side plus a random distortion. This expression written in terms of the random variable, hydraulic conductivity, $k$, where $a$ and $b$ are measures of dependency is given below:

$$
k_{i}=a K_{i-1}+b k_{i+1}+E_{i}
$$

This expression indicates a dependency only in one dimension, i.e., the direction of the $i$ axis. The purely random distortion is included in the term $E_{i}$ which would be a value taken from a distribution with mean of zero and a specified variance.

Lumb suggests that generally soil properties should be expected to display dependency forward and backward along the $i$ axis when this coincides with the horizontal, and some soil properties may also show dependency in one of the vertical directions.

Bartlett (1975) extended the work of Whittle (1954, 1966) on a nearest neighbor stochastic process model. This model essentially relates the value of a random variable at one point to that at adjacent points. 
Other work on the development of this model has been done by Brook (1964), Besag (1974) and Martin (1974) on agricultural forecasting.

Smith and Freeze (1979a) developed a similar equation where a and b are equal. They refer to this technique as a nearest neighbor stochastic process model. Their terminology has been adopted herein. In their companion paper, Smith and Freeze (1979b) extended this to allow treatment of a dependency in two dimensions. Their expression is similar to that given below:

$$
K_{i j}=\alpha_{x}\left(K_{i-1, j}+K_{i+1, j}\right)+\alpha_{y}\left(K_{i, j+1}+K_{i, j+1}\right)+E_{i j}
$$

In this expression, the alpha terms quantify the dependency of the hydraulic conductivity values in the $x$ and $y$ directions.

Smith \& Freeze utilized this expression to develop a series of simultaneous equations at all grid points within a specified flow region. These equations are solved for each stochastic representation, and the ensemble of equally probable distributions are statistically analyzed for significance. It is this method of Monte Carlo simulation of equally probable representations of the flow media which was selected for further investigation in this work.

A statistical measure of dependency of one random variable upon another is the correlation. The term autocorrelation refers to that dependency of the value of the random variable at adjacent locations. The autocorrection coefficient $\rho(1)$ may be defined as: 


$$
\rho(1)=\frac{\frac{1}{N}-1 \sum_{i=1}^{N-1}\left(K_{i}-\bar{K}\right)\left(k_{i+1}-\bar{K}\right)}{\frac{1}{N}-1 \sum_{i=1}^{N}\left(K_{i}-\bar{K}\right)^{2}}
$$

In this expression $K_{i}$ and $K_{i+1}$ are the value of $K$ at node $i$ and $i+1$ respectively, and $\overline{\mathrm{K}}$ is the mean value of $\mathrm{K}$. It should be noted that because we have assumed a statistically homogeneous media, the mean and variance of the adjacent $K$ values are equivalent and that the denominator equals the variance of $\mathrm{K}$.

An interesting result of this parameter is that it may vary only within the range of -1 and +1 . A value of zero indicates no dependency, that of one indicates complete dependency, and a minus one indicates an inverse relationship.

Whittle (1963) developed expressions for the autocorrelation function assuming singular direction or one dimensional dependency, i.e., a unilateral Markov Process of:

$$
\rho(1)=\exp (-\alpha 1)
$$

Figure A.10 presents a plot of an autocorrelation function which would illustrate this relationship of correlation and distance. The correlation decays with distance.

Whittle also presented a similar equation for a bilateral Markov Process where $\mathrm{K}_{1}(\mathrm{x})$ is the modified Bessel function of the second kind: 


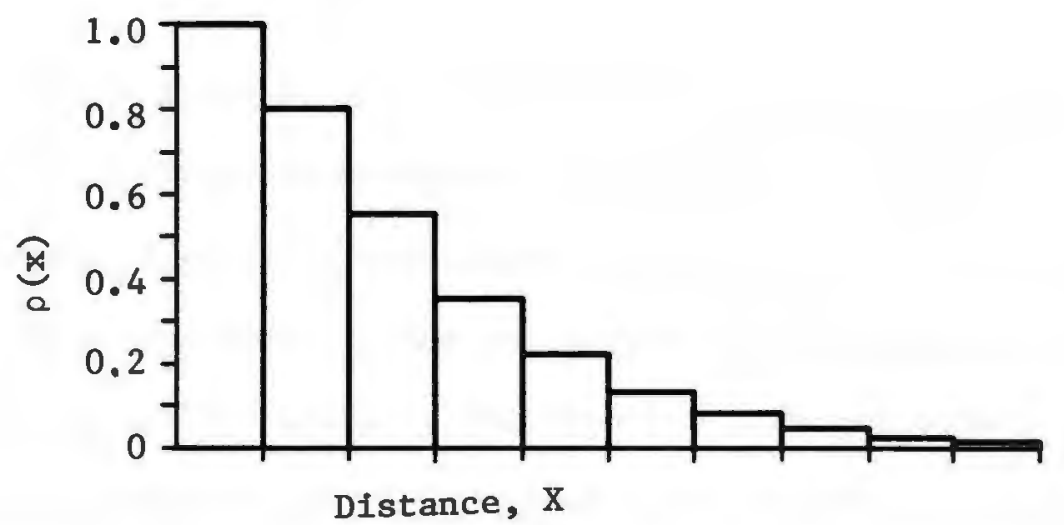

- Discrete Block Mode1 -

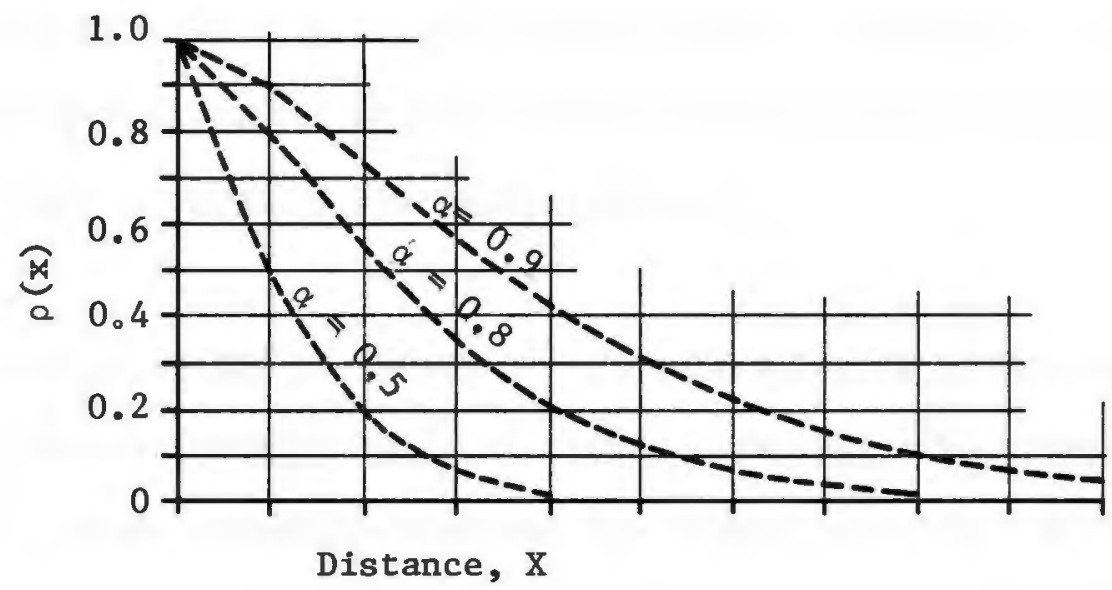

- Continuous Mode1 -

Figure A.10 - Autocorrelation Decay with Distance, after Smith and Freeze (1979) 


$$
\rho(1)=(B 1) K_{1}(B 1)
$$

Agterberg (1970) derived the expression for line sampling of a three dimensional autocorrelation which is equivalent to the one for a unilateral Markov Process given above. Agterberg indicated that this expression has been found to be in agreement with experimental autocorrelation function for residuals obtained for geologic data. Lumb (1974) compared these theoretical expressions with results of testing on two natural soils. He concluded that for those soils, the autocorrelation function decayed quite rapidly for most soil properties. The strength of the soil with depth displayed the most sustained autocorrelation function.

Lumb (1974) concluded, however, that for a spacing of 1 to 2 meters, there would be no significant error in assuming an uncorrelated pattern. He suggested that for the soils studied, that a treatment of the soil property random variable as the sum of a trend component and a random component would perhaps be the best approach.

This alternative method of treatment of the similarity of soil parameter is referred to as trend analysis or trend surfacing. The development of this technique is generally credited to Krumbein and Whitten at Northwestern University, see e.g. Krumbein (1959) and Whitten (1959). The technique is something similar to the contouring of an area indicating spatial trends of high values compared to other areas with a lower value trend. 
A form of regression equation is typically utilized to relate the unknown value of the parameter, e.g. w, at each location within the region. The value of $w$ is expressed as a function of one or more independent variables, $x_{i}$, representing coordinates, corresponding constant coefficient, and a random component, e. The random component is usually assumed to be normally distributed with a mean of zero and a standard deviation characteristic of the scatter within the soil determined in the field. Koch and Link (1970) present an excellent introduction to this technique with examples of its practical use. The simplest example of this technique is a linear one in one dimension. An equation may be written for the dependent variable $w$ as follows:

$$
\mathrm{w}=\mathrm{C}+\mathrm{Bx}+\mathrm{e}
$$

Here $B$ and $C$ are undetermined constants depending on the data. If we plot the data with the dependent variable as the ordinate and the coordinate $x$ as the abcissa, then $C$ will equal the intercept and $B$ the slope of the line. The "best fit" for this line is characteristically determined by the method of least squares.

The e term may now be evaluated from the residuals, i.e., the variation of the data points from the fitted line. It is generally assumed to have a normal distribution with a mean of zero and a variance equal to the mean value of the squared distance for the "best fit" line.

This method may get far more intricate as the parameter to be modeled requires it. Two and three dimensional models are possible. The coordinate terms may include not only the first power of these coordinates, 
but also higher powers and cross products in order that the trends be adequately mapped.

Tabba and Yong (1981a) have developed a working model for a quasi homogenous soil utilizing the trend analysis technique. They combine the bias in the parameter measurement method with the random variation component so that the mean of the random variation will no longer always be zero.

Tabba and Yong (1981b) demonstrate how this technique may be utilized to assist the solution of real problems. This technique will essentially provide a complete representation of a flow medium's parameter given the value of flow media's characteristic at certain locations.

An alternative method most frequently discussed in the literature is the spectral density treatment of the soil property random variable. Bakr (1976), Gelhar (1977), Gutjahr, et. al. (1978) and Gelhar, et. al., have demonstrated its use in groundwater flow problems. This approach recognizes the Fourier Transform pair consisting of the spectral density function for the random variable, $S(f)$, and the autocorrelation function, $R(x)$. A brief description of these concepts are presented here. A thorough presentation of this complex subject may be found in random vibration texts such as Newland (1975) and Bendat and Piersol (1971).

The soil medium is considered the result of a random process. The autocorrelation function, $R_{(x)}$, relates the influence of the value of the random variable at one point to that at some other point. The power 
spectral density function, $S(f)$, describes the general frequency information of the underlying random process. The form of the Fourier transform pair between $S(f)$ and $R(x)$ is:

$$
\begin{aligned}
& R(x)=\int_{-\infty}^{\infty} S(f) \cos 2 \pi x f(x) d f \\
& S(f)=\int_{-\infty}^{\infty} R(x) \cos 2 \pi f x d x
\end{aligned}
$$

In these expressions, $f$ is the frequency defined as the inverse of the distance, $x$, the distance between data points or the lag distance.

A random process which is large in the space domain will be small in the frequency domain. This is illustrated in Figure A.11 which presents the probability density function, autocorrelation function and power spectral density plot for a wide band random noise process.

The autocorrelation functions for the random variables discussed earlier as being characteristic of soil properties have been positive exponential functions similar to that shown on Figure A.11. Its power spectrum would therefore have a peak at low frequencies and decay fairly rapidly. Conversely, if the autocorrelation function decays rapidly, the power spectral density will shift to higher frequencies. The term white noise is referred to a random process with all frequencies of equal power and no correlation in the space domain.

This approach to the treatment of the media characteristics has a distinct advantage. Variations in soil properties may be treated in the continuum sense and therefore more exact representations of the medium are possible. The method suffers however from difficulties for the 


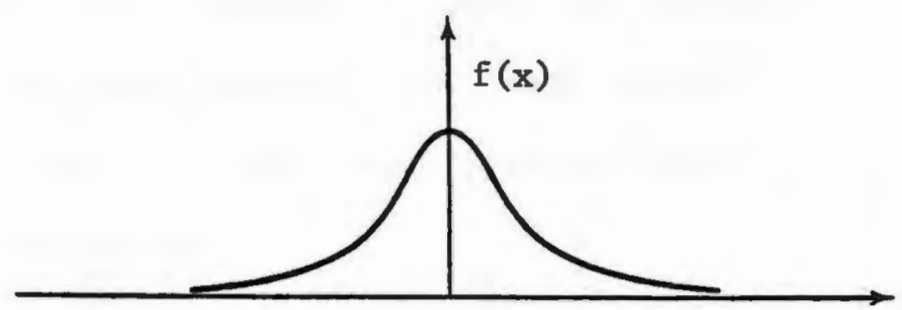

Probability Density Function
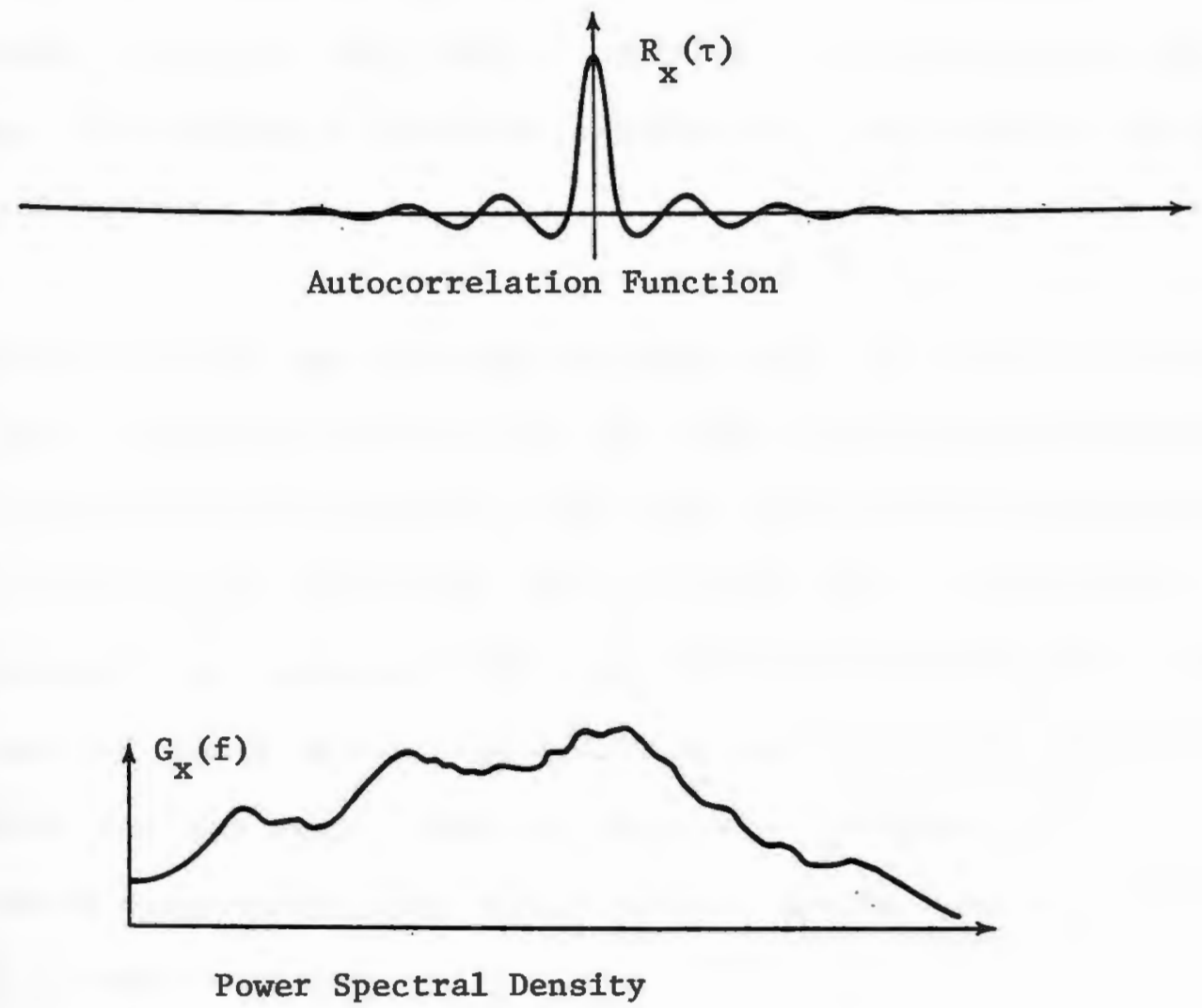

Figure A. 11 - Wide Band Random Noise Process, after Bendat and Piersol (1971) 
average practitioner in understanding the concept, as well as the large amount of real data required to make the procedures produce significantly more realistic result. The large amount of data required casts doubt on its use for most real problems due to the difficulty in verifying the parameters.

Van Marke (1977, 1978) describes the benefits of a probabilistic modeling of soil profiles. Van Marke proposed an alternate method for simulation of soil profile modeling which recognizes the variability in the soil and considers one or more of the soil properties as random variables which are functions of location. Figure A.12 presents this concept for a multi-strata representation of a soil profile in two and three dimensions.

Van Marke proposed that the soil parameter could be completely described by three statistical parameters: the mean, the standard deviation and the scale of the fluctuation. The scale of the fluctuation quantifies the distance over which the value of the soil property has strong correlation. He indicates that this scale of fluctuation is close to the mean distance between intersections of the value of the random variable and the mean. This is illustrated in Figure A.12. In fact, Van Marke demonstrated that this technique may be used to estimate the scale of the fluctuation.

Van Marke points out that this representation of the soil parameter random variable is quite similar to the approaches mentioned earlier. The scale of fluctuation is a different but related measure of correla- 


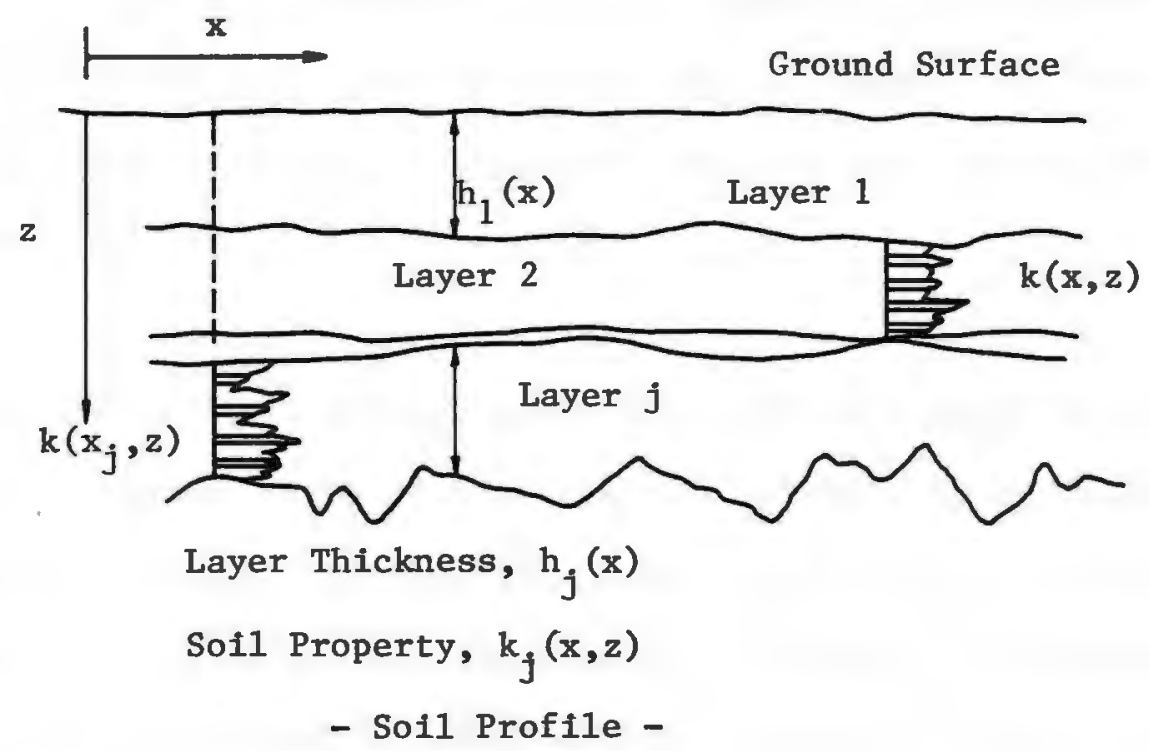

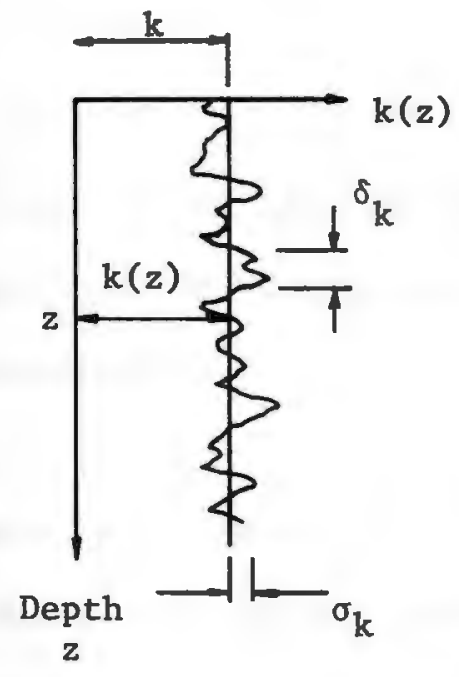

Variation of Soil Property with Depth

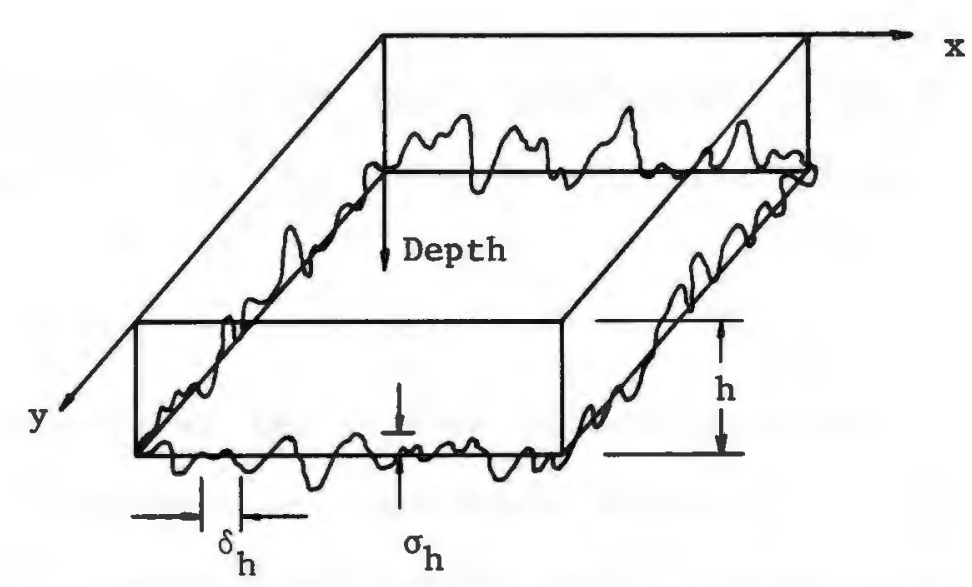

Variation of Depth to rock in $x-y$ Plane

Figure A.12 - Van Marke's Stochastic Representation of Soil Profile 
tion of soil property. The concept of developing a large number of random variables in the problem solution, e.g., numbers and thicknesses of strata and soil properties such as hydraulic conductivity, porosity and dispersivity have great potential but it seems that this diversity will be very difficult to achieve due to the computational effort required.

As indicated earlier, several researchers have attempted to determine if a single "average" value of hydraulic conductivity in a homogeneous soil could satisfactority represent the conditions in a more realistic hetergeneous soil. Warren and Price (1961) determined that the geometric mean would be the best estimate of this "average" value. The geometric mean, $\mathrm{Kg}$, is defined as:

$$
\mathrm{Kg}=\sqrt[N]{\mathrm{K}_{\mathrm{i}} \cdot \mathrm{K} 2 \cdots \mathrm{Kn}}
$$

Bouwer (1969) demonstrated this finding for a hetergeneous soil by numerical and analog methods calling this value the effective hydraulic conductivity.

Smith and Freeze (1979) summarized the results of several other researchers indicating that the geometric mean should be equal to this "effective conductivity" for steady state uniform flow. However, the geometric mean could not accurately estimate the effective hydraulic conductivity for non-uniform and/or transient flow.

Bouwer (1969) points out that the arithmetic mean is the normal average value, $K_{a}$ where 


$$
\mathrm{K}_{\mathrm{a}}=\frac{\mathrm{K}_{1}+\mathrm{K}_{2}+\cdots \mathrm{K}_{\mathrm{N}}}{\mathrm{N}}
$$

Willardson and Hurst (1965) indicate that this mean would be the best estimate if the soil were truly homogeneous and all variations were due to measurement error. Bouwer (1969) demonstrates that the arithmetic mean should be the best estimate for a highly stratified flow parallel to the layering and the harmonic mean should be the best estimate for flow perpendicular to the layering. The harmonic mean, $K_{h}$, is defined as:

$$
\mathrm{K}_{\mathrm{h}}=\frac{\mathrm{N}}{\frac{1}{\mathrm{~K}_{1}}+\frac{1}{\mathrm{~K}_{2}} \cdots \frac{1}{\mathrm{~K}_{\mathrm{N}}}}
$$

Warren and Price (1961) suggested that a shape factor should be utilized to "normalize" the calculation of the effective hydraulic conductivity $\mathrm{K}_{\mathrm{q}}$, for non-uniform and/or transient flow. This may be achieved by calculating $K_{q}$ for the flow through a homogeneous soil with the same geometry using this result to calculate a shape factor, $S$, where:

$$
S^{\prime}=\frac{K_{c}}{K_{q}}
$$

In this equation $K_{c}$ is the value of the constant hydraulic conductivity and the $K_{q}$ is the effective hydraulic conductivity for this homogeneous soil.

VII. MASS TRANSPORT SIMULATION

Mass transport in groundwater through a stochastically generated flow 
medium has been studied by various investigators, e.g., Smith and Schwartz (1980) and Gelhar, et. al. (1979). The classical development for mass transport in groundwater in a porous medium is given in the literature by various researchers. Freeze and Cherry (1979) indicate that mass transport may involve as many as six mechanisms: advection, dispersion, adsorption, chemical reaction, biological transformation and radioactive decay. The first two are present in virtually all cases while the remaining four may or may not be significant factors in the groundwater transport.

Advection is the movement of the water caused by hydraulic gradients. Dispersion is the movement of the water resulting from the spreading of the water particles due to diffusion and the natural hetergeneity at the pore scale of the flow medium.

Freeze and Cherry (1979) developed the one dimensional transport equation which includes only these first two mechanisms and it is presented below:

$$
\mathrm{D}_{\mathrm{L}} \frac{\partial^{2} \mathrm{C}}{\partial \mathrm{I}^{2}}-\mathrm{v}_{\mathrm{L}} \frac{\partial c^{\prime}}{\partial \mathrm{I}}=\frac{\partial \dot{c}}{\partial \mathrm{t}}
$$

In this equation $D_{L}$ is the dispersion coefficient and equals the sum of the diffusion coefficient and dispersivity times the mean pore fluid velocity. ' $C$ is the concentration, $l$ is the length dimension, $v_{L}$ is the mean tracer velocity, and $t$ is the time.

Figure A.13 presents the setup and results of a common laboratory test 

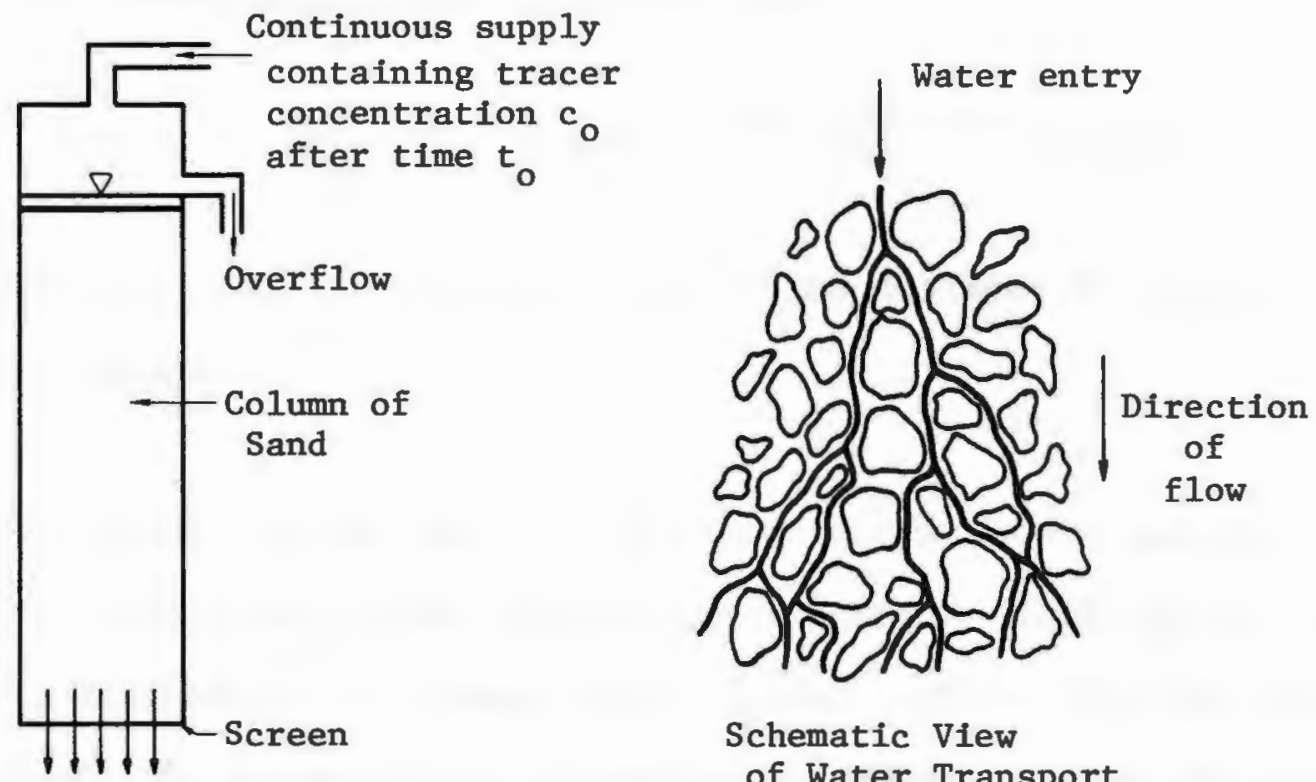

Outflow containing.

tracer concentration $c$

Schematic View of Water Transport Illustrating Dispersion

Sand Column

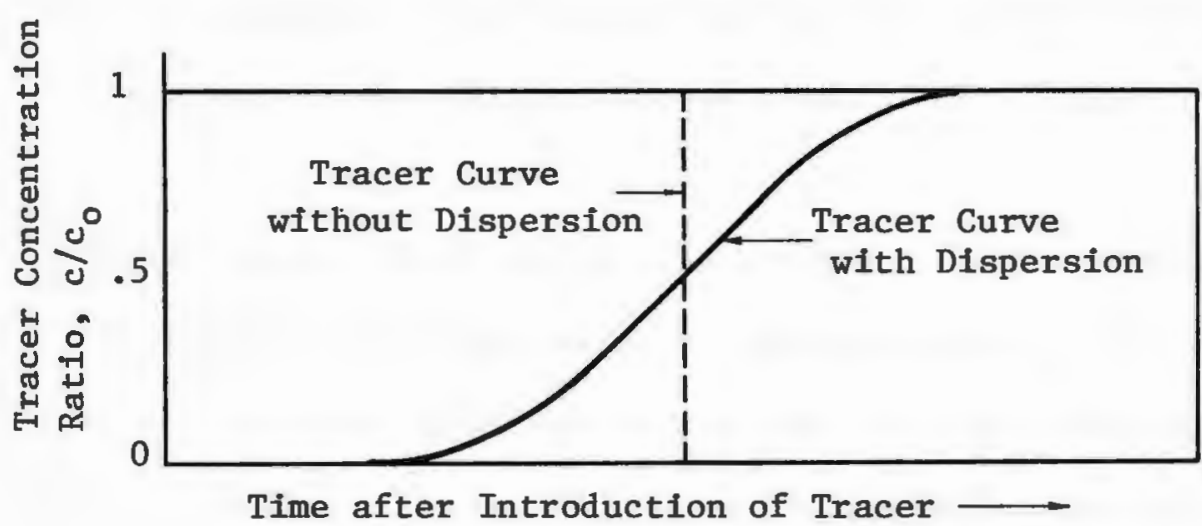

Figure A.13 - Tracer Experiment Layout, after Todd (1980) 
which may be used to demonstrate this concept. Initially, a wave front is released from the upper portal and movement and distortion is traced. Ogata (1970) presented the solution to this differential equation in terms of the complementary error function, erfc,

$$
c^{\prime} / C o=\frac{1}{2}\left[\operatorname{erfc} \frac{(1-v t)}{\left(2 \sqrt{D_{L} t}\right)}+\exp \frac{(v 1)}{(D 1)} \operatorname{erfc} \frac{(1+v t)}{\left(2 \sqrt{D_{L} t}\right)}\right]
$$

For all practical purposes the second term in the brackets is negligible and may be neglected.

The Central Limit theorem has been utilized to justify the assumption that the breakthrough curve illustrated in Figure A.13 should be normally distributed, e.g., Bear (1972), Fried (1975). This had been suggested for the displacement relationship provided the water particle is allowed to undergo a significant number of uncorrelated displacements. Fried (1975) presents a method to calculate the macroscopic or field scale dispersion coefficients given the results of a tracer transport experiment and utilizing the assumption of a normal distribution.

Smith and Schwartz (1980) utilized a nearest neighbor stochastic process model of hydraulic conductivity to characterize a uniform flow field. Water particles were released on one side and recovered along the downgradient boundary. The particles were allowed to move by hydraulically driven advection and a stochastic simulation of microscopic dispersion. Their results indicated that normality for the breakthrough curve and a constant macroscopic dispersivity could not be confirmed. 
Gelhar, et. al. (1979) studied the mass transport problem and found that for large distances the macroscopic dispersivity did appear to reach a constant value and appeared to be a function of the properties of the medium. They developed an equation for the effective macroscopic dispersivity for large times,

$$
\begin{aligned}
& \alpha_{\mathrm{LT}}=\alpha_{\mathrm{L}}+\mathrm{A}_{\infty} \\
& \text { Where } A_{\infty}=\frac{1}{3} \quad \frac{\sigma_{\mathrm{K}^{2}}}{\overline{\mathrm{K}}^{2}} \frac{\mathrm{I}^{2}}{\alpha_{\mathrm{T}}}
\end{aligned}
$$

In this equation $\bar{K}$ and $\sigma_{K}$ are the mean and standard deviation of the hydraulic conductivity, $a_{T}$ is the traverse dispersivity and 1 is the length scale.

Gelhar, et. al. (1979), concluded that the distance required to reach a constant dispersivity is large, perhaps exceeding $1 \mathrm{~km}$ to reach $90 \%$ of this value. This could explain the inability to measure a constant dispersivity on most field investigations.

A great deal of work has already been done in this area and yet we do not feel confident with the answers received by the current state-ofthe-art procedure. This work will build on the earlier work and perhaps provide some additional measure of confidence in the results computed. 


\section{APPENDIX B}

Details of Method Used

\section{B. 1 Introduction}

It has been an objective of this work to develop a procedure to more realistically characterize the medium of groundwater flow for a field problem and to arrange the output so that definitive conclusions may be drawn utilizing statistical models. It is hoped that this will result in an improvement in the confidence that an engineer may have in the predictions of flow quantity and travel times.

\section{B.2 General Technique}

In this work, a two dimensional characterization has been chosen because it is the more common and simpler representation. A three dimensional model could have been adopted, but it would have been very difficult to implement to solve any real problems because of the tremendous amount of computation required. Although the computer has made the numerical methods possible, in practice, three-dimensional solutions are still not commonly done.

A rectangular flow region was chosen for study so that a general problem might be solved. Figure B.I presents the layout of the idealized flow region with boundary conditions. Hydraulic conductivity is presumed to be statistically homogeneous with correlation in accordance with a 

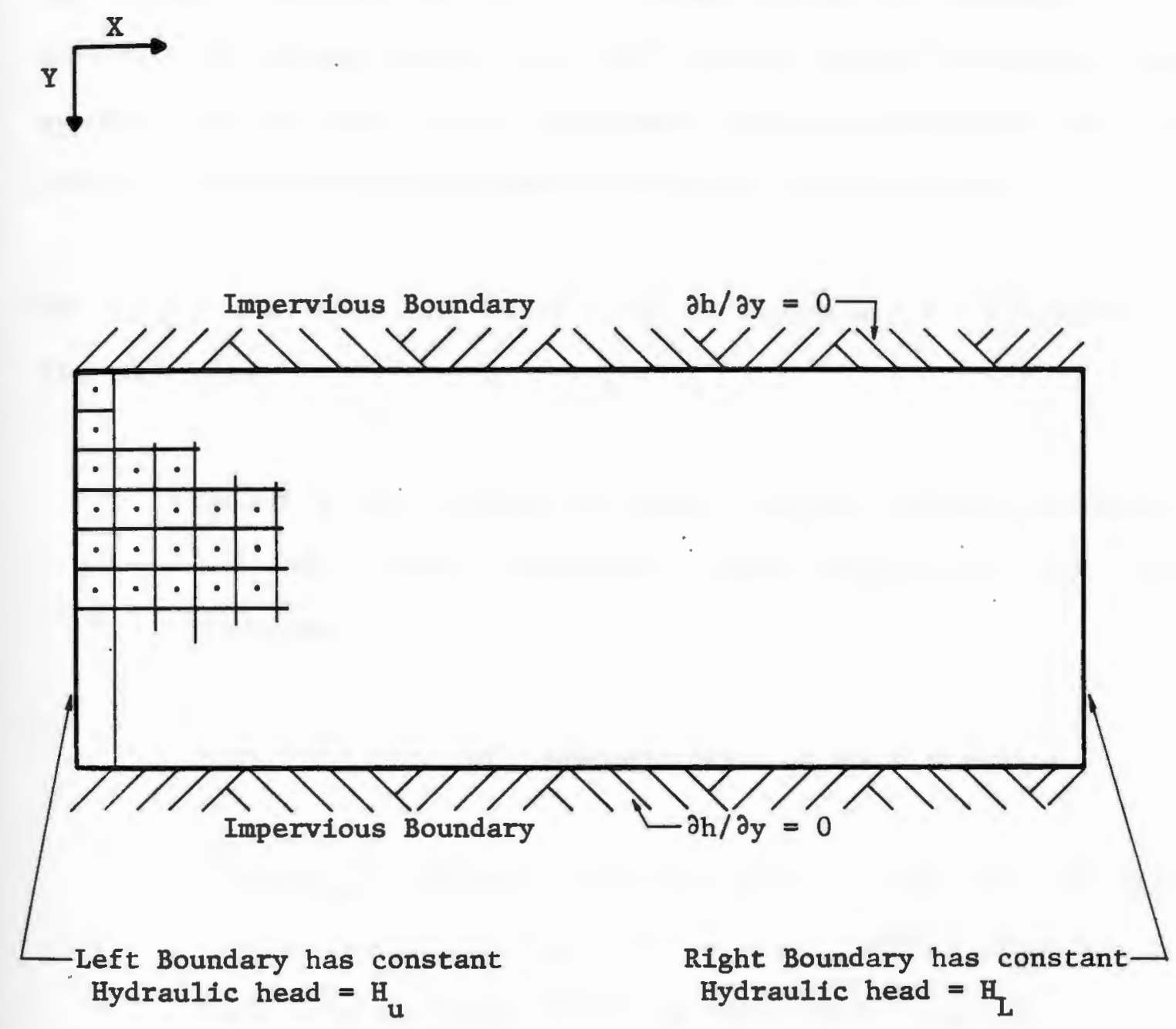

Figure B.1 - General Plan of Region 
stochastic nearest neighbor model. Variations in the soil media characterization, region shape and boundary conditions were also studied and will be discussed later.

The problem considered was one of a steady state flow situation. This condition was chosen because many real problems may be considered to be approximated by steady state conditions. The autocorrelation and dispersion techniques may be adapted to transient solutions as well.

The general procedure adopted to study this groundwater flow problem is detailed below:

1. Select a region size and shape, develop boundary conditions and soil media parameters which approximate the real situation.

2. Generate a stochastic representation of the flow media.

3. Utilizing a finite difference program with the Iterative Alternating Direct Implicit Procedure (IADI), solve for the heads and flow quantity for the steady state condition.

4. Release water particles from the upgradient side of the flow region to traverse the region under the action of advection and a stochastic simulation of dispersion. Record their respective travel times and locations upon reaching the downgradient side of the flow region. 
5. Repeat the above process until a sufficient number of Monte Carlo simulations of the region and water transport has been completed.

6. Tabularize the data and perform statistical checks of significance on output so that definitive conclusions may be drawn.

\section{B.3 Problem Definition}

The initial step requires a definition of the region size and the soil properties. This step is required in any modeling of groundwater flow whether analytical, numerical or otherwise.

An idealized representation of the region in terms of finite blocks of homogeneous soil media is required. The hydraulic conductivity, porosity and head are assumed to be constant everywhere within each finite block.

As is often the case, a good deal of experience is required to make the selection of the number of blocks to represent the region. A trade-off of accuracy against computation time is necessary for numerical modeling. Often two representations at different grid spacings are made and compared to observe the effect of the variation in grid size to aid in this decision.

\section{B. 4 Hydraulic Conductivity}


of constant hydraulic conductivity, the so-called block centered approach. Figure B.2.a. presents this diagramatically including the numbering system used. The nearest neighbor model uses the following equation to relate the conductivity at one point to all adjacent points.

$$
k_{i j}=\alpha_{x / 4}\left(k_{i, j-1}+k_{i, j+1}\right)+\alpha_{y / 4}\left(k_{i-1, j}+k_{i+1, j}\right)
$$

Where

$$
\begin{aligned}
\mathbf{K}_{\mathbf{i j}} & =\text { Hydraulic conductivity at nodal point, } \mathbf{i}, \mathbf{j} . \\
\mathbf{i} & \text { Row number with range from } 1 \text { to } \mathrm{N} \text { ROW } \\
= & \text { Column Number with range from } 1 \text { to } \mathrm{N} \text { COL } \\
\alpha_{x}, \alpha, y \quad & \text { Autoregressive parameters which indicate the degree } \\
& \text { of correlation of hydraulic conductivity in the } x \\
& \text { and } y \text { directions. Range is from } 0 \text { to } 1 .
\end{aligned}
$$

This relationship may be put in matrix form and a stochastic element introduced as follows:

$$
\{\mathrm{K}\}=[\mathrm{W}]\{\mathrm{K}\}+\{\mathrm{E}\}
$$

In this equation [W] is a weighting matrix consisting of $\mathrm{NP}$ rows and columns where NP is the total number of nodal points within the flow region, i.e., NP $=$ NROW * NCOL. The [W] matrix is square while $\{\mathrm{K}\}$ and $\{E\}$ are column matrices.

The $\{E\}$ matrix is a column matrix of random numbers with a preselected mean and standard deviation. The basis for selecting the mean and standard deviation will be discussed later. 


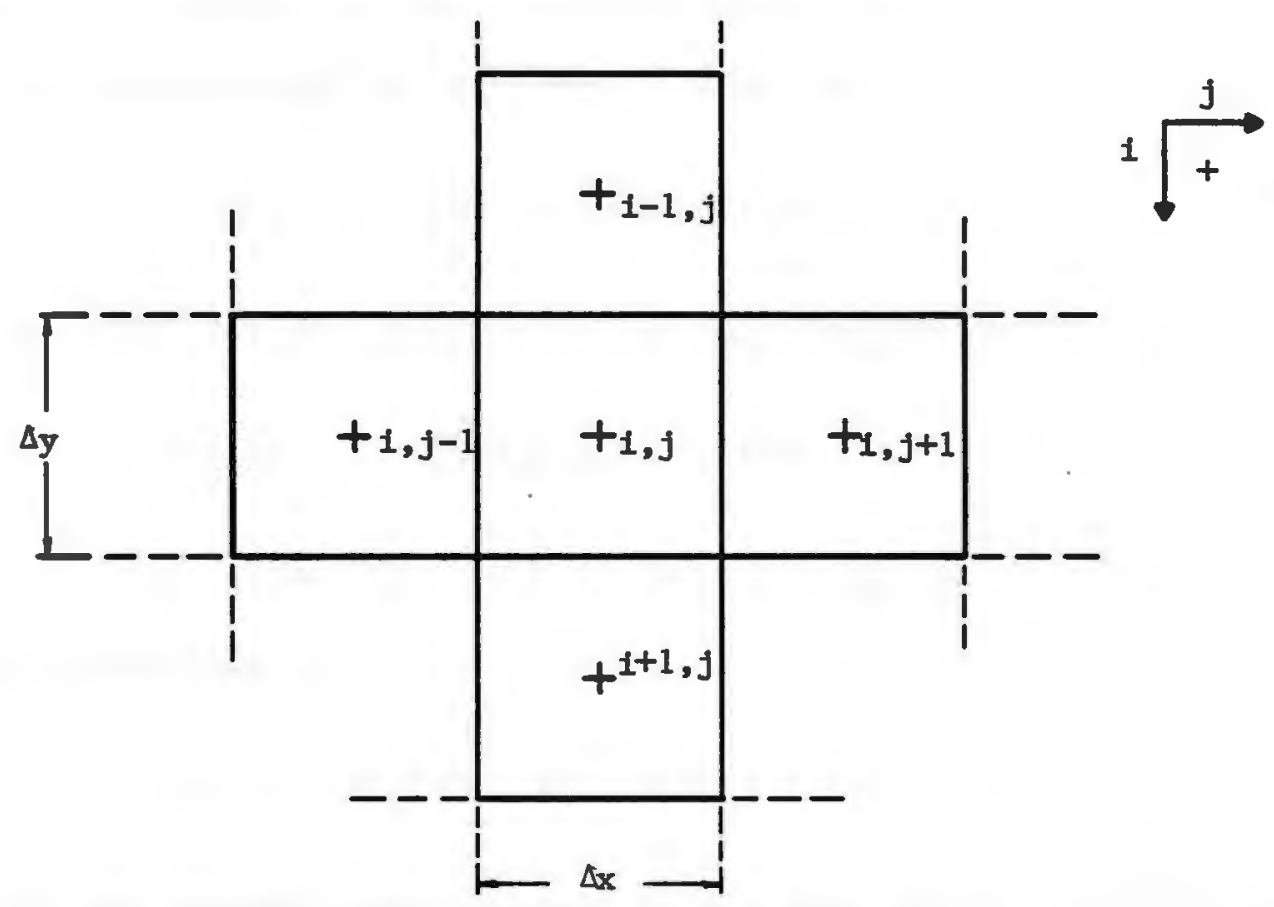

$$
\begin{aligned}
k_{i j}=a_{x} / 4\left(k_{i, j-1}+k_{i, j+1}\right)+\alpha_{y} / 4\left(k_{i-1, j}+k_{i+1, j}\right) \\
- \text { Interior Node - }
\end{aligned}
$$

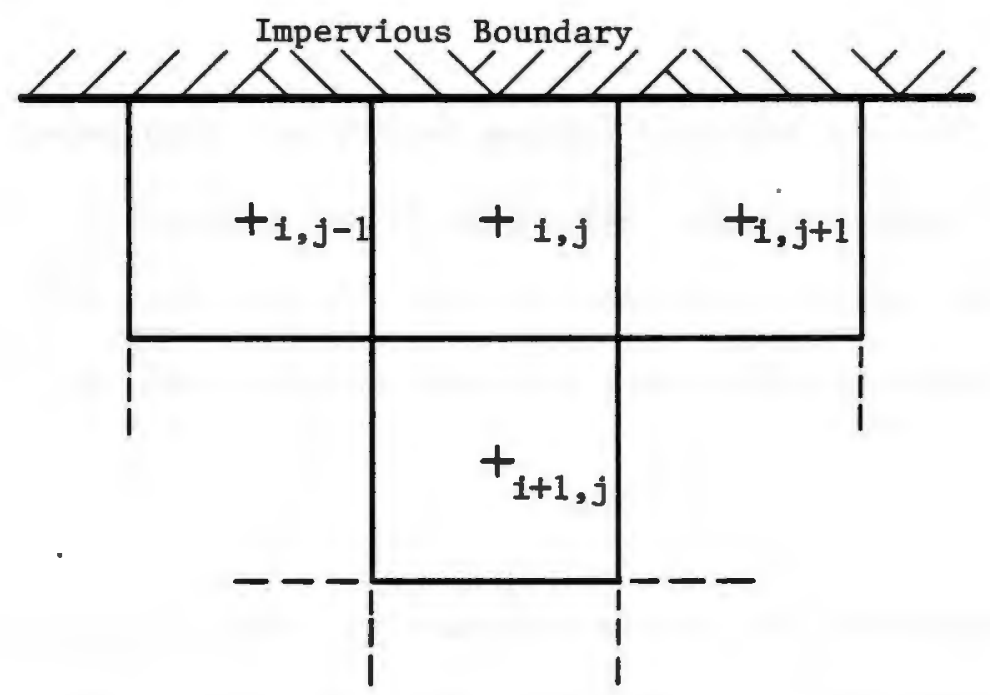

$$
\begin{gathered}
K_{i, j}=a_{x} / 3\left(K_{i, j-1}+K_{i, j+1}\right)+\alpha_{y} / 3\left(k_{i+1, j}\right) \\
- \text { Boindary Node - }
\end{gathered}
$$

Figure B.2 - Finite Difference Representation 
Since the $\{K\}$ matrix is the unknown result, we can rearrange this equation by bringing both $\{K\}$ matrices to the left side as below.

$$
\{K\}-[W]\{K\}=\cdot\{E\}
$$

We may then utilize the properties of an identity matrix and write

$$
([I]-[W])\{K\}=\{E\}
$$

Finally, we may solve for $\{k\}$ by multiplying by the inverse of $([I]-[W])$ resulting in

$$
\{K\}=(I-W)^{-1} \cdot\{E\}
$$

We now have the necessary matrix equation for this method. We need only develop the Weighting matrix [W], subtract it from the identity matrix, [I], compute an inverse, which we will call the Filter matrix, and multiply by the $\{\mathrm{E}\}$ matrix.

It should be noted that the Filter matrix contains all the information concerning the autocorrelation of hydraulic conductivities with each of the blocks in the flow region. The $\{\mathrm{E}\}$ matrix contains the randomness component which is incorporated into the system of hydraulic conductivity values.

The nearest neighbor model is easily adapted for developing the [W] matrix for interior nodes. However, boundary nodes are another matter. Smith (1978) assumed that the a term is divided by the number of adjacent nodes. Figure B.2.b. presents the situation along the upper boundary. Here this approach results in the following equation: 


$$
k_{i j}=\frac{a_{x}}{3}\left(k_{i, j-1}+k_{i, j+1}\right)+\frac{a_{y}}{3}\left(k_{i+1, j}\right)
$$

An alternative treatment of these boundary nodes was considered. This alternative approach treats these areas in the same way as finite difference grid manipulations at the boundaries resulting in the following equation.

$$
K_{i j}=\frac{a_{x}}{4}\left(k_{i, j-1}+k_{i, j+1}\right)+\frac{a_{y}}{2}\left(K_{i+1, j}\right)
$$

This approach results in greater weight being assigned to the $K$ value horizontally along a soil layer for the end boundaries and less along the upper and lower boundaries. There would be no difference in the equations written at the corners of the flow region. Comparison of results computed by each treatment will be discussed later.

Figure B. 3 presents a 7 by 7 flow region and the numbering system adopted. Since there is a maximum of 4 nearest neighbors, there is a maximum of 4 non-zero entries in each row of the matrix. The [W] matrix which results with this numbering system is banded. A band width equal to two times the number of columns plus one is centered on the diagonal. The matrix is, however, not symmetrical. Figure B.4 presents the W matrix for the 7 by 7 flow region shown utilizing Smith's boundary conditions.

A log normal distribution of hydraulic conductivity is adopted here. Appendix A discusses the background for this assumption. As a consequence of this assumption, the $\{E\}$ matrix generated for each simula- 

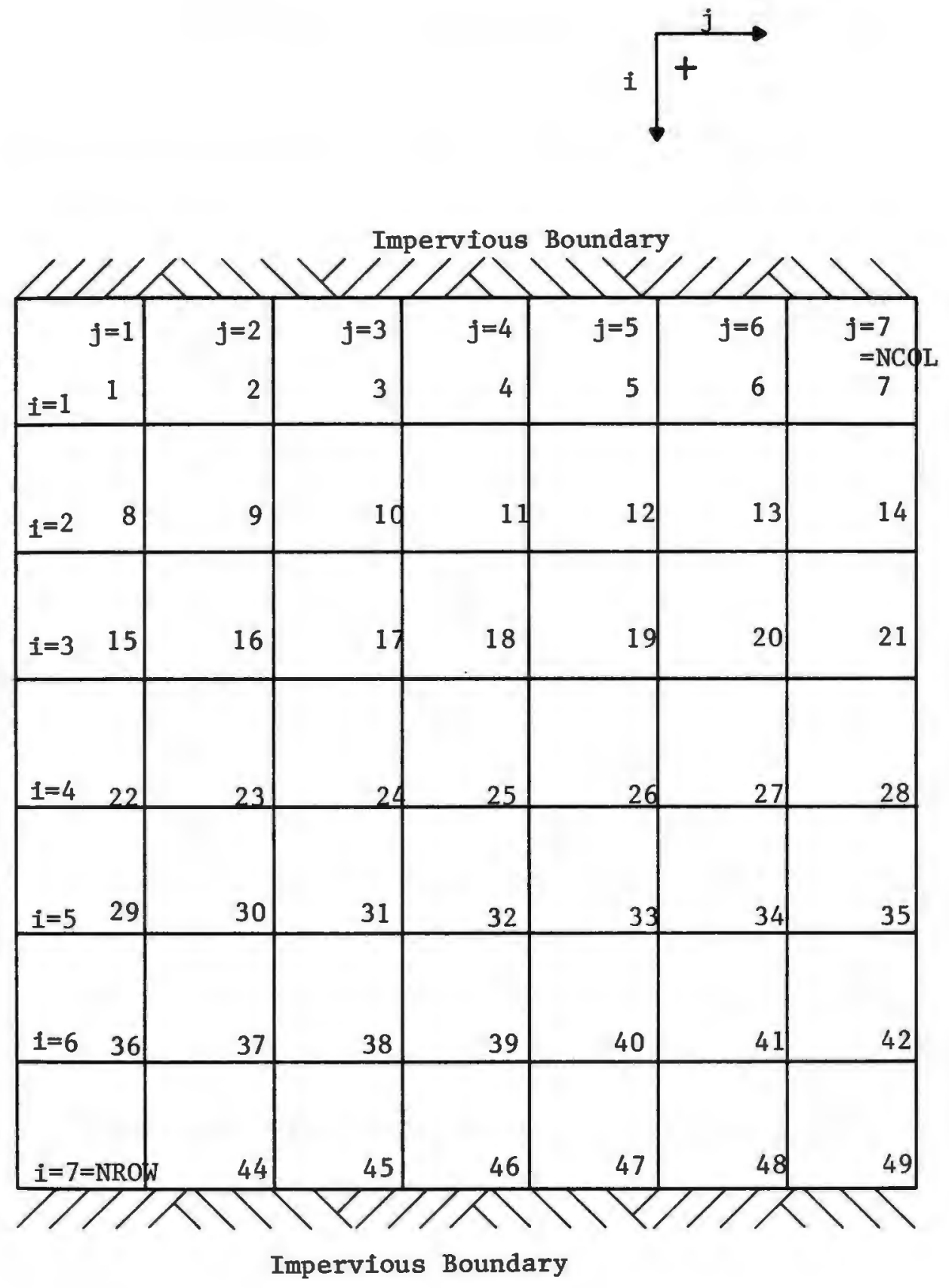

$\mathrm{NP}=\mathrm{NROW} * \mathrm{NCOL}=7 * 7=49$

Figure B.3 - 7 By 7 Example of Region Representation 
Note: All spaces in matrix not occupled by a letter or zero should contain a zero.

See the Legend below for explanation of letter designation.

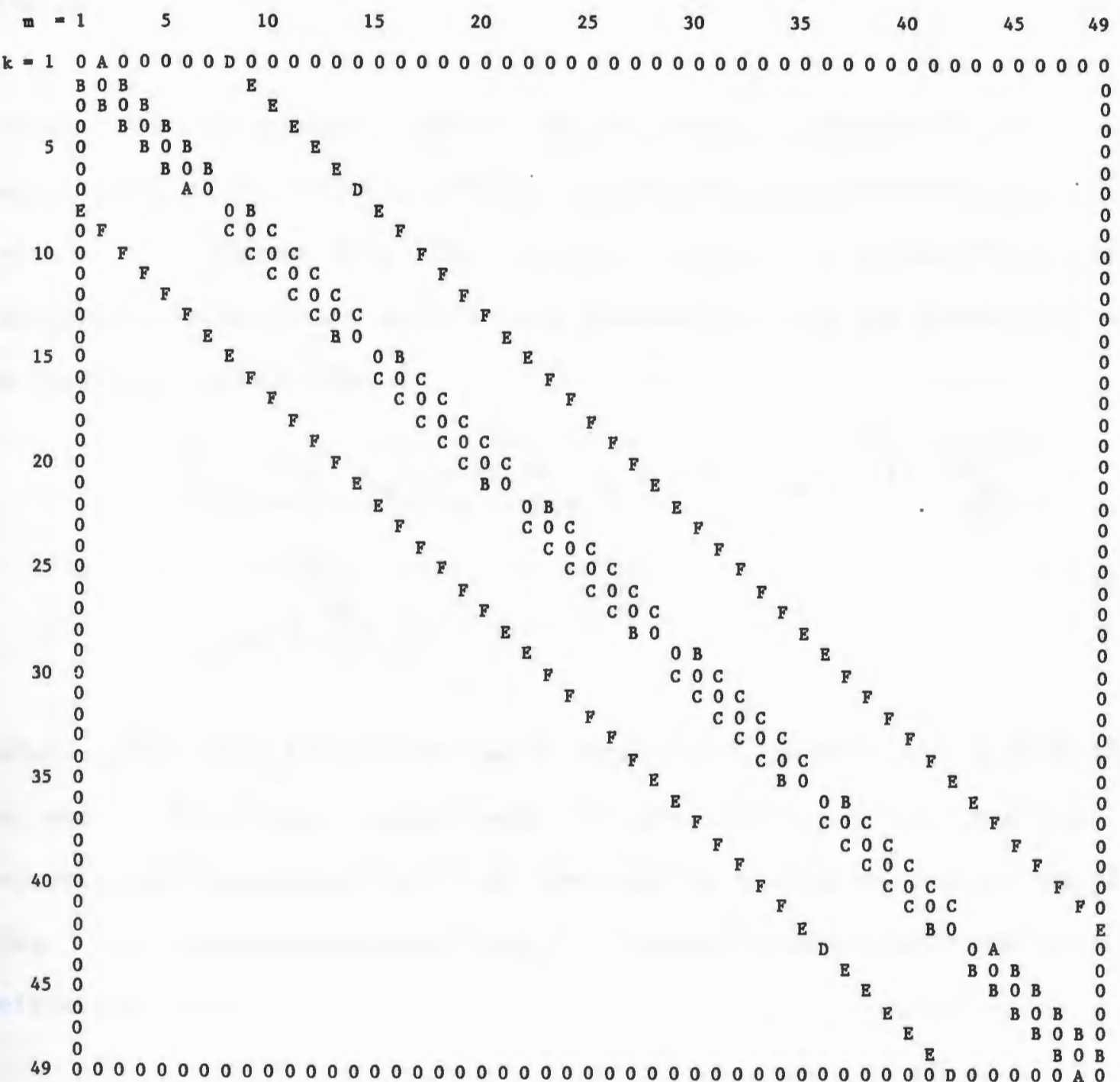

LEGEND :
$A=\alpha_{x} / 2$
$D=a_{y} / 2$
$B=\alpha_{x} / 3$
$E=\alpha_{y} / 3$
$C=\alpha_{x} / 4$
$F=a_{y} / 4$

Figure B.4 - Weighting Matrix for 7 By 7 Region 
tion will have a mean value of 0 , a standard deviation of 1 and will follow a normal distribution. A log transformation will later be made so that the hydraulic conductivity values will be $10 \mathrm{~g}$ normally distributed.

Smith (1978) demonstrated that if the $\{E\}$ matrix is multiplied by a constant term, $n$, the desired variance in the hydraulic conductivity values results. He showed that this constant term, $n$, is a function of the desired variance of the conductivity distribution and the square root of a quantity $\mathrm{G}$ defined as:

$$
\begin{aligned}
G= & \frac{\alpha^{2}{ }^{2} y(2)}{8}+\frac{\alpha x^{\alpha} y^{\rho(1,1)}}{2}+{ }^{\alpha} y^{\rho}{ }^{(1)}-{ }^{\alpha} x^{\rho} x^{(1)}+\frac{\alpha y^{2}}{8} \\
& +\frac{\alpha x^{2}}{8}+1+\frac{\alpha^{2} \rho x^{(2)}}{8}
\end{aligned}
$$

where $\rho_{X}(1)$ and $\rho_{y}(1)$ are the $\operatorname{lag} 1$ correlation coefficients of $K$ in the $x$ and $y$ directions respectively. $\rho_{x}(2)$ and $\rho_{y}(2)$ are the $\operatorname{lag} 2$ correlation coefficients of $K$ in the $x$ and $y$ directions. $\rho(1,1)$ is the $\operatorname{lag} 1$ in the $x$ direction and $\operatorname{lag} 1$ in the $y$ direction correlation coefficients of $K$.

Because of the assumption of a $10 \mathrm{~g}$ normal distribution of hydraulic conductivity, a transformation of the mean and standard deviation is required. It should be pointed out that the mean of the distribution of In $K$ does not equal the natural log of the mean of $K$. Benjamin and Cornell (1970) among others, present this transformation. 


$$
\begin{aligned}
\sigma_{\mathrm{y}}^{2} & =\ln \left(\left(\sigma_{\mathrm{k}} / \overline{\mathrm{k}}\right)^{2}+1\right) \\
\overline{\mathrm{Y}} & =\ln (\overline{\mathrm{K}})-\sigma_{\mathrm{y}}{ }^{2} / 2
\end{aligned}
$$

In these equations

$$
\begin{aligned}
\sigma_{\mathrm{y}}^{2} & =\text { variance of } \mathrm{Y} \\
\overline{\mathrm{Y}} & =\text { mean of } \mathrm{Y} \\
\sigma_{\mathrm{K}} & =\text { variance of } \mathrm{K} \\
\overline{\mathrm{K}} & =\text { mean of } \mathrm{K} \\
\mathrm{Y} & =\ln \mathrm{K}
\end{aligned}
$$

This transformation is performed and the values $\sigma_{\mathrm{y}}$ and $\overline{\mathrm{Y}}$ used with the $\{\mathrm{E}\}$ matrix. Following the multiplication by the filter matrix and addition of the mean of the ln $K$, a transformation back to $K$ is made. This transformation erodes some of the correlation although this has not been found to be significant (Smith (1978)).

This method does not produce a distribution of hydraulic conductivity which fits exactly the desired correlation structure. This is due to the randomness inserted by the random number generator, the exponential transformation and boundary effects. However each generated distribution is correlated and approximates the desired correlation structure. When the ensemble of all Monte Carlo simulations is studied, a closer approximation to the desired correlation is obtained.

The computer code developed to perform these operations referred to as Inverse is included in Appendix D. A step-by-step description of the procedure is given below. 
1. All data on the flow region characteristics are read into the computer and problem setup. (Iines 340 to 2320)

2. The transformation of statistical parameters for $K$ to $\ln K$ is performed. (Lines 2330 to 2370)

3. The $n$ term, (ETAN), is set equal to the required percentage of the standard deviation of ln K. (Lines 2372 to 2373)

4. A column matrix (EPSILN) of random numbers which are normally distributed with a mean of 0 and standard deviation of $\sigma_{y}$ is generated, $N\left(0, \sigma_{Y}\right) \cdot$ (Lines 2375 to 2410$)$

5. The square ([I] - [W]) matrix is generated directly by inserting a 1 on the diagonal and making all other non-zero coefficients of the [W] matrix negative. (Lines 2420 to 3300)

6. The ([I] - [W]) matrix is then inverted and the new matrix, FILTR, stored on permanent disk file. (Lines 3310 to 3370 )

7. The FILTR matrix is then multiplied by the EPSILN column matrix yielding the LNK matrix having $N\left(0, \sigma_{\mathbf{y}}\right)$. (Lines 3380 to 3440 )

8. The desired mean value of $\mathrm{ln} K$ is then added to each term resulting in the XINK matrix with $N\left(\bar{Y}, \sigma_{y}\right)$. (Lines 3450 to 3510) 
9. The transformation is then made back to $\{K\}$ by taking the exponential of each term in the XLNK matrix and assigning the resulting $K$ value to its respective position in the flow region. The resulting $\{\mathrm{K}\}$ matrix has a $\log$ normal distribution with the desired statistical parameters within allowable tolerances. (Lines 3520 to 3530)

10. A check is then made for these statistical parameters, i.e., mean, standard deviation and auto correlation by rows, columns and the entire distribution and writes out results. (Lines 3540 to 6020 )

This hydraulic conductivity matrix, $\{K\}$, is then the input matrix used to solve the flow problem. For subsequent simulations the steps, 1 through 3 and 5 and 6 need not be completed again. Only steps 4 and 7 through 10 are needed for independent values of stochastically generated hydraulic conductivity values for subsequent Monte Carlo simulations.

It should be noted that this computer code utilizes selected subroutines from the International Mathematics and Statistical Libraries (IMSL). These subroutines perform necessary tasks for which new subroutines could have been developed. It is considered unnecessary to do that, however, when efficient, documented routines are available.

An example of the use of such a routine may be found in step 4. The EPSILN matrix is generated by invoking the GGNQF command. This step generates a random number from a normal distribution with a mean of zero 
and a standard deviation of one. This procedure is done within a DO loop as many times as necessary to assign a value at each node.

A second example of the use of the IMSL library may be found in Step 6 . The subroutine LGINF is called to invert the (I-W) matrix and thereby determine the FILTR Matrix.

The IMSL library has several routines which may be utilized to invert matrices. LGINF was selected because it is a generalized solution which could easily handle the problem. Other more efficient and/or accurate inversion routines could have been utilized to reduce required computer time or increase the accuracy of the solution. The reduction of required computer time to invert the matrix was not a major objective of this study although some efficiency might be desirable for subsequent work. The results cited later will demonstrate that the accuracy of the solution is sufficient.

Considering the difficulty at the boundary of maintaining the autocorrelation structure, it was decided to "shave off" the outer boundary nodes all around the block region. A $7 \times 7$ region would have the outer nodes deleted and thereby be reduced to a 5 x 5 region. This technique reduces the boundary effects but also reduces the size of the region which may be considered by this technique in light of the matrix inversion limitations.

To alleviate this limitation, the flow region can be considered a composite of two or more "blocks" that contain statistically homogeneous and 
internally correlated hydraulic conductivity meshes. The shaved 5 x 5 region may be systematically added to 3 other statistically equivalent regions to develop in a $10 \times 20$ region. We will see that the computer time to add these regions together is much less than that required for inverting a larger matrix. Indeed there are real limits to the size of the matrix that may realistically be inverted.

\section{B.5 Numerical Solution of Finite Difference Technique}

The finite difference solution utilized to solve the flow problem was that computer code developed by Reiter (1981). This code was adapted from other available work, e.g. Trescott, et. al. (1976) for the two dimensional cross-sectional model.

The initial task to complete in the numerical solution is to write the finite difference equation at each nodal point as described in Appendix A. Several techniques may then be utilized to find the solution, e.g., iterative and simultaneous solution of the equations. Prickett and Lonniquest (1971) and Wang and Anderson (1982) describe some of these techniques. Iterative solutions include Jacobi and GaussSeidel iteration, successive overrelaxation and the iterative alternating direction implicit. This work utilized the iterative alternating direction implicit because it was a code which was readily available and earlier work revealed that solutions could be achieved somewhat faster than for example successive over relaxation.

An explicit finite difference solution technique is a forward difference 
approximation since the head value which is being calculated is assumed a function of the previous estimate at adjacent nodes. An implicit solution involves a backward difference approximation where the head value is assumed a function of previous estimates at the node being calculated as well as at adjacent nodes. These methods were developed for transient problems but are equally applicable to steady state problems.

The IADI technique processes the equations row by row initially holding head values in adjacent rows constant. Upon completion of one pass, the same equations are processed column by column holding head values in adjacent columns constant. This completes an iteration and iterations continue until the maximum error at any point in the region is less than the requested allowable value.

\section{B.6 Mass Transport}

A major objective of this study was to analyze the effects of hydraulic conductivity variations on dispersion. A computer code was developed to simulate transport across the hydraulic field resulting from a steady state solution. Transport occurs due to hydraulic advection and a stochastic simulation of microscopic dispersion.

\section{B.6.1 Advective Transport}

The methodology utilized to determine the hydraulic head distribution has been described earlier. The results of each solution of a stoch- 
astically generated realization of hydraulic conductivity is a steady state flow field. These results provide all the necessary input data to simulate advective transport.

As cited in Appendix A, the variation in porosity is much less than the variation in hydraulic conductivity. Porosity has been assumed constant throughout the region similar to the assumption in Gelhar, et. al. (1979) and Smith \& Schwartz (1980).

The solution assumes that hydraulic head, hydraulic conductivity, porosity and therefore the resulting velocity is constant throughout each individual block in the flow region. Velocities are calculated initially for each conductivity simulation and for each block within the region by the method suggested by Sauty (1976). Figure B.5 presents a representation of a portion of the flow region and the finite difference expression utilized to solve for the velocities in the $X$ and $Y$ directions. The method is basically a central difference approximation over the three neighboring nodes.

In these finite difference equations and in the figure, $v_{x}$ and $v_{y}$ are the velocities in the $X$ and $Y$ directions, respectively. The subscripts on $K$ indicate the two nodes included in the calculation of harmonic mean, e.g., $K_{10}$ is the harmonic mean of $K$ values at nodes 1 and 0 . The value $H$ is the hydraulic head at the node, $n$ is the porosity and DELTAX and DELTAY are the node spacing in the $X$ and $Y$ directions, respectively.

This equation for the velocities has to be modified for boundary blocks 


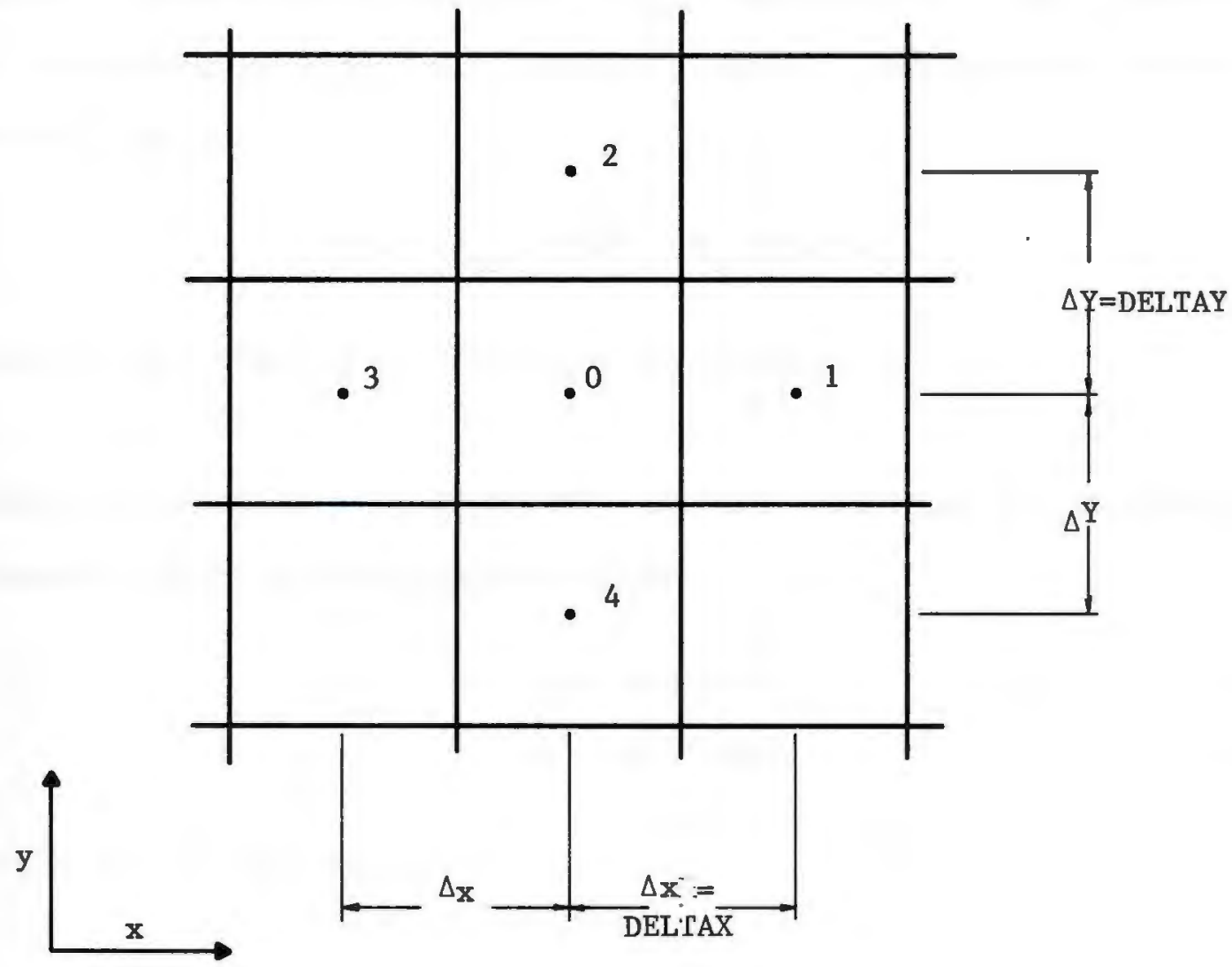

$$
\begin{aligned}
& \mathrm{v}_{\mathrm{x}}=-1 / 2 \mathrm{n}\left(\mathrm{K}_{01} *\left(\mathrm{H}_{1}-\mathrm{H}_{0} / \Delta \mathrm{x}\right)+\mathrm{K}_{03} *\left(\mathrm{H}_{0}-\mathrm{H}_{3}\right) / \Delta \mathrm{x}\right) \\
& \mathrm{v}_{\mathrm{y}}=-1 / 2 \mathrm{n}\left(\mathrm{K}_{02} *\left(\mathrm{H}_{2}-\mathrm{H}_{0} / \Delta \mathrm{y}\right)+\mathrm{K}_{04} *\left(\mathrm{H}_{0}-\mathrm{H}_{4}\right) / \Delta \mathrm{y}\right)
\end{aligned}
$$

Figure B.5 - Schematic View of Interior Node of Finite Difference Mesh 
since the velocity orthogonal to the boundary is zero. In these boundary blocks, the gradient is averaged over only one block length. An example is the upper boundary block where $\mathrm{dh} / \mathrm{dy}=0$ as in Figure B.5, which is simulated by setting $\mathrm{H}_{0}=\mathrm{H}_{2}$. The velocity in the $\mathrm{X}$ direction is calculated as before but in the $Y$ direction, the equation reduces to the following:

$$
V y=-K_{04} / n\left(\left(H_{0}-H_{4}\right) / D E L T A Y\right)
$$

Similar equations are written for other boundary blocks.

The advective transport SX and SY, is then calculated for a given time interva1, DELT, by the equations below:

$$
\begin{aligned}
& S X=V X * D E L T \\
& S Y=V Y * D E L T
\end{aligned}
$$

\section{B.6.2 Microscopic Dispersion}

Microscopic dispersion is included in the model in a manner first described by Ahlstrom, et al (1977) and then modified in part by Smith and Schwartz (1980). In this method, dispersion is treated as a stochastic phenomenon. The water particle is assumed to undergo a random displacement in the direction of flow and another random displacement perpendicular to flow. These displacements are calculated from the following two equations from Ahlstrom, et al (1977).

$$
\begin{aligned}
& \mathrm{DL}=(24 \mathrm{DLC} * \mathrm{DELT})^{\frac{1}{2}}(0.5-\text { RANOL }) \\
& \mathrm{DT}=(24 \mathrm{DTC} * \mathrm{DELT})^{\frac{3}{2}}(0.5-\text { RANOT })
\end{aligned}
$$


In these equations, DL and DT are the displacements in the longitudinal and transverse directions, respectively relative to the direction of advective transport. DLC and DTC are the longitudinal and transverse dispersion coefficients respectively. Anisotropy, if any, is presumed in the advective transport so no anisotropy is assumed in dispersivity. RANOL and RANOT are random numbers normally distributed with a mean of zero and a standard deviation of one then divided by six to keep the range of values in the -0.5 to +0.5 area.

Kelly (1982) developed a relationship which has been adopted for this study and assumes a direct relationship between grain size and microscopic dispersivity. This relationship may be expressed as:

$$
\begin{aligned}
\delta & =0.08 \mathrm{D}_{50} \\
\text { Where } \delta & =\text { dispersivity, in meters } \\
\mathrm{D}_{50} & =\text { mean particle size in millimeters }
\end{aligned}
$$

The longitudinal dispersion coefficient, DLC, is taken equal to the dispersivity times the velocity, $V_{L}$, in the direction of flow. The transverse dispersion coefficient, DTC, is taken equal to a percentage of the dispersivity, DSPRAT, times the dispersivity and velocity, $\mathrm{V}_{\mathrm{L}}$.

The expressions from Ahlstrom, et al (1977) for microscopic dispersion are then modified by these assumptions to result in the following:

$$
\begin{aligned}
& \mathrm{DL}=\left(6.3 * \mathrm{D}_{50} * \mathrm{~V}_{\mathrm{L}} * \mathrm{DELT}\right) * \text { RANOL } \\
& \mathrm{DT}=\left(6.3 * \mathrm{D}_{50} * \mathrm{~V}_{\mathrm{L}} * \mathrm{DELT}\right) * \text { DSPRAT } * \text { RANOT }
\end{aligned}
$$

Prickett and Lonniquest (1981) developed a similar approach which pre- 
sumes a constant value for DELT, the time interval.

Since the advective transport direction will not always coincide with the $X$ and $Y$ axes the displacements in the $X$ and $Y$ directions are obtained by a transformation, ie,

$$
\text { THETA }=\text { ARCTAN }(S Y / S X)
$$

The transformation is computed such that DSX and DSY are the dispersive displacements in the $\mathrm{X}$ and $\mathrm{Y}$ directions, respectively. Figure B.6 presents the vector diagram illustrating this transformation:

$$
\begin{aligned}
& \mathrm{DSX}=\mathrm{DL} * \operatorname{cOS}(\text { THETA })-\mathrm{DT} * \mathrm{SIN} \text { (THETA) } \\
& \mathrm{DSY}=\mathrm{DL} * \mathrm{SIN}(\text { THETA })+\mathrm{DT} * \operatorname{COS} \text { (THETA) }
\end{aligned}
$$

\section{B.6.3 Particle Transport}

The computer code simulates water movement from the upgradient side of the block or region to the downgradient side. Figure B.7 presents a typical problem flow region with flow from left to right. The manner in which displacements are included in the particle movement simulation within the region can now be detailed. Initially, a particle is released from the upgradient boundary. It moves a distance equal to the advective displacements, SX and SY. The random dispersive displacements, DSX and DSY are then added and the total displacements determined. A check is then made to see if the particle has moved out of the block. If is has not, a new time interval begins, new displace- 

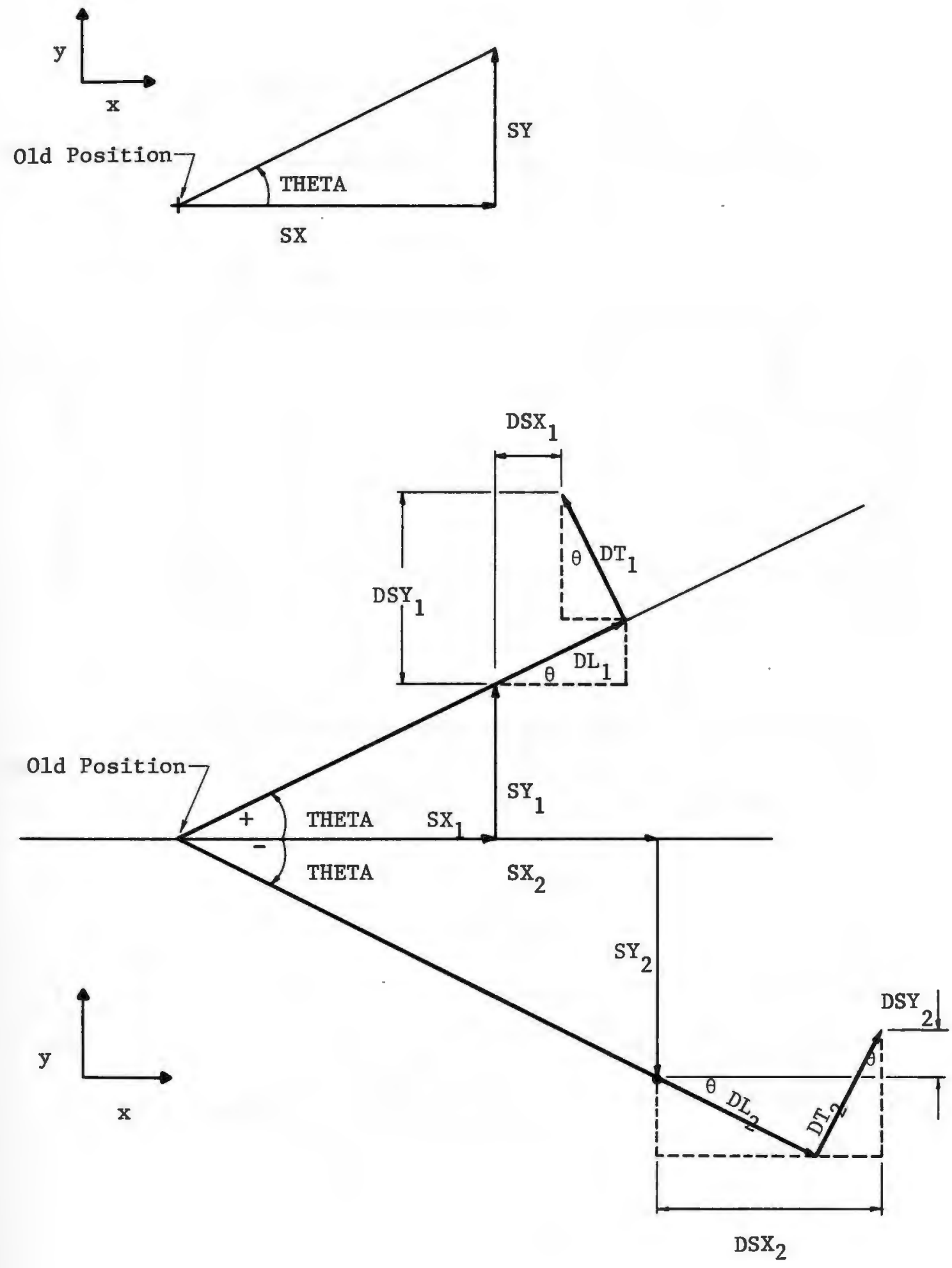

Figure B.6 - Velocity Vector Diagram 

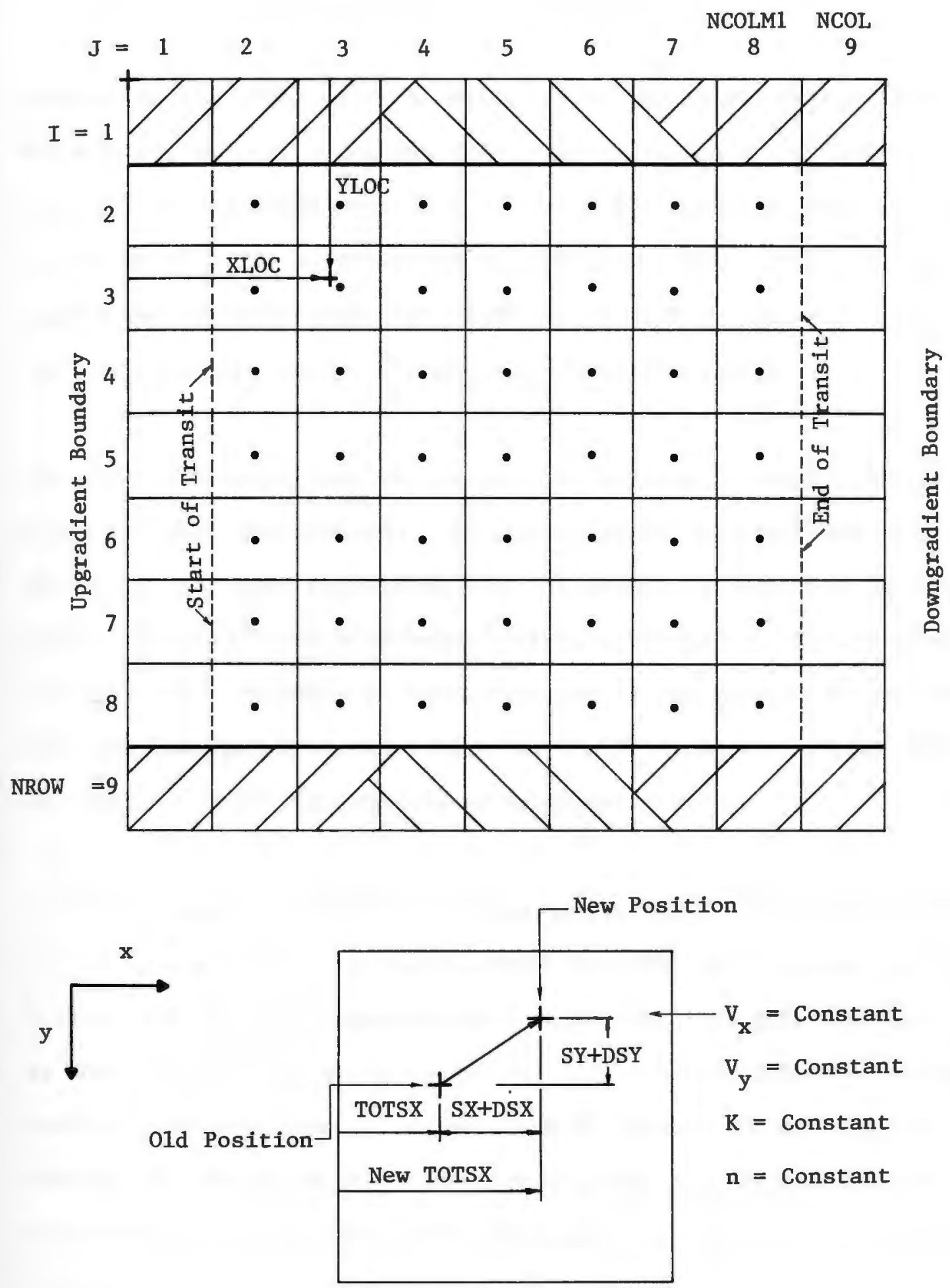

Figure B.7 - General Flow Region 
ments are calculated and added to those previously summed.

Eventually the water particle moves out of the block, either laterally, vertically, or both simultaneously. The code checks for lateral movement out of the block initially. If this has occurred, the code checks to verify that the movement was only into the next block. If movement was beyond the next block, this fact is recorded and printed out so that the time interval can be reduced in a later simulation.

The code then calculates the portion of the time interval spent in each block for this time interval. An adjustment of the displacements in the new block is made to account for the change in velocity in the new block. This adjusted displacement is recorded and a new time interval is begun. If the particle reached the end of the region, the portion of the last time interval remaining after the particle reached the boundary is subtracted from the computed total travel time.

A check is made to determine if the particle is displaced vertically out of the block as well. If not, then the next time interval is begun. However, if vertical movement has occurred into the next block, a check is made for block skipping as before. If vertical movement results in contact with a boundary, the particle is reflected back into the flow region. No additional adjustment in displacements is made in the event of horizontal and vertical block changes, a new time interval is simply begun.

In the event that the particle was displaced vertically out of the block 
but not horizontally out of the block, checks are made to record any skipping of blocks and reflection by the boundaries. An adjustment for displacements due to movement into the next block is made similar to the technique for lateral movement and a new time cycle begun.

Upon reaching the downgradient boundary, the following results are recorded in a array, tabularized and recorded on a data set file. These are then available for statistical manipulations and checks.

\author{
- Particle Number \\ - Simulation Number \\ - Starting $Y$ Coordinate \\ - $X$ and $Y$ Coordinate at the time after starting estimated for \\ travel to the region midpoint. \\ - Y Coordinate at Completion \\ - Time to Travel 50, 60, 70, 80 and $90 \%$ of the Region \\ - Total Travel Time
}

\title{
B.7 Procedures for the Analysis of Results
}

Monte Carlo simulations necessarily result in a great deal of output. It was necessary to develop a data collection and analysis system to properly deal with this. Data from this study is stored in disk data files as it's produced and saved for use and analysis later. Use has been made of a sophisticated data analysis system called the Statistical Analysis System (SAS) to assist in the analysis of the data from this study. 
A listing of the computer code referred to as STKBLKNP, which was adapted from that developed by Reiter (1981) is included in Appendix D. The code may be subdivided into 11 steps as listed below with the line numbers referring to the listing.

1. Data input and echo check of data on region size and parameters (lines 100 to 2000 ).

2. Selection of hydraulic conductivity form, autocorrelation development and stochastic generation of values for each Monte Carlo Simulation (lines 2010 to 3330 ).

3. Calculate actual statistical parameters of the hydraulic conductivity values generated (lines 3340 to 6280 ).

4. Computation of harmonic means of hydraulic conductivity at adjacent nodes for use in finite difference solution (lines $6290-6910)$

5. Finite difference solution for steady state heads (lines 6920 to 9170 ).

6. Compute flow quantity, equivalent hydraulic conductivity and write out results (lines 9180 to 11650 ).

7. Compute stream function and write out (lines 11660 to 13630 ). 
8. Compute velocities in all sections of the finite difference grid (lines 13640 to 14220 ).

9. Read in required data on dispersion and initialize parameters (lines 14230 to 14630$)$.

10. Water particles traverse the region (lines 14640 to 17670 ).

11. Results written out and stored on files (lines 17680 to 19580).

12. Subroutines to calculate means and correlations (lines 19590 to 20860 ).

The data stored in disk files is varied but consists of information on the hydraulic conductivity means and correlation for each simulation, travel times and dispersion, hydraulic head values both mean values and those for selected rows in each simulation. These data are then available for input to the SAS programs. SAS programs have been written to perform all types of statistical calculations and checks, as well as to plot these results.

\section{B.8 Discussion of the Results}

\section{B.8.1 Computer Time}

A considerable amount of computer simulation was performed during this 
study. One major limitation immediately encountered was the large amount of computer time required to invert the $(I-W)$ matrix. The size of the matrix increases very rapidly with the region size since a new row and column is added for each new finite difference block in the region.

Figure B.8 presents a plot of the required computer time for matrix inversion versus the number of nodes in the region. The three sizes computed were 49 ( $7 \times 7$ region), 144 (12 x 12 region) and 264 (12 × 22 region). These results suggest a non-linear relationship which is linearized by plotting on a semilog scale (log time) as presented in Figure B.8. This plot suggests a relationship defined by the following expression:

$$
\text { ln } \mathrm{T}=0.01075 \mathrm{~B}+0.23
$$

where $T$ is the time to complete the inverse and $B$ is the number of nodes in the mesh. The practical limit on region size based on this time requirement will depend on the computer system available; however, the largest region considered in this study was a $12 \times 22$ region.

A check of the accuracy of the inversion was performed by rewriting the inverse code to generate the Weighting matrix, retrieve the completed inverse matrix, FILTR, and pre-multiply the (I-W) matrix by the FILTR matrix. For the region considered, these results indicated that the inverse was accurate to five significant figures which is considered adequate. 


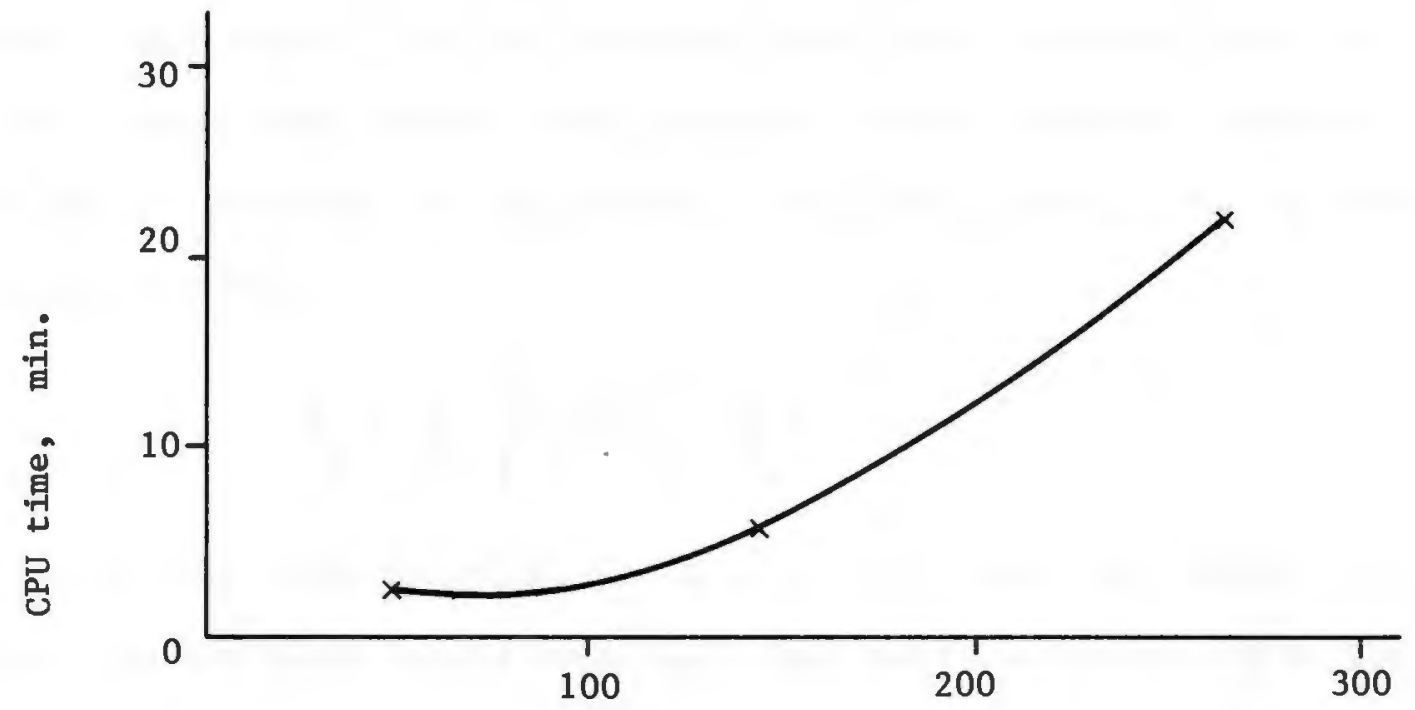

Number of Nodes

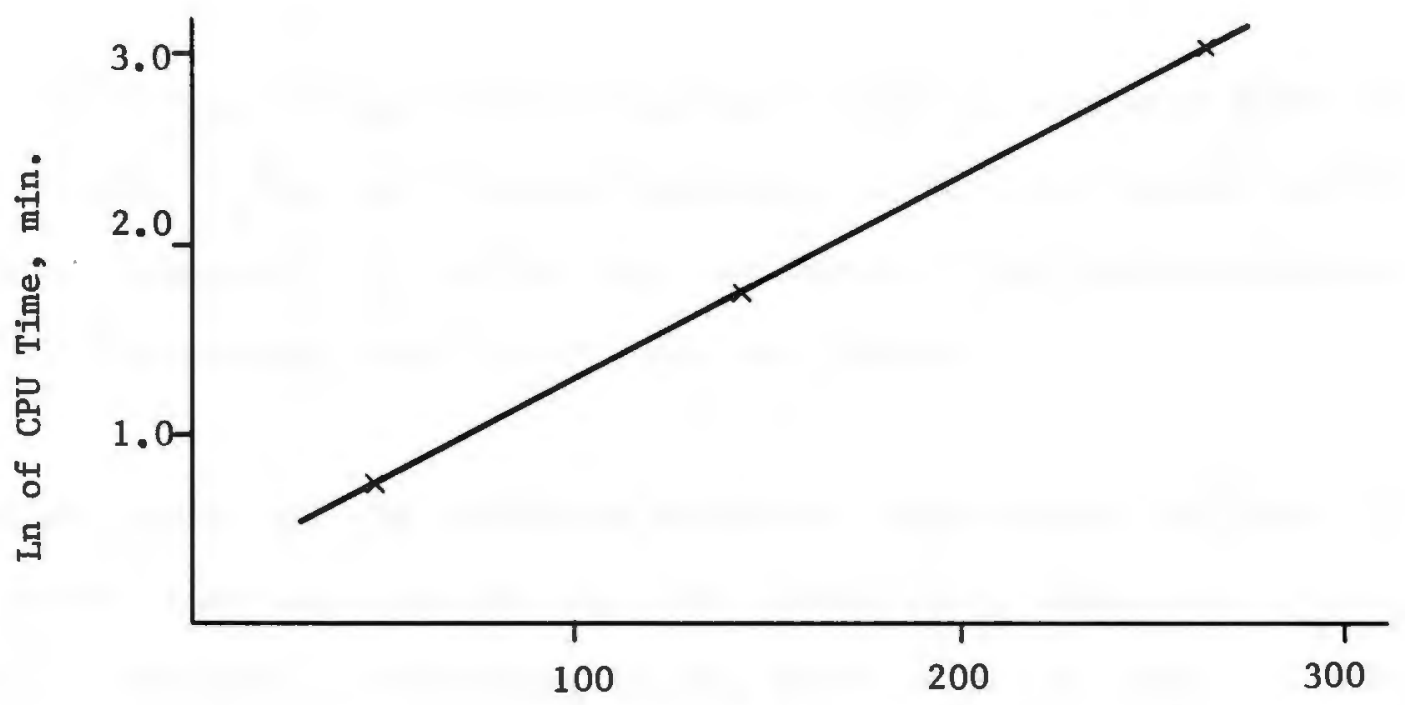

Number of Nodes

Figure B.8 - Computer Time Versus Number of Nodes 


\section{B.8.2 Effective Hydraulic Conductivity}

The arithmetic, geometric and harmonic means of all the simulations of hydraulic conductivity for the numerous Monte Carlo solutions were calculated. These mean values were compared to the effective hydraulic conductivity calculated on the basis of the flow quantity, $\mathrm{K}_{\mathrm{q}}$, by the following equation:

$$
\mathbf{K}_{\mathbf{q}}=\frac{\mathrm{Q}}{\mathrm{h}_{\mathrm{L}}}\left[\frac{\mathrm{L} \cdot \dot{S}}{\mathrm{H}}\right]=\frac{\mathrm{Q} \underline{\mathbf{s}}}{\mathrm{h}_{\mathrm{L}}}
$$

where $Q$ is the flow quantity, $h_{L}$ is the total head loss within the region, $L$ is the total length over which that head loss occurs, $H$ is the height of the flow region, and $S$ is a shape factor computed in accordance with a technique suggested by Warren and Price (1961) which will be presented later.

The value of $S$ is a shape factor which may be directly compared with the shape factor, $N_{D} / N_{F}$, in a flow net solution or the form factor in the method of fragments. $N_{D}$ and $N_{F}$, are the number of equipotential drops and flow lines respectively in the flow net solution.

A second measure of the effective hydraulic conductivity in terms of mean travel time was computed for each Monte Carlo simulation. This effective hydraulic conductivity on the basis of travel time, $K_{t}$, was calculated by making assumptions similar to those used in calculating $\mathrm{K}_{\mathrm{q}}$. Figure B.9 presents a typical layout of a flow region with approximately horizontal-uniform flow from left to right. The flow is 


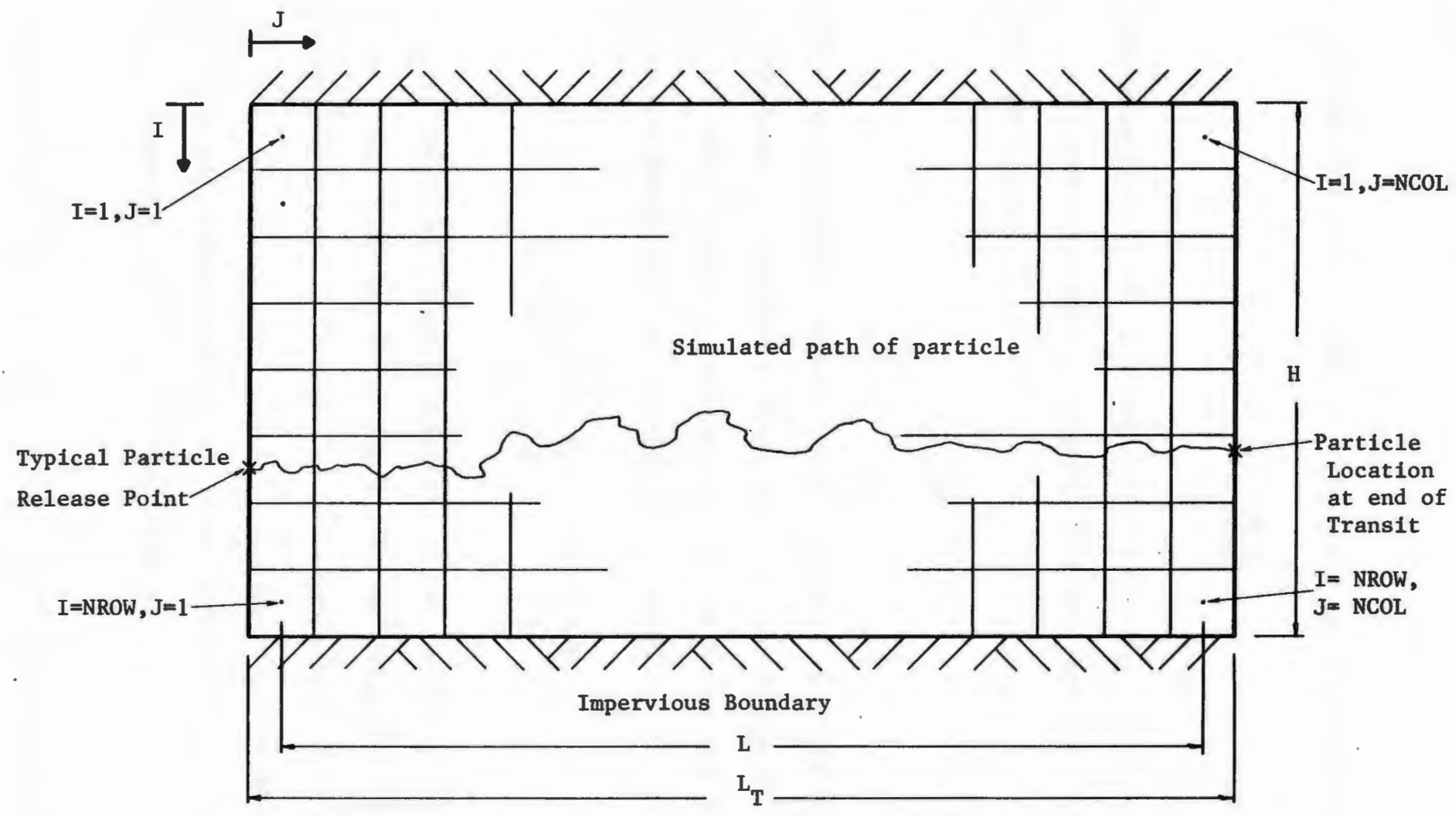

Along left boundary where $\mathrm{J}=1$, hydraulic head is maintained constant at value of $\mathrm{H}$ Along right boundary where $\mathrm{J}=\mathrm{NCOL}$, hydraulic head is maintained constant at value of $\mathrm{H}_{L}$ Mean head loss in flow region $=h_{L}=H_{u}-H_{L}$ 
not precisely uniform due to the dispersion present in the flow simulation.

The velocity of flow, $v$, may be calculated by the following formula:

$$
v=\frac{K i}{n}=\frac{K h_{L}}{n L}
$$

The mean velocity of all flow particles traversing the flow region, $\bar{v}$, may be calculated by dividing the length of the particle travel, $I_{T}$, by the mean travel time, $\bar{E}$, as below:

$$
\vec{v}=L_{T} / \bar{t}
$$

The effective hydraulic conductivity on the basis of travel time, $K_{t}$ has been calculated by setting $v$ equal to $\bar{v}$ in the above two equations, introducing the height of the region, $H$, by inserting in both the numerator and denominator and solving for $K_{t}$ resulting in the following:

$$
K_{t}=\frac{L_{T} \cdot n \cdot H}{t \cdot h_{L}} \cdot \frac{S_{t}^{\prime} I}{H}=\frac{L_{t} n}{\bar{t} h_{L}} \cdot S_{t}
$$

where $L_{T}$ is the total length of particle travel, $n$ is the porosity, $\bar{t}$ is the mean travel time and $L, H$ and $h_{L}$ are as defined above. $S_{t}$ is a shape factor computed in the same general manner as $\dot{S}$ above except that mean travel time rather than flow rate is used. $s_{t}$ is a modified shape factor which may be compared to the shape factors from a flow net, the method of fragments or to S calculated above.

Over thirty variations of flow region size and characteristics were 
studied to determine the relationship between these calculated means. Generally for each flow region, 40 Monte Carlo characterizations were done. The means and effective hydraulic conductivities for each of these was computed.

In addition to calculation of mean values for each of the Monte Carlo characterizations, a mean value of all Monte Carlo characterizations was computed. Correlation of the geometric mean and the two effective hydraulic conductivity values (flow quantity and travel time) were visually observed by plotting and also by computation of correlation coefficients. A typical plot of these results is presented in Figure B. 10 and the results are summarized in Table Bl.

The results of these computations support the observation that the geometric mean is the best measure of the effective hydraulic conductivity both in terms of flow quantity and travel time. The effective hydraulic conductivity calculated both by flow quantity, $K_{q}$ and travel time, $K_{t}$, are relatively close with the flow quantity value generally somewhat larger.

Two boundary flow configurations were considered in addition to uniform flow, i.e., flow from the left boundary to the right boundary of a rectangular region. The two flow configurations are presented in Figure B.11. The first flow configuration is described as $S$ flow for the case of flow from the upper three nodes of the left boundary to the lower three nodes of the right boundary. This case could simulate a general two dimensional flow case such as around an obstruction or low hydraulic 

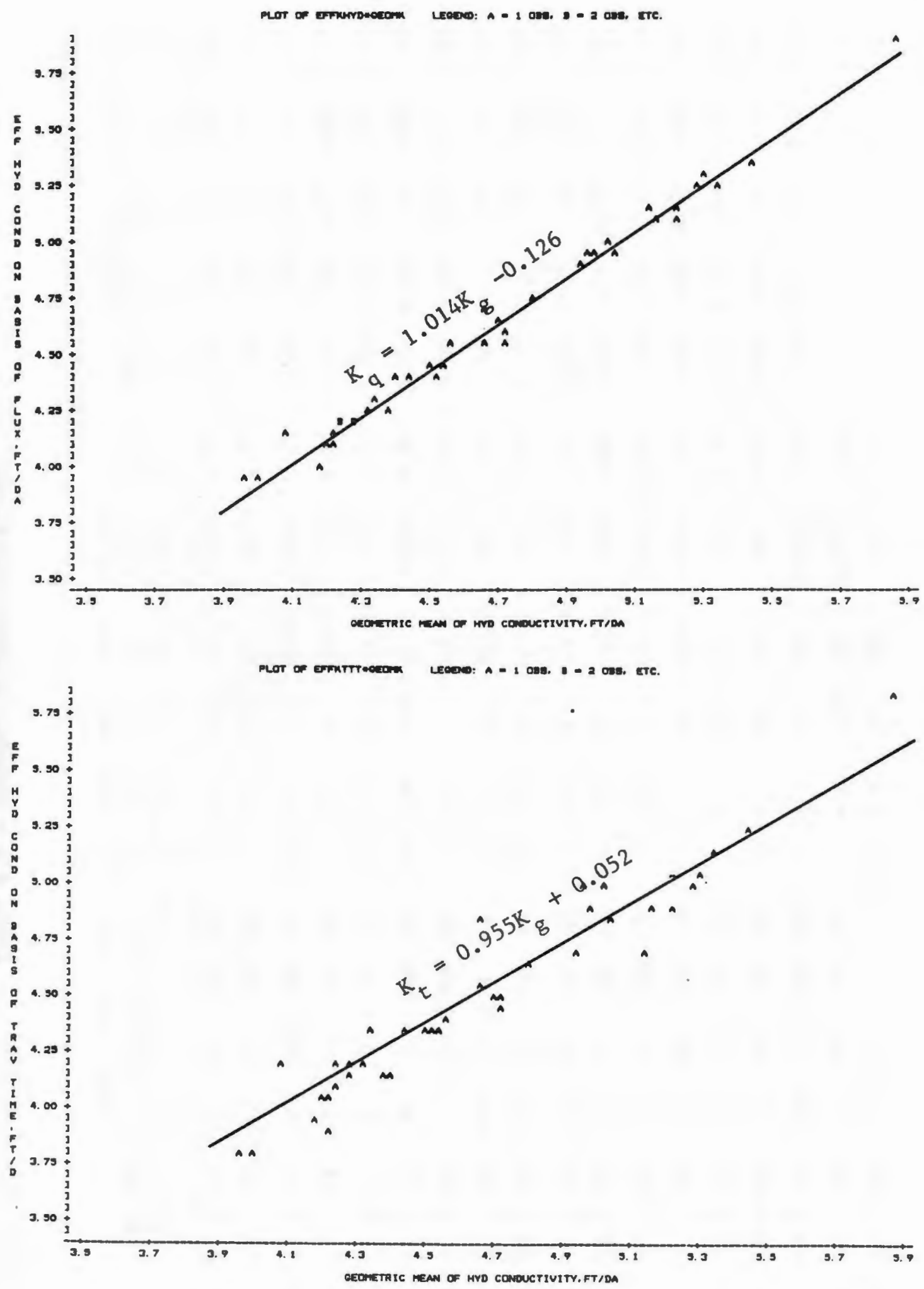

Figure B.10 - Correlation of Effective Hydraulic Conductivities with Geometric Mean of Hydraulic Conductivity 
TABLE B.1 - SUMMARY OF FLOW SIMULATIONS

\begin{tabular}{|c|c|c|c|c|c|c|c|c|c|c|c|c|c|c|c|c|c|}
\hline \multirow[b]{3}{*}{ File } & \multirow{3}{*}{$\begin{array}{c}\text { Region } \\
\text { Size }\end{array}$} & \multirow{3}{*}{$\begin{array}{l}\text { Block } \\
\text { Size }\end{array}$} & \multirow{2}{*}{\multicolumn{3}{|c|}{$\begin{array}{l}\text { Auto. Corr. } \\
\text { Data }\end{array}$}} & \multirow{3}{*}{$\begin{array}{l}\text { No. } \\
\text { of } \\
\text { Sims }\end{array}$} & \multirow{3}{*}{$\begin{array}{l}\text { No. } \\
\text { of } \\
\text { Wa- } \\
\text { ves }\end{array}$} & \multirow{3}{*}{$\begin{array}{l}\text { Time } \\
\text { Int., } \\
\text { Days }\end{array}$} & \multirow{3}{*}{$\begin{array}{l}\text { Kol- } \\
\text { mog } \\
\text { D } \\
\text { Stat }\end{array}$} & \multirow{3}{*}{$\begin{array}{l}Y \\
\text { Coor. } \\
\text { at } \\
\text { End }\end{array}$} & \multirow{3}{*}{$\begin{array}{l}\text { Total } \\
\text { Travel } \\
\text { Time, } \\
\text { Days }\end{array}$} & \multicolumn{6}{|c|}{ Means of Hydraulic Conductivity } \\
\hline & & & & & & & & & & & & \multirow{2}{*}{$\begin{array}{c}\overline{\text { Arith }} \\
\mathbf{K}_{\mathbf{a}}\end{array}$} & \multirow{2}{*}{$\begin{array}{c}\text { Geom } \\
\mathrm{K}_{\mathrm{g}}\end{array}$} & \multirow{2}{*}{$\underset{\mathrm{K}_{\mathrm{h}}}{\mathrm{Harm}}$} & \multirow{2}{*}{$\underset{\mathrm{q}}{\mathrm{Flux}}$} & \multirow{2}{*}{ T. Tim. } & \multirow{2}{*}{$\begin{array}{c}\text { S.Dev } \\
\text { K }\end{array}$} \\
\hline & & & $\mathbf{x}$ & $\mathbf{y}$ & & & & & & & & & & & & & \\
\hline 1 & $10 \times 20$ & $10 \times 10$ & .8 & .8 & .68 & 40 & 1 & 1.6 & .032 & 60.6 & 11200 & 5.02 & 4.63 & 4.28 & 4.5 & 4.44 & .10 \\
\hline 2 & $10 \times 20$ & $10 \times 20$ & .8 & .4 & .68 & 40 & 1 & 1.6 & .115 & 60.4 & 168.5 & 4.84 & 4.62 & 4.40 & 4.59 & 4.51 & 1.54 \\
\hline 3 & $10 \times 20$ & $10 \times 20$ & $.8 \mathrm{~L}$ & $.8 \mathrm{~L}$ & .68 & 40 & 1 & 1.6 & .15 & 59.5 & 171.1 & 5.01 & 4.65 & 4.32 & 4.57 & 4.44 & 2.01 \\
\hline 4 & $10 \times 10$ & $10 \times 10$ & .8 & .8 & .68 & 40 & 1 & 0.8 & .01 & 59.7 & 39.8 & 5.13 & 4.77 & 4.45 & 4.75 & 4.61 & 1.99 \\
\hline 5 & $5 \times 5$ & $5 \times 5$ & .8 & .8 & .68 & 40 & 2 & 6.4 & .01 & 35.0 & 9.5 & 5.13 & 4.78 & 4.46 & 4.80 & 5.28 & 1.90 \\
\hline 6 & $10 \times 20$ & $10 \times 20$ & .8 & .8 & .68 & 10 & 4 & 1.6 & .024 & 58.4 & 176.6 & 4.86 & 4.51 & 4.19 & .42 & 4.30 & 1.95 \\
\hline 7 & $10 \times 20$ & $10 \times 20$ & .8 & .8 & .68 & 44 & 3 & 1.6 & - & 58.6 & 234.3 & 5.05 & 4.69 & 4.36 & 4.63 & 4.54 & 1.99 \\
\hline 8 & $10 \times 20$ & $10 \times 20$ & .8 & .4 & 1.0 & 40 & 1 & 1.6 & .01 & 60.0 & 173.0 & 5.09 & 4.60 & 4.15 & 4.54 & 4.39 & 2.40 \\
\hline 9 & $10 \times 20$ & $10 \times 20$ & .8 & .8 & .68 & 40 & 1 & 1.6 & .01 & 59.9 & 169.7 & 5.04 & 4.68 & 4.34 & 4.62 & 4.52 & 2.02 \\
\hline 10 & $10 \times 40$ & $10 \times 20$ & .8 & .8 & .68 & 40 & 1 & 3.2 & .01 & 59.6 & 703.4 & 4.99 & 4.62 & 4.28 & 4.53 & 4.45 & 2.02 \\
\hline 11 & $10 \times 40$ & $10 \times 20$ & .8 & .8 & .68 & 40 & 1 & 0.3 & .15 & ( & 6 & 5.06 & 4.68 & 4.32 & 4.59 & 4.51 & 2.08 \\
\hline 12 & $10 \times 40$ & $10 \times 20$ & .8 & .8 & .68 & 40 & 1 & 6.4 & .014 & 59.2 & 702.2 & 5.02 & 4.64 & 4.29 & 4.54 & 4.46 & 2.08 \\
\hline 13 & $10 \times 40$ & $10 \times 10$ & .8 & .8 & .68 & 4 & 1 & 3 & .04 & . & 697.0 & 5.02 & 4.64 & 4.29 & 4.55 & 4.48 & 2.08 \\
\hline 14 & $20 \times 40$ & $10 \times 10$ & .8 & .8 & .68 & 20 & 1 & 3.2 & .15 & 111.7 & 700.0 & 4.99 & 4.61 & 4.27 & 4.54 & 4.46 & 2.09 \\
\hline 15 & $20 \times 40$ & $10 \times 20$ & .8 & .8 & .68 & 20 & 1 & 3.2 & .15 & 111.2 & 03 & 4.30 & 4.62 & 4.28 & 4.55 & 4.46 & .03 \\
\hline 16 & $10 \times 60$ & $10 \times 20$ & .8 & .8 & .68 & 40 & 1 & 4.8 & .15 & 59.5 & 1576.2 & 5.06 & 4.67 & 4.31 & 4.57 & 4.49 & 2.11 \\
\hline 17 & $10 \times 60$ & $10 \times 10$ & .8 & .8 & .68 & 40 & 1 & 4.8 & .052 & 59.5 & 1576.7 & 5.06 & - & - & - & - & 2.14 \\
\hline 18 & $10 \times 20$ & $10 \times 20$ & 0 & .0 & - & 1 & 40 & .6 & .15 & 60.2 & 151.9 & 5.0 & 5.0 & 5.0 & 5.0 & 5.0 & 0.0 \\
\hline
\end{tabular}


TABLE B.1 - SUMMARY OF FLOW SIMULATIONS (CONTINUED)

\begin{tabular}{|c|c|c|c|c|c|c|c|c|c|c|c|c|c|c|c|c|c|}
\hline \multirow[b]{3}{*}{ File } & \multirow{3}{*}{$\begin{array}{l}\text { Region } \\
\text { Size }\end{array}$} & \multirow{3}{*}{$\begin{array}{l}\text { Block } \\
\text { Size }\end{array}$} & \multirow{2}{*}{\multicolumn{3}{|c|}{$\begin{array}{l}\text { Auto. Corr. } \\
\text { Data }\end{array}$}} & \multirow{3}{*}{$\begin{array}{l}\text { No. } \\
\text { of } \\
\text { Sims }\end{array}$} & \multirow{3}{*}{$\begin{array}{l}\text { No. } \\
\text { of } \\
\text { Wa- } \\
\text { ves }\end{array}$} & \multirow{3}{*}{$\begin{array}{l}\text { Time } \\
\text { Int., } \\
\text { Days }\end{array}$} & \multirow{3}{*}{$\begin{array}{l}\text { Kol- } \\
\text { mog } \\
\text { D } \\
\text { Stat }\end{array}$} & \multirow{3}{*}{$\begin{array}{l}Y \\
\text { Coor. } \\
\text { at } \\
\text { End }\end{array}$} & \multirow{3}{*}{$\begin{array}{l}\text { Total } \\
\text { Travel } \\
\text { Time, } \\
\text { Days }\end{array}$} & \multicolumn{6}{|c|}{$\begin{array}{r}\text { Heans of Hydraulic } \\
\mathrm{ft} / \mathrm{day}\end{array}$} \\
\hline & & & & & & & & & & & & Arith & & Harm & Flux & & S.Dev \\
\hline & & & $\mathbf{x}$ & y & & & & & & & & & & 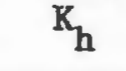 & & & K \\
\hline 12 & $10 \times 20$ & $10 \times 20$ & .8 & .2 & 1.0 & (7) & 1 & & & 60.7 & & 5.11 & 4.65 & 4.23 & 4.62 & 4.47 & 2.35 \\
\hline 20 & $10 \times 20$ & $10 \times 20$ & .8 & .2 & 0.9 & 40 & 1 & 1.6 & .15 & 60.2 & .8 & 5.005 & .64 & 4.3 & 4.6 & 4.7 & .04 \\
\hline & $10 \times 60$ & $10 \times 20$ & .0 & .8 & .68 & 40 & 1 & 1.0 & .048 & 60.3 & 314.5 & 4.96 & 4.58 & 4.23 & 4.48 & 4.41 & 2.05 \\
\hline-2 & $10 \times 60$ & $10 \times 20$ & 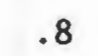 & .8 & .68 & 40 & 1 & .44 & .066 & 60.5 & 143 & 5.07 & 4.68 & 4.33 & 4.59 & 4.50 & 2.07 \\
\hline 23 & $10 \times 20$ & $10 \times 20$ & .8 & .8 & .68 & 40 & 1 & 1.6 & - & 98.4 & 228.0 & 5.12 & 4.72 & 4.36 & 4.62 & 4.50 & 2.12 \\
\hline- & $10 \times 20$ & $10 \times 20$ & 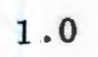 & 1.0 & 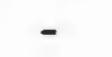 & $\mathbf{F}$ & 133 & 1.6 & - & . & 2100 & 5.0 & . & 5.0 & 5.0 & 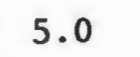 & 0.0 \\
\hline & $10 \times 20$ & $10 \times 20$ & 0 & 1.0 & - & 1 & 40 & 1.6 & - & 98.8 & 20 & 5.0 & 5. & 5.0 & 5.0 & 5.0 & 0.0 \\
\hline 26 & $10 \times 20$ & $10 \times 20$ & 0.0 & 0.4 & .84 & 40 & 1 & 1.6 & & 00. & 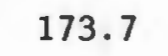 & 4.90 & 4.55 & 4.23 & 4.51 & 4.39 & 1.90 \\
\hline 27 & $10 \times 20$ & $0 \times 20$ & 8 & 8 & .6 & 40 & 1 & 6 & .01 & 58.8 & 254.91 & 5.01 & 3.54 & 2.51 & 3.32 & 3.06 & 4.786 \\
\hline 28 & $10 \times 20$ & $10 \times 20$ & .8 & .8 & .68 & 40 & 1 & 1.6 & & 60.0 & 153.11 & 5.01 & 4.98 & 4.96 & 4.98 & 4.97 & 0.50 \\
\hline 23. & $20 \times 40$ & $10 \times 20$ & .8 & .8 & .68 & 20 & 1 & 3.2 & & 108.0 & $\cdot 4$ & 2 & 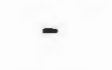 & - & - & 2 & .47 \\
\hline 30 & $10 \times 20$ & $10 \times 20$ & .4 & .3 & .95 & 40 & 1 & 1.6 & .15 & 59.8 & 170.5 & 5.01 & 4.66 & 4.34 & 4.58 & 4.47 & 1.97 \\
\hline
\end{tabular}

Note: Uniform flow in all files except 7 and 24 which simulate S Flow and 23 and 25 with cutoff.

Values listed for $K_{q}$ and $k_{t}$ have been adjusted by use of the shape factor $s$. 

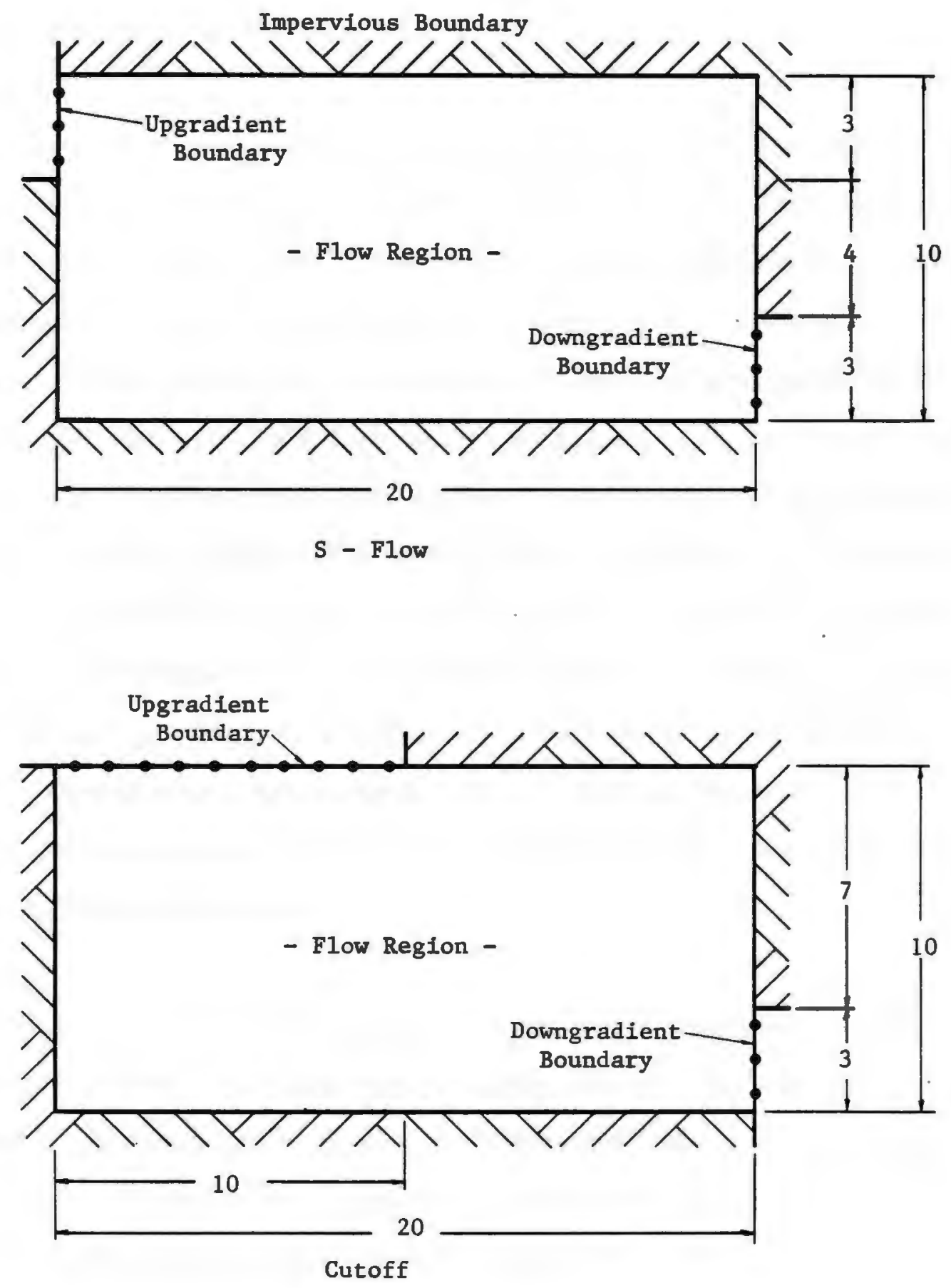

Figure B.11 - Alternate Flow Configurations 
conductivity occlusion. The second flow configuration is described as cutoff for the case of flow from the left upper boundary to the bottom right boundary. This case is intended to simulate flow beneath a dam by representing one half of the flow region.

Warren and Price (1961) have indicated that the geometric mean of the hydraulic conductivity is a good approximation of the effective hydraulic conductivity even for non-uniform flow provided a shape factor is utilized to adjust the value for the shape of the flow region. They suggest that the magnitude of this shape factor be determined by first calculating the effective hydraulic conductivity of the flow region using a singular known value of hydraulic conductivity. This effective value of hydraulic conductivity should then be divided into the singular known value and the result is the shape factor. The hydraulic conductivity value determined for the non-uniform flow case with the log normal distribution of hydraulic conductivity is multiplied by the shape factor before comparing with the geometric mean. This approach is similar to that technique embodied in a flow net solution or the one incorporated in the method of fragments.

The results of this study support the technique suggested by Warren \& Price (1961) for calculation of a shape factor. For the cases considered, the computed values of effective hydraulic conductivity generally fall between the geometric and harmonic means and is closest to the geometric mean as presented in Figure B12. 


\begin{tabular}{|c|c|c|c|c|c|c|c|c|c|c|c|c|c|c|c|}
\hline \multirow[b]{2}{*}{ File No. } & \multirow[b]{2}{*}{ Flow Type } & \multirow[b]{2}{*}{$\mathbf{K}$} & \multirow[b]{2}{*}{$\begin{array}{c}\text { Arith } \\
\mathrm{K}_{\mathrm{a}}\end{array}$} & \multicolumn{6}{|c|}{ Hydraulic Conductivity Means } & \multicolumn{5}{|c|}{ Shape Factors } & \multirow{2}{*}{$\begin{array}{c}\text { Method } \\
\text { of } \\
\text { Fragment } \\
\emptyset_{\mathrm{m}} \\
\end{array}$} \\
\hline & & & & $\begin{array}{c}\text { Geom } \\
\mathrm{K}_{8}\end{array}$ & $\begin{array}{c}\text { Ha rm } \\
\mathrm{K}_{\mathrm{h}}\end{array}$ & $\mathrm{K}_{\mathrm{q}}$ & $\mathrm{K}_{\mathrm{q}}$ & $K_{t}$ & $K_{t}$ & $\mathbf{S}$ & $\mathbf{S}$ & $s_{t}$ & $s_{t}$ & $\begin{array}{l}\text { Flow } \\
\text { Net } \\
\mathrm{N}_{\mathrm{d}} / \mathrm{N}_{\mathrm{f}}\end{array}$ & \\
\hline 9 & Uniform & 2 & 5.04 & 4.68 & 4.34 & 4.62 & 4.62 & 4.50 & 4.50 & 1.0 & 1.9 & 1.0 & 1.9 & 1.9 & 1.9 \\
\hline 7 & S-Flow & 2 & 5.05 & 4.69 & 4.36 & 3.17 & 4.63 & 3.26 & 4.54 & 1.46 & 2.78 & 1.39 & 2.66 & 3.0 & 2.91 \\
\hline 24 & & 0 & 5.0 & 5.0 & 5.0 & 3.42 & 5.0 & 3.59 & 5.0 & & & & & & \\
\hline 23 & Cutoff & 2 & 5.12 & 4.72 & 4.36 & 2.79 & 4.62 & 3.41 & 4.50 & 1.66 & 3.15 & 1.32 & 2.57 & 3.25 & - \\
\hline
\end{tabular}

Note: $K_{q}$ and $K_{t}$ are the equivalent mean values of $K_{q}$ and $K_{t}$ after adjusting by the shape factor. 


\section{B.8.3 Autocorrelation Coefficients and Integral Scale}

The stochastic nearest neighbor process model was utilized to generate random ensembles of hydraulic conductivity meshes which are autocorrelated. Each of the individual representations within the ensemble of representations making up each Monte Carlo simulation was analyzed to determine the degree of correlation by row and column. The formulation below utilized to calculate this value may be found in many references, e.g. Smith (1981).

$$
\operatorname{Rho}(L)=\frac{\frac{1}{n-L} \sum_{i=1}^{n-L}\left(K_{i}-\bar{K}\right)\left(K_{i+L}-\bar{K}\right)}{\frac{1}{n-1} \sum_{i=1}^{n}\left(K_{i}-\bar{K}\right)^{2}}
$$

In this equation Rho $(\mathrm{L})$ is the autocorrelation coefficient for lag $\mathrm{L}$. $\mathrm{L}$ is a whole number from zero to the number of elements in the row or column minus one. $K_{i}$ is the value of hydraulic conductivity at point $i$, and $K$ is the mean value. The denominator is the variance of the hydraulic conductivity. It may be easily shown that if $\mathrm{L}$ equals zero, then Rho (0) equals 1.0 .

A graph of a typical result of this computation for lags from one to three is presented in Figure B.13. The concept of integral scale as discussed by Smith and Freeze (1979) is utilized herein to conceptualize the degree of dependency of the hydraulic conductivity value at one node with the value at adjacent nodes. The integral scale has been estimated as the summation of the positive autocorrelation coefficients times the 


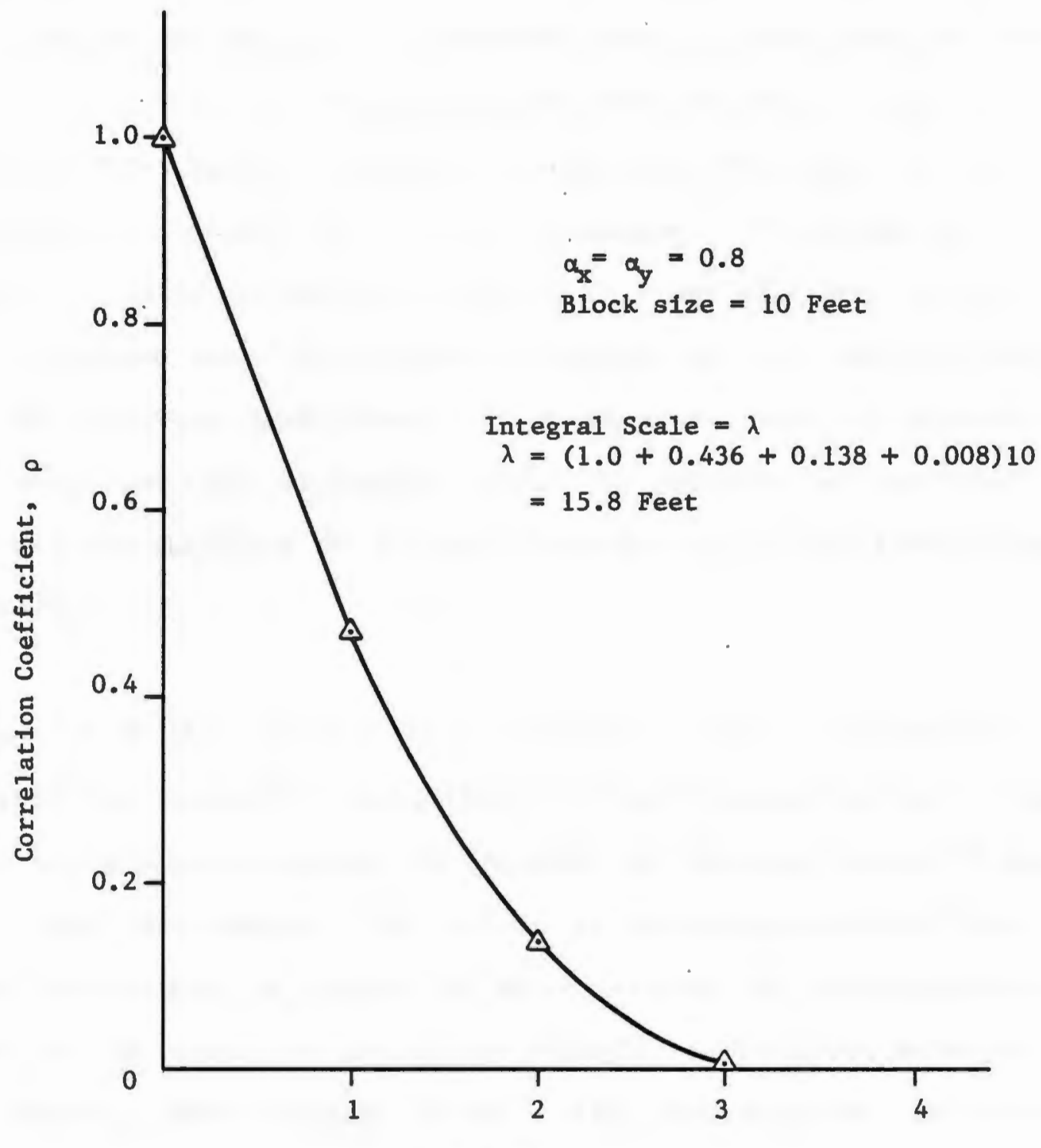

Lag Number

Figure B.13 - Correlation Coefficient Versus Lag 
block size. As indicated, the integral scale, $\lambda$, equals 15.8 for the data in Figure B.13.

During the course of this work, the nature of the governing equation for autocorrelation of hydraulic conductivity values at the boundaries was raised. An alternative methodology was written and a Monte Carlo simulation investigated. Figure B.14 presents the mean row autocorrelation coefficients by the two techniques. Utilizing the coefficients resulted in integral scales of 14.9 and 12.4 feet for the $x$ and $y$ directions using the original technique and 14.4 and 12.3 feet using the alternate formulation. It would appear that at least for these conditions the alternative technique provided no significant difference and therefore no further investigation of this alternative was conducted.

Irrespective of this finding, there was still concern over the autocorrelation of hydraulic conductivity in the boundary blocks. This problem was mitigated somewhat by deleting the boundary blocks of the region after development. The effects on the autocorrelation coefficients may be seen on Figure B.15 which presents the autocorrelation coefficients by rows of the $\log$ of the hydraulic conductivity before and after deleting these boundary blocks. Also presented are the autocorrelation coefficients by rows of this same simulation after the log transformation to hydraulic conductivity. The results are fairly typical of the simulations conducted and indicate a slight loss of correlation when the boundaries blocks were deleted and some additional loss when the log transformation was completed. This total loss was 


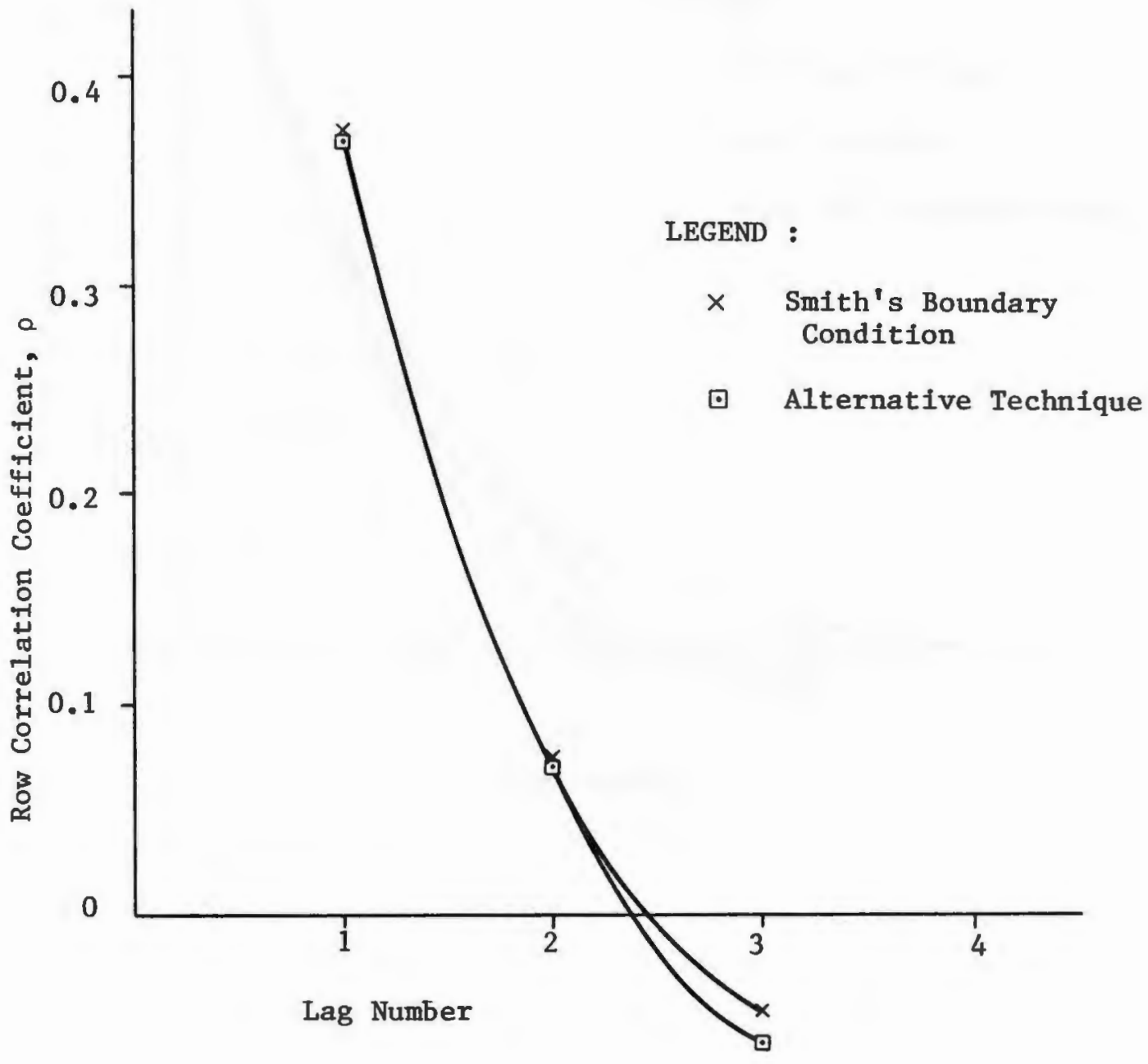

Figure B.14 - Comparison of Auto Correlation Coefficients 


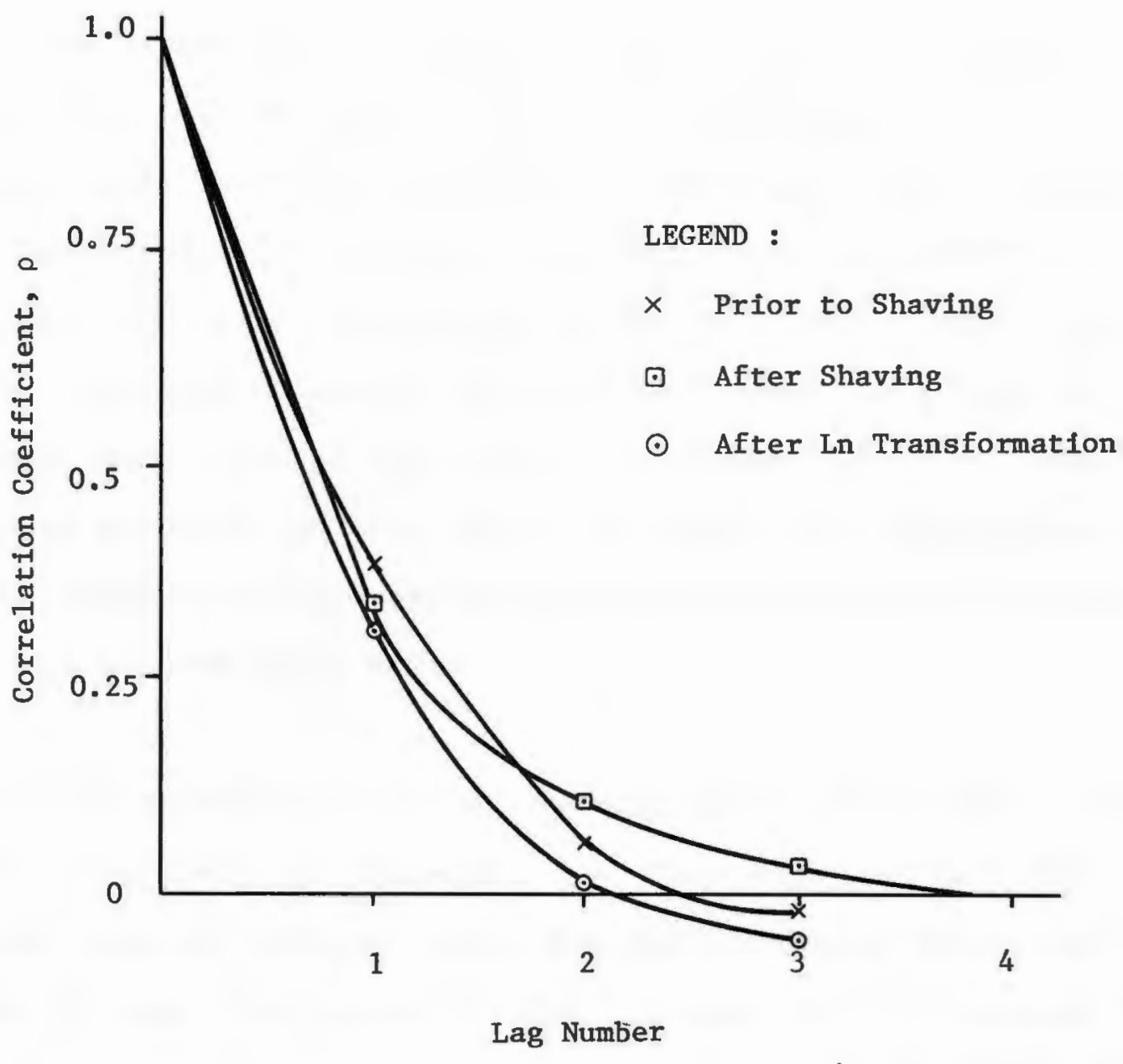

Figure B.15 - Effects of Shaving and Stacking of Blocks on Autocorrelation 
rather slight, however. The reduction in the integral scale is considerably less.

The effect of the region size on the integral scale is demonstrated in Figure B.16. It is clear that as the region size increases, the integral scale increases, although at a decreasing rate. This figure also demonstrates the effects of stacking blocks of internally autocorrelated hydraulic conductivity meshes to achieve larger regions without utilizing a larger FILTR matrix. There is a loss in the integral scale although this loss is relatively small even when the region is increased by three times. The figure also demonstrates that as the region size increases, the integral scale continues to increase, even with the same FILTR matrix.

Figure B.17 demonstrates that the integral scale changes with a change in the autocorrelation parameter, $\alpha$. This plot indicates that the integral scale in both the $x$ and $y$ direction increases with an increase in the $\alpha_{y}$ term. The increase in the y integral scale is expected, but the increase in the integral scale in the $x$ direction may not be. This increase in the integral scale in the $x$ direction resulting from the increase in the $a_{y}$ term likely results from a general increase in correlation of all terms in the mesh.

\section{B.8.4 Sensitivity of Ensemble Means and Variances to the Number of Simulations}

Computer computations are relatively inexpensive. However, the prac- 


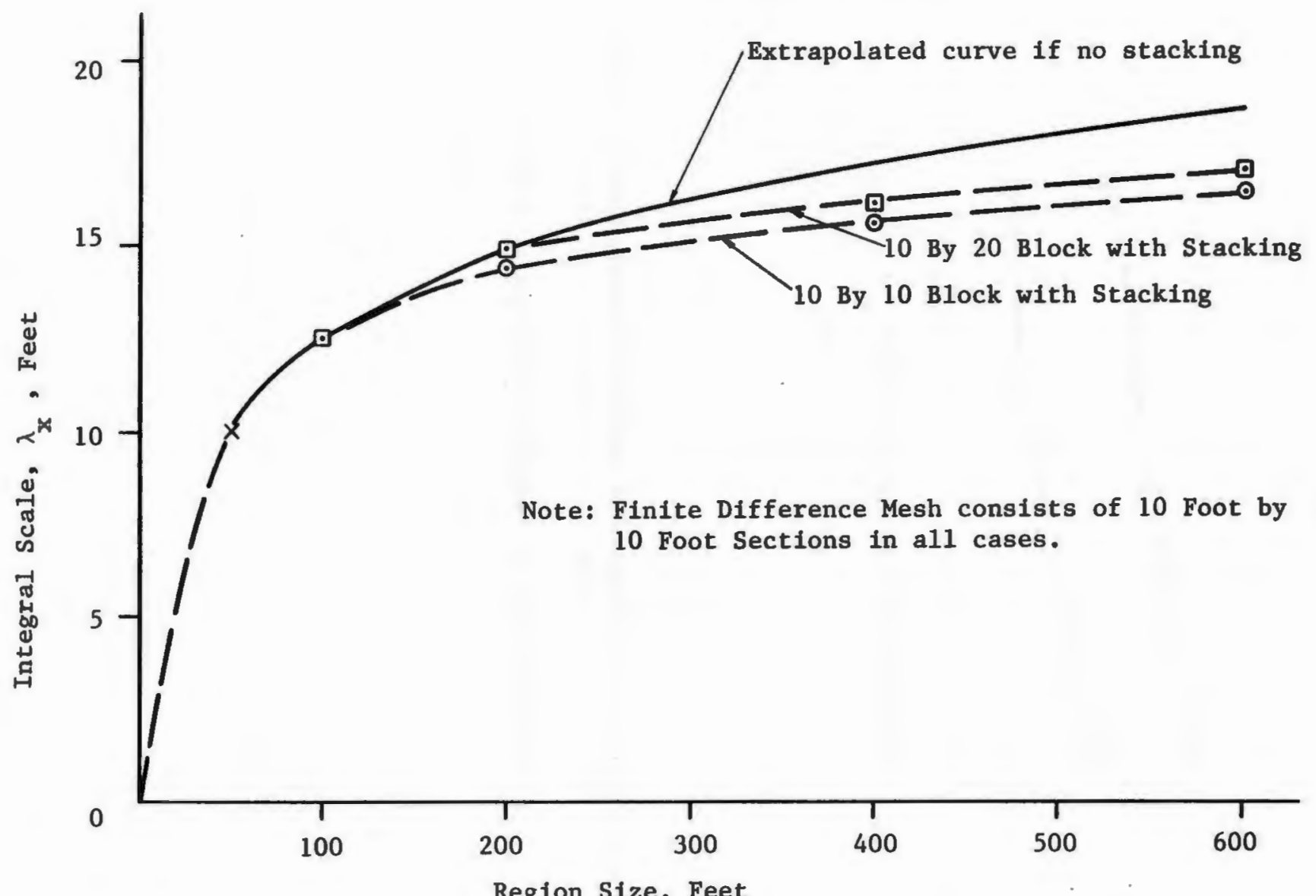

Figure B.16 - Effects of Region Size on Autocorrelation 


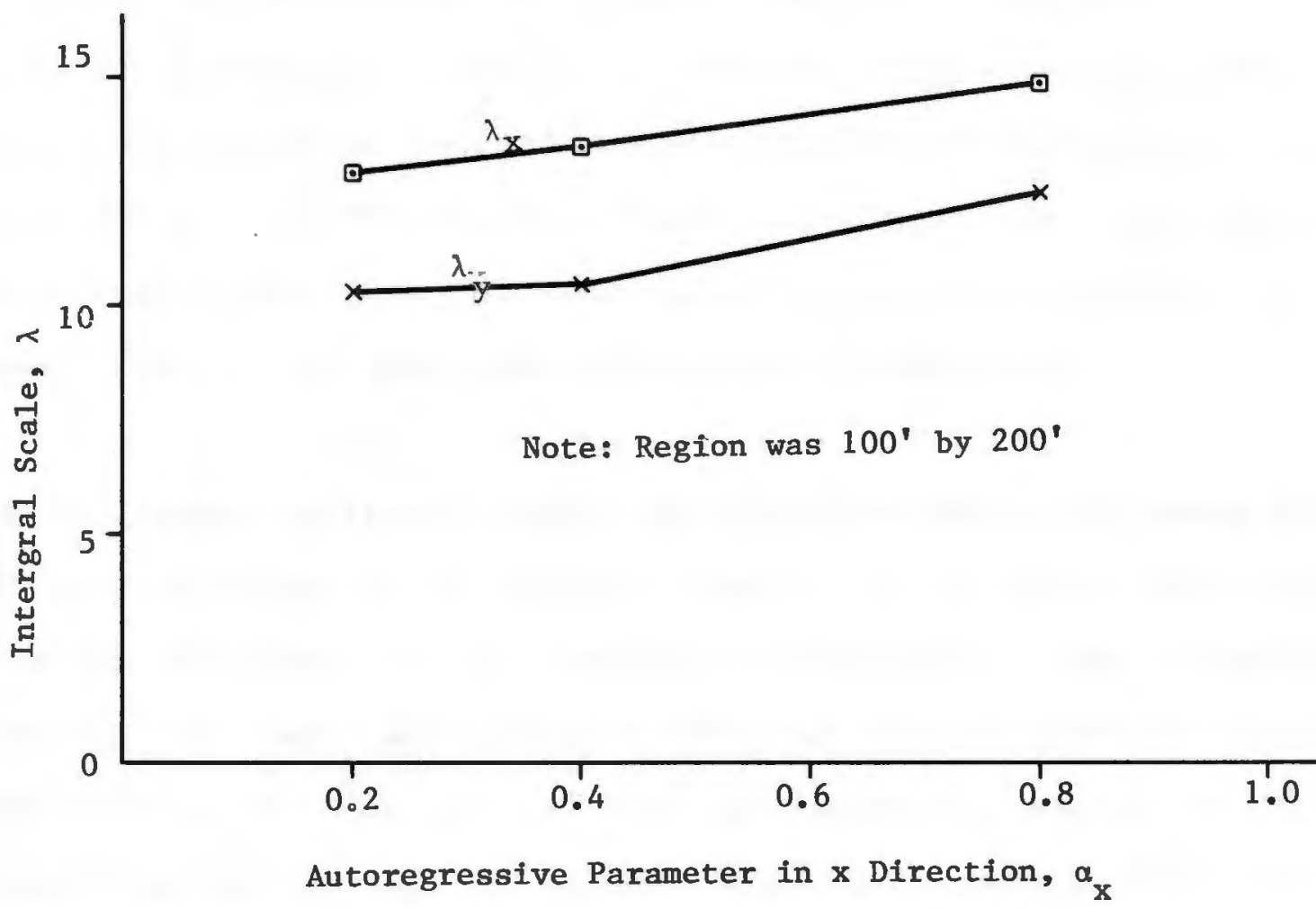

Figure B.17 - Effects of Autoregressive Parameters on Integral Scale 
tioner does not want to do more computations than are necessary to obtain reliable estimates. A study of the number of simulations required to compute reliable mean values of various parameters was then conducted.

Initially consider the mean and standard deviation of the hydraulic conductivity formulations. Figure B.18 presents a plot of an accumulating mean of the arithmetic mean and standard deviation of the hydraulic conductivity for one of the Monte Carlo simulations. This plot demonstrates that these cumulative mean values very rapidly approach a constant value, in this case after approximately 8 simulations.

The SAS program written to compute the cumulative mean of the means and standard deviation is an inexact estimate of the actual mean and standard deviation of all preceding observations. The procedure computes the mean and standard deviation of the previous three observations. A running mean value of these two results is then computed as each new mean and standard deviation is computed for the new observation and the two left from the earlier group. The results have been found accurate to approximately 3 to 4 decimal places for the mean value. However the mean of the running standard deviation computation by this technique may only be used as a qualitative measure of the trend and its stability. A true measure of the actual standard deviation can only be computed after the entire ensemble is sampled.

Figure B.19 and B.20 present similar plots for the effective hydraulic conductivity in terms of flow quantity, $\mathbf{K}_{q}$, and travel time, $K_{t}$, 

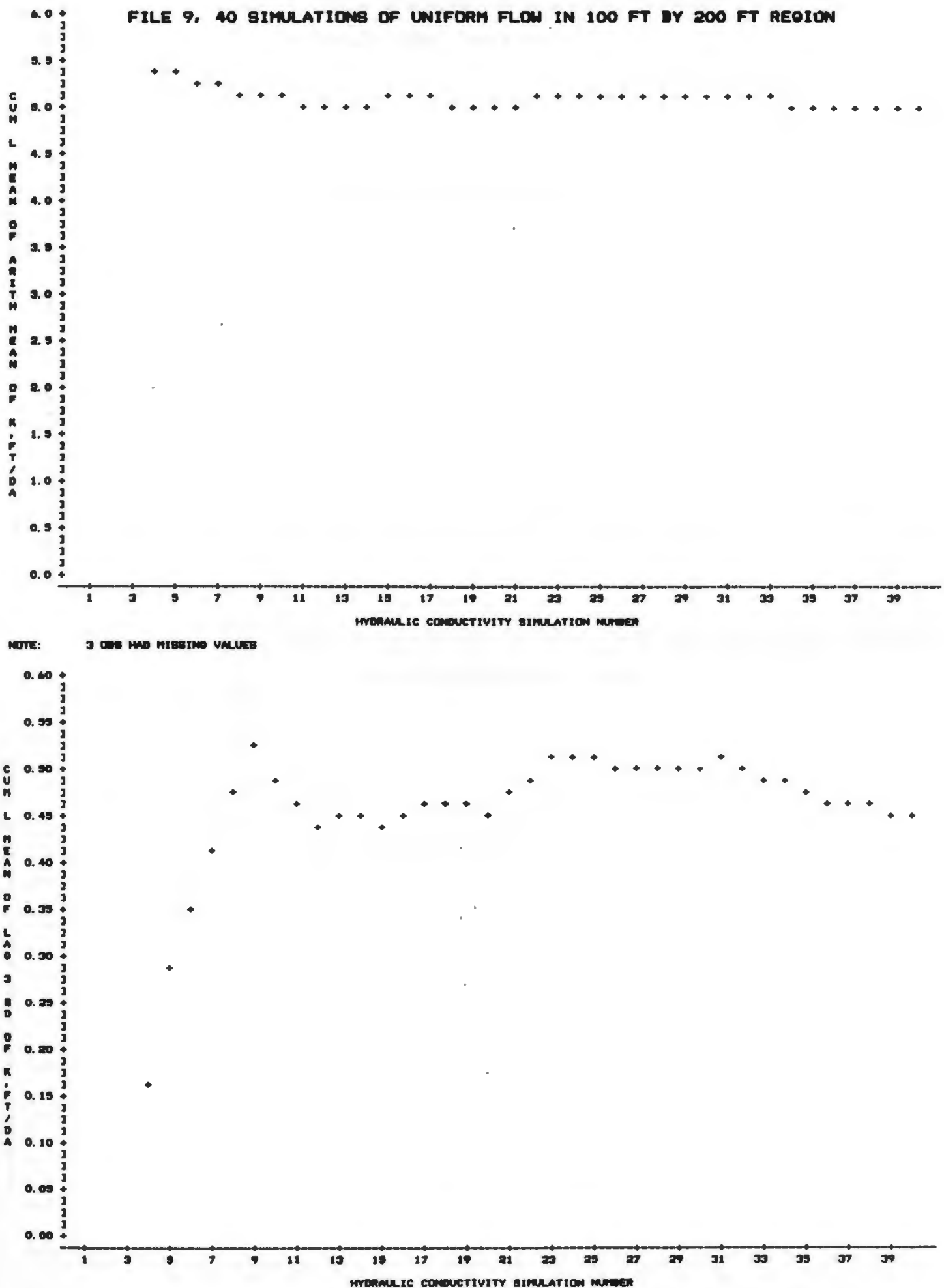

Figure B.18 - Cumulative Mean of the Arithmetic Mean and Lag 3 Standard Deviation of Hydraulic Conductivity Versus Simulation Number 
FILE 9, to gIMLATIOWB OF UNIFOW FLON IN $100 \mathrm{FT}$ IY $200 \mathrm{FT}$ REOION

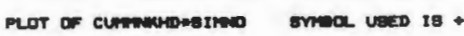
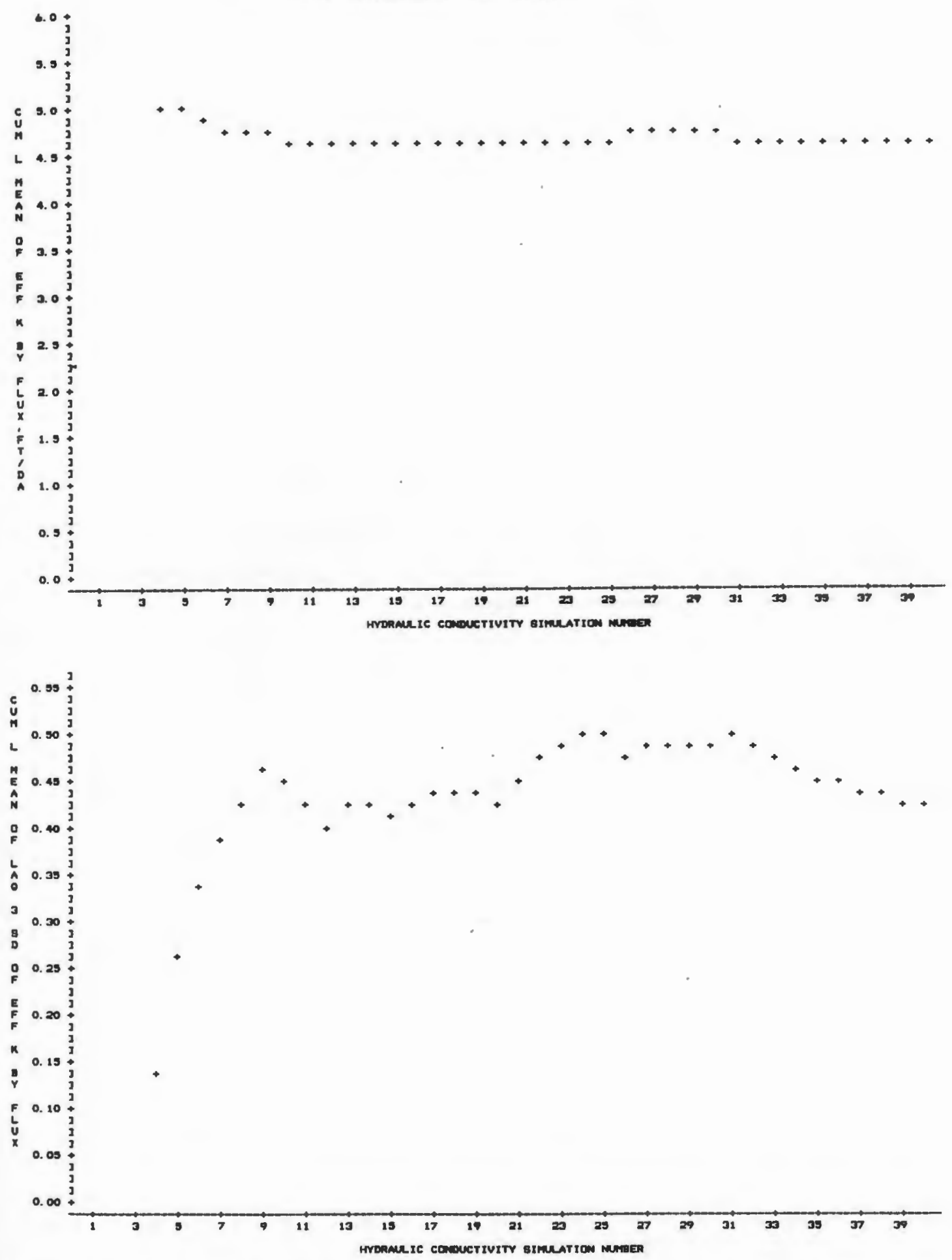

Figure B.19 - Cumulative Mean of the Mean and Lag 3 Standard Deviation of Effective Hydraulic Conductivity (Flux) Versus Simulation Number 
FIL 9.40 BImLATION OF UWIFDW FLON IN $100 \mathrm{FT}$ or $200 \mathrm{FT}$ AEOION

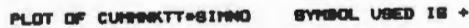
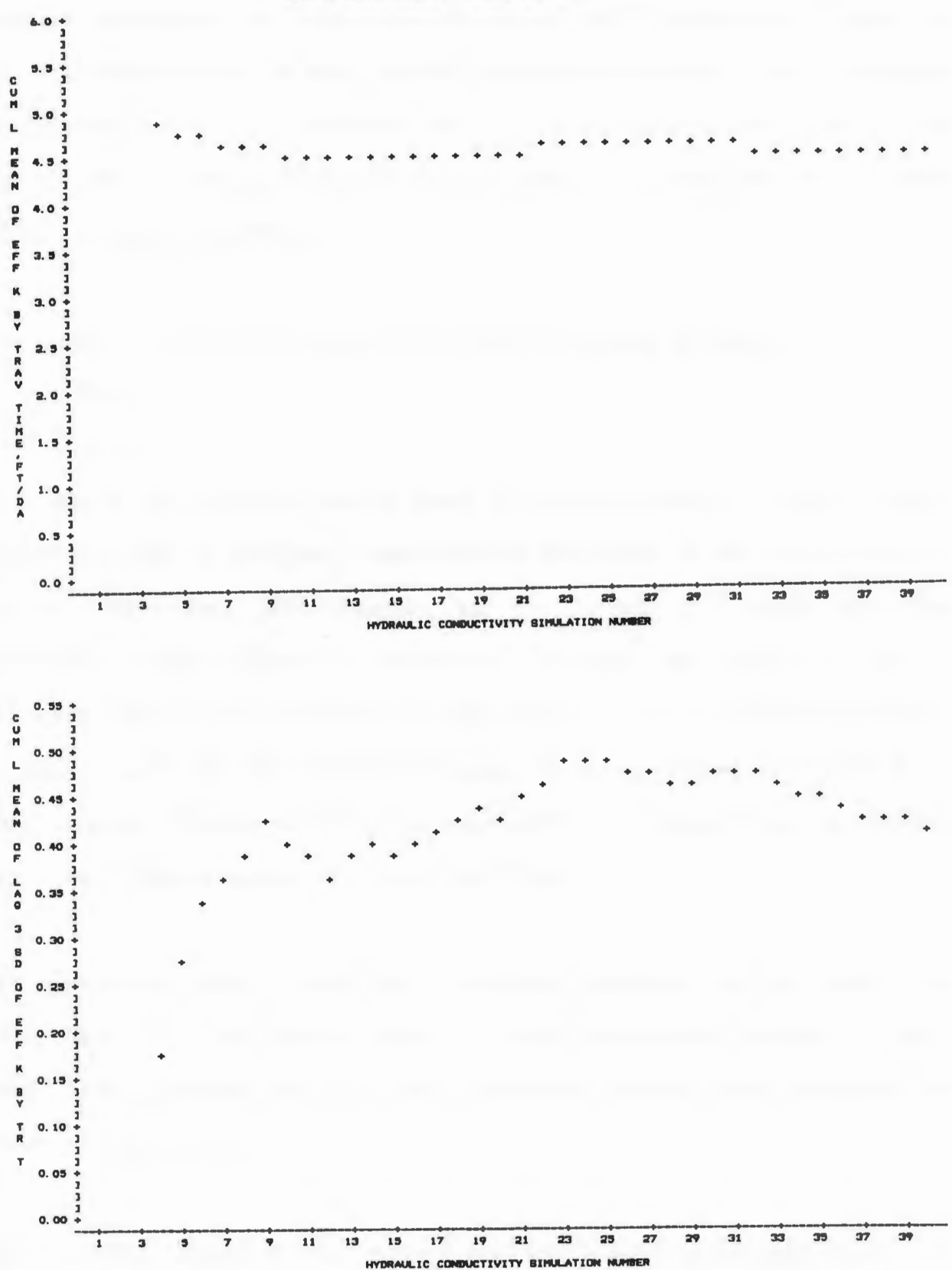

Figure B.20 - Cumulative Mean of the Mean and Lag 3 Standard Deviation of Effective Hydraulic Conductivity (Travel Time) Versus Simulation Number 
respectively. Both of these indicate that a fairly constant value is rapidly approached, in this case, in only 7 to 10 simulations. However, the cumulative means of the standard deviation for both terms fluctuates considerably. A truly constant value is not achieved even after the 40 simulations in the ensemble although after the first 20 , the fluctuations are greatly reduced.

\section{B.8.5 Sensitivity of Ensemble Means and Variances to Number of Particles}

The number of particles which need to be simulated to reach a fairly constant value of the mean and standard deviation of the travel time is another important consideration in the amount of computation time required. A SAS program was written to calculate the cumulative mean of the mean and standard deviation of the travel time for particle transit. A typical plot of the results for one run is presented in Figure B.21. This figure illustrates that a mean value of travel time is reached after only approximately 20 water particles.

The cumulative mean of the lag 3 standard deviation of the travel time fluctuates 20 or 40 percent after 20 water particles reaching a fairly consistent, although still slightly decreasing value after approximately 80 water particles.

Fried (1975) gives an expression for the longitudinal dispersion coefficient assuming that the development of particle concentration at some distant point is normally distributed. He illustrates that this 

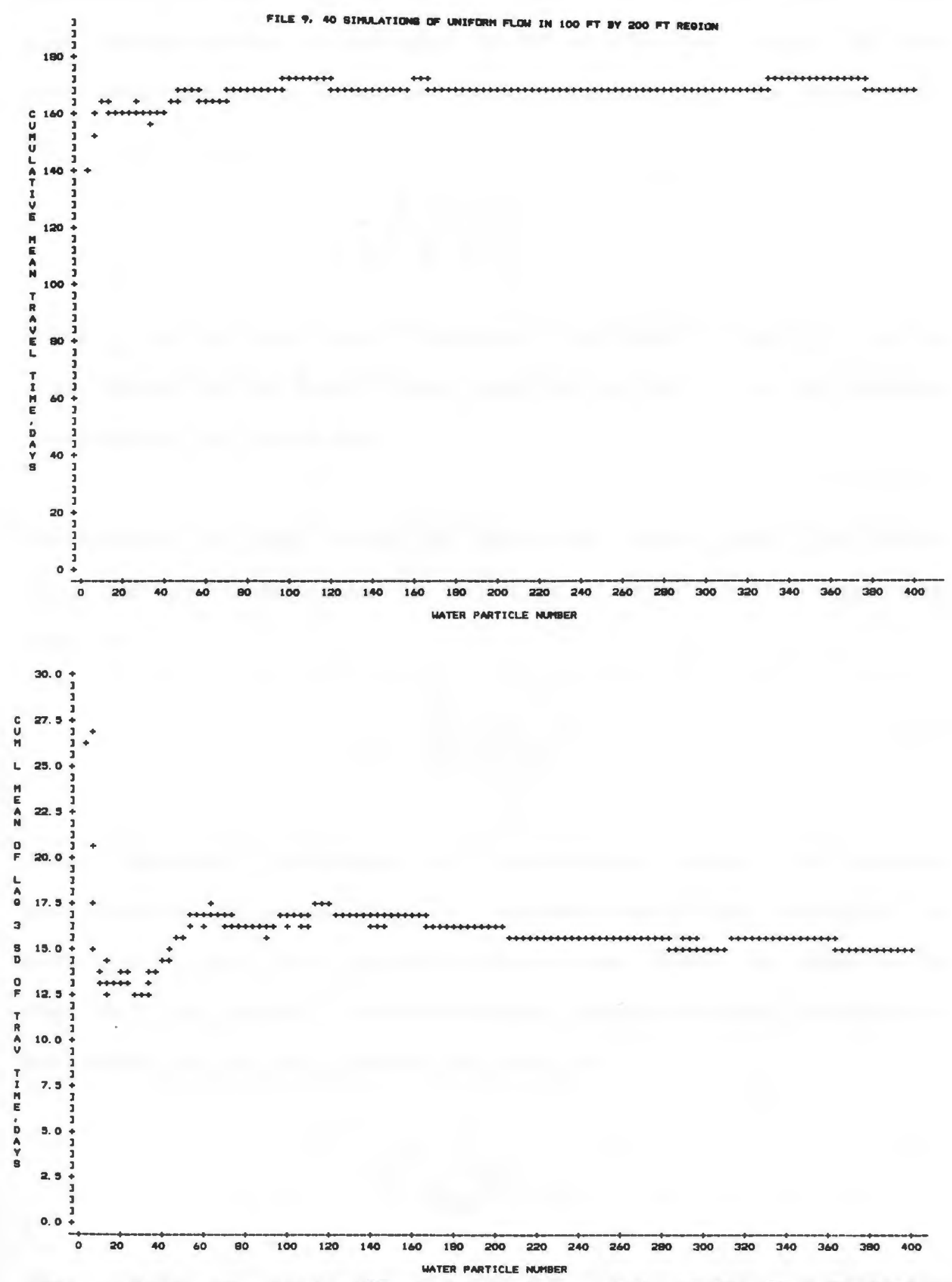

Figure B.21 - Cumulative Mean of the Mean and Lag 3 Standard Deviation of Travel Time Versus Particle Number 
coefficient is directly related to the variance of the travel times. Here concentrations are simulated by the breakthrough curve. The equation developed for a large travel distance relative to the dispersivity is :

$$
D_{L}=\frac{U^{2}\left(2 \sigma_{t}\right)^{2}}{8 t_{0.5}}
$$

where $D_{L}$ is the longitudinal dispersion coefficient, $U$ and $t_{0.5}$ are the mean velocities and travel time respectively, and $\sigma_{t}$ is the standard deviation of the travel time.

The distance, $x$, over which the dispersion occurs equals the product $\mathrm{Ut}_{0.5}$ and may be substituted in this equation resulting in the following equation:

$$
\mathrm{D}_{\mathrm{L}}=\frac{\sigma^{2} \mathrm{x}^{2}}{2\left(\mathrm{t}_{0.5}\right)^{3}}
$$

These equations illustrate the relationship between the standard deviation of the travel time, $\sigma_{t}$, and the longitudinal dispersion coefficient. Since the dispersion coefficient equals the dispersivity times the mean velocity, the macroscopic dispersivity may be determined by dividing by the mean velocity to arrive at:

$$
\delta=\frac{\sigma_{t} x}{2\left(t_{0.5}\right)^{2}}
$$

These results and others indicate that the 400 water particles simulated is sufficient to compute mean and standard deviation for travel time. 
The macroscopic or field scale lateral dispersion coefficient, $\mathrm{D}_{\mathrm{T}}$, has been shown by Fried (1975) to be a function of the scatter about the mean ending location after traversing a region. Fried presented the following equation:

$$
D_{T}=\frac{1}{2}{ }_{y}^{2} \frac{\bar{U}}{x}
$$

where $\sigma_{y}$ is the standard deviation of the normalized location perpendicular to the direction of flow and $\bar{U}$ and $x$ are as defined earlier.

A record was maintained of the starting and ending y coordinate of each water particle. A normalized ending $y$ coordinate was then computed along with a cumulative mean and cumulative mean of the standard deviation as each new record of water transport was added. The same was computed for the $y$ coordinate at the estimated time to transport half the region. A typical plot of these results is presented in Figure B.22. These results also indicate that 400 particles are sufficient to reach a consistent mean value for these variables.

\section{B.8.5 Normality Check on Travel Times}

The ensemble of travel times was utilized to plot a breakthrough curve. Figure B.23 presents a representative sample of a breakthrough curve. These curves are similar to a concentration versus time curve and their similarity of shape supports the premise of general conformance of normality. 

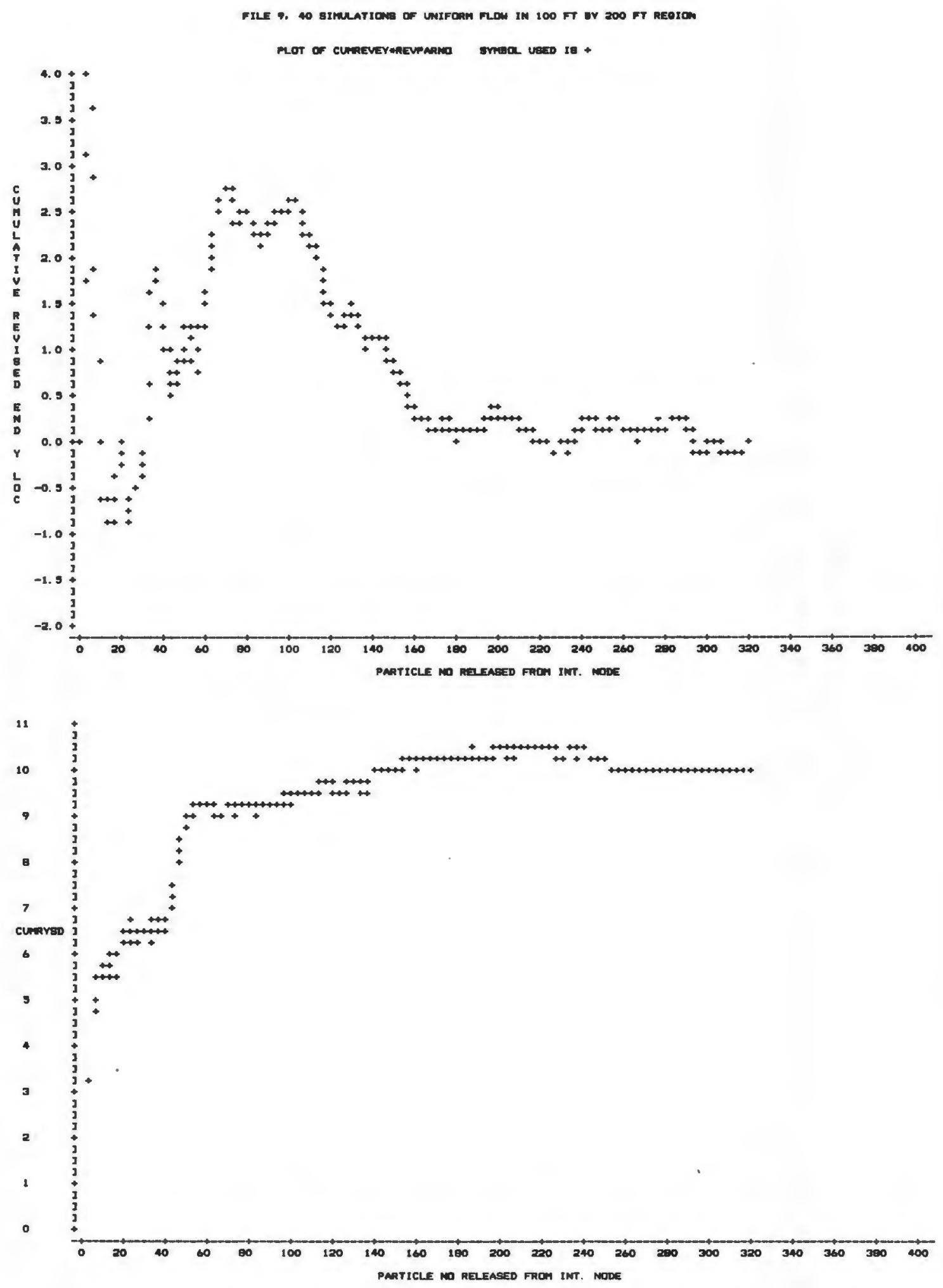

Figure B.22 - Cumulative Mean of the Mean and Lag 3 Standard Deviation of Normalized Ending Y Location 
FILE 9, 40 GIMULATIONB OF UNIFOAM FLOW IN 100 FT OY 200 FT REOIUN

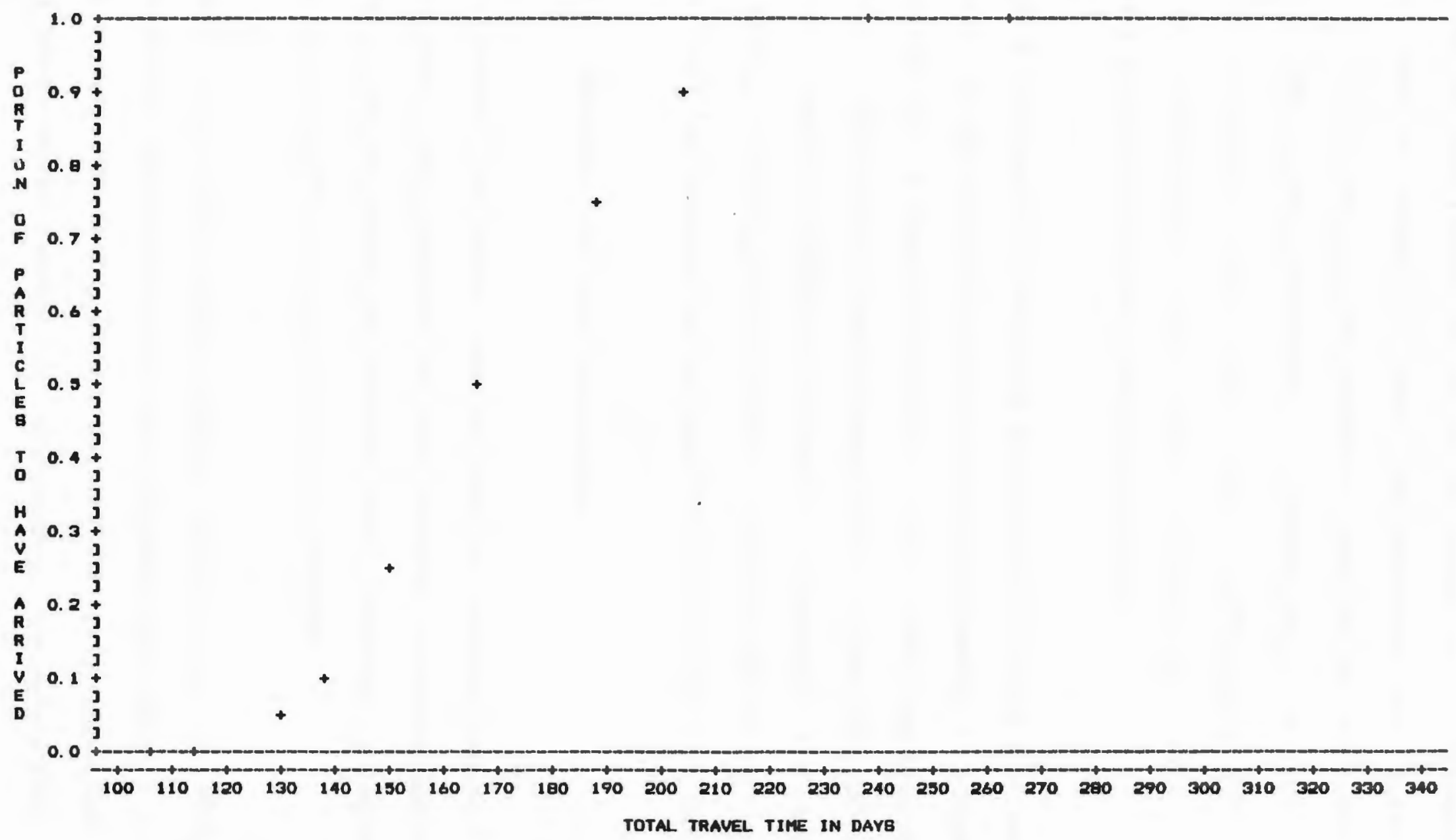

Figure B.23 - Simulation Breakthrough Curve 
A check for the goodness of fit of the assumption of a normal distribution for the ensemble of travel time records was made. A SAS program was written to test the null hypothesis that the data could be a random sample from a normal distribution. A Kolomogorov D statistic was computed and compared with critical values. A Kolomogorov statistic was utilized rather than the more common chi square check since it is more easily adapted to continuous uncategorized data.

Table B.1 presents the results of this check for several flow ensembles. Several of the normality checks support the assumption of a normal distribution with a general tendency to do so as the transit distance increases. Cases 10,11 and 12 suggest that as the time interval decreases, there is a greater likelihood of acceptance of a normal distribution. A Kolomogorov D statistic of 0.10 or greater was considered sufficient for acceptance of the assumption of normality for this study.

\section{B.8.6. Hydraulic Head Value Sensitivity}

The hydraulic head value along two rows was tabulated for each of the simulations. This tabulation was then available to allow computation of mean values and variations resulting from variations in region size, hydraulic gradient, conductivity and other factors.

Figure B.24 presents typical results from one of the Monte Carlo simulations. The upper plot presents the mean hydraulic head versus the node number. The averaging over the 40 Monte Carlo simulations results in a fairly smooth straight line relationship. The middle curve 

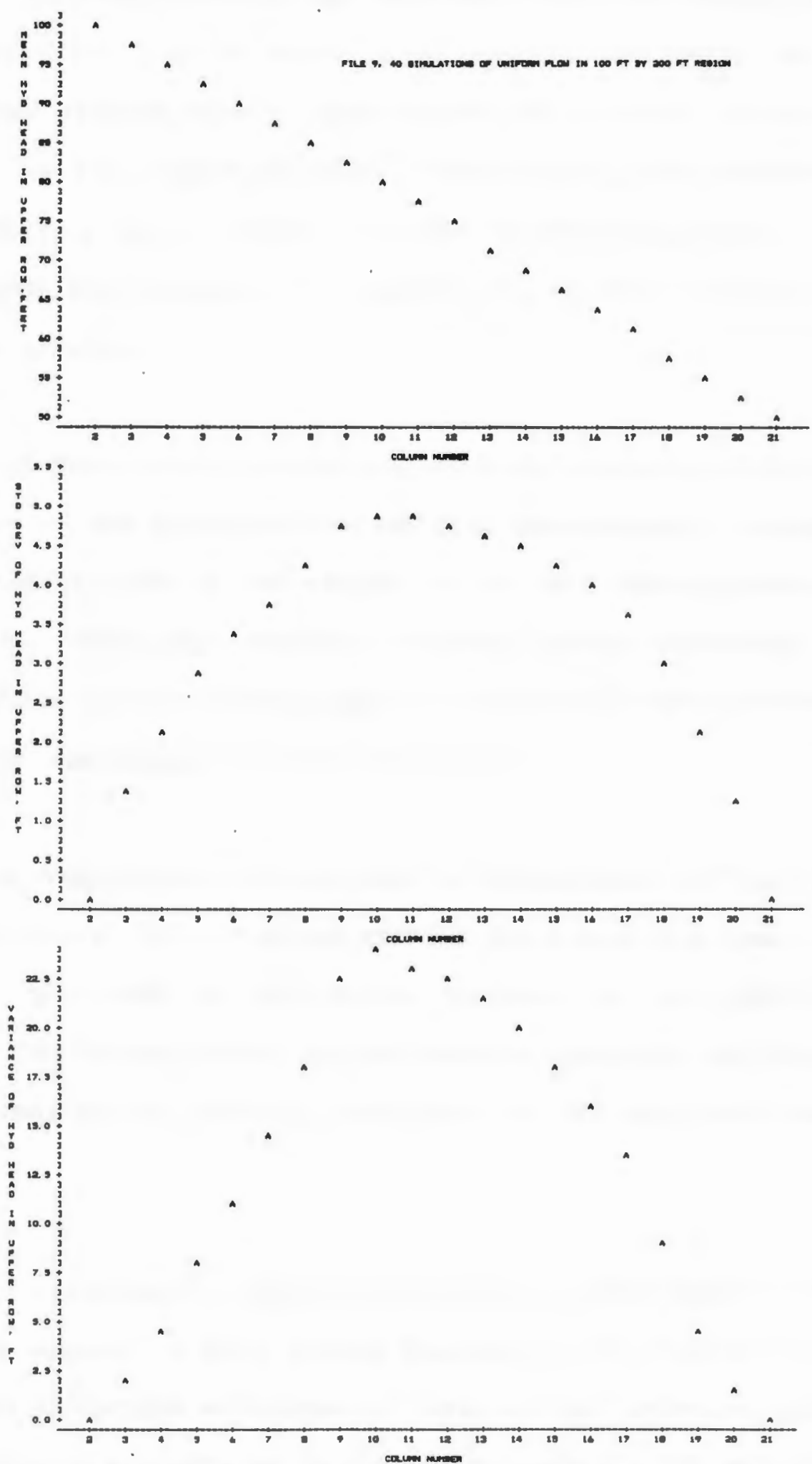

Figure B.24 - Mean, Standard Deviation and Variance of Head Versus Node Number 
presents the standard deviation and the lower curve the variance of the hydraulic head for the 40 Monte Carlo simulations versus the node number. These figures clearly demonstrates the boundary effects. At the ends of the flow region the head is held constant and therefore the standard deviation is zero there. At nodes further away, there is less influence from the boundary and therefore the standard deviation and variance are greater.

The number of Monte Carlo simulations required to reach a fairly consistent value of the hydraulic head was also investigated. Figure B.25 presents a typical plot of the results of one such investigation at an interior node. This plot indicates that the initial simulation had a value very close to the resulting mean value and after three simulations the mean value was within 3 percent of that value.

The effect of variations in region size is demonstrated in Figure B.26. The maximum value of the normalized standard deviation of hydraulic head for any of the nodes in the region increases as the region size increased. The maximum value of the standard deviation of hydraulic head was normalized by dividing each value by the mean head loss for each node.

The effect of variations in hydraulic gradient is demonstrated in Figure B.27. There appears to be a gradual increase in the maximum value of the standard deviation of hydraulic head as the hydraulic gradient increases. This is probably due to the larger numbers in the calculation however, since when divided by the mean value there is no general trend. 
FILE 27, 40 BIMULATIONS OF UNIFORM FLOW IN 100 FT BY 200 FT REGION

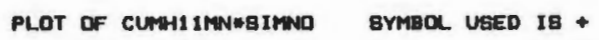

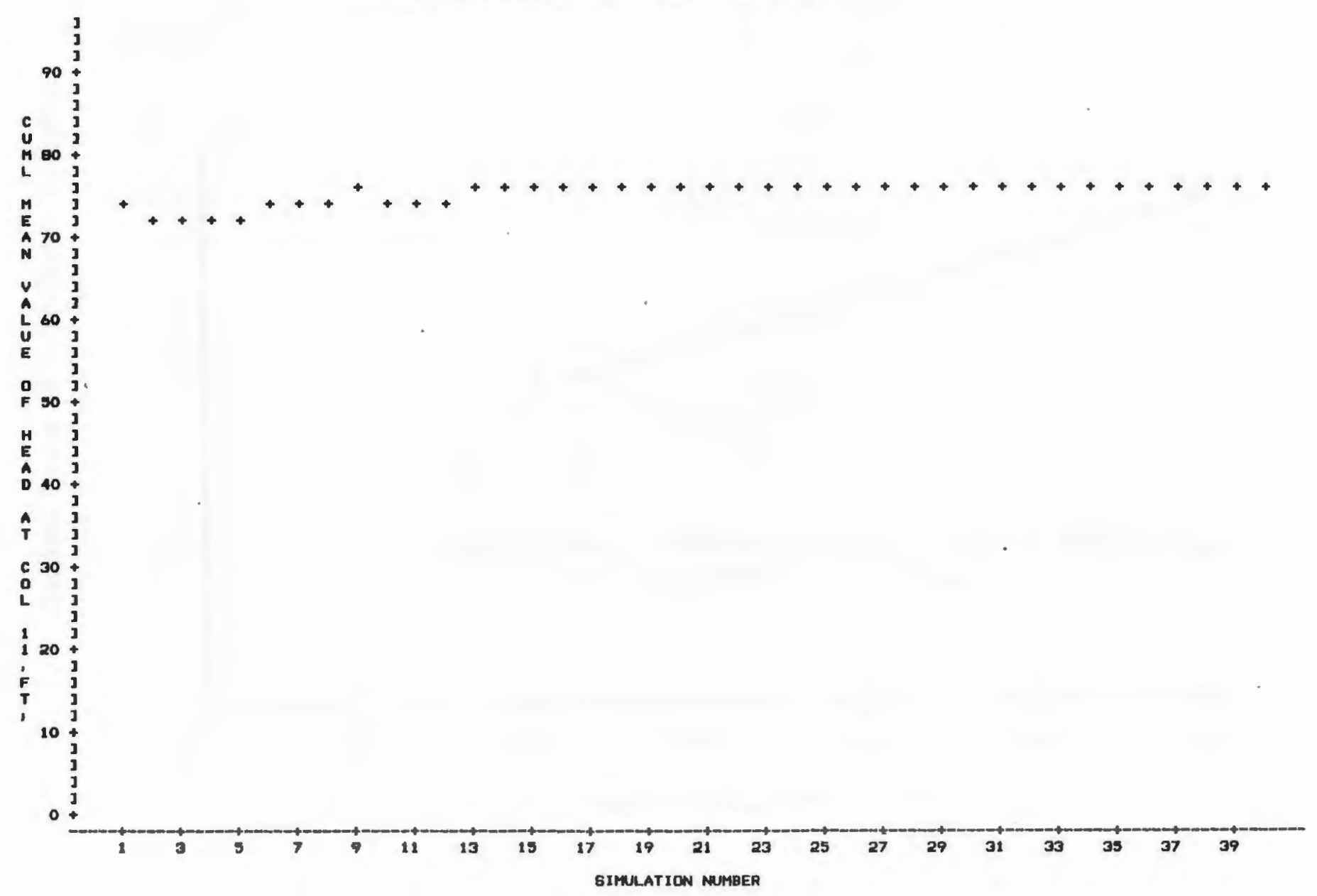

Figure B.25 - Cummulative Mean of the Head Versus Simulation Number 


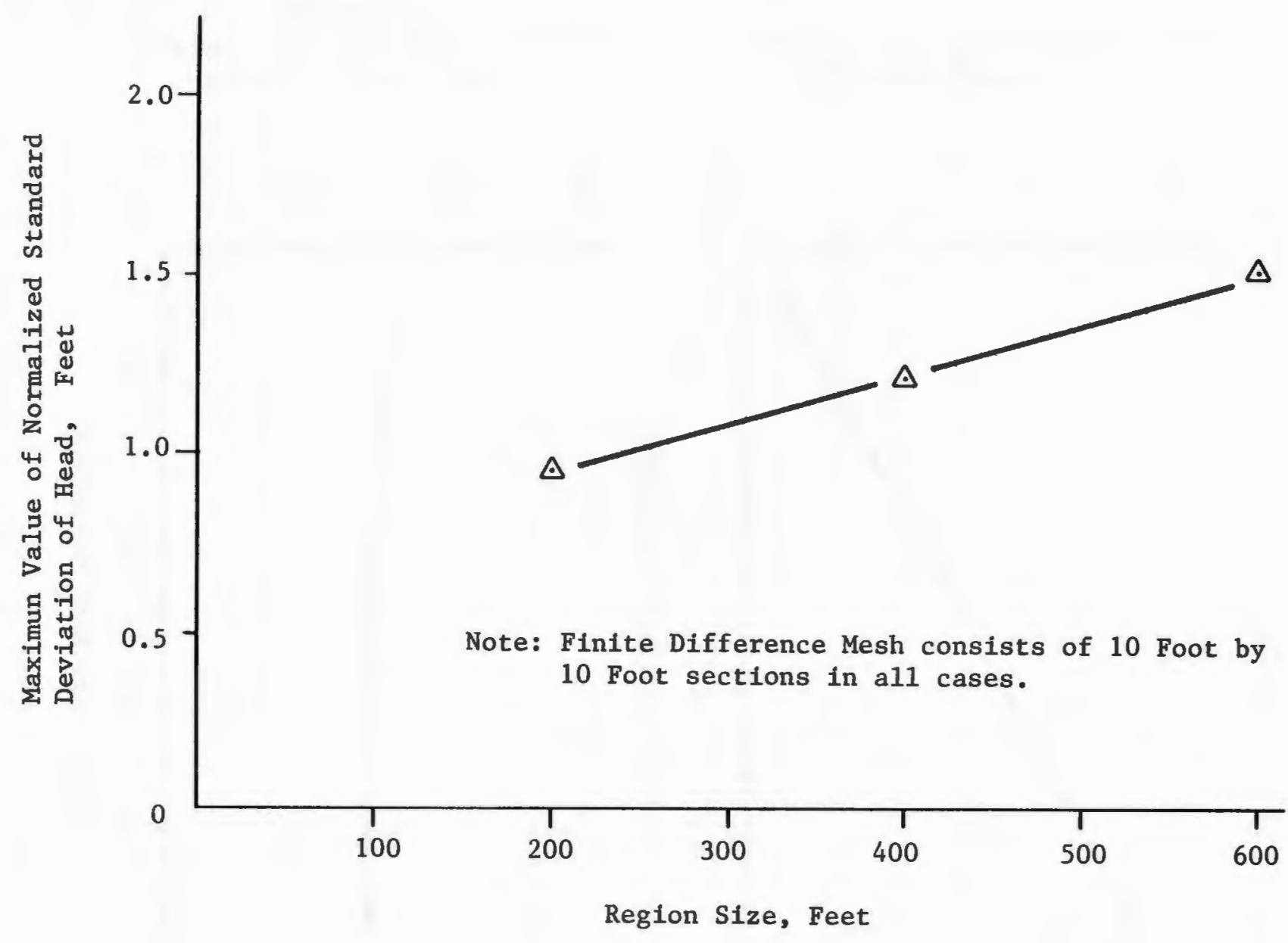

Figure B.26 - Maximum Value of Normalized Standard Deviation of Head Versus Region Size 

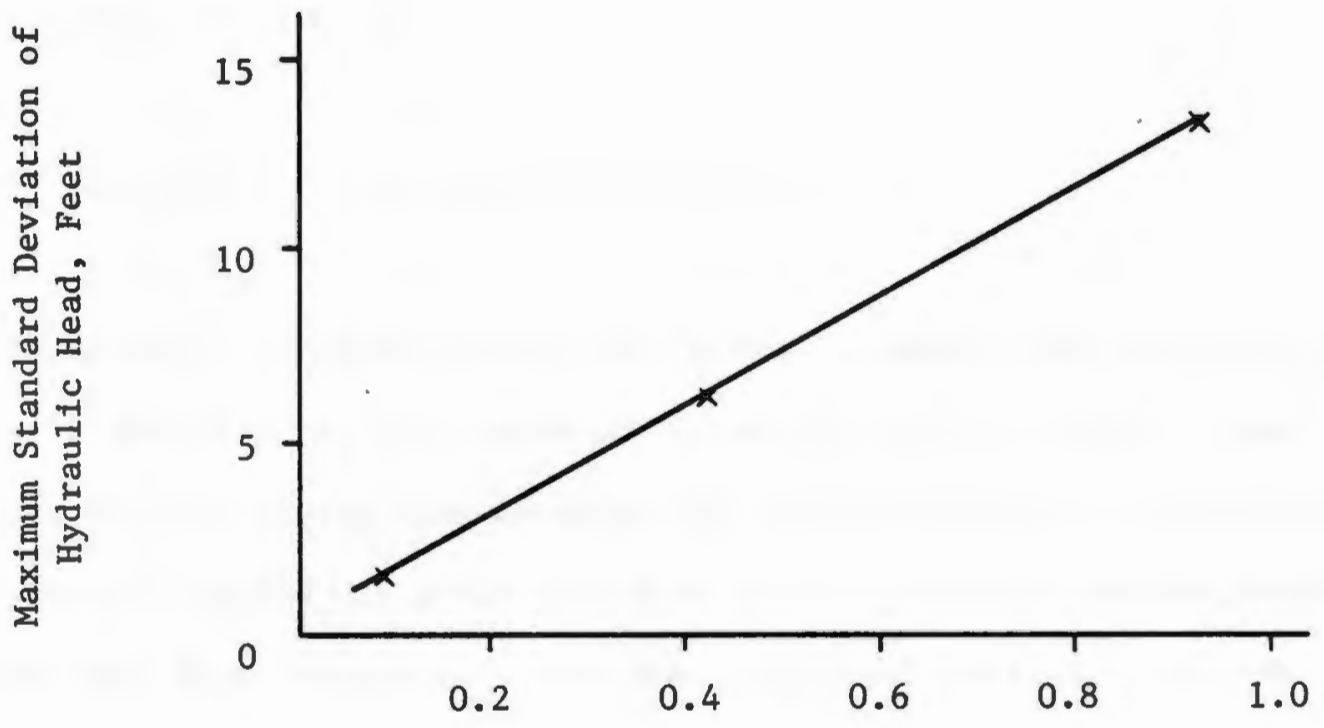

Hydraulic Gradient, Ft/Ft

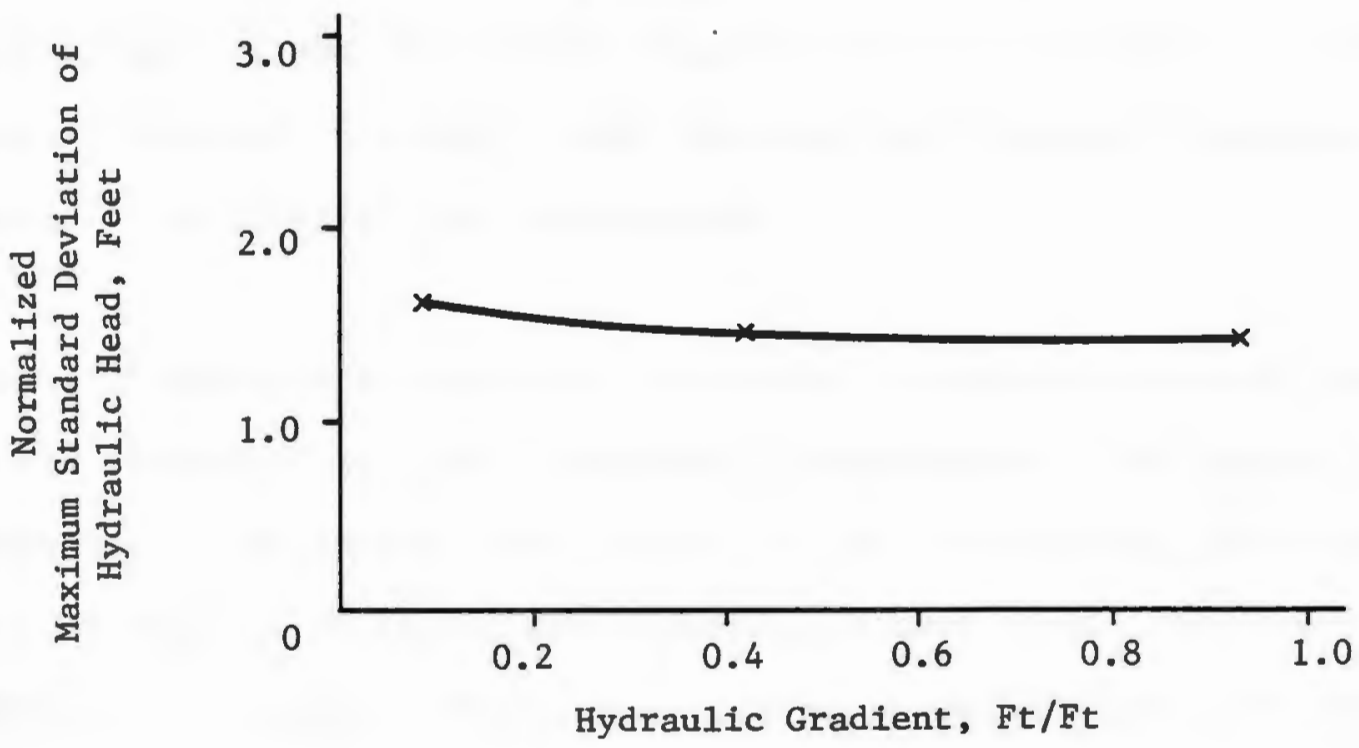

Figure B.27 - Maximum Value of Standard Deviation of Head Versus Hydraulic Gradient 
The relationship between this quantity and the standard deviation of the hydraulic conductivity is of more significance. Figure B.28 presents this relationship which results in a direct relationship. This is significant since the uncertainty in the hydraulic conductivity value directly affects our uncertainty in the value of the hydraulic head value within the region.

\section{B.8.7. Macroscopic Dispersivity Sensitivity}

The macroscopic or field scale longitudinal dispersivity has been shown to be a function of the standard deviation of the travel time. The macroscopic transverse dispersivity has been shown to be a function of the standard deviation about the mean ending location perpendicular to the general flow direction. For the horizontal uniform flow case this may be taken as the standard deviation in the ending $y$ coordinate. The sensitivity of the macroscopic dispersivity to variations in region size, hydraulic gradient, time interval and standard deviation of hydraulic conductivity was investigated.

Figure B.29 presents results of the effect of region size on the microscopic longitudinal and transverse dispersivity. The upper plot indicates a relatively small change in the longitudinal dispersivity with an increase in region size and no consistent trend. The lower plot indicates a steadily decreasing transverse dispersivity with region size. The values plotted for the macroscopic dispersivities was determined by dividing the dispersion coefficients, determined by the equations taken from Fried (1975) given earlier, by the mean particle 


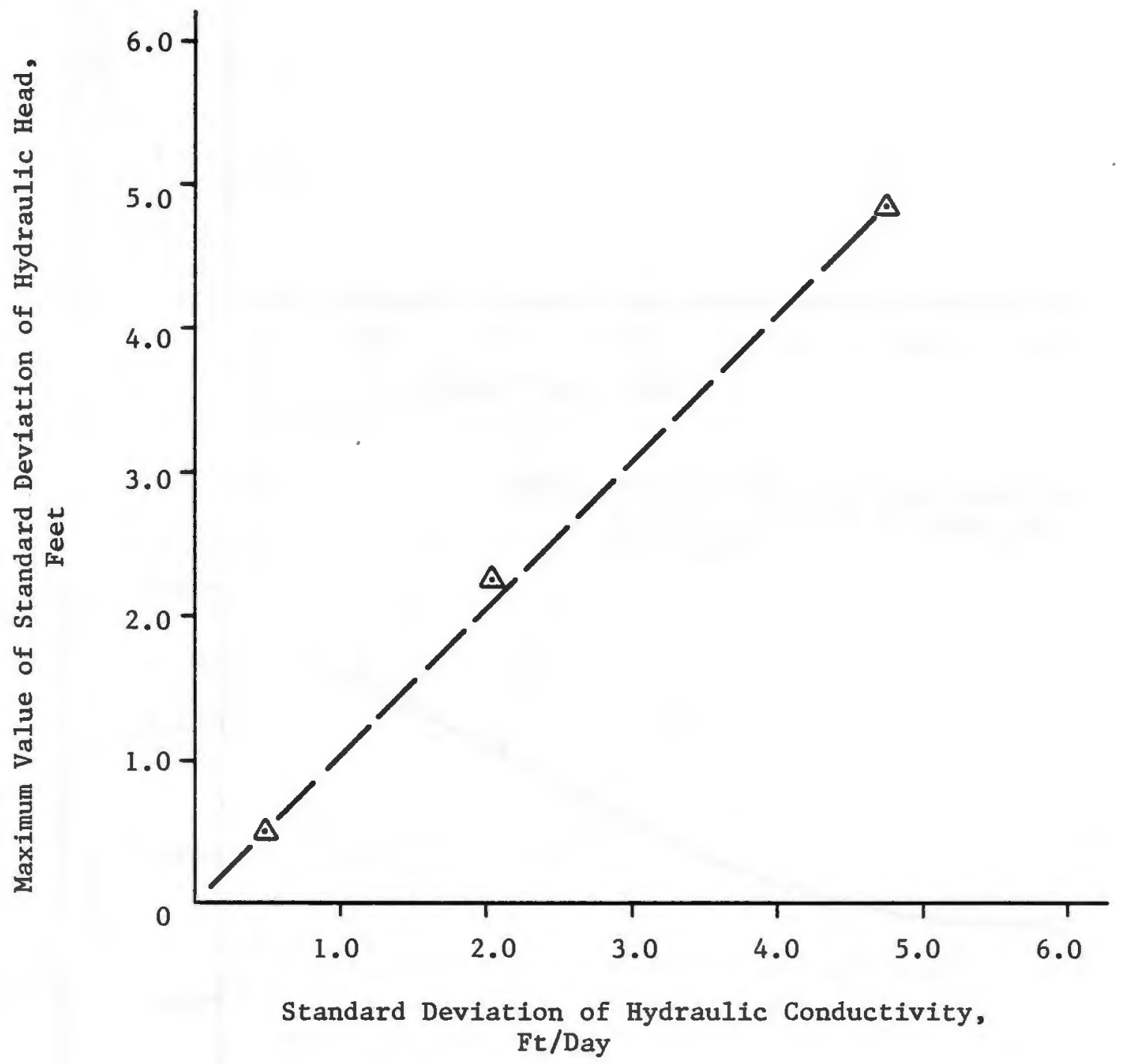

Figure B.28 - Maximum Value of Standard Deviation of Head Versus Standard Deviation of Hydraulic Conductivity 


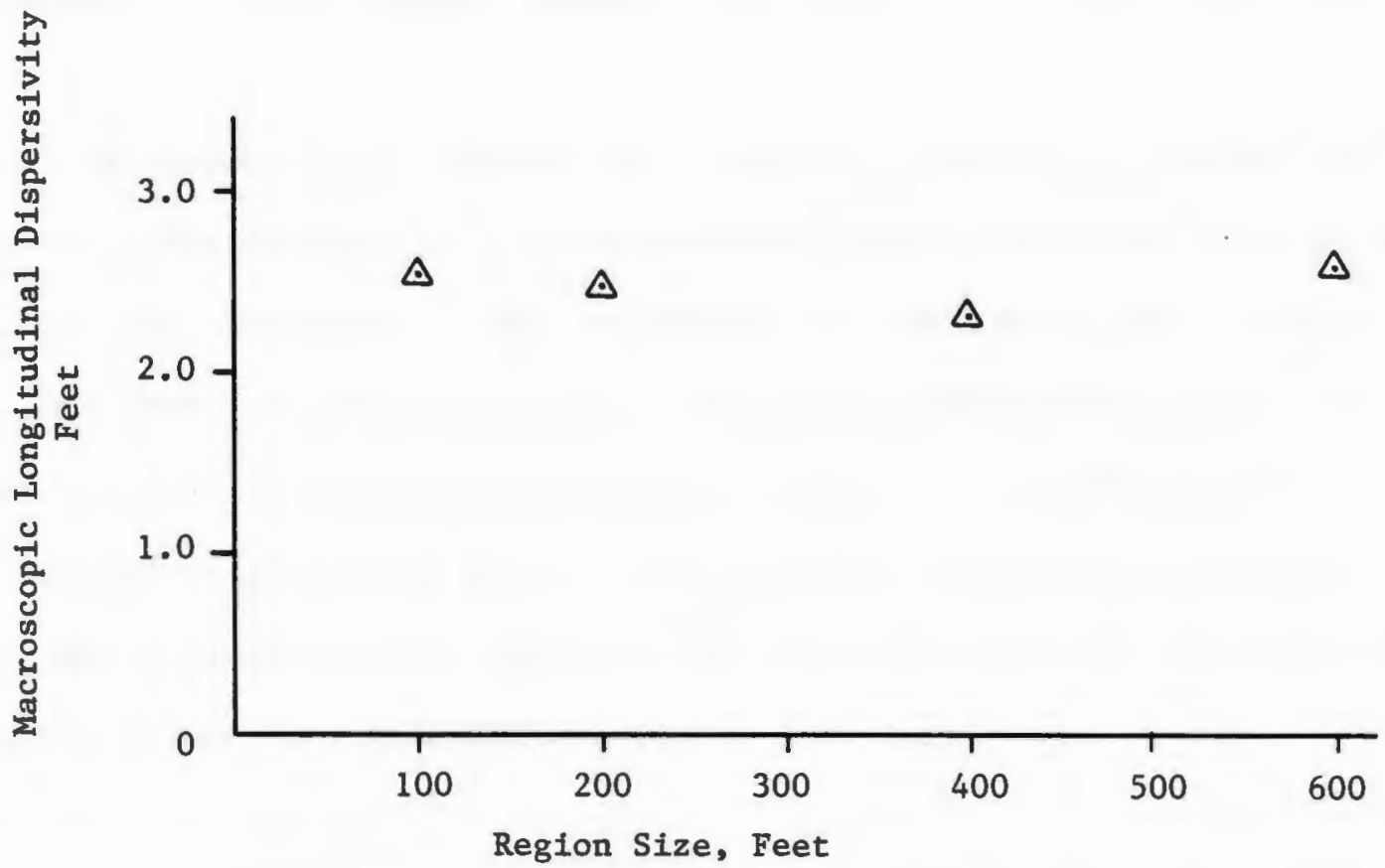

Note: Finite Difference Mesh consists of $10 \mathrm{Ft}$ by $10 \mathrm{Ft}$ Sections in all Cases.

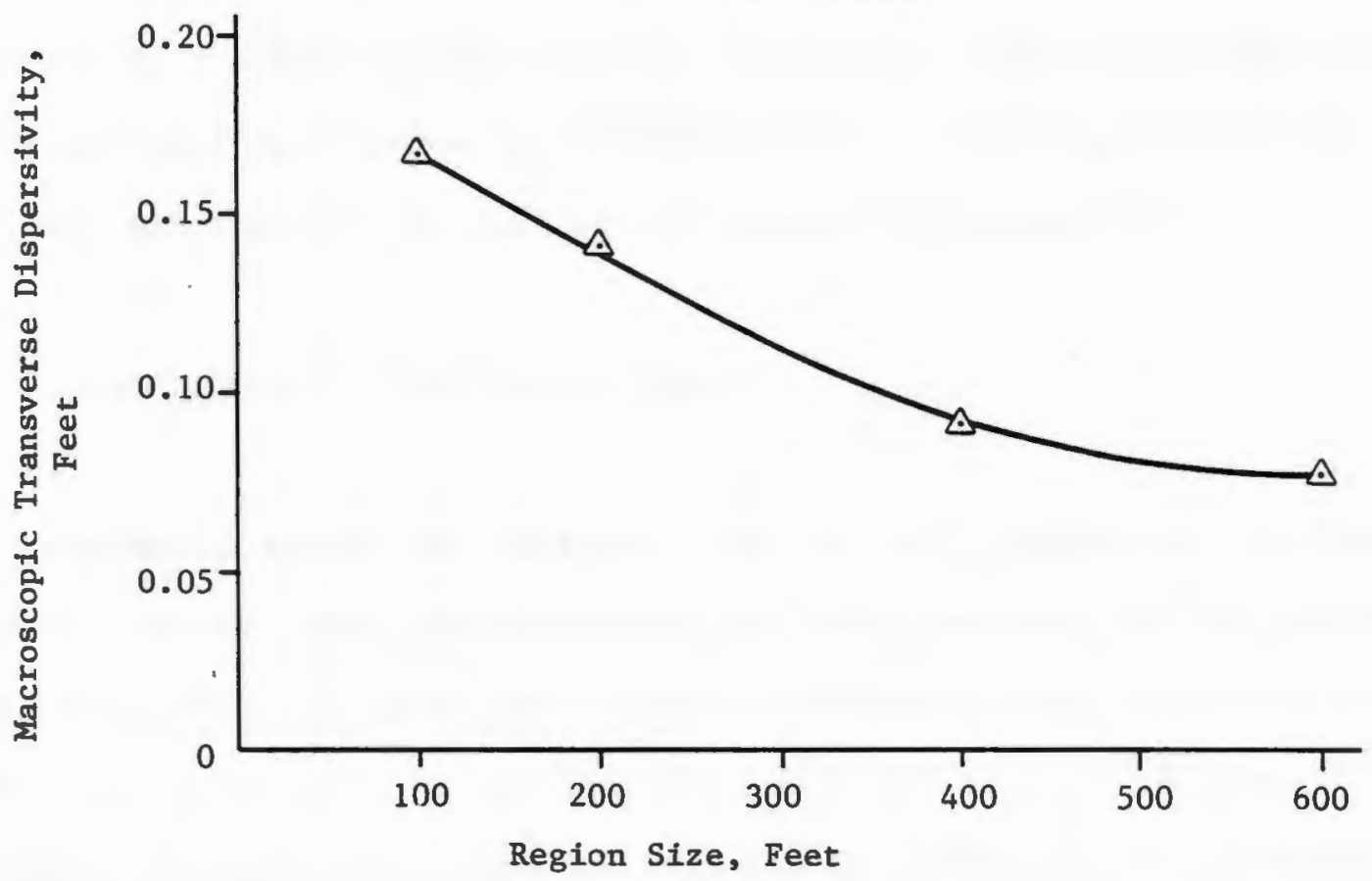

Figure B.29 - Macroscopic Dispersivity Versus Region Size 
velocity in the region. These results suggest that a constant value of dispersivity is not reached during the transit of these particles.

Figure B.30 presents the effects of a change in hydraulic gradient and Figure B.31 the effects of a change in time interval for mass transport on these same parameters. The variation of both macroscopic longitudinal and transverse dispersivity is small and inconsistent due to the change in hydraulic gradient and time interval. The insensitivity of the observed dispersivity due to time interval variation is important since the computer time required to simulate particle transit is dependent on the time interval utilized.

The effect of a variation in the standard deviation of hydraulic conductivity is much more pronounced than these other variables. The results of a variation of this parameter are presented in Figure B.32. A reduction in standard deviation of the hydraulic conductivity from 2.0 to 0.5 resulted in a reduction from 2.5 to 0.3 in the longitudinal dis persivity and from 0.14 to 0.05 in the transverse dispersivity.

\section{B.8.8. Development of Confidence Limits}

The confidence which the engineer has in his prediction of flow quantity, travel times and dispersion of flow particles is the major reason for utilizing these more sophisticated and complex solutions for field flow problems. If the engineer were not able to increase his confidence in his predictions there would be little reason to utilize these techniques. The nature of the output from this method lends 
南

$\triangle$

$\triangle$

$\Delta$

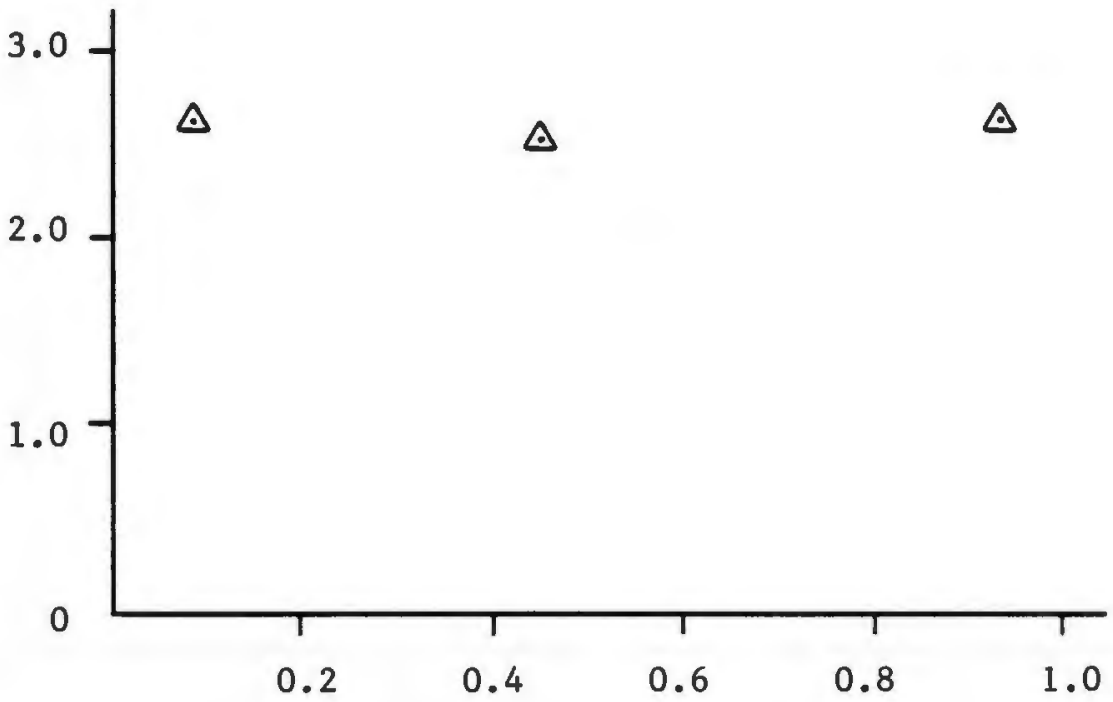

Hydraulic Gradient, Ft/Ft

年

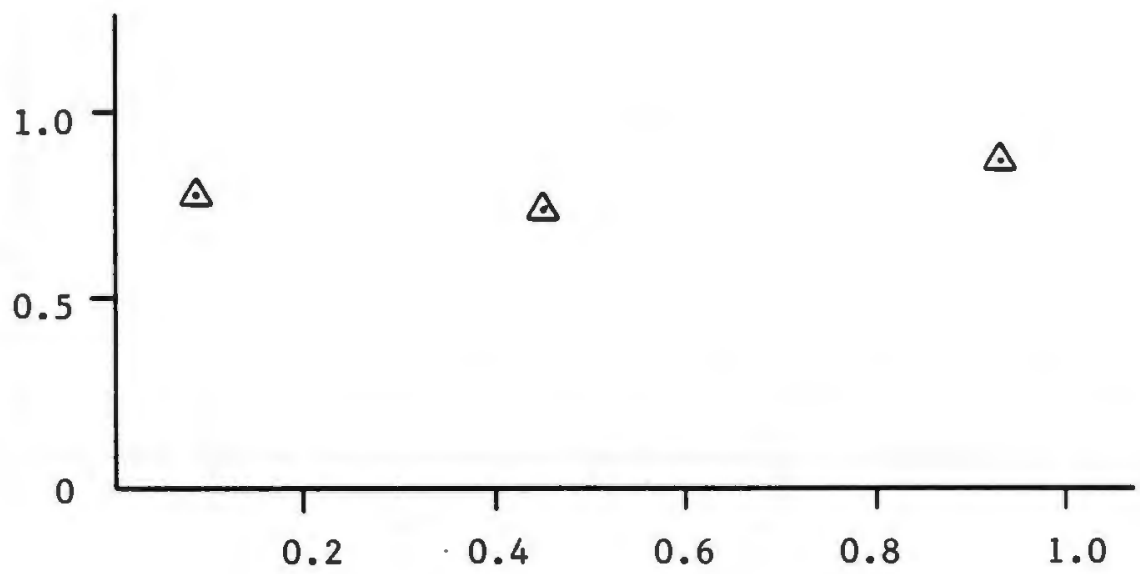

Hydraulic Gradient, Ft/Ft

Figure B.30 - Macroscopic Dispersivity Versus Hydraulic Gradient 
ث્1

$\Delta$

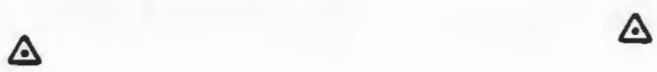

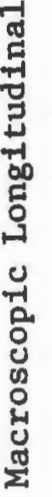

2.0

过

fra

$1.0-$

0

\begin{tabular}{llllll}
\hline & 1 & 1 & 1 & 1 \\
1.0 & 2.0 & 3.0 & 4.0 & 5.0 & 6.0
\end{tabular}

Time Interval, Days

मे

$\Delta$

$\Delta$

$\Delta$

0
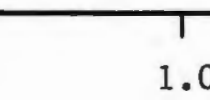

.02 .0

3.0

4.0

5.0

6.0

Time Interval, Days

Figure B.31 - Macroscopic Dispersivity Versus Time Interval 
itself to the development of confidence limits on the solution.

A methodology to utilize the knowledge of the variability of the flow region characteristics to establish confidence limits on the output utilizing probability and statistics has been formulated. The emphasis of this methodology is in the quantification of the characteristics of the major input parameter, hydraulic conductivity. Other parameters have been shown to be of lesser importance to the problem solution.

The procedure to develop the site specific soil parameters is essentially unchanged. Field programs are utilized to measure insitu soil parameters and to secure samples for laboratory testing . The field and laboratory tests will be analyzed so that a probability density function of the hydraulic conductivity may be formulated. Other parameters such as porosity, and laboratory dispersivity characteristics must also be developed.

These parameters are then the input to a Monte Carlo computer solution for flow quantity and travel time. The results of these Monte Carlo computer simulations may then be analyzed utilizing the knowledge of the underlying distribution of these results to develop confidence in the results.

In general the flow quantity and travel time results should be expected to conform to a normal distribution. This assertion is made on the basis of the Central Limit Theorem. The water particle movement may be considered the result of adding up the effects of many individual 


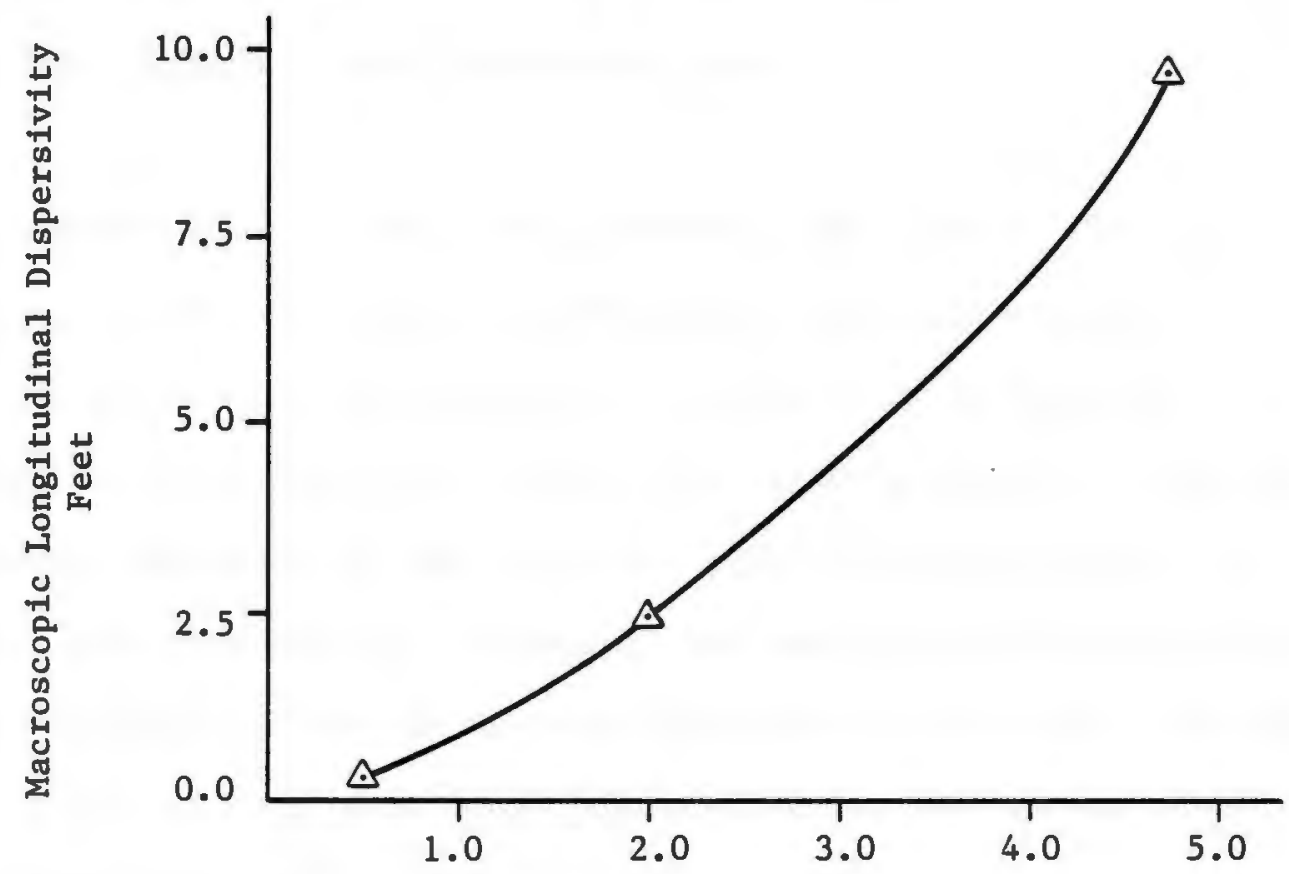

Standard Deviation of Hydraulic Conductivity, ft/day

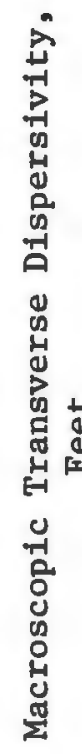

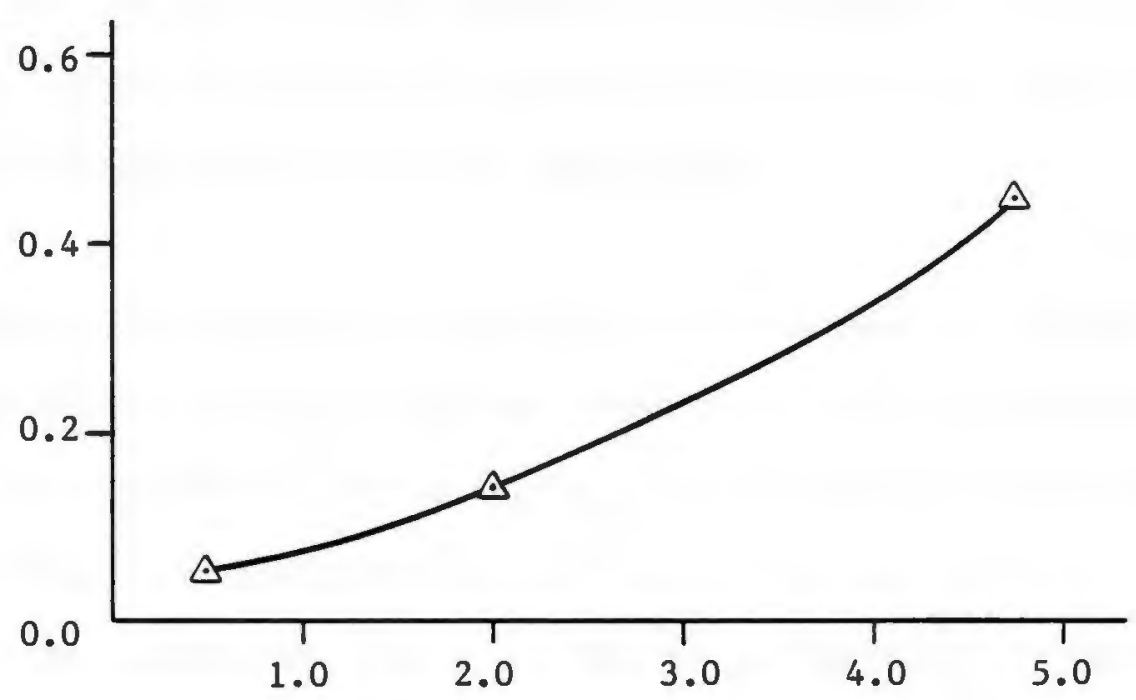

Standard Deviation of Hydraulic Conductivity, ft/day

Figure B.32 - Macroscopic Dispersivity Versus the Standard Deviation of Hydraulic Conductivity 
factors (hydraulic conductivity values). This assumption of normality has been generally verified in this study.

The mean value of the flow quantity and travel time may then be estimated with confidence by the method described earlier. The results of one Monte Carlo simulation are presented in Figure B.33. The characteristics of the flow region are also presented. The mean and standard deviation of the effective hydraulic conductivity, $\mathrm{K}_{\mathrm{q}}$, is 4.57 and 0.230 respectively. Utilizing the equations developed earlier, the $95 \%$ confidence limits on the mean value may be estimated. The magnitude of error of the mean with $95 \%$ confidence may be estimated by the following:

$$
E<t_{\alpha / 2} \cdot \frac{s}{\sqrt{N}}=1.96 \cdot \frac{0.230}{\sqrt{40}}=0.071
$$

where $t_{d / 2}$ is the value of the cumulative distribution function of the student $t$ distribution at the $\alpha / 2$ significance level, $s$ is the value of the standard deviation and $\mathrm{N}$ is the sample size.

The mean value would then be expected to lie between the limits of the sample mean (4.57) plus or minus the error (0.07) i.e., 4.50 and 4.64 . The effective hydraulic conductivity, $\mathrm{K}_{\mathrm{q}}$, was calculated by dividing the flow by the mean hydraulic gradient and area. The flow rate may then be estimated with confidence limits of $95 \%$ by multiplying by these constants. In so doing the flow limits of 38.1 to $39.9 \mathrm{ft}^{3} /$ day were estimated with $95 \%$ confidence limits. 

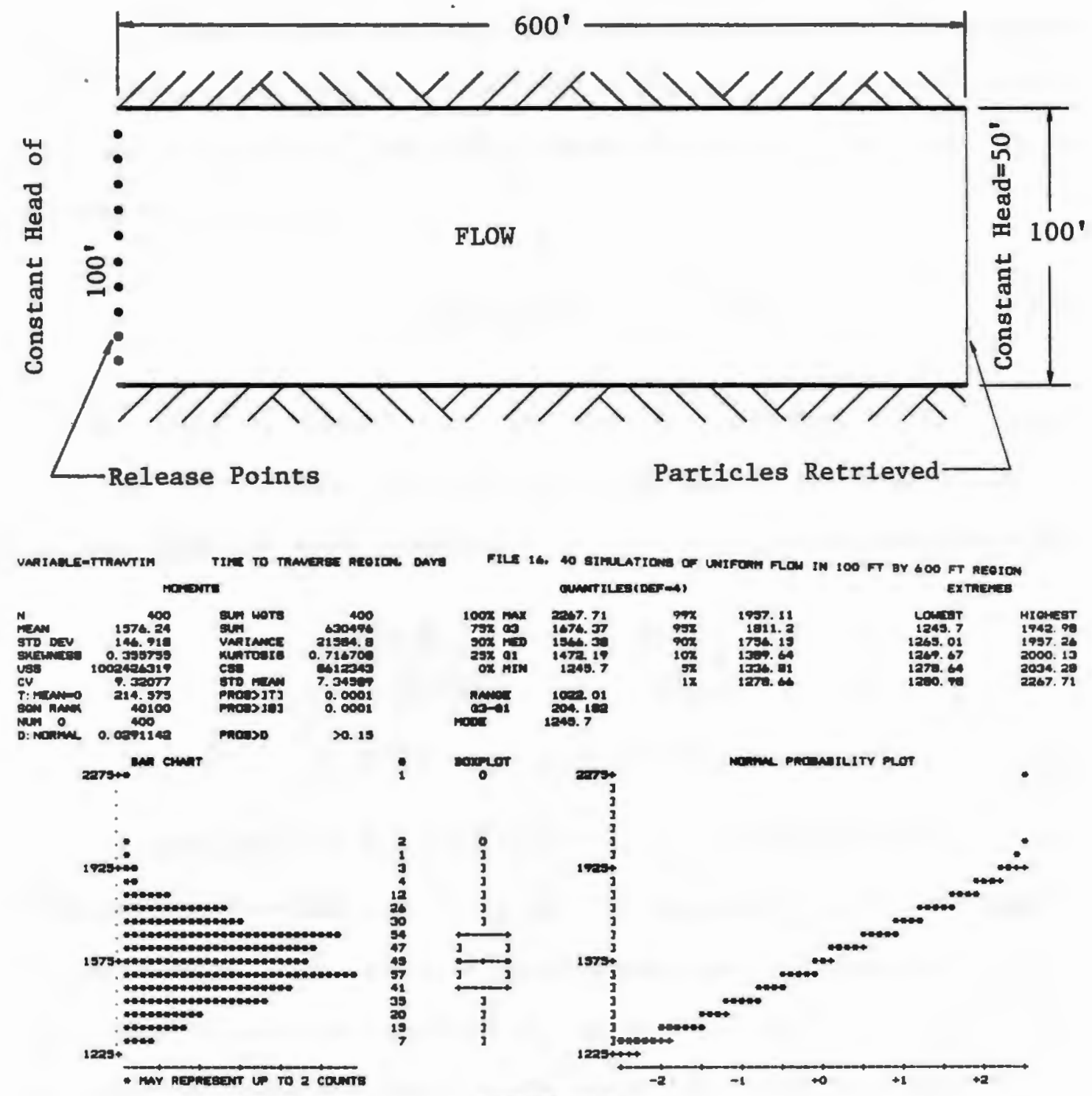

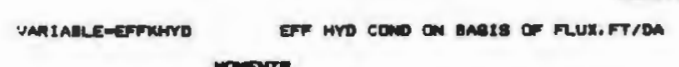
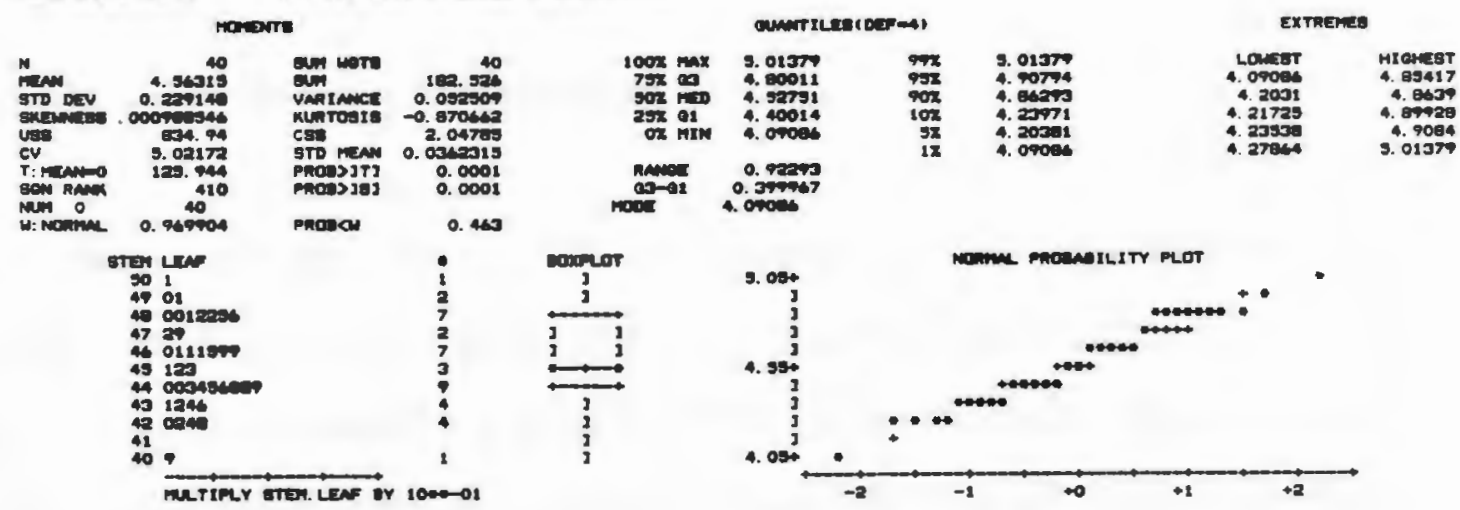

Figure B.33 - Flow Region and Typical Output of Effective Hydraulic Conductivity (Flux) and Travel Time 
The same methodology may be utilized to develop confidence limits on the travel times. The mean and standard deviation of the travel time is 1576.2 and 146.9 days. The $95 \%$ confidence limits on the magnitude of error may be estimated as:

$$
E<\frac{1.96(146.9)}{\sqrt{400}}=14.4 \text { days }
$$

The mean value of travel time is therefore expected to lie between 1561.8 and 1590.6 days. The standard deviation of the travel time may also be estimated with confidence by the expressions given earlier.

$$
\begin{aligned}
& \frac{146.9}{1+\frac{1.96}{\sqrt{2(400)}}}<\sigma<\frac{146.9}{1-\frac{1.96}{\sqrt{2(400)}}} \\
& \therefore \quad 137.4<\sigma<157.8
\end{aligned}
$$

The water particles are expected to follow a normal distribution so that a hypothetical breakthrough curve may be developed for the extremes of these parameters. The result of such an exercise is presented in Figure B.34. This allows the engineer to estimate not only the mean arrival time with confidence but also the distribution of travel times.

\section{B.8.9 Macroscopic Dispersivity}

The results of the various flow situations was analyzed for characterizations of macroscopic dispersion coefficients and dispersivity. Using the equation developed by Fried (1975), a macroscopic dispersion coefficient was calculated for the record of 400 flow particle traverses of the flow region. The equation developed by Fried assumes that the 


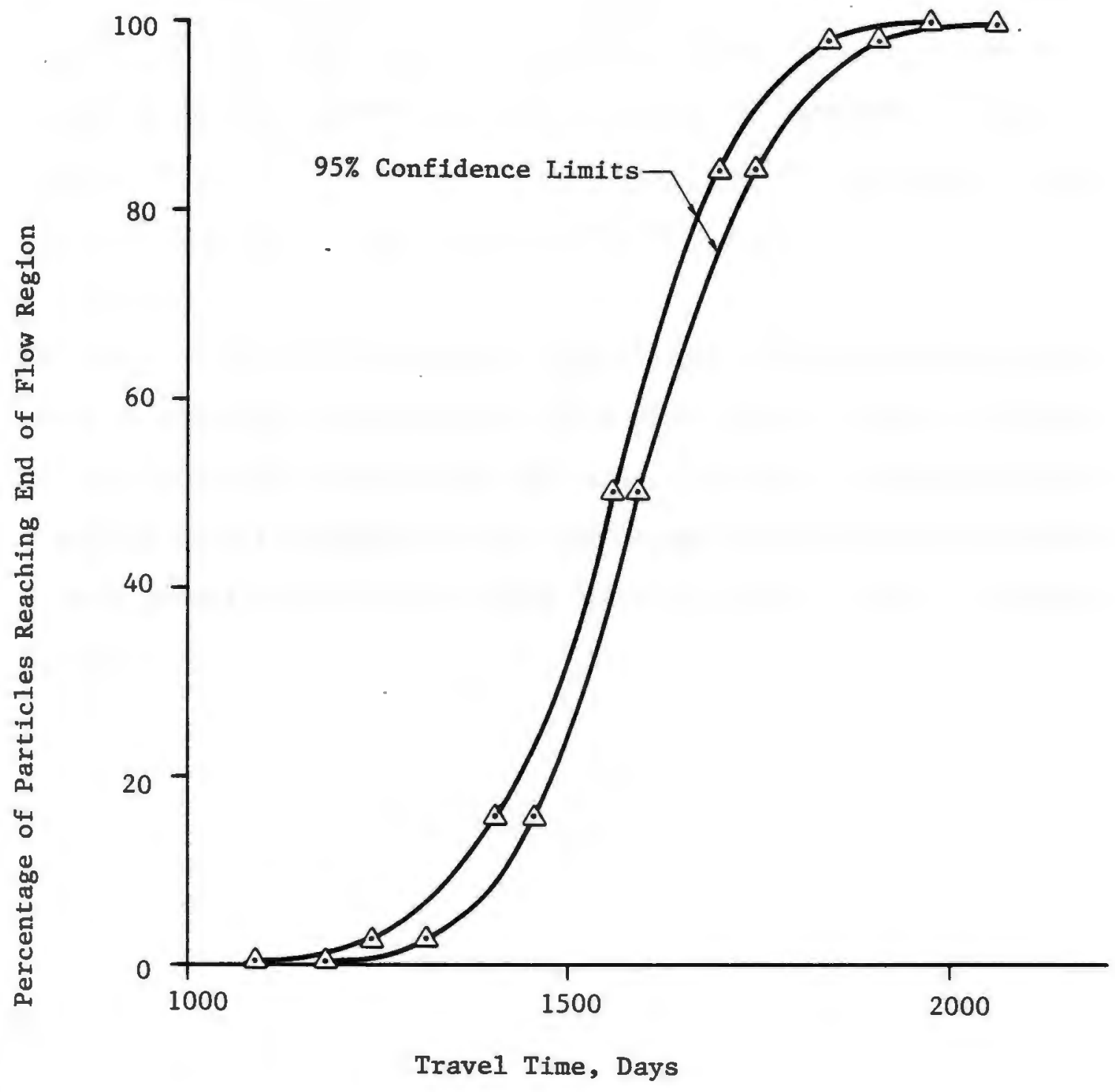

Figure B.34 - Hypothetical Breakthrough Curve 
particle breakthrough curve will simulate flow concentration. This macroscopic dispersion coefficient and dispersivity was plotted versus the mean region velocity and is presented in the upper two curves of Figure B.35. The upper curve indicates a general increase in the macroscopic dispersion coefficient with velocity as expected. There is no general trend for the middle curve. The value of macroscopic dispersivity appears not to vary greatly with velocity.

The lower curve plots macroscopic dispersivity versus the standard deviation of hydraulic conductivity. This plot suggests that as the variation in hydraulic conductivity increases, the value of macroscopic dispersivity should increase. This trend suggests that particle transport is less direct as the variation of hydraulic conductivity in the region increases. 

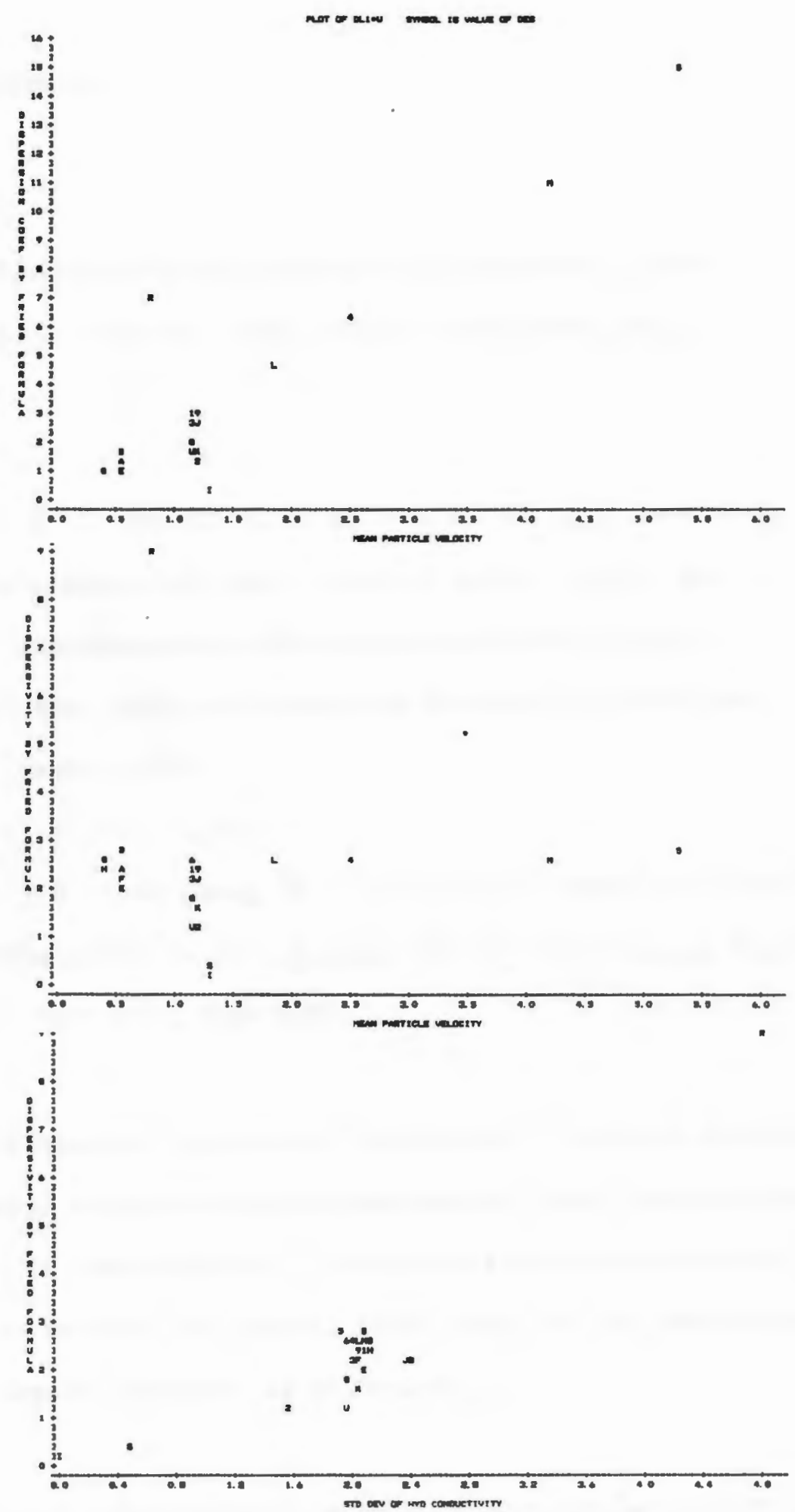

Figure B.35 - Macroscopic Dispersion Coefficient and Dispersivity Versus Mean Velocity and Dispersivity Versus Standard Deviation of Hydraulic Conductivity 
APPENDIX C - BIBLIOGRAPHY

1. Agterberg, F. "Autocorrelation Functions in Geology" in Geostatistics, D. Merriam, edit, Plenum Press, New York, p 113$142,1970$.

2. Ahlstrom, S.W., H. P. Foote, R. C. Arnett, C. R. Cole, and R. J. Serne, "Multicomponent mass transport model: Theory and numerical implementation (discrete-parcel-random walk version)," Rep. BNWL-2127, Battelle Pacific Northwest Lab., Richland, Wash., 1977.

3. Ali, E.M., Wu, T.H., and Chang, N.Y., "Stochastic Model of Flow Through Stratified Soils", Journal of the Geotechnical Division, ASCE, Vol. 106, GT6, June 1980.

4. Bakr, A.A., "Stochastic analysis of the effect of spatial variations in hydraulic conductivity on groundwater flow," dissertation presented to the New Mexico Institute of Mining and Technology at Socorro in 1976, in partial fulfillment of the requirements for the degree of Doctor of Philosophy.

5. Bartlett, M.S., The Statistical Analysis of Spatial Pattern, 91 pp., Chapman and Hall, London, 1975. 
6. Bear, J., "Some Experiments in Dispersion", J. Geophys. Res., Vol. 66 , No. 8, p. $2455-2467,1961$.

7. Bear, J., Dynamics of Fluids in Porous Media, p. 764, Elseview, New York, 1972.

8. Bendat, J.S. and Piersol, A.G., Random Data: Analysis and Measurement Procedures, John Wiley and Sons, Inc., New York, New York, 1971.

9. Benjamin, J.R., and Corne11, C.A. Probability Statistics and Decision for Civil Engineers, McGraw Hill, New York, 1970.

10. Besag, J., "Spatial interaction and the statistical analysis of lattice systems", J. Roy. Statist. Soc., B 36, 192-237, 1974.

11. Bouwer, H., "Planning and interpreting soil permeability measurements," J. Irrig. Drain Div., ASCE, Vol. 95, 391-402, 1969.

12. Brook, D., "On the Distinction between the conditional probability and the joint probability approaches in the specification of nearest neighborhood systems," Biometrika, Vol. 51, 481-483, 1964.

13. Butler, Stanley S., and Grundlach, David L., "Discharge and Travel Time for Ground-Water Conduits" J. of the Irrigation and Drainage Division, ASCE, Vol. 100, No. IR1, Mar. 1974. 
14. Casagrande, Arthur, "Seepage Through Dams", Journal of the New England Water Works, Vol. 51, Jun. 1937.

15. Cedergren, H.R., Seepage, Drainage and Flow Nets. New York: John Wiley and Sons, Inc., 1967.

16. Corps of Engineers, "Big River Reservoir Project, Rhode Island," Vol. III, Appendix F - Geotechnical Analysis, July, 1980.

17. Darcy, H., "Les Fontaines Publiques de la ville de Dijon", Victor Dalmont, Paris, 1856.

18. Freeze, R.A. "A stochastic-conceptual analysis of one dimensional groundwater flow in nonuniform homogeneous media", Water Resour. Res., Vo1. II (5), 725-741, 1975.

19. Freeze, R.A. and Cherry, J.A., Groundwater, Prentice-Ha11, New Jersey, 604 p, 1979.

20. Freund, John E. and Walpole, Ronald E., Mathematical Statistics, Prentice-Hall, Inc., 1980.

21. Fried, J.J., Groundwater Pollution, Elsevier Scientific, Amsterdam, 330 p. 1975. 
22. Gelhar, L.W., "Effects of hydraulic conductivity variations in ground-waterflows", in Proceedings, Second International IAHR Symposium on Research, Lund, Sweden, 1977.

23. Gelhar, L.W., A. L. Gutjahr, and R. L. Naff, "Stochastic Analysis of Macrodispersion in a Stratified Aquifer", Water Resources Research, Vol. 15, No. 6, Dec. 1979.

24. Grace, John R. "Modeling Density Dependent Flow Through Porous Media" thesis presented to the University of Rhode Island at Kingston, Rhode Island in 1981, in partial fulfillment of the requirements for a Masters degree.

25. Greenkorn, R.A. and Kessler, D.P. "Dispersion in Heterogeneous Nonuniform anisotropic Porous Media" Ind. Eng. Chem., Vol. 61(9), p $14-32,1969$.

26. Gutjahr, A.L., L.W. Gelhar, A.A. Bakr, and J.R. MacMillan, "Stochastic analysis of spatial variability in subsurface flows, 2 , Evaluation and Application", Water Resour. Res,, Vol. 14 (5), p 953-959, 1978.

27. Hampton, D., Yoder, E.J. and Burr, I.W., Variability of Engineering Properties of Brookston and Crosby Soils, Purdue University Reprints, CE 199, July, 1963. 
28. Harr, M.E. Groundwater and Seepage, New York: McGraw-Hill Book Co., Inc., 1962 .

29. Harr, M.E. Mechanics of Particulate Media, A Probabilistic Approach, McGraw-Hill Book Co., Inc., 1977.

30. KAME, a Joint Venture, Rhode Island Water Resources Board, Big River Water Supply Project, Preliminary Geotechnical Investigation, Phase 1, Vol 1-2, Nov. 1977.

31. Kelly, William E., personal communication, 1982.

32. Koch, G.S. Jr., and R.F. Link, Statistical Analysis of Geological Data, Vol. 1 and 2, Dover Publications, Inc., New York, 1970.

33. Krumbein, W.C., "Trend Surface Analysis of Contour-Type Maps with Irregular Control-Point Spacing", Journal of Geophysical Research, Vol. 64, No. 7, July 1959.

34. Krumbein, W.C. and Sloss, L.L. Stratigraphy and Sedimentation, W.H. Freeman and Company, San Francisco, CA, 1964.

35. Lambe, T.W., Soil Testing for Engineers, Wiley, New York, 1951.

36. Law, Jan "A Statistical Approach to the Interstitial Hetergeneity of Sand Reservoirs", Petroleum Engineers by the American Institute of Mining and Metallurgical Engineers, May, 1944. 
37. Leonards, G.A., Editor, Foundation Engineering, McGraw Hil1, 1962.

38. Lumb, P., "The Variability of Natural Soils", Canadian Geotechnical Journal, Vo1. 3, 1966.

39. Lumb, P., "Safety Factors and the Probability Distribution of Strength" Canadian Geotechnical Journal, Vol. 7, 1970.

40. Lumb, P., "Application of Statistics in Soil Mechanics" Chapter 3 in "New Horizons in Soil Mechanics" Ed. by I.K. Lee, American Elsevier Publishing Co. Inc. N.Y., 1974.

41. Lumb, P. and Holt, J.K., "The Undrained Shear Strength of a Soft Marine Clay from Hong Kong", Geotechnique, Vol. 18, 1968.

42. Masch, Frank D. and Denny, Kleber, "Grain Size Distribution and It's Effect on the Permeability of Unconsolidated Sands", Water Resources Research, Vol. 2, No. 4, 1966.

43. Martin, R., "On spatial dependence, bias and use of first spatial differences in Regression Analysis", Area, Vol. 6, 185-194, 1974.

44. Matsuo, M., Reliability in Embankment Design, Massachusetts Institute of Technology Publication R76-33, July 1978. 
45. McMillan, W.D., Theoretial Analysis of Groundwater Basin Operations, Water Resources Center Contribution 114, University of California, Berkley, CA, 1966.

46. Miller, Irwin and Freund, John E., Probability and Statistics for Engineers, Prentice-Hall, Inc., New Jersey, 1977.

47. Muskat, M., The Flow of Homogeneous Fluids Through Porous Media, McGraw Hill, 1946.

48. Naval Facilities Engineering Command, Soil Mechanics-Design Manual 7.1. May, 1982 .

49. Newland, D.E., An Introduction to Random Vibrations and Spectral Analysis, Longman Group Ltd., London, 1975.

50. Nielson, Biggar and Erh, "Spatial Variability of Field Measured Soil Water Properties", Hilgardia, Vo1. 42, No. 7, 1973.

51. Ogata, A., Theory of Dispersion in a Granular Medium, U.S. Geological Survey, Prof. Paper 411-I, 1970.

52. Peck, R.B., Hanson, W.E. and T.H. Thornburn, Foundation Engineering, New York: John Wiley and Sons, 1953.

53. Pettyjohn, F.J., Sedimentary Rocks, Harper and Brothers, New York, 1957. 
54. Powers, J.P., Construction Dewatering: A Guide To Theory And Practice, John Wiley and Sons, 1981.

55. Prickett, T.A. "Modeling Techniques for Groundwater Evaluation" In Advances In Hydroscience Vol. 10, Academic Press, New York, 1975 .

56. Prickett, T.A. and Lonnquist, C.G., Selected Digital Computer Techniques for Groundwater Resource Evaluation by I1linois State Water Survey, Bulletin 55, 1971.

57. Prickett, T.A.; Naymik, T.G., and Lonnquist, C.G., A Random Walk Solute Transport Model For Selected Groundwater Quality Evaluations by Illinois State Water Survey Bulletin 65, 1981 .

58. Reiter, P.F., "A Computer Study of the Correlation Between Aquifier Hydraulic and Aquifier Electric Properties," A thesis submitted in partial fulfillment of the requirements for the degree of Master of Science in Civil and Environmental Engineering, University of Rhode Island, 1981.

59. Rushton, K.R. and Redshaw, S.C., Seepage and Groundwater Flow, John Wiley \& Sons, New York, 539p, 1979.

60. Sauty, J.P., "Computer Simulation of Pollution Front Movement", Conference Presentation in France, 1976. 
61. Smith, L., "A stochastic analysis of steady-state groundwater flow in a bounded domain", dissertation presented to the University of British Columbia, Vancouver, Canada in 1978 in partial fulfillment of the requirements for the degree of Doctor of Philosophy.

62. Smith, L., "Spatial Variability of Flow Parameters in a Stratified Sand," Mathematical Geology, Vol. 13, No. 1, 1981.

63. Smith, I. and R.A. Freeze, "Stochastic Analysis of Steady State Groundwater Flow in a Bounded Domain, 1, One-dimensional Simulations", Water Resources Research, Vo1. 15, 521-528, 1979 a.

64. Smith L., and R.A. Freeze, "Stochastic Analysis of Steady State Groundwater Flow in a Bounded Domain, 2, Two-Dimensional Simulations", Water Resources Research, Vol. 15, 1543-1559, 1979 b.

65. Smith, L. and F. Schwartz, "Mass Transport I.A Stochastic Analysis of Macroscopic Dispersion", Water Resources Research, Vo1. 16, No. 2, April 1980.

66. Sta1lman, R.W., "Aquifer Test Design, Observation and Data Analysis", Techniques of Water Resources Investigation of the U.S. Geological Survey, Chap. B1, Government Printing Office, Washington, D.C., 1971. 
67. Tabba, M.M. and Yong, R.M., "Mapping ond Predicting Soil Properties: Theory", Journal of the Engineering Mechanics Division, ASCE, Vo1. 107, No. EM5, Proc. Paper 16534, Oct., 1981, p. 773-793.

68. Tabba, M.M. and Yong, R.N. "Mapping and Predicting Soil Properties: Applications," Journal of the Engineering Mechanics Division, ASCE, Vol. 107, No. EM5, Proc. Paper 16533, Oct., 1981, pp. 795-811.

69. Todd, D.K. Groundwater Hydrology. John Wiley \& Sons, New York, 1980.

70. Trescott, P.C., Pinder, G.F. and Larson, S.P., Techniques of Water-Resources Investigations of the U.S.G.S., Chapt. C1. "Finite-Difference Model for Aquifer Simulation in TwoDimensions with Results of Numerical Experiments", U.S. Gov. Printing Office, 1976.

71. Van Marke, Eric H., "Probabilistic Modeling of Soil Profiles", Journal of the Geotechnical Division, ASCE, Vol. 103, GT 11, Nov. 1977.

72. Van Marke, Eric H., "Reliability of Earth Slopes", Journal of the Geotechnical Division, ASCE, Vol. 103, GT11, Nov., 1977.

73. Van Marke, Eric H., "Probabilistic Characterization of Soil Profiles" in Proceedings of Specialty Workshop, Northwestern University, Evanston, Illinois, June, 1978. 
74. Walpole, Ronald E., and Myers, Raymond H., Probability and Statistics for Engineers and Scientists, Macmillan Publishing Co., New York, 1978.

75. Wang, H.F. and M.P. Anderson, Introduction to Groundwater Modeling, Finite Difference and Finite Element Methods, W.H. Freeman and Co., San Francisco, 1982.

76. Warren, J.E. and M.S. Price, "Flow in Homogeneous Porous Media", Society of Petroleum Engineers, Sept. 1961.

77. Warren, J.E. and F.F. Skiba, "Macroscropic Dispersion," Soc. Petrol. Eng. J. 215-230, Sept., 1964.

78. Whitten, E.H.T. Compositional Trends in a granite modal variation and ghost-stratigraphy in part of the Donegal Granite", Eire: Jour. Geophys. Research, Vo1. 64, P. 835-848, 1959.

79. Whittle, P., "Stochastic processes in several dimensions", Bull. Int. Statist. Inst., Vol. 40, 974-994, 1963.

80. Willardson, L.S. and Hurst, R.L., "Sample Size Estimates in Permeability Studies", Journal Irrig. and Drain. Div., ASCE, Vol. $91,1965$.

81. Wu, Tien H., Vyas, Shyam K. and Chang, N.Y., "Probabilistic Analysis of Seepage" Journal of the Soil Mechanics and Foundations Division, ASCE, Vol. 99, SM4, 1973. 


\section{APPENDIX D - COMPUTER PROGRAMS}

\section{INVERSE}

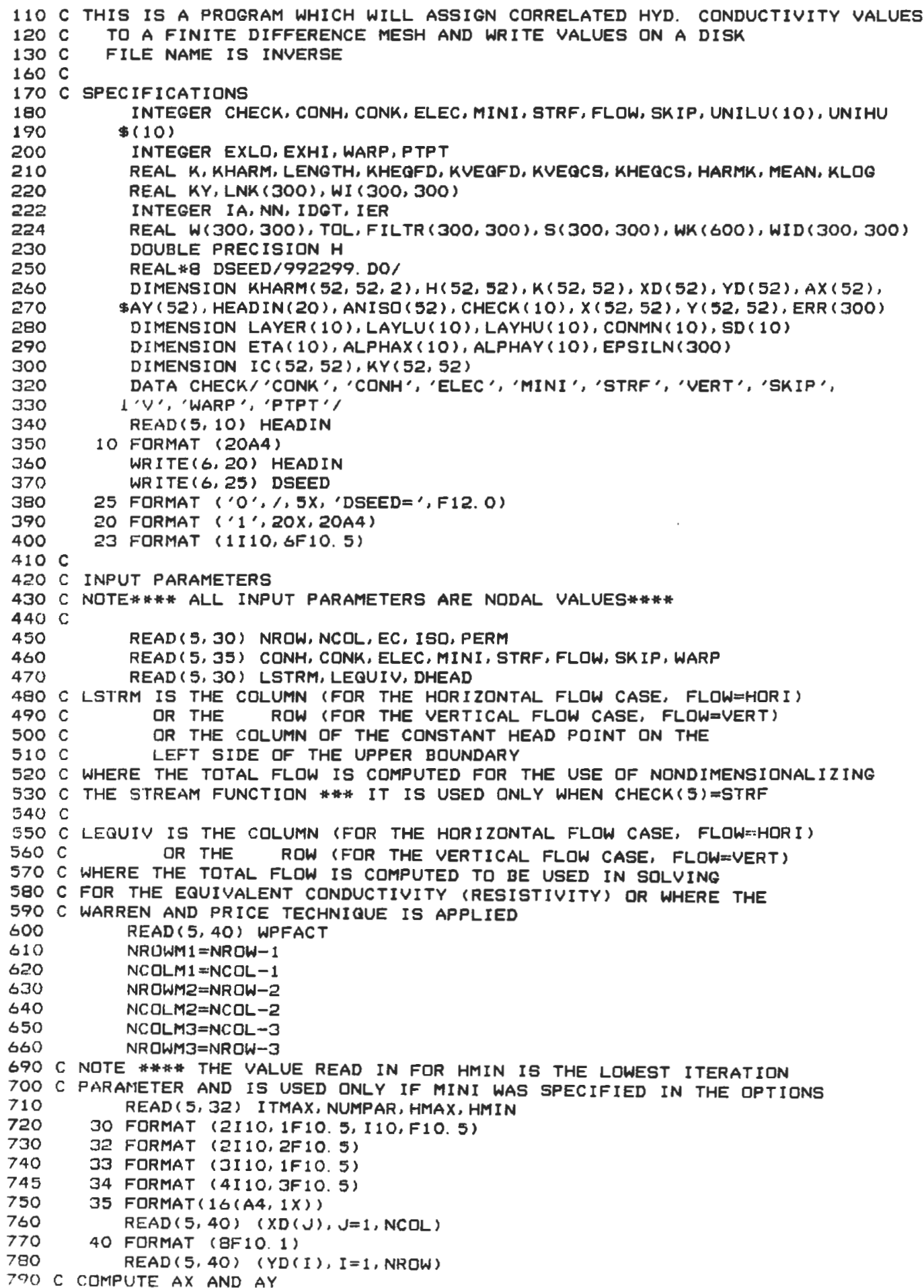




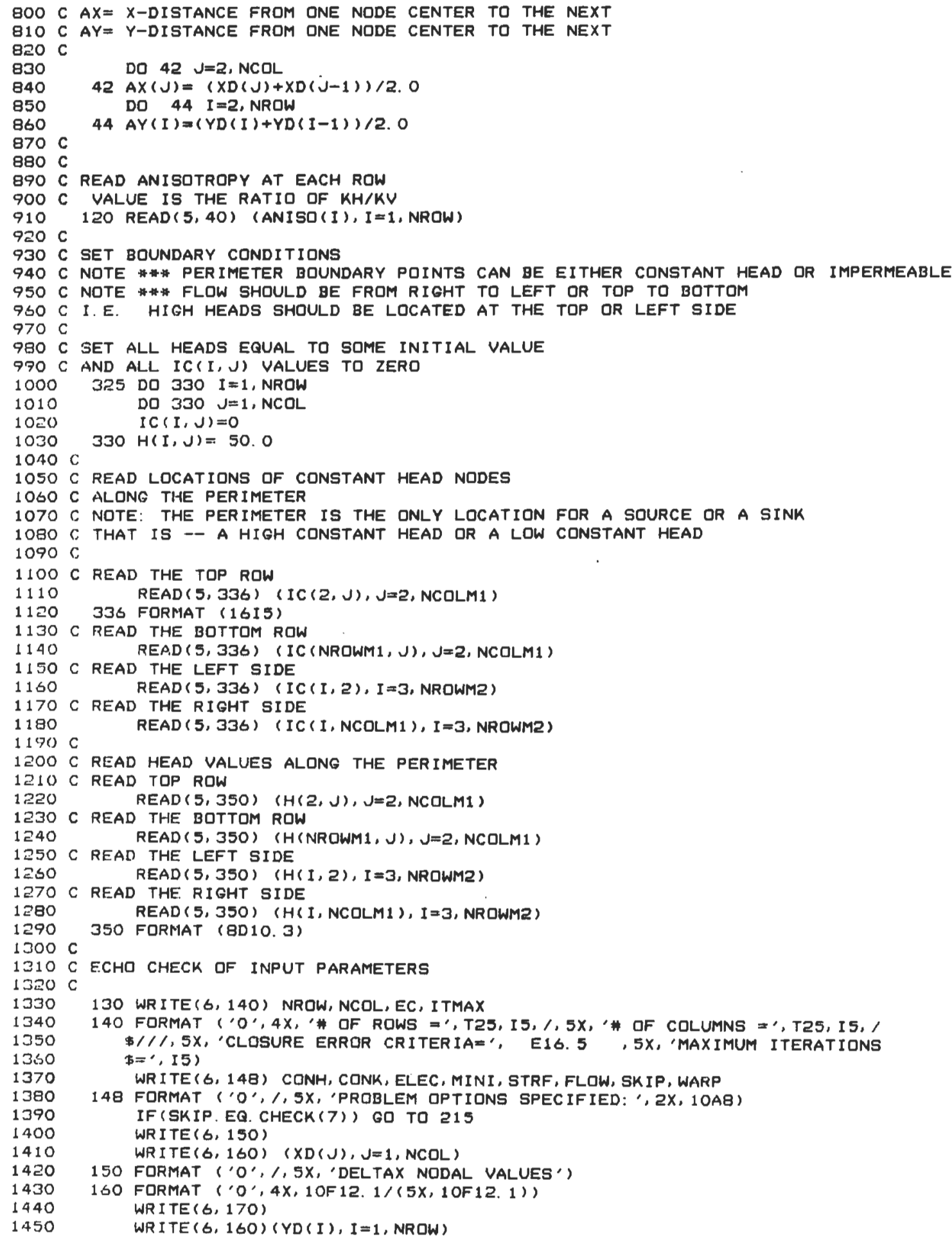




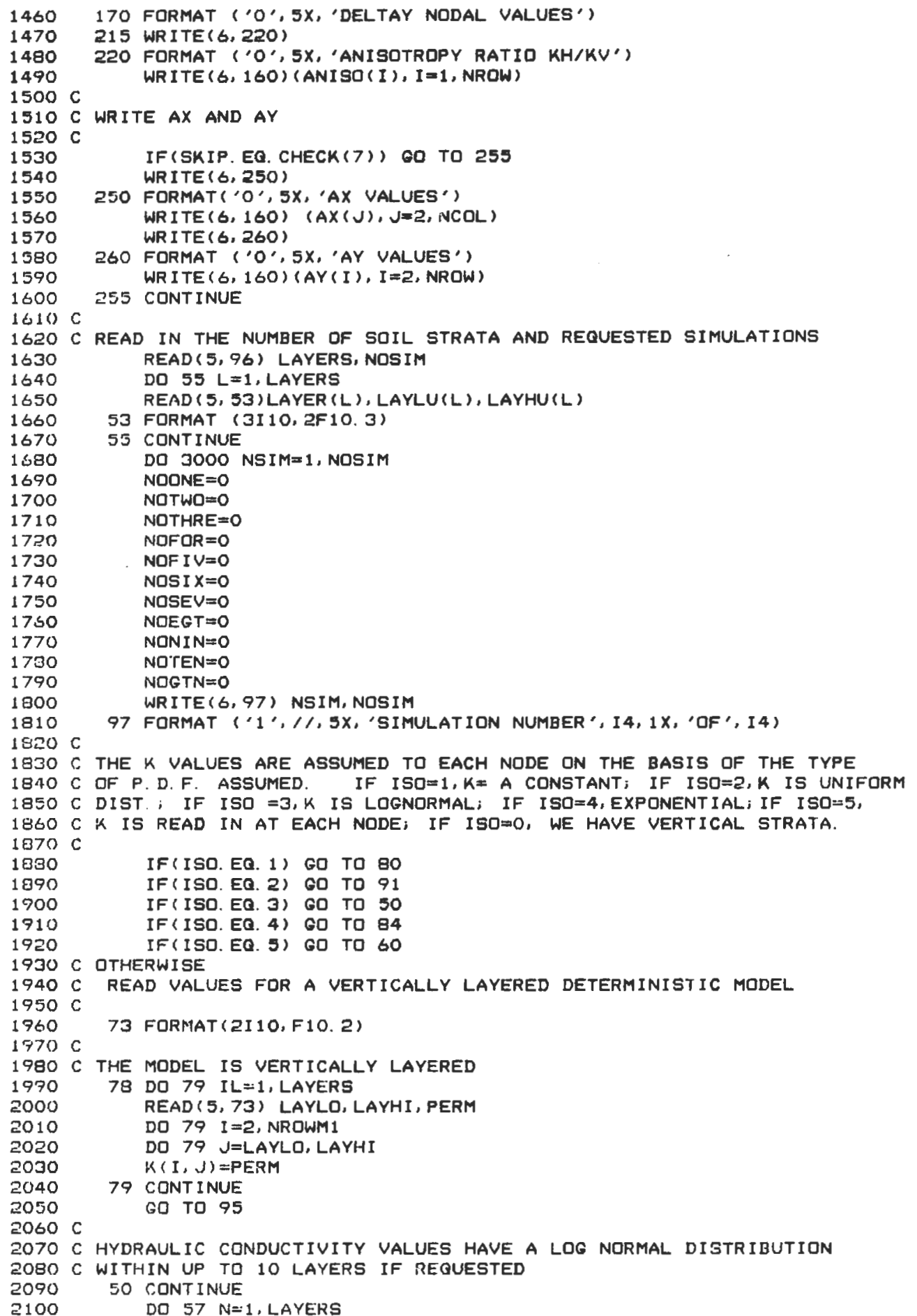




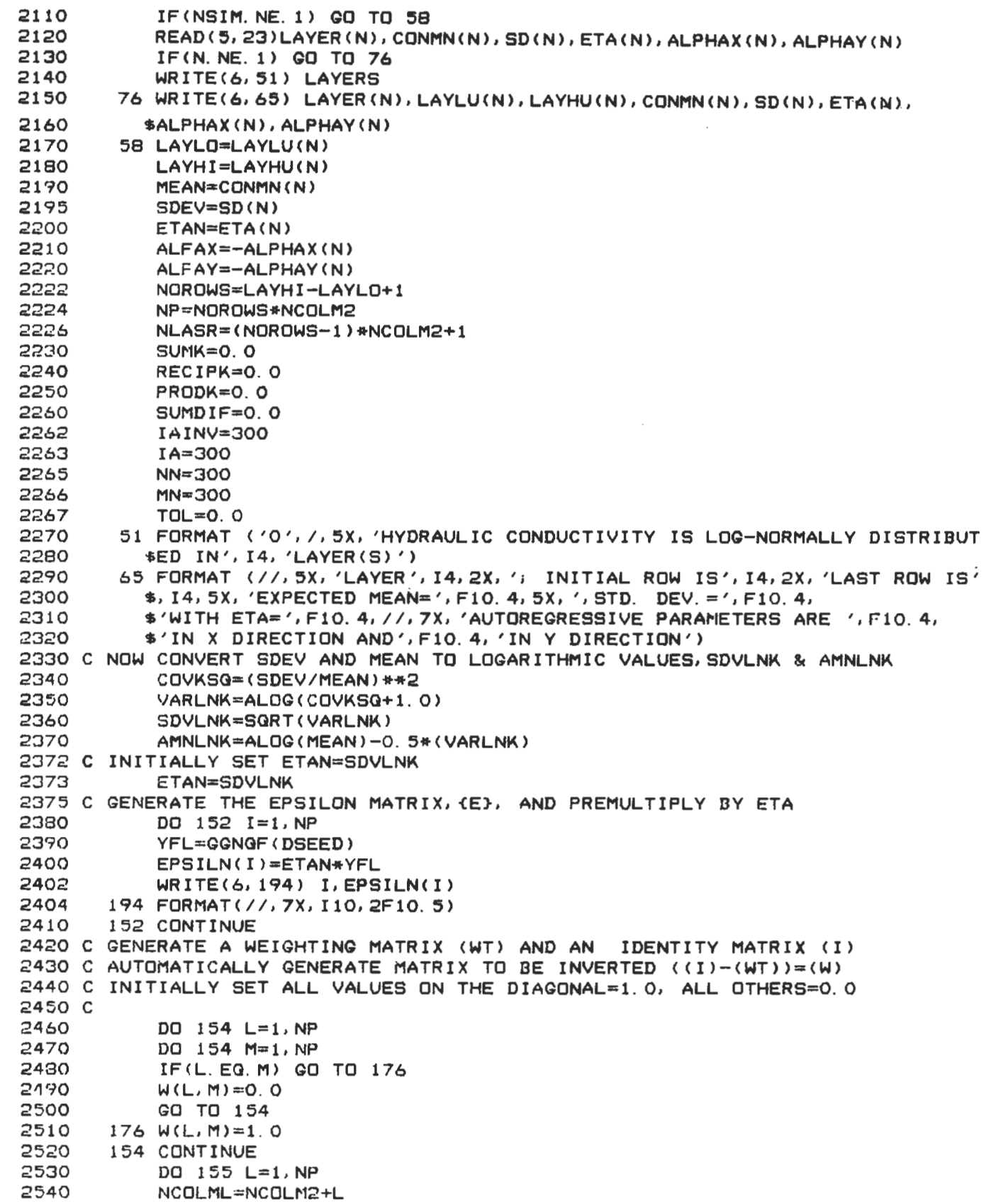




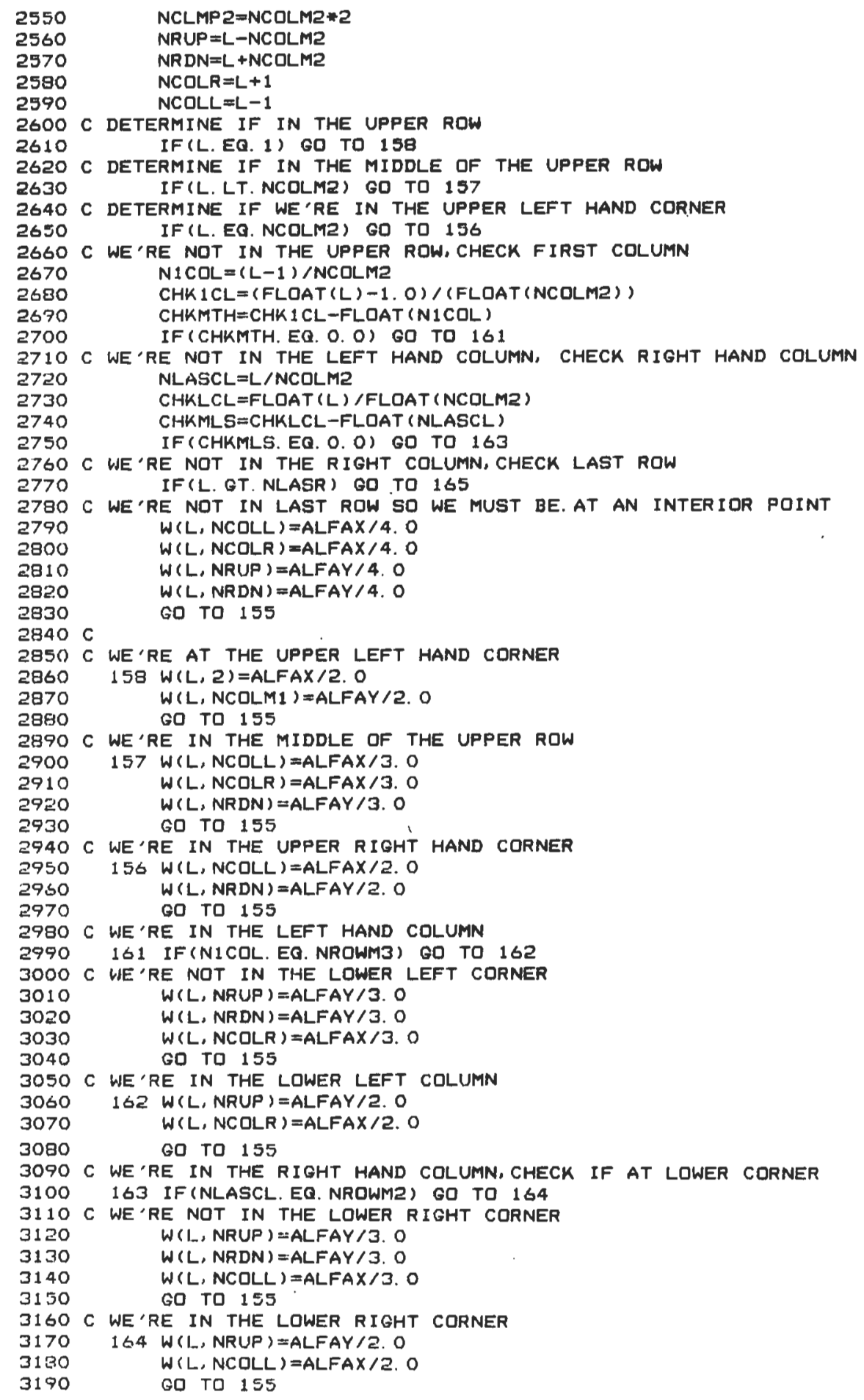




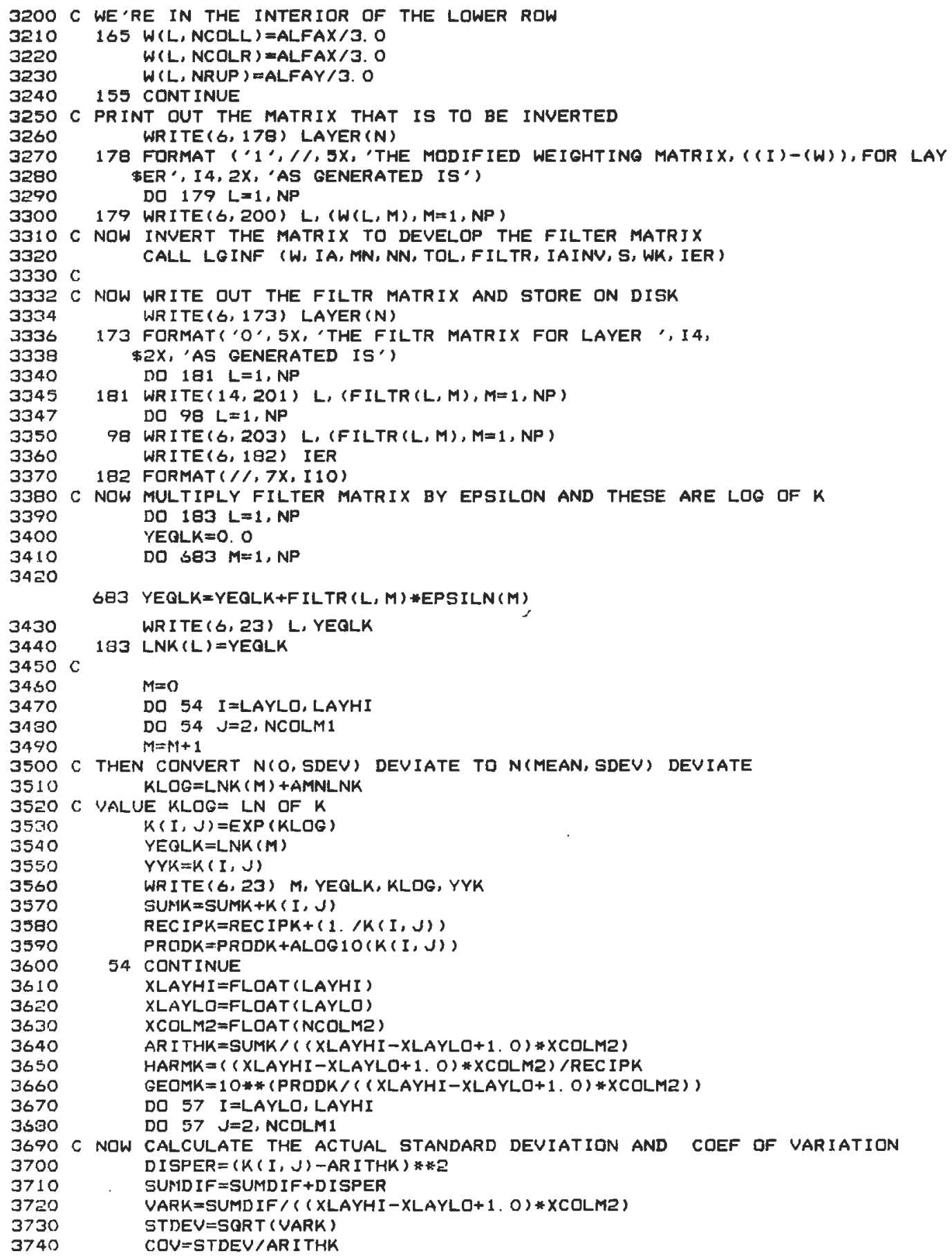




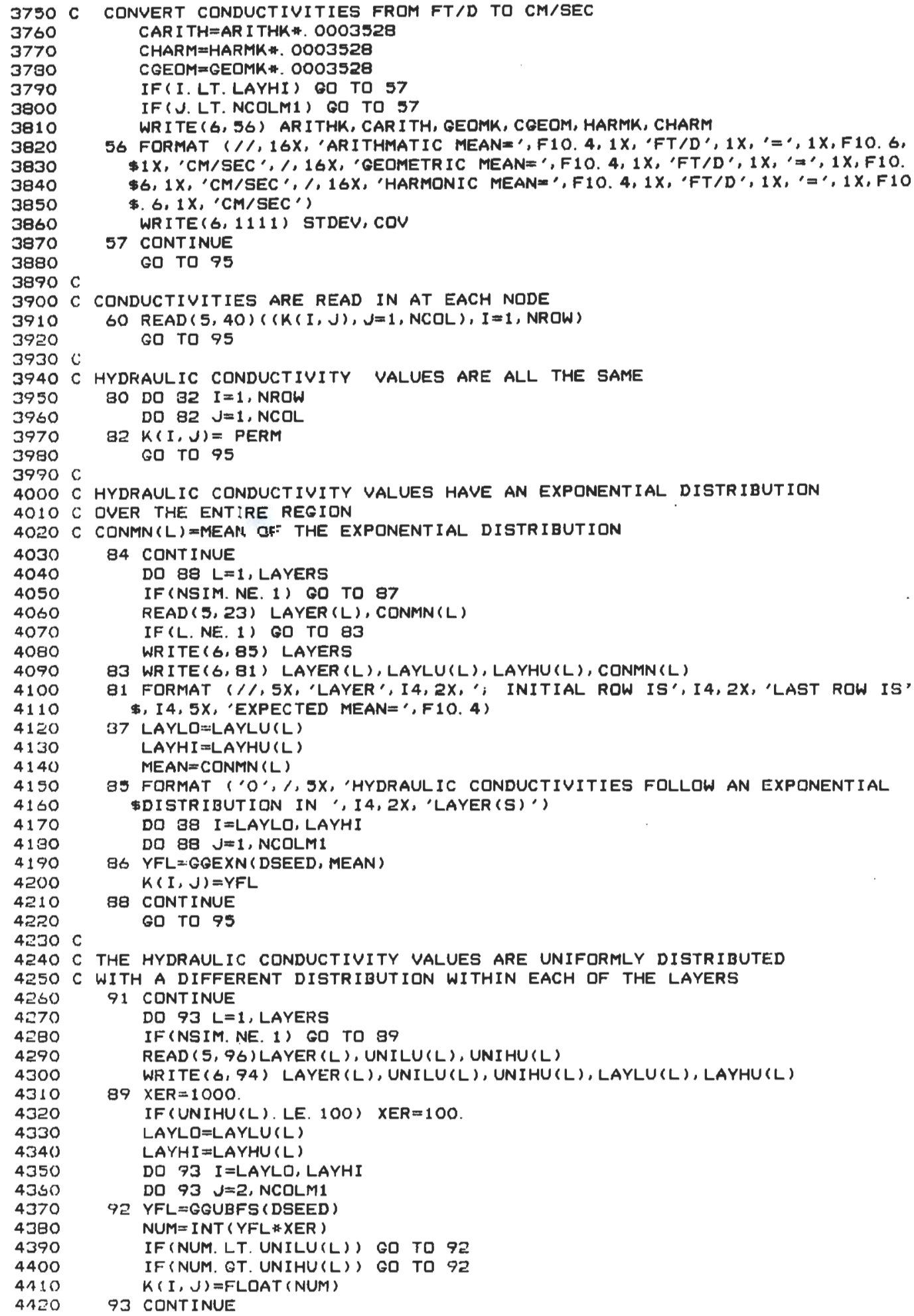




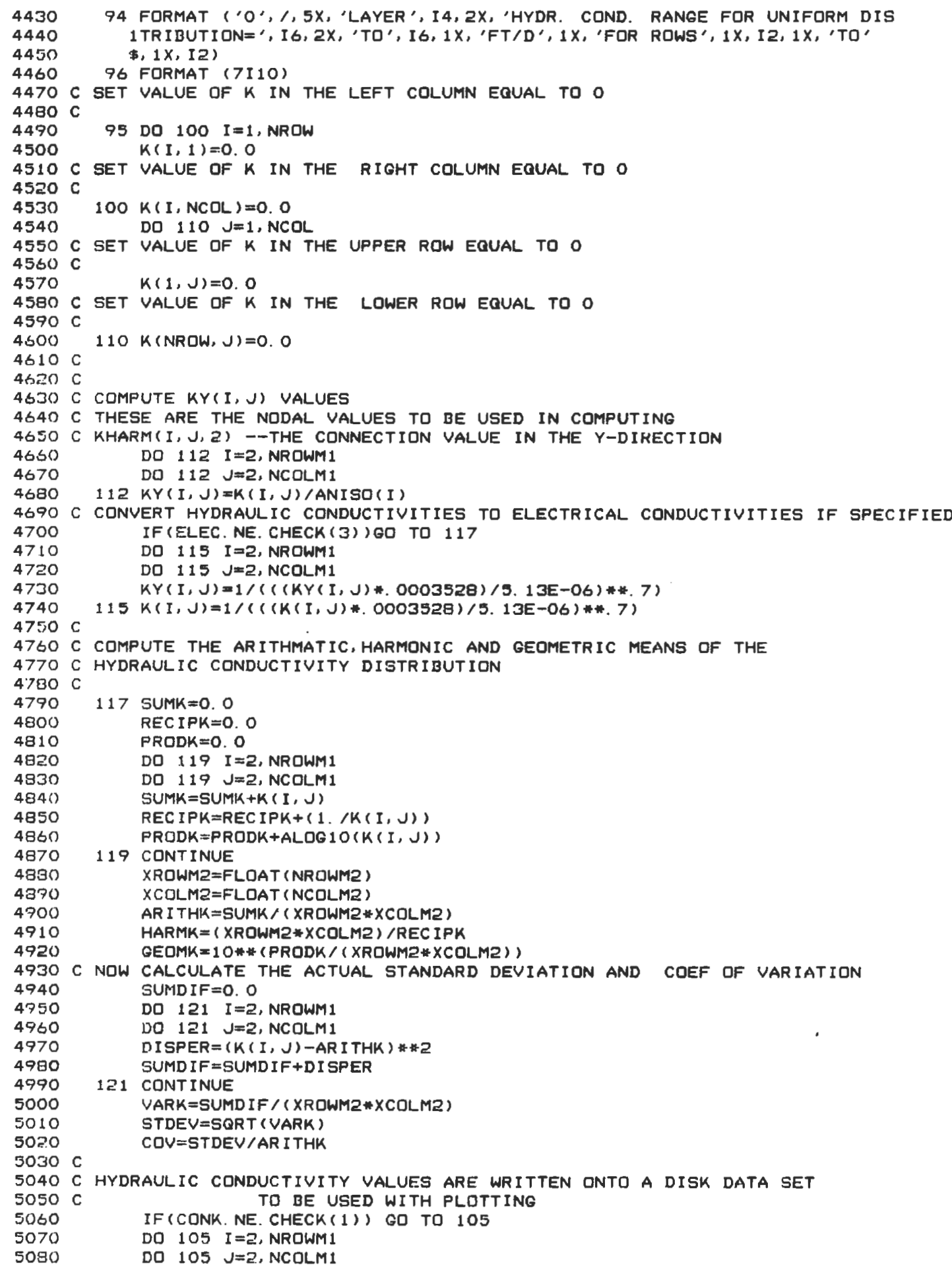




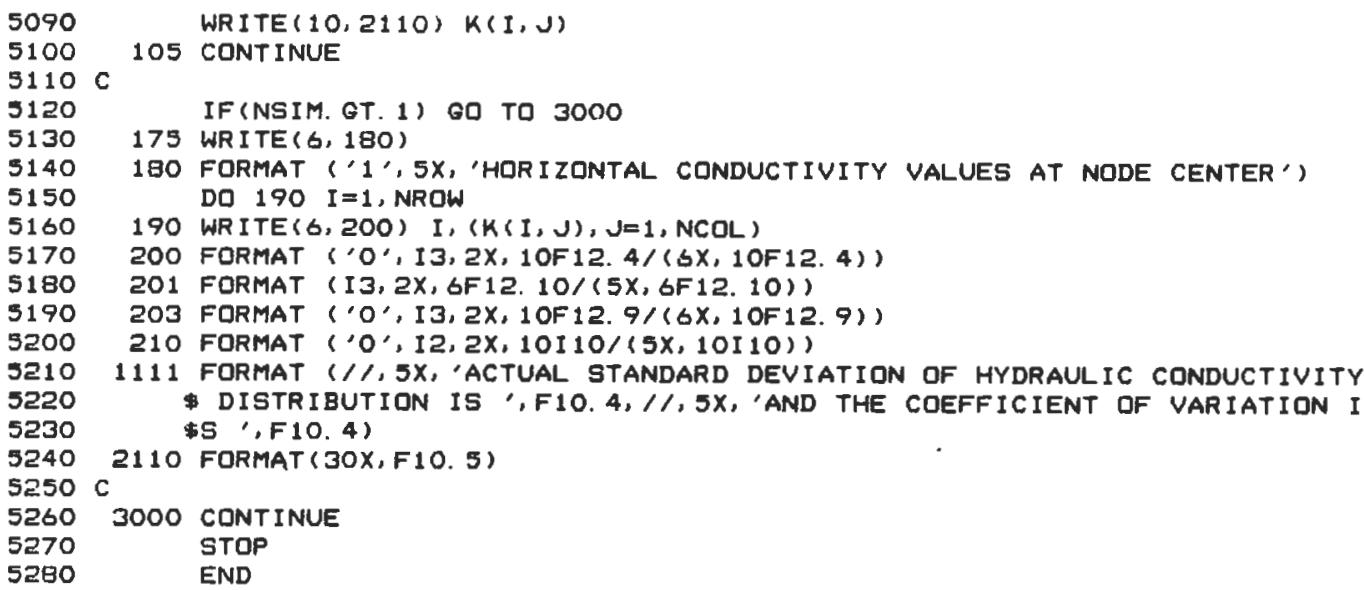

\section{STKBLKNP}

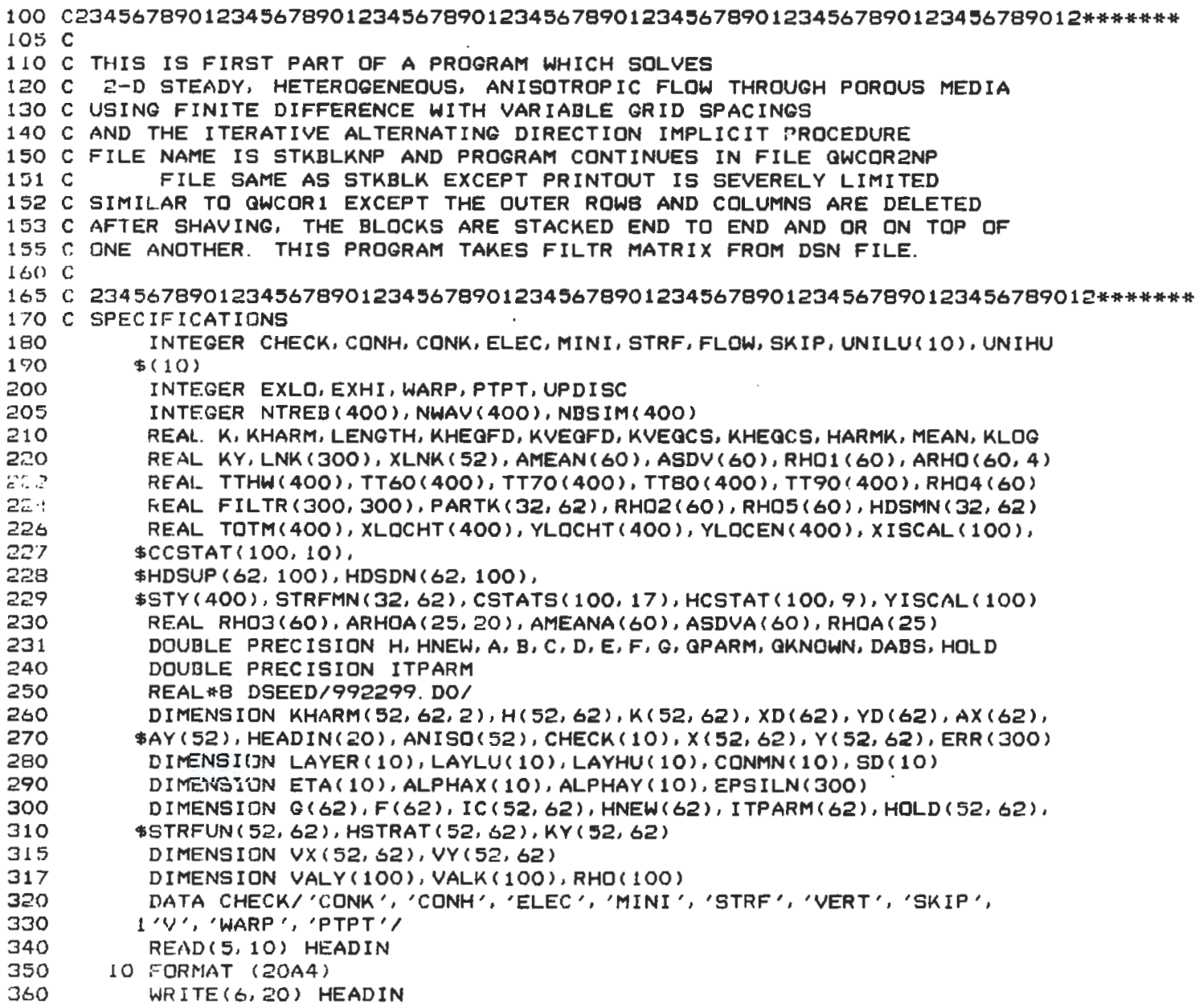




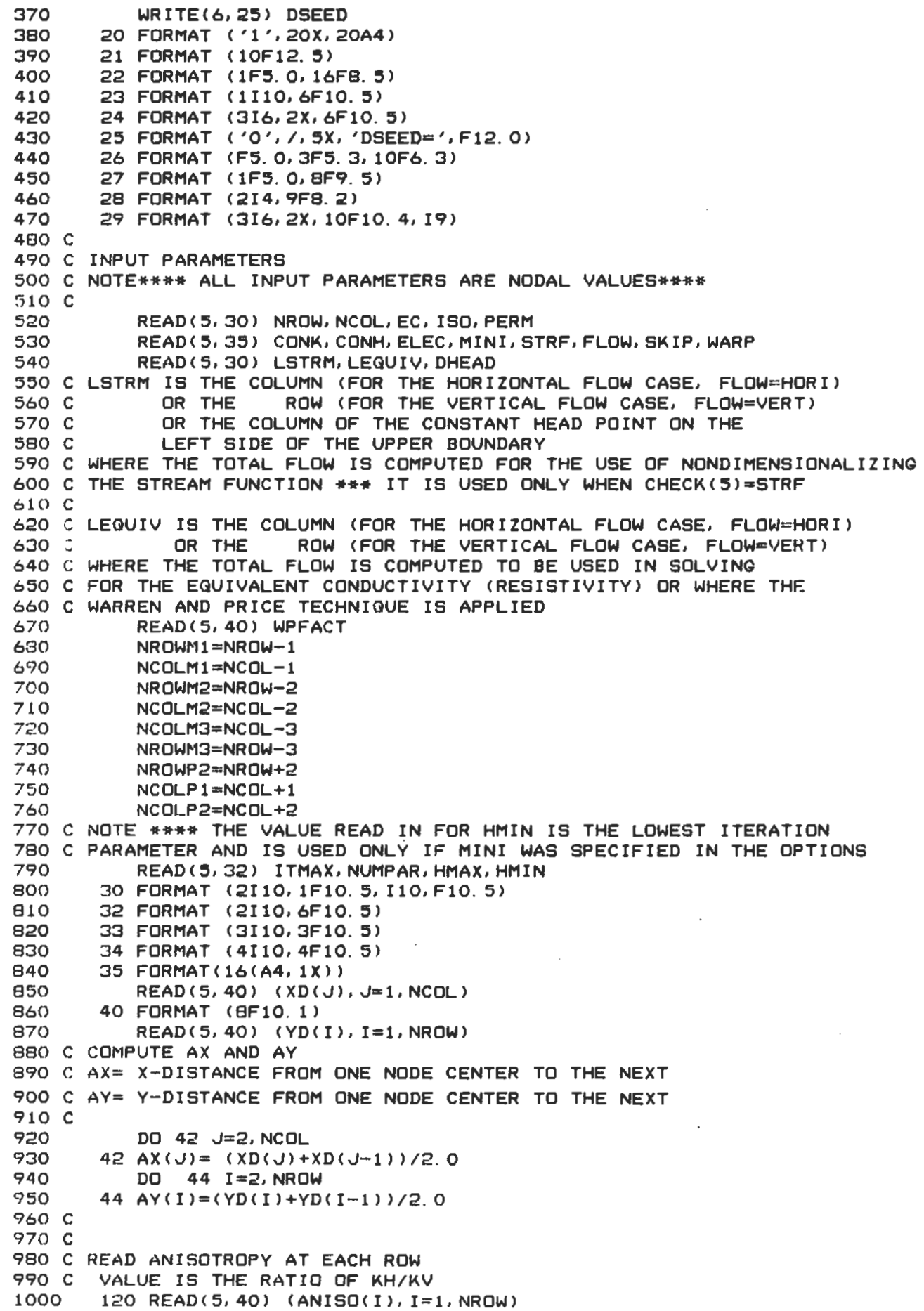




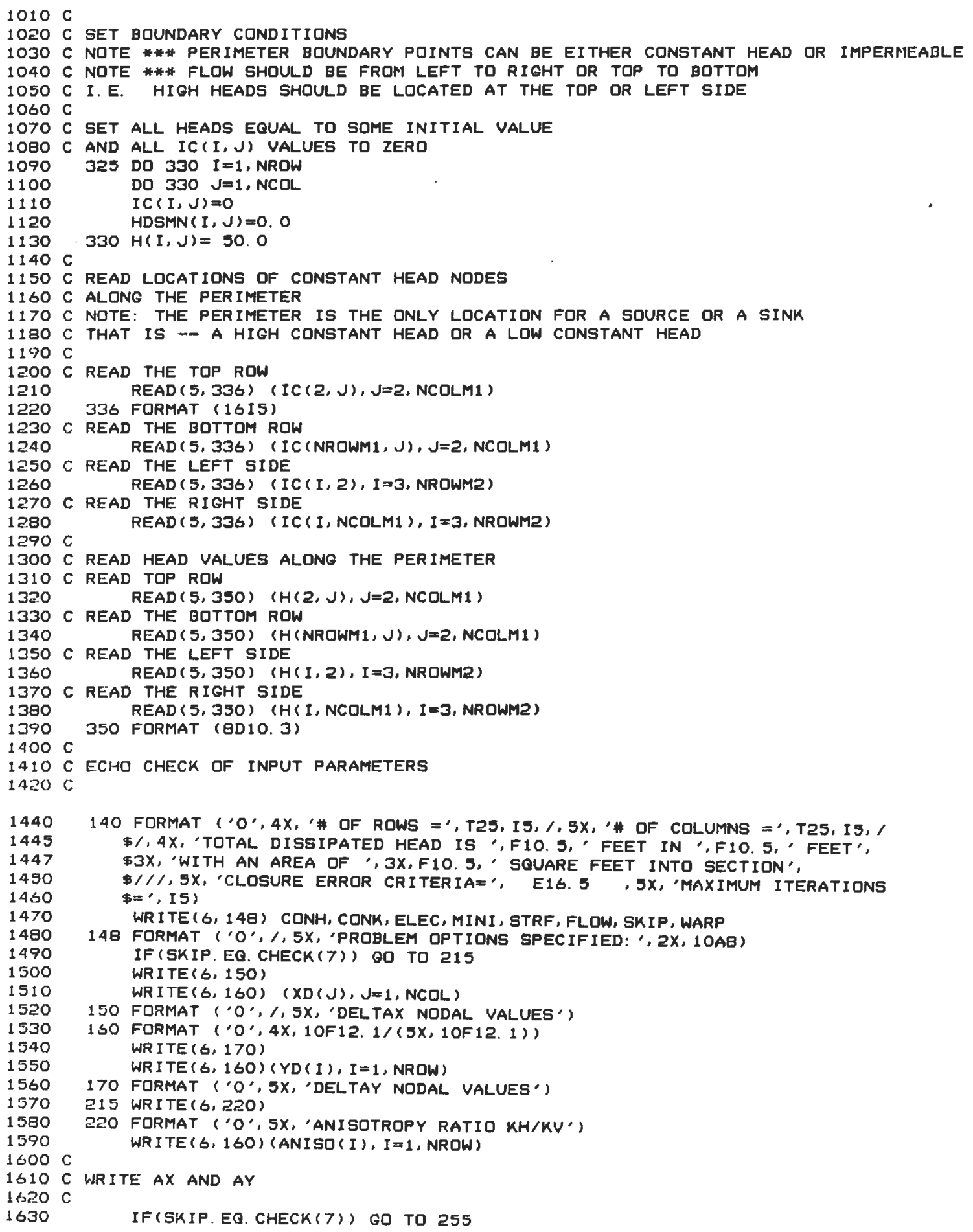




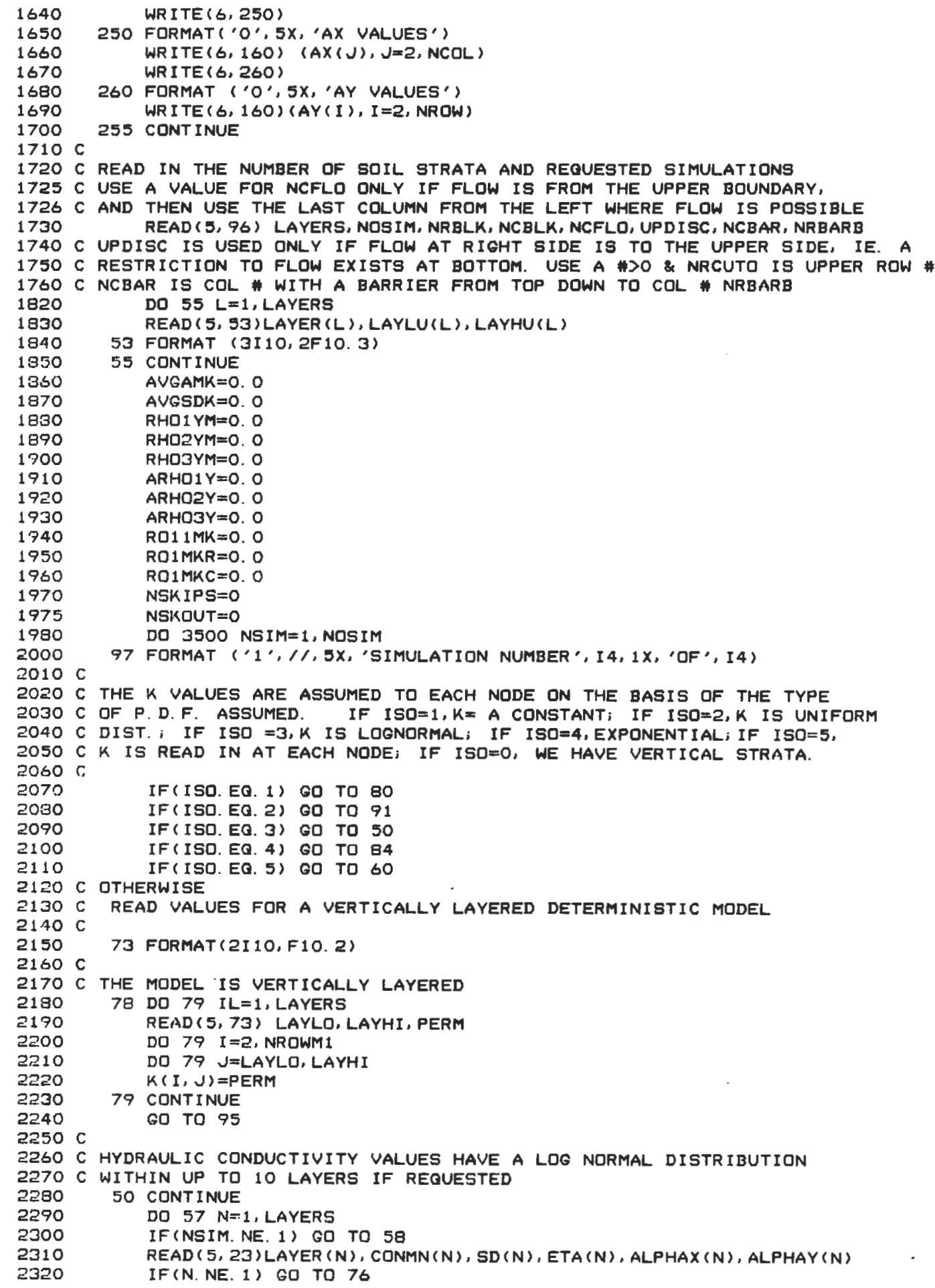




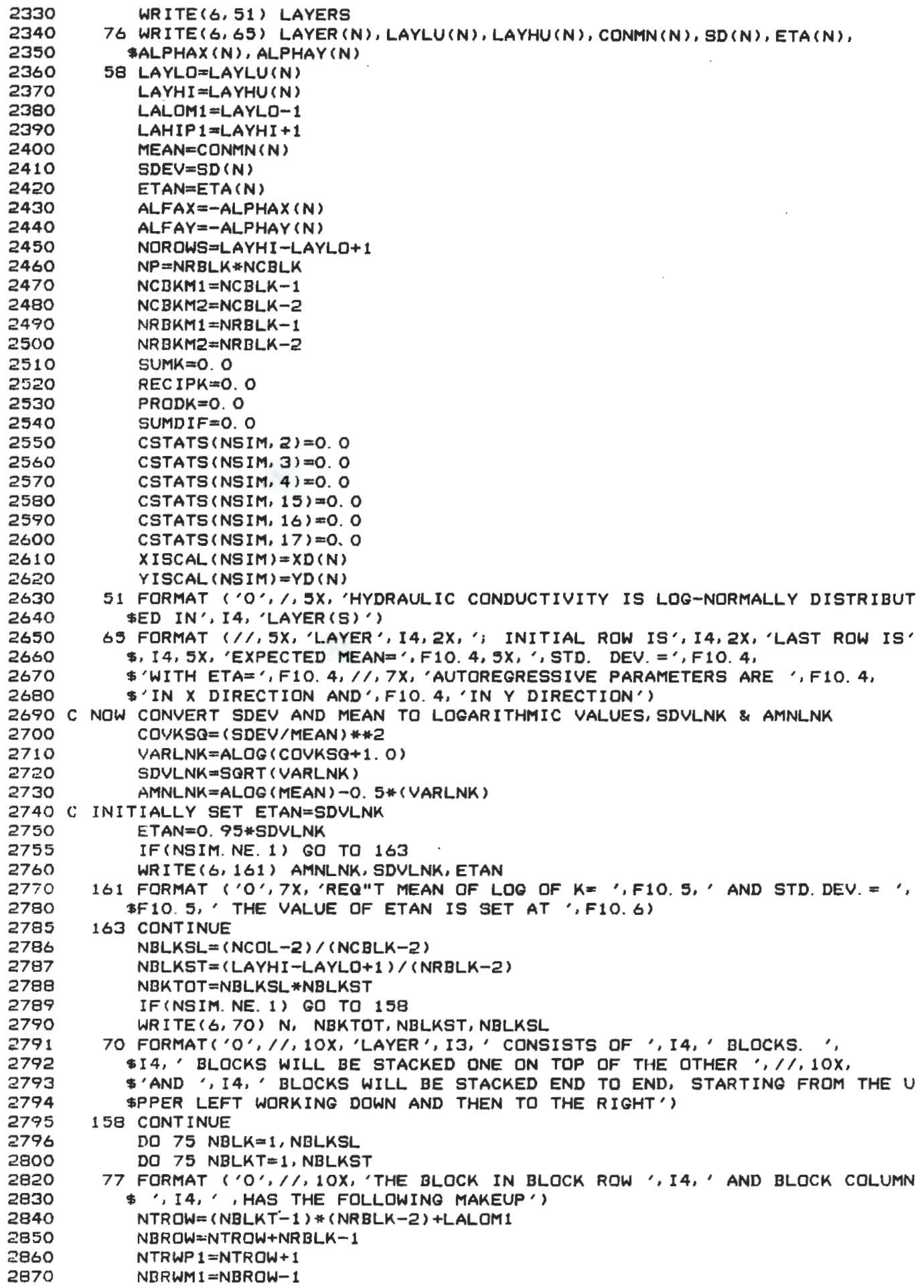




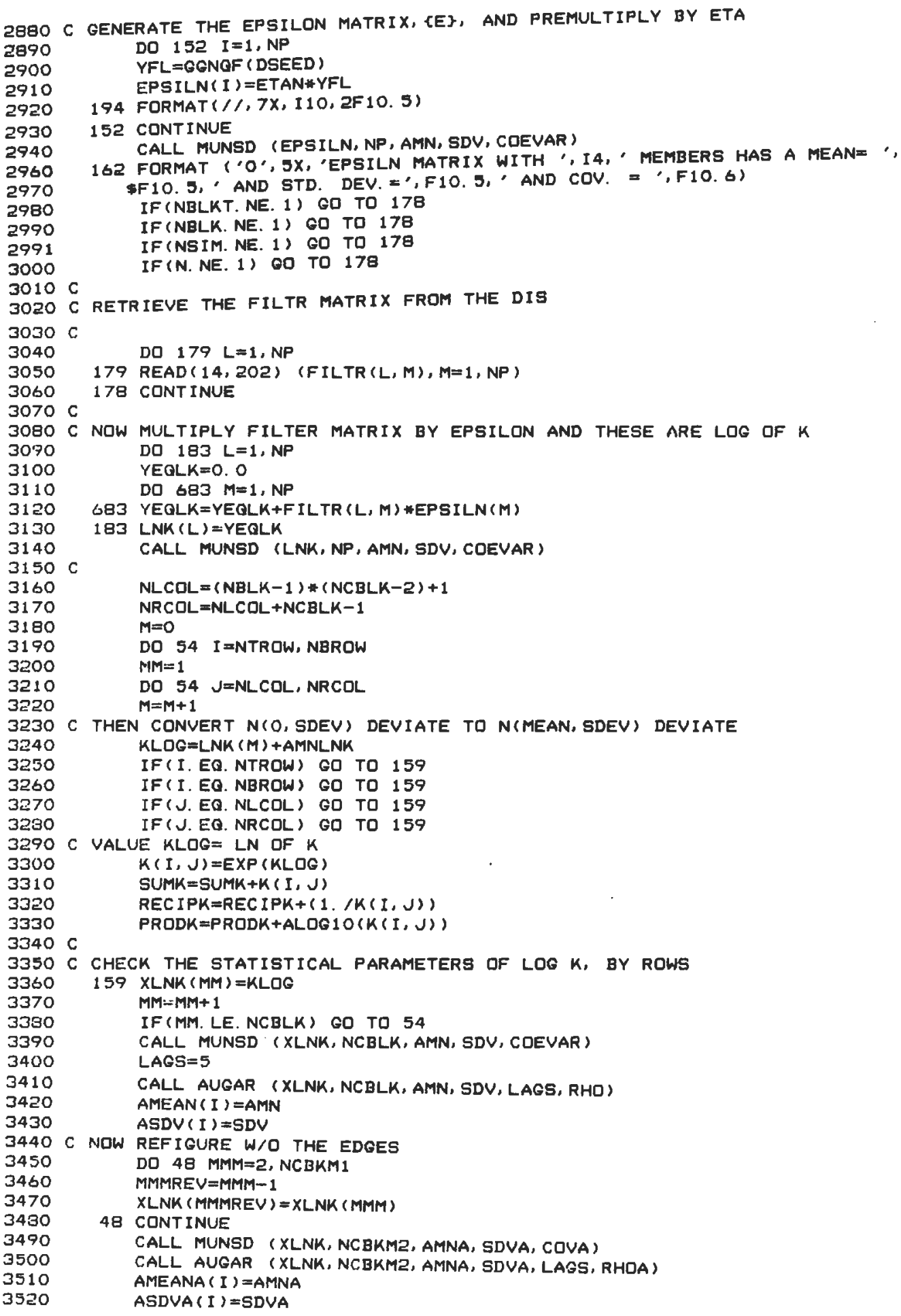


DO 54 IN=1, LAGS

$A R H O(I, I N)=R H O(I N)$

$A R H Q A(I, I N)=R H O A(I N)$

54 CONTINUE

CORRELATION COEFFICIENTS FOR ALL ROWS OF THIS SEGMENT HAVE BEEN CALCULATED DO 52 I $=$ NTROW, NBROW

IREV $=I-N T R O W+1$

RHO $1(\operatorname{IREV})=\operatorname{ARHO}(1,1)$

RHO2 (IREV) =ARHO (I, 2$)$

RHO3 (IREV) $=A R H O(I, 3)$

52 CONTINUE

64 FDRMAT' 0 ', 10X, 'MEAN, STD. DEV. AND AUTDCORRELATIDN COEFFICIENTS FO

$\$ R$ EACH ROW AND LAGS FROM 1 TO 4 ', $1,7 X$, 'ROW', $4 X$, 'MEAN', $5 X$,

क'STD. DEV. ', 2X, 'RHO(1)', 4X, 'RHO(2)', 4X,'RHO(3)', 4X,'RHO(4)',

CALL MUNSD (RHOI, NRBLK, AMN, SDV, COEVAR)

RHOI YM $=R H D 1 Y M+A M N /$ (FLOAT (NDSIM))

CSTATS (NSIM, 1) =FLDAT (NSIM)

CSTATS (NSIM, 2) $=$ CSTATS (NSIM, 2) +AMN $/($ FLOAT (NBKTOT) )

165 FORMAT ('O', 5X, 'LAG I CORR. COEF. FOR', I3,' ROWS, HAS A MEAN=', \$F 10. 5,' AND STD. DEV. =',F10. 5,' AND COV. = ',F10. 6)

CALL MUNSD (RHD2, NRBLK, AMN, SDV, COEVAR)

RHO2YM=RHO2YM+AMN/ (FLOAT (NDSIM))

CSTATS (NSIM, 3) =CSTATS (NSIM, 3)+AMN/(FLOAT (NBKTQT) )

168 FORMAT ('O', $5 X$, 'LAG 2 CORR. COEF. FOR', I3,' ROWS, HAS A MEAN=', \$F10. 5,' AND STD. DEV. =',F10. 5,' AND COV. = ',F10.6)

CALL MUNSD (RHO3, NRBLK, AMN, SDV, CQEVAR)

RHO3YM $=$ RHO3YM+AMN/(FLOAT (NOSIM))

CSTATS (NSIM, 4) =CSTATS (NSIM, 4) +AMN/(FLDAT (NBKTQT))

172 FORMAT ('O', $5 X$, 'LAG 3 CORR, COEF. FOR', I3,' ROWS, HAS A MEAN $=$ ', $\$ F 10.5$, AND STD. DEV. $=\prime, F 10.9$, AND CDV. = ', F10.6)

139 FORMAT $(' 0 \circ, 5 X$, 'LAG 1 CORR. COEF. FOR', I3,' COLMNS, HAS A MEAN=', \$F 10. 5,' AND STD. DEV. =',F10.5,' AND COV. = 'F10. 6)

171 FORMAT $(' 0$ ', 5X, 'LAG 2 CORR. COEF. FDR', I3,' COLMNS, has a Mean=', \$F 10. 5, AND STD. DEV. =',F10. 5, 'AND COV. = 'F10.6)

173 FORMAT ( $O \circ, 5 \mathrm{X}$. 'LAg 3 CORR. COEF. FOR', I3,' COLMNS, has a Mean=', \$F 10. 5, AND STD. DEV. =',F10. 5, AND COV. = 'F10.6)

69 FORMAT' $O$ ', 1OX, 'MEAN, STD. DEV. AND AUTOCORRELATION COEFFICIENTS FO \$R EACH COLUMN AND LAGS FROM 1 TO $4,1 / 1,7 X$, 'COLUMN', $4 X$, 'MEAN', $5 X$, '.'STD. DEV. ', 2X, 'RHO(1)', 4X, 'RHO(2)', 4X, 'RHD(3)', 4X, 'RHO(4)', C NOW CALCULATE MEAN CDRRELATION COEFFICIENTS AFTER DELETING OUTER NODES

71 FORMAT('O',7X, 'AFTER REMOUING THE OUTSIDE LAYERS OF THE CONDUCTIVI \$TY FIELD, WE ARE LEFT WITH A FIELD WITH THESE STATS') DO 153 I =NTRWP 1, NBRWM1 IREV = I -NTRWP $1+1$ RHO 1 ( IREV) $=A R H O A(I, 1)$ RHO2 (IREV) $=A R H O A(I, 2)$ RHO3 (IREV) $=A R H O A(I, 3)$

153 CDNTINUE DO 72 I=NTRWP 1, NBRWM1 IREV $=$ I -NTRWP $1+1$

72 CONTINUE NOROMZ=NOROWS-2

CALL MUNSD (RHO1, NRBKM2, AMN, SDV, COEVAR) ARHOIY=ARHOIY+AMN/ (FLOAT (NOSIM))

CSTATS (NSIM, 15) $=$ CSTATS (NSIM, 15) +AMN/ (FLOAT (NBKTOT) ) CALL MUNSD (RHOZ, NRBKM2, AMN, SDV, COEVAR) ARHOZY $=$ ARHOZY+AMN / (FLOAT (NOSIM)) CSTATS (NSIM, 16) $=$ CSTATS (NSIM, 16) +AMN/(FLOAT (NBKTOT)) CALL MUNSD (RHOJ, NREKM2, AMN, SDV, COEVAR) $A R H O 3 Y=A R H O 3 Y+A M N /($ FLDAT (NOSIM)) CSTATS (NSIM, 17) =CSTATS (NSIM, 17) +AMN/ (FLOAT (NBKTOT))

75 CONTINUE 


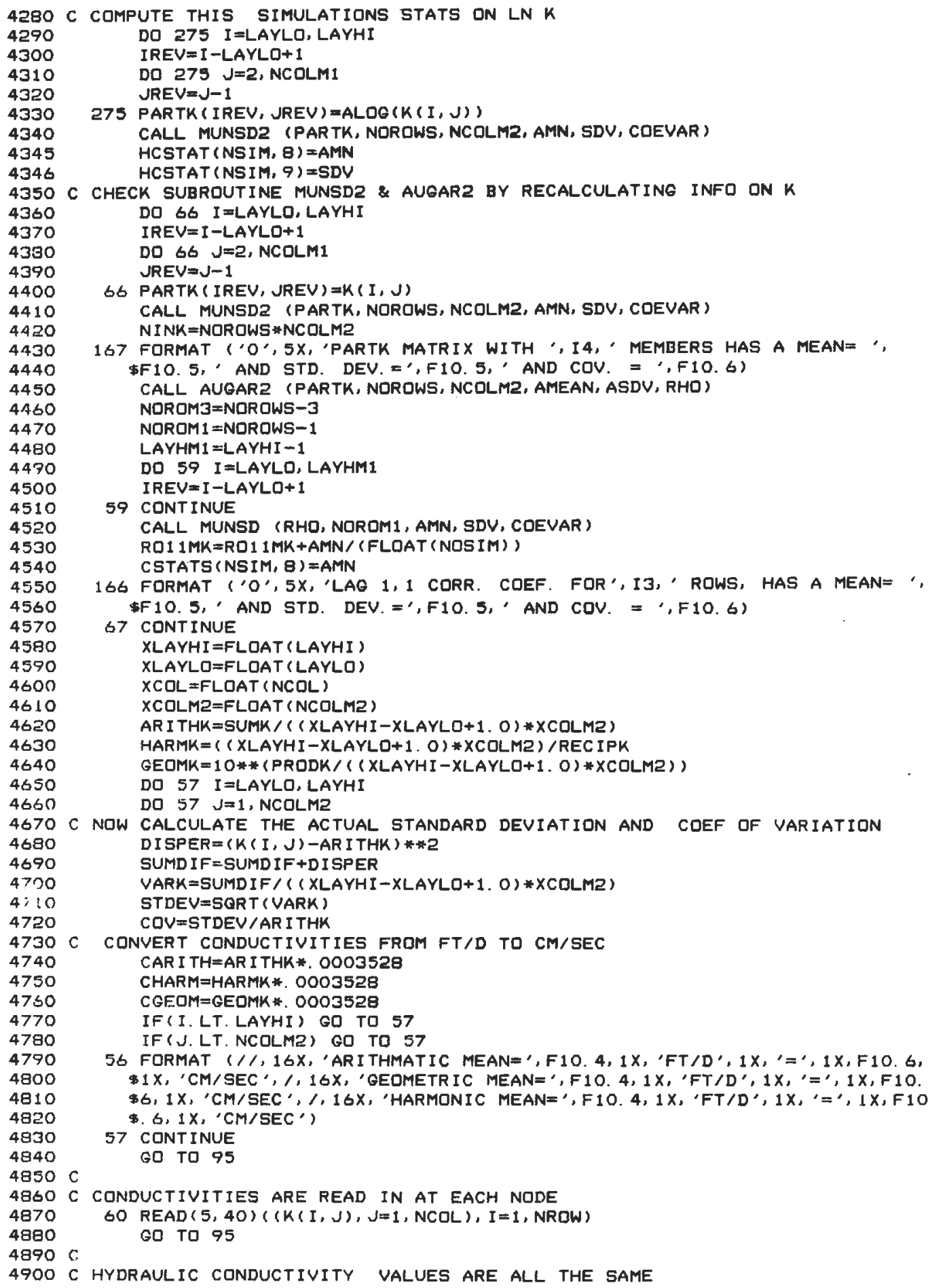




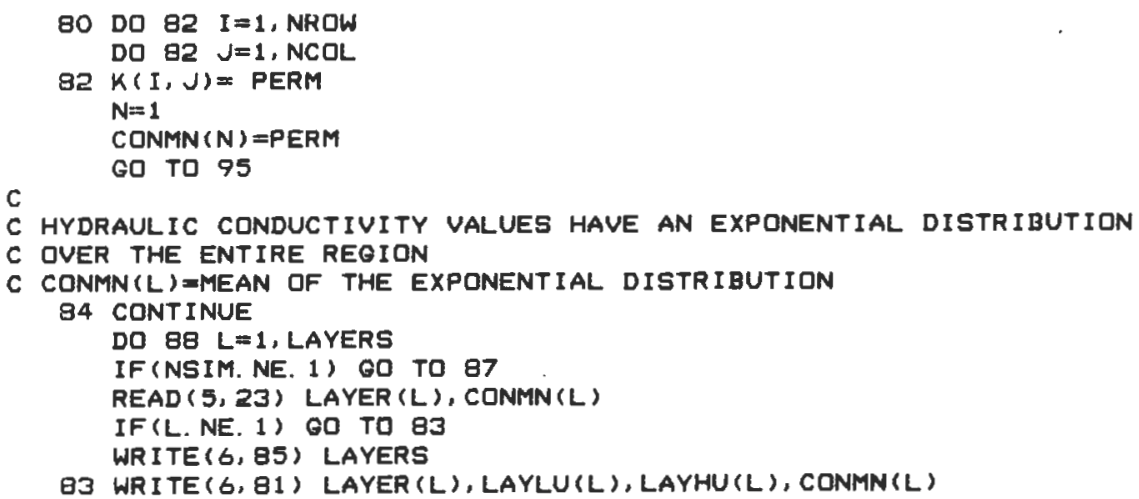

5570

5580
$5590 \mathrm{C}$ 


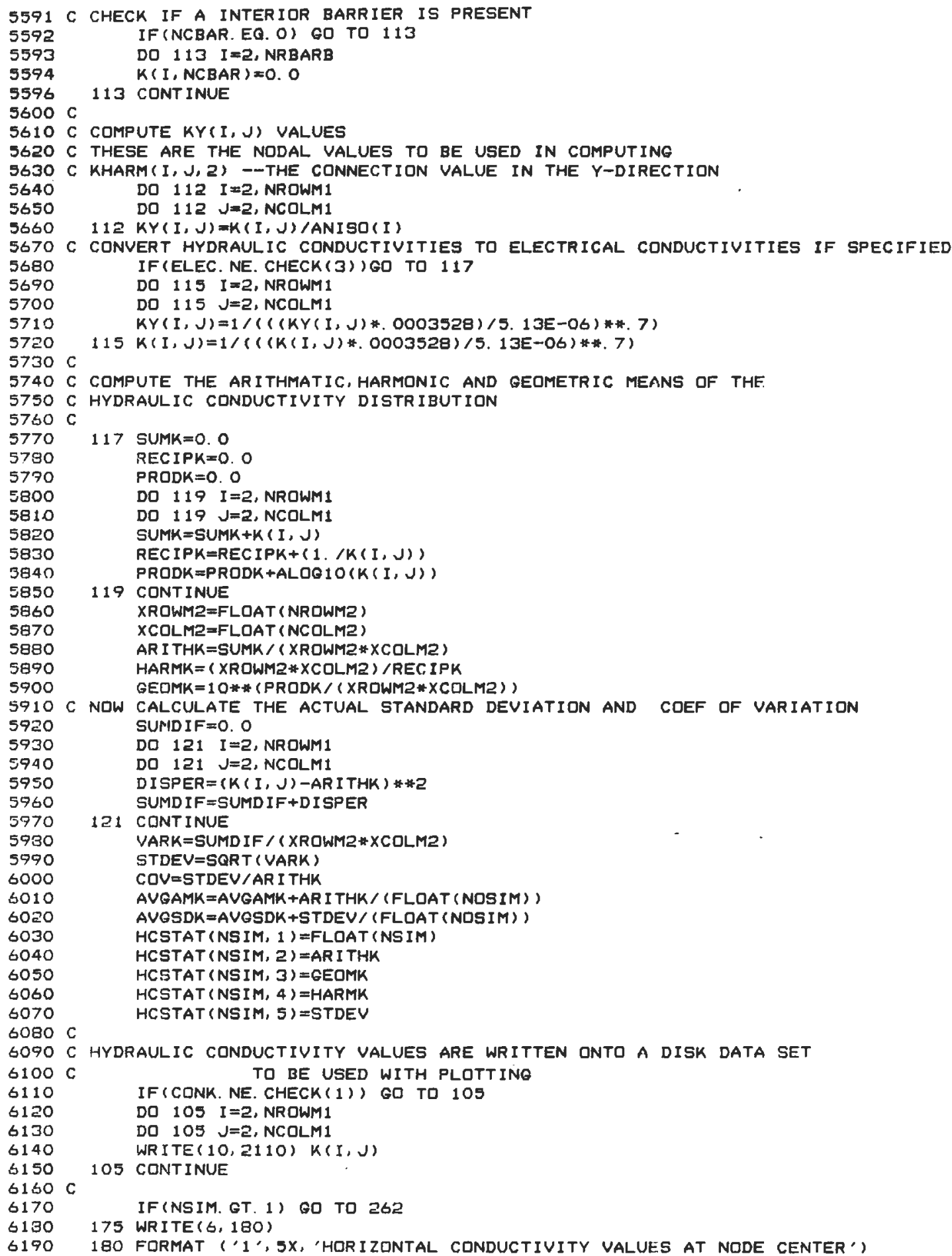




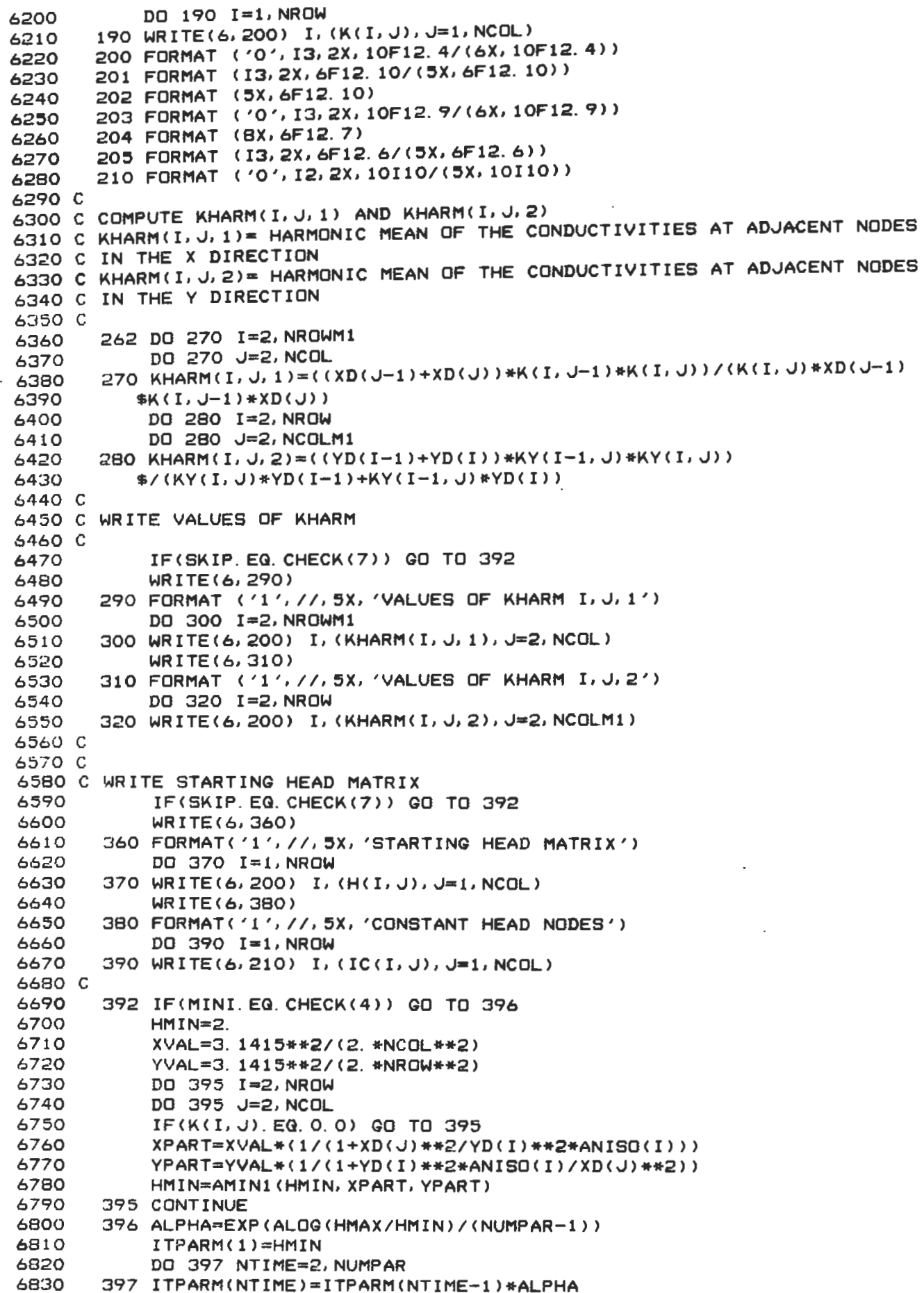




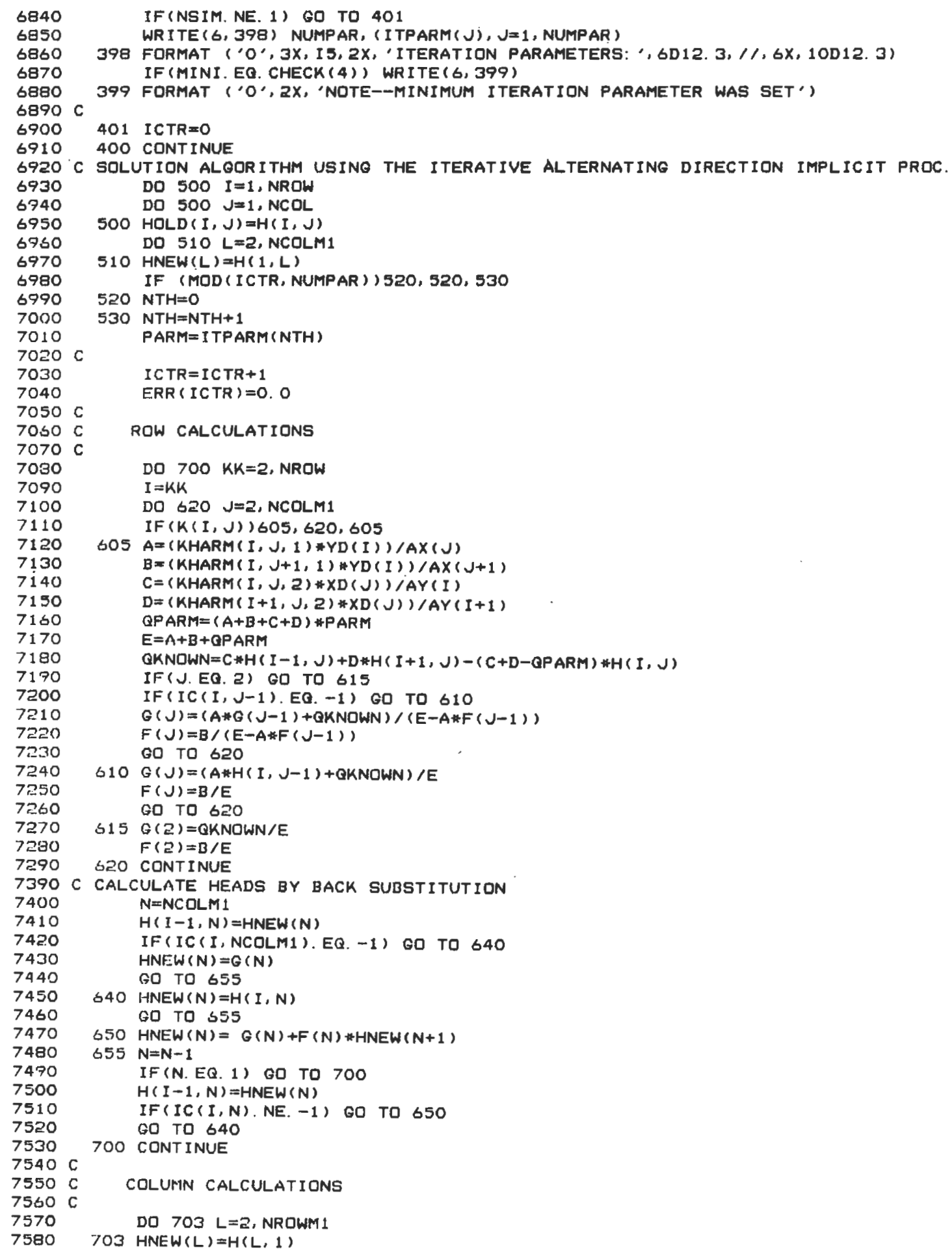




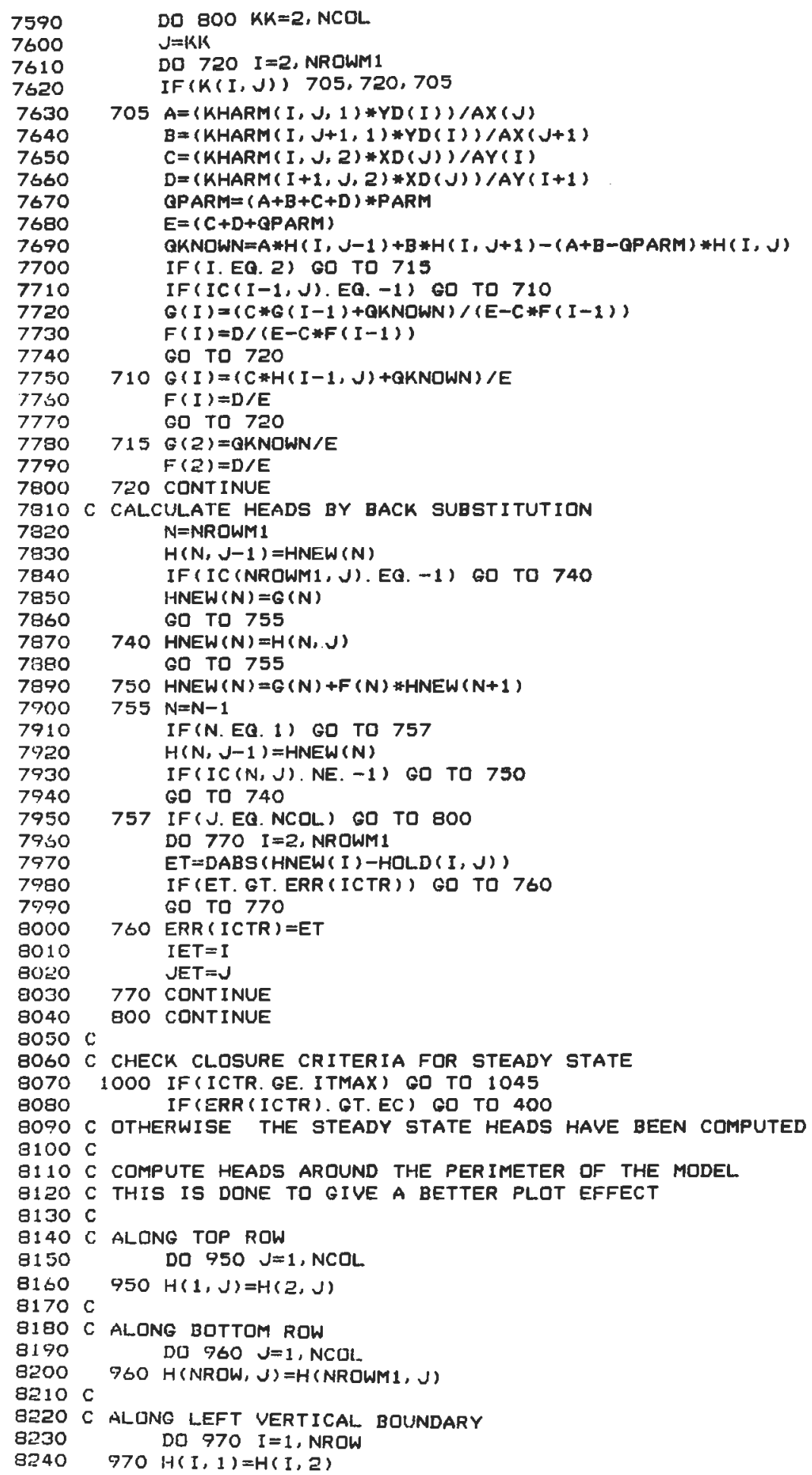




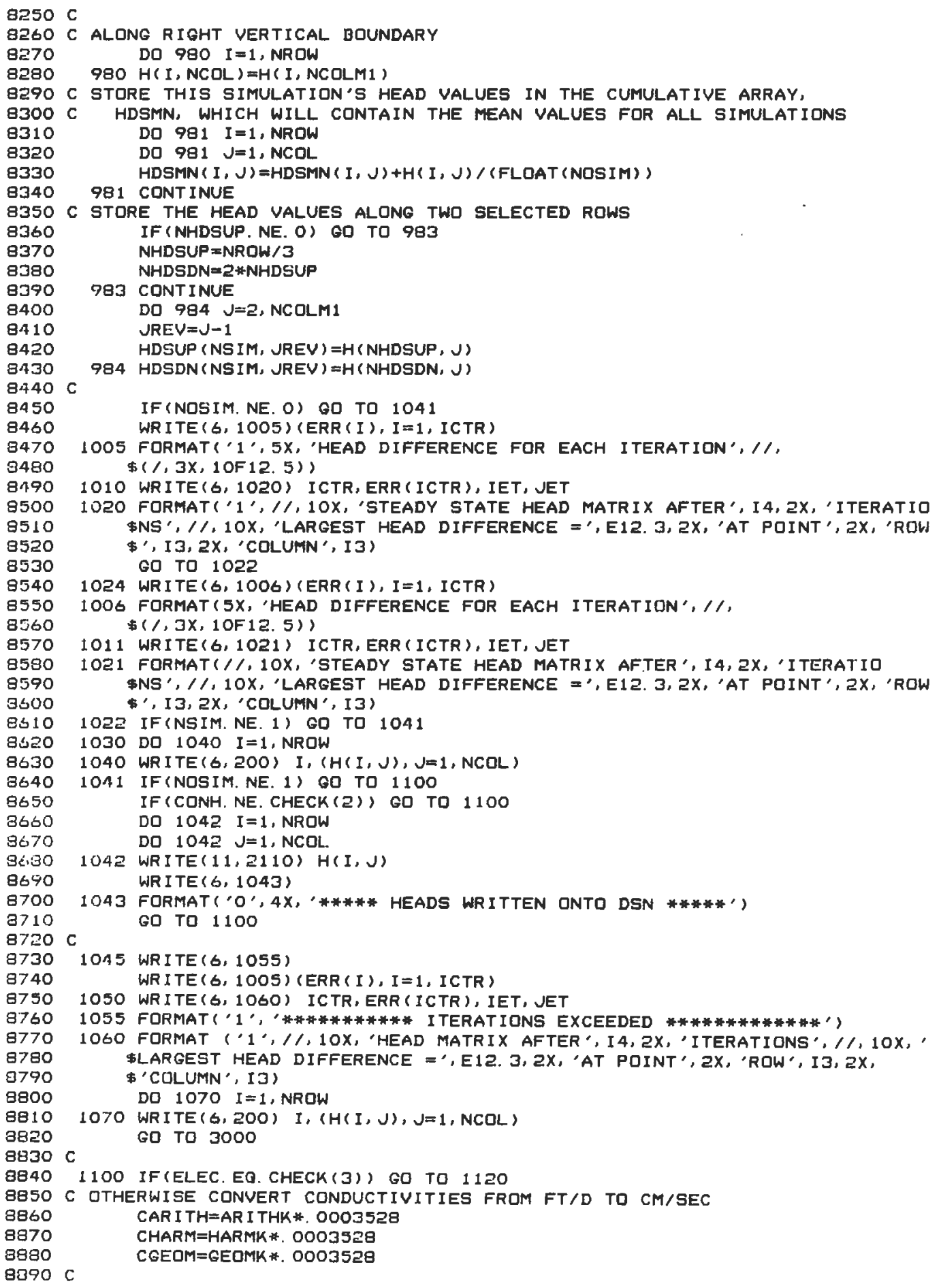


8900

8910

8920

8930

8940

8950

8960

8970

8980

8990

9000

9010

9020

9030

$9040 \mathrm{C}$

$9050 \mathrm{C}$

9050

$9070 \mathrm{C}$

$9080 \mathrm{C}$

9090

9100

9110

9120 .

9130

9140

9150

9160

$9170 \mathrm{C}$

9180

9190

$9200 \mathrm{C}$

$9210 \mathrm{C}$

9250 C

9230 C OTHERWISE THE PREDOMINANT FLOW MUST BE HORIZONTAL

9240 C COMPUTE THE EQUIVALENT HORIZONTAL HYDRAULIC CONDUCTIVITY

$9750 \mathrm{C}$

$9260 \mathrm{C}$

9270

9280

9290

$9300 \mathrm{C}$

9310

9320

9330

9340

9350

CONTINUE

9370 C (DHEAD) IS DISSIPATED

$9380 \mathrm{C}$

$9390 \quad$ LENGTH $=0.0$

$9400 \quad D O 1250 \mathrm{~J}=3, \mathrm{NCOLM1}$

$9410 \quad$ LENGTH=LENGTH+AX (J)

$9420 \quad 1250$ CONTINUE

$9430 \mathrm{C}$

9440 C KHEQFD=EQUIVALENT HORIZONTAL HYDR. COND. IN UNITS OF FT. /DAY

$9450 \quad K H E Q F D=(Q * L E N G T H) /(D H E A D * A R E A)$

$9460 \quad$ HCSTAT $(N S I M, 6)=K H E Q F D$

9470 C KHEQCS=EQUIVALENT HORIZONTAL HYDRAULIC CONDUCTIVITY IN UNITS OF CM. /SEC.

$9430 \quad K H E Q C S=K H E Q F D * 0003528$

9490 IF (ELEC. EQ. CHECK (3)) GO TO 1290

$9500 \mathrm{C}$

9510 C OTHERWISE WE HAVE THE hYDRAULIC CASE

95201280 FORMAT $(' 0 \prime, / / 1 / 1,5 X$, 'MACROSCOPIC PARAMETERS', $/ 1,8 X$, 'EQUIVALENT ',

9530

9540

9550

9560

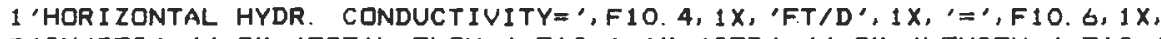

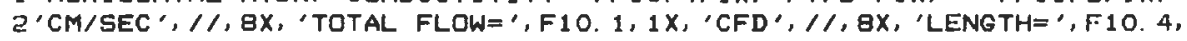
$31 X, ' F T ', / /, 8 X$, 'AREA=',FIO. 4, $1 X$, 'SO. FT. ', //, BX, 4'TOTAL DISSIPATED HEAD=',F10. 4, 1 X, 'FT.', 


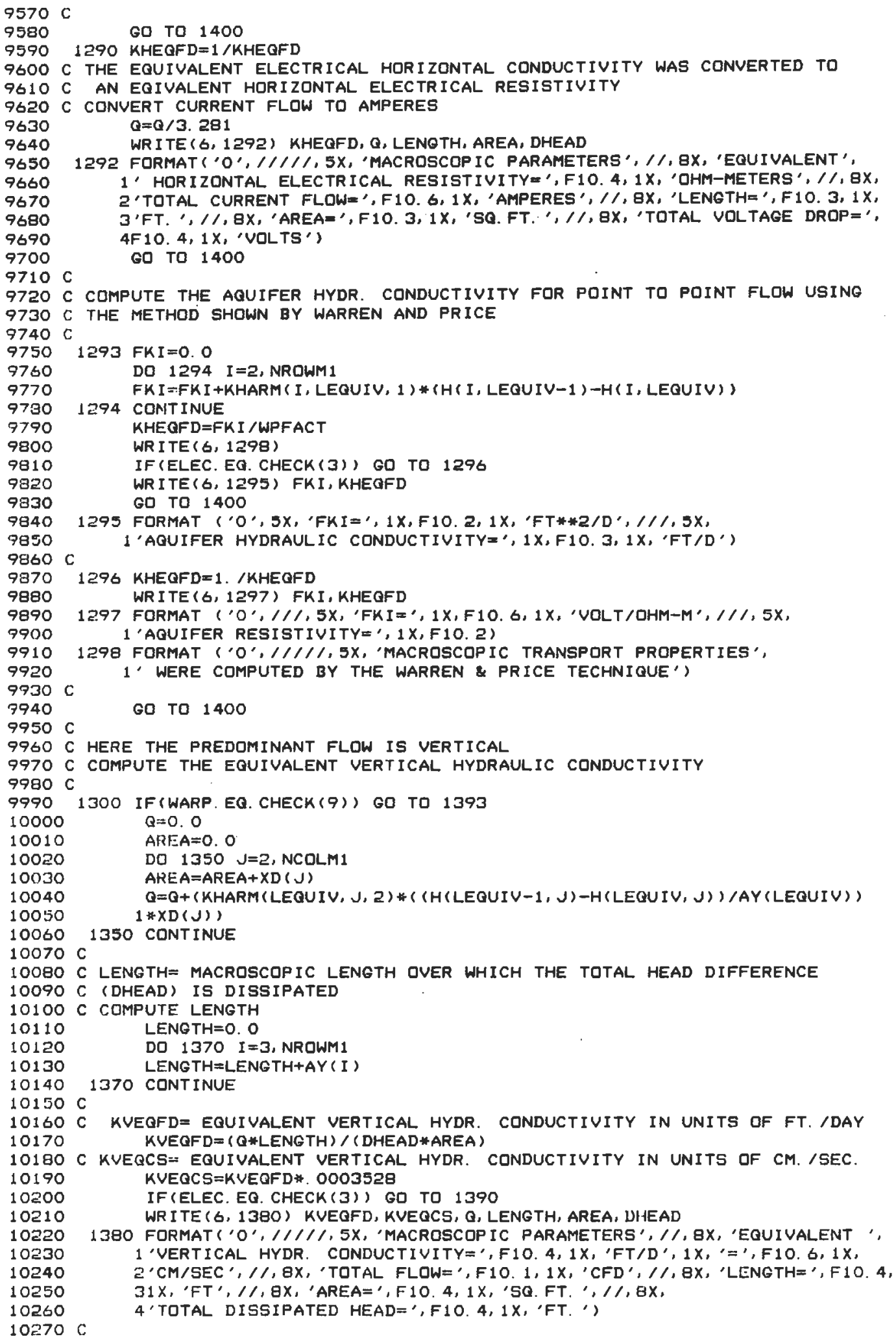




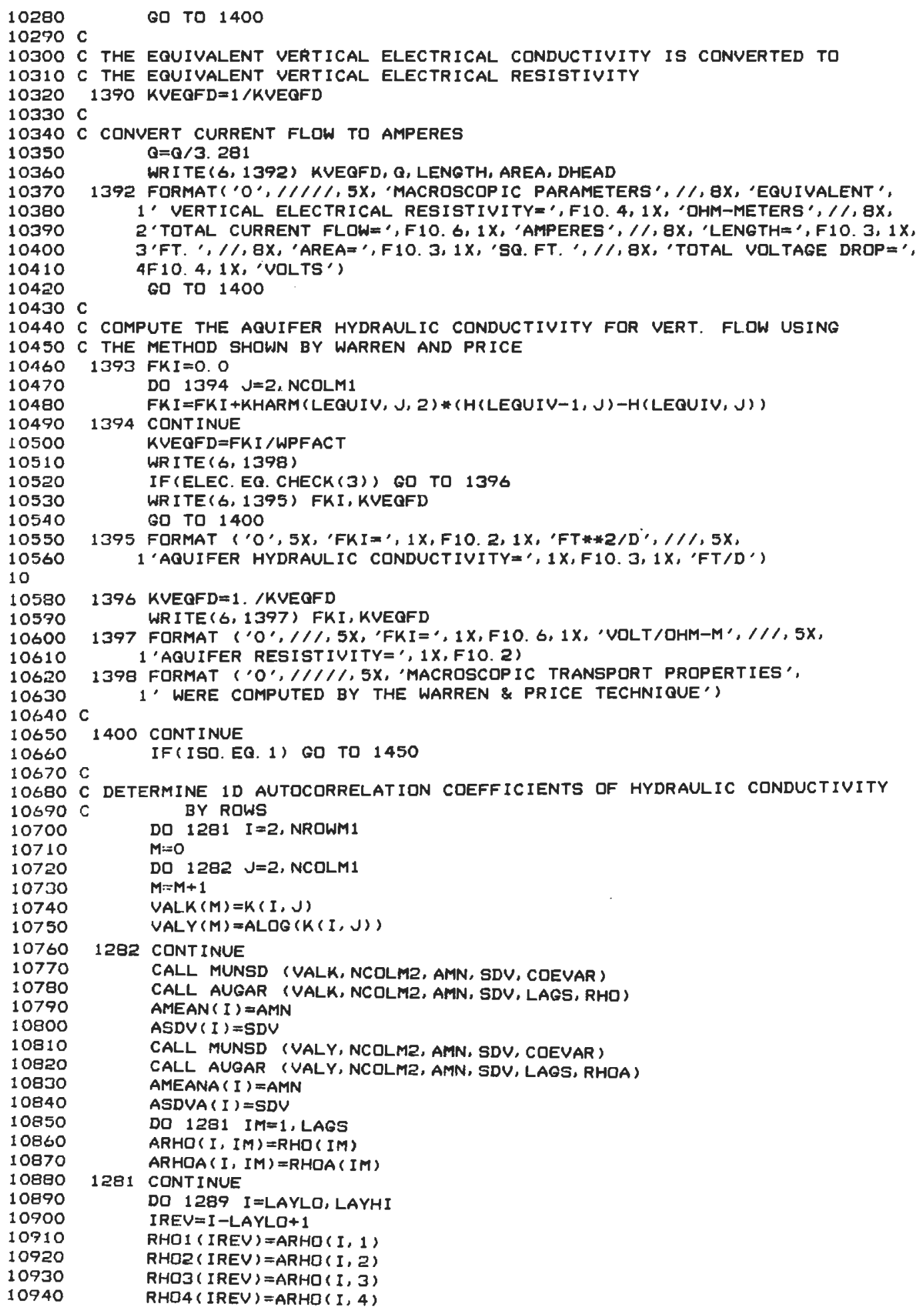




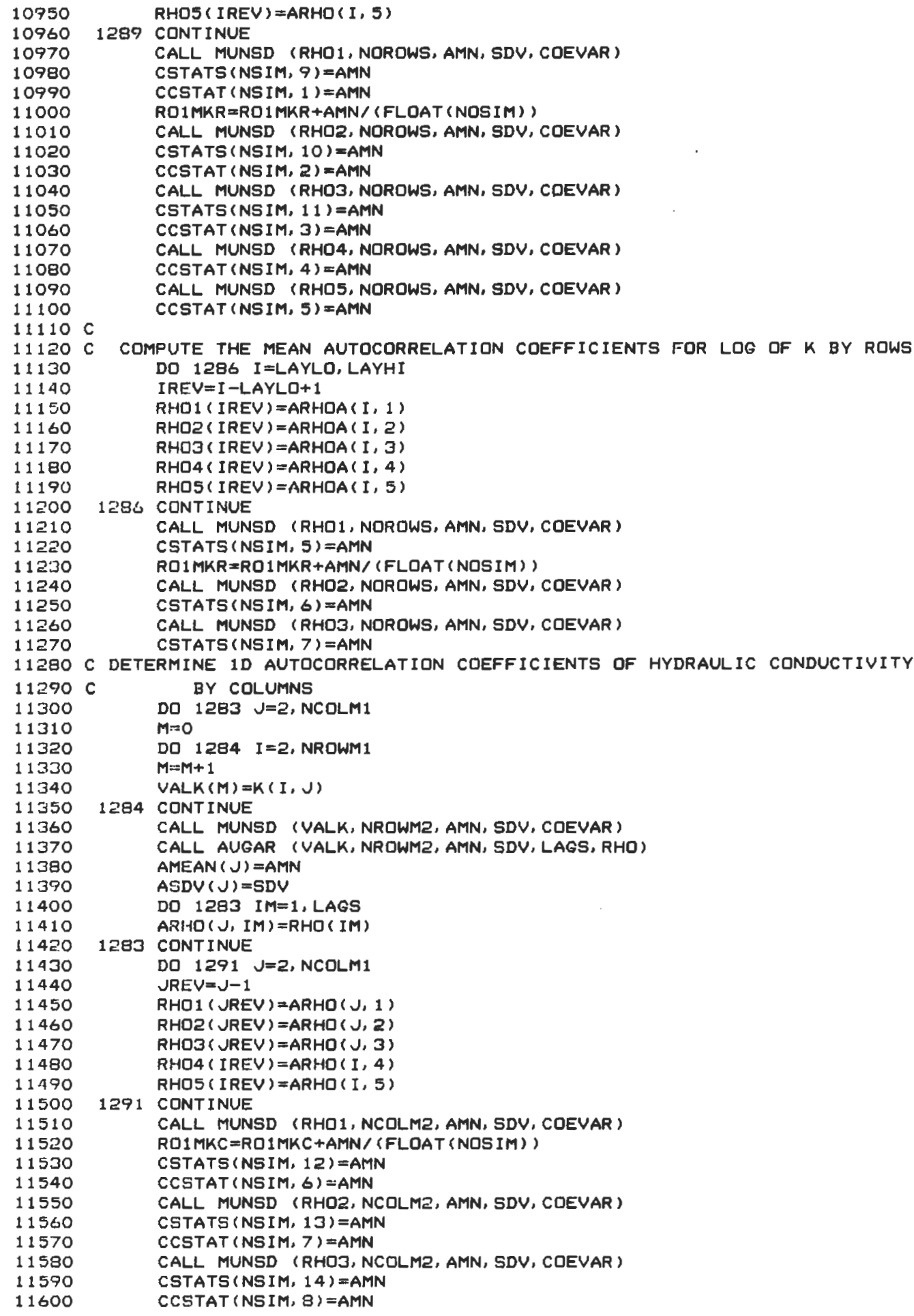




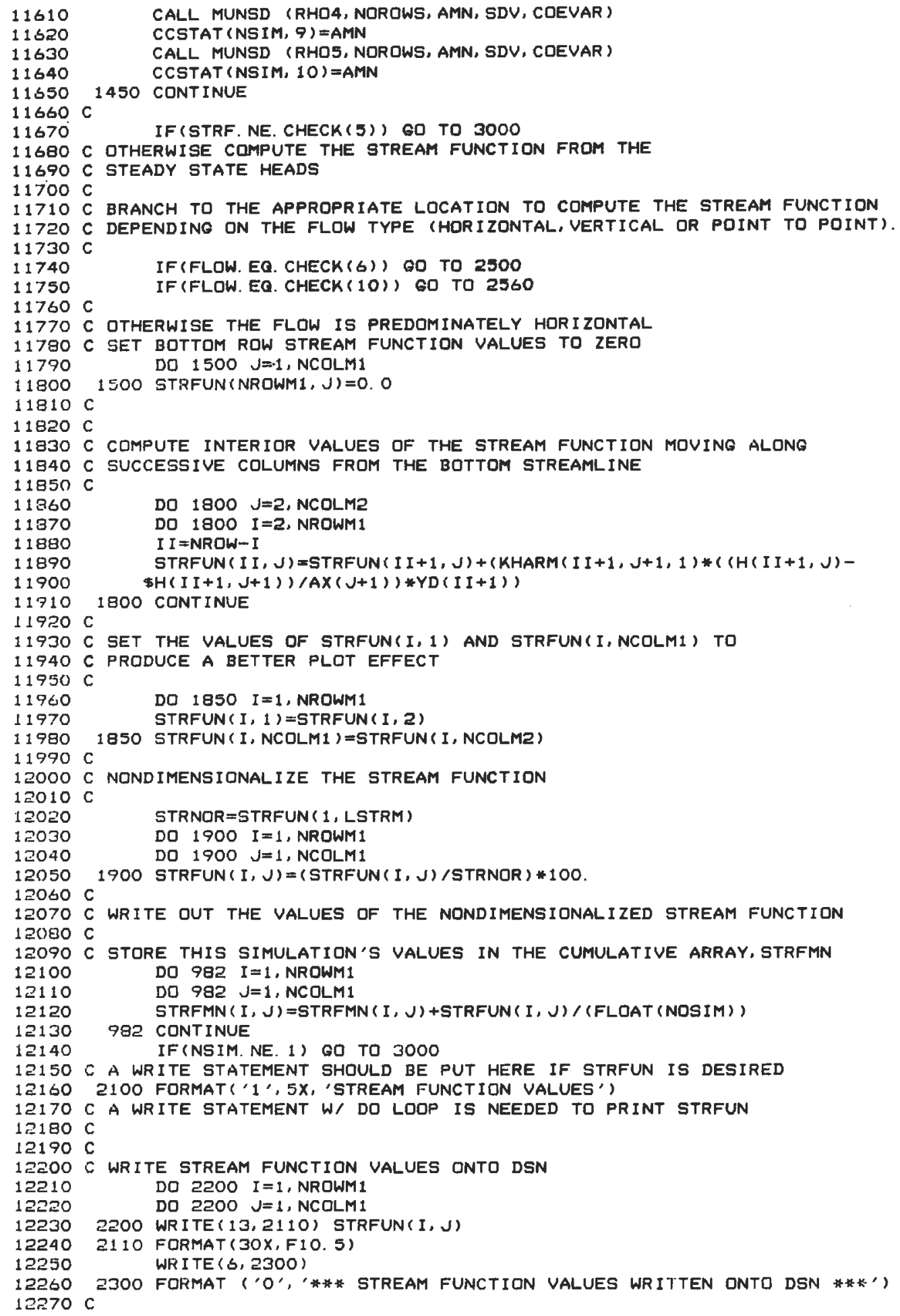




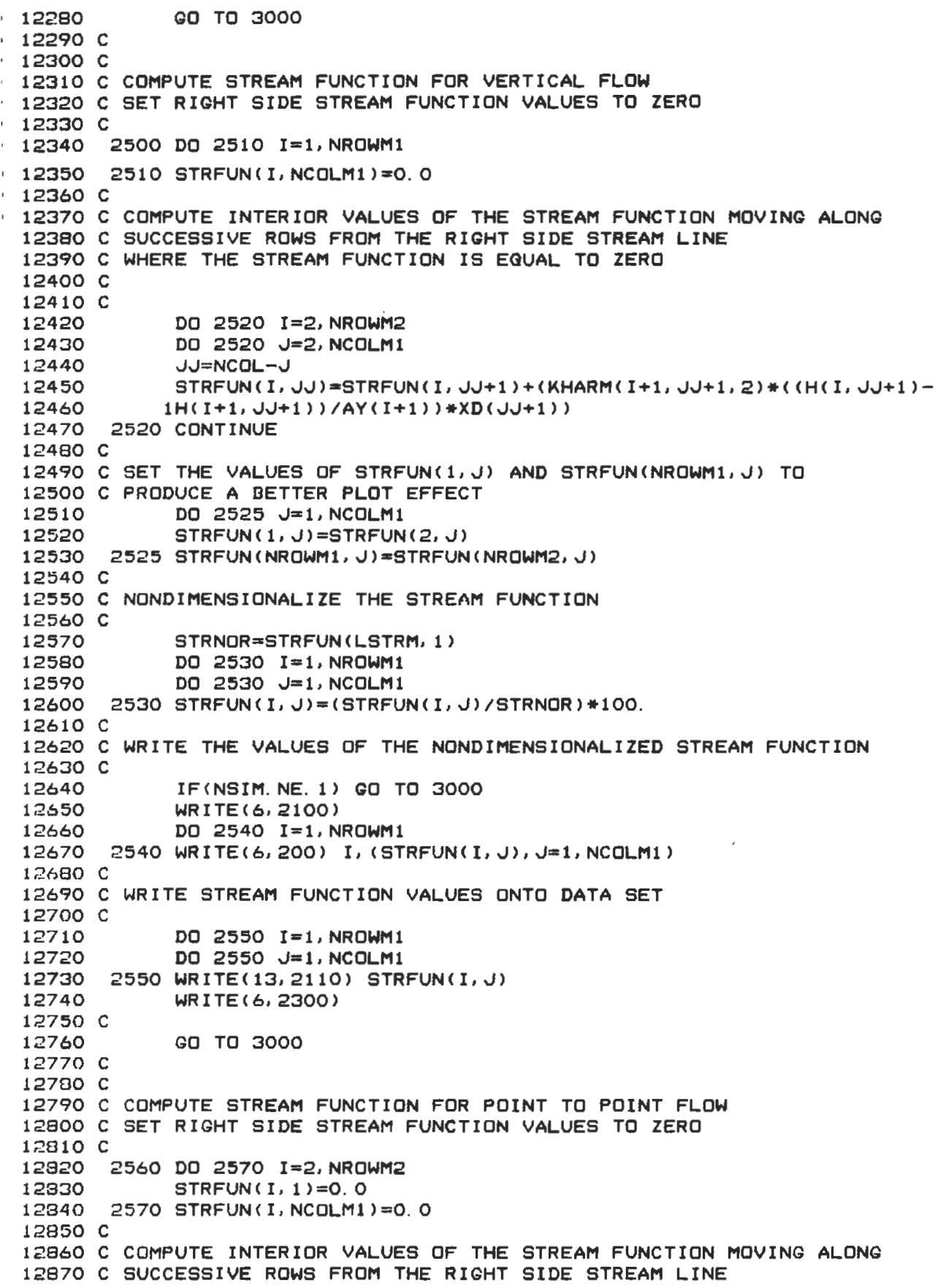




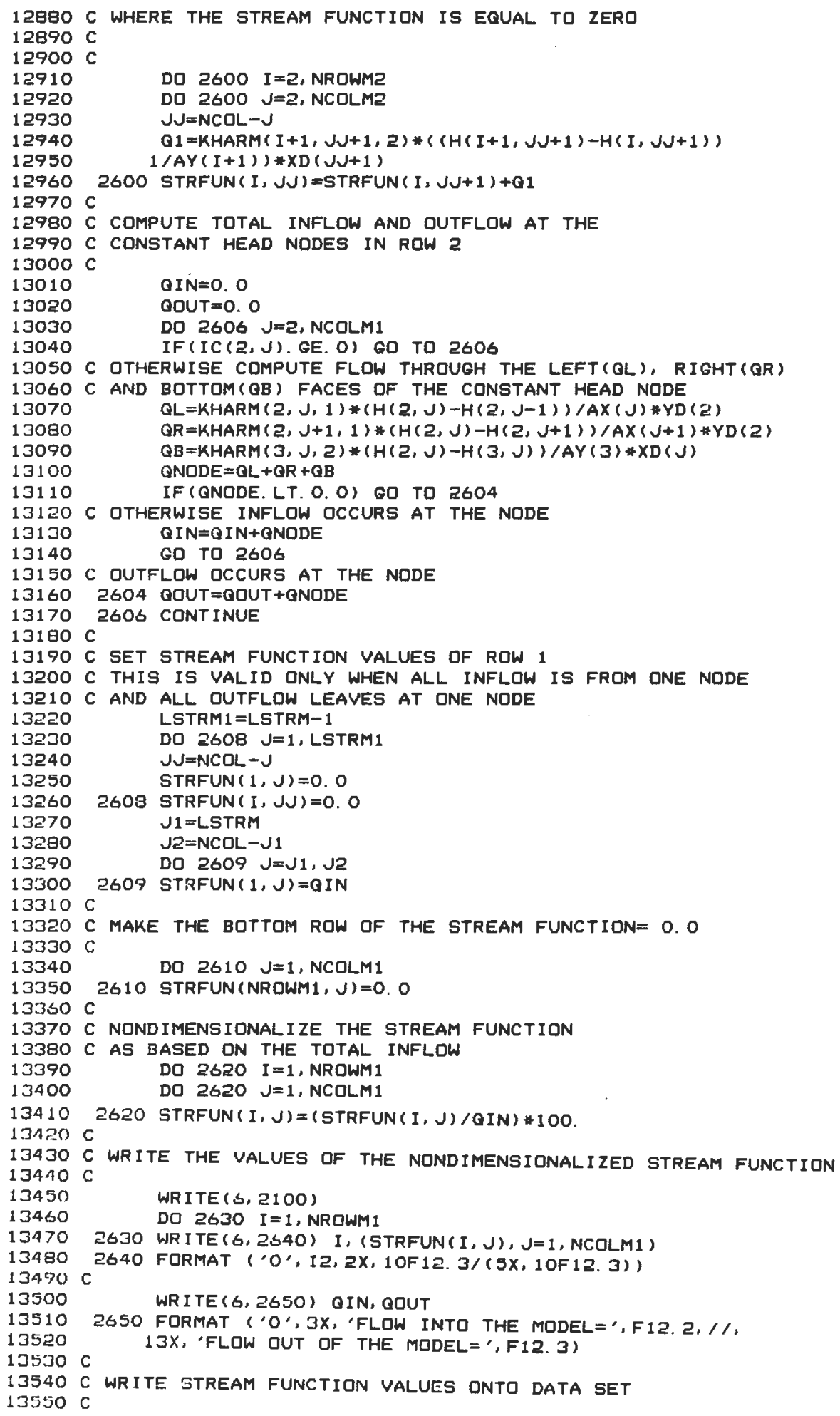




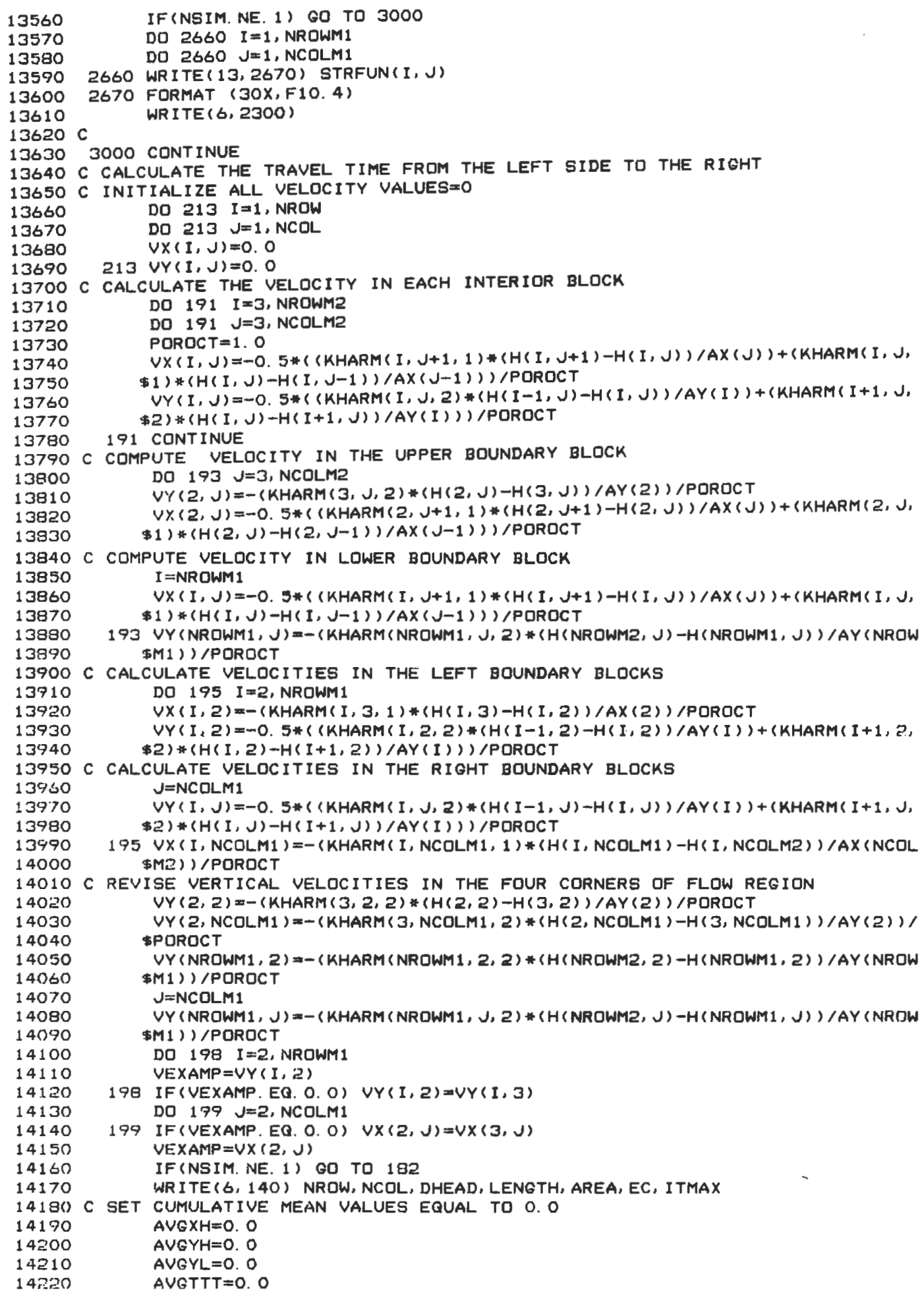




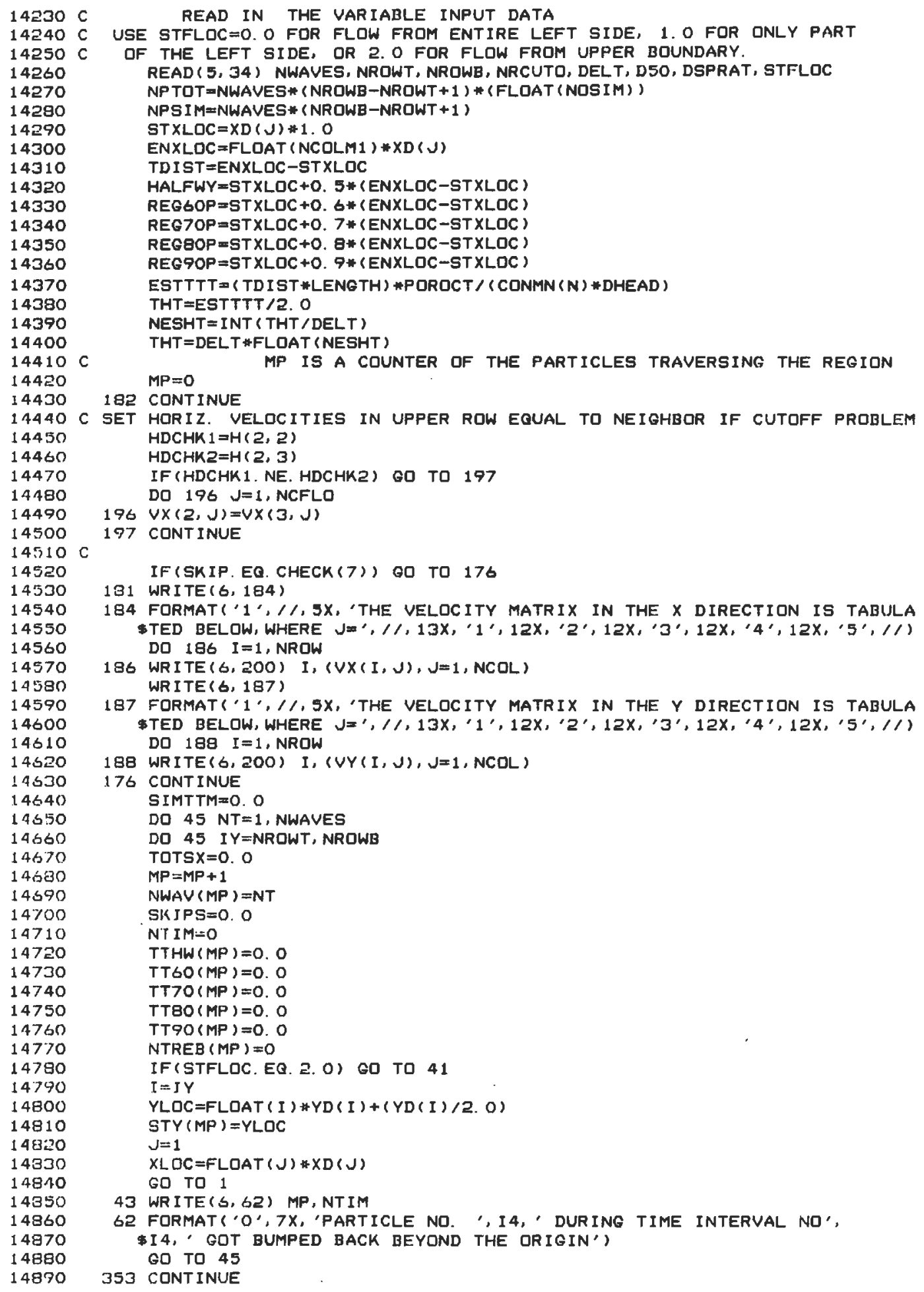




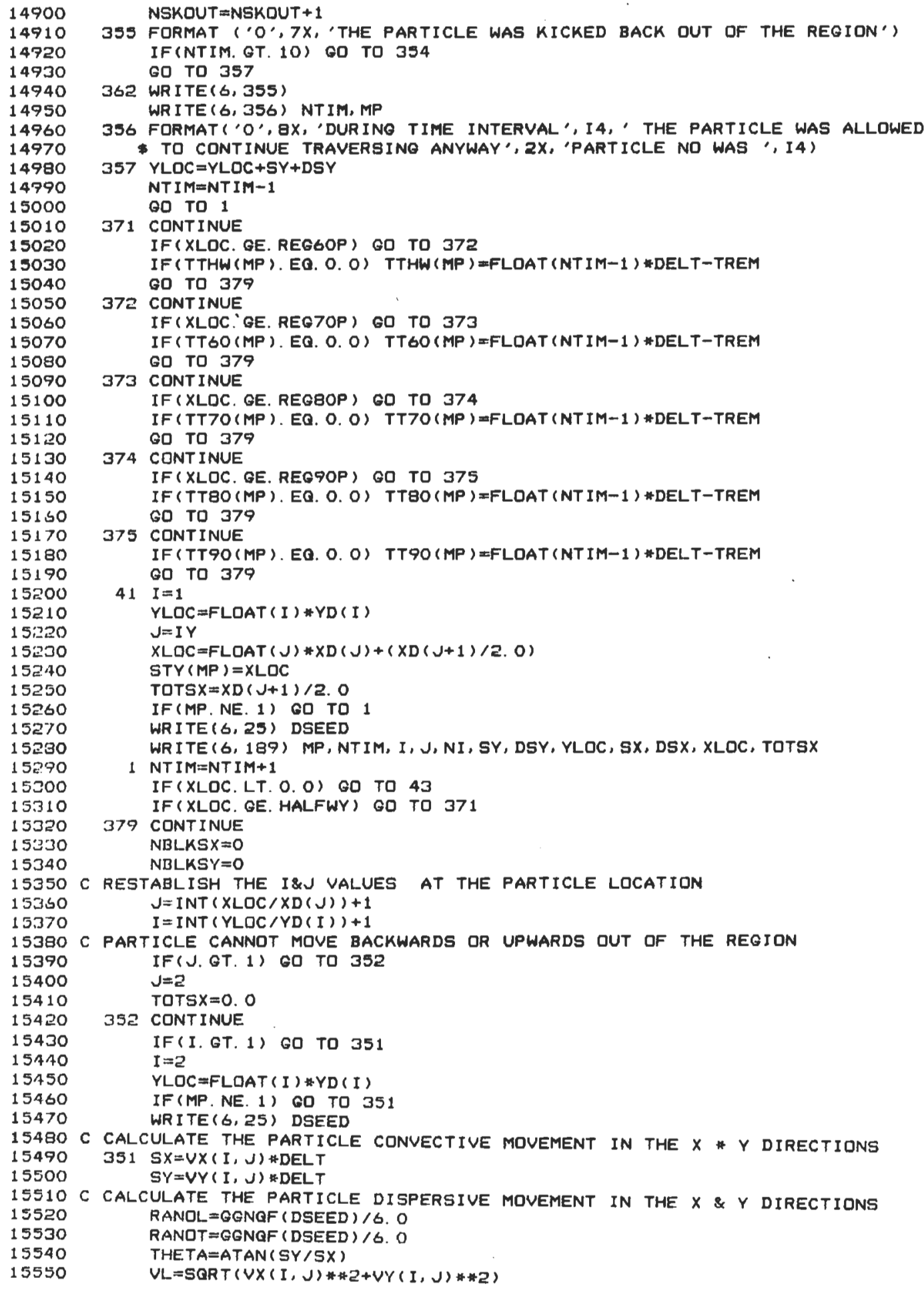




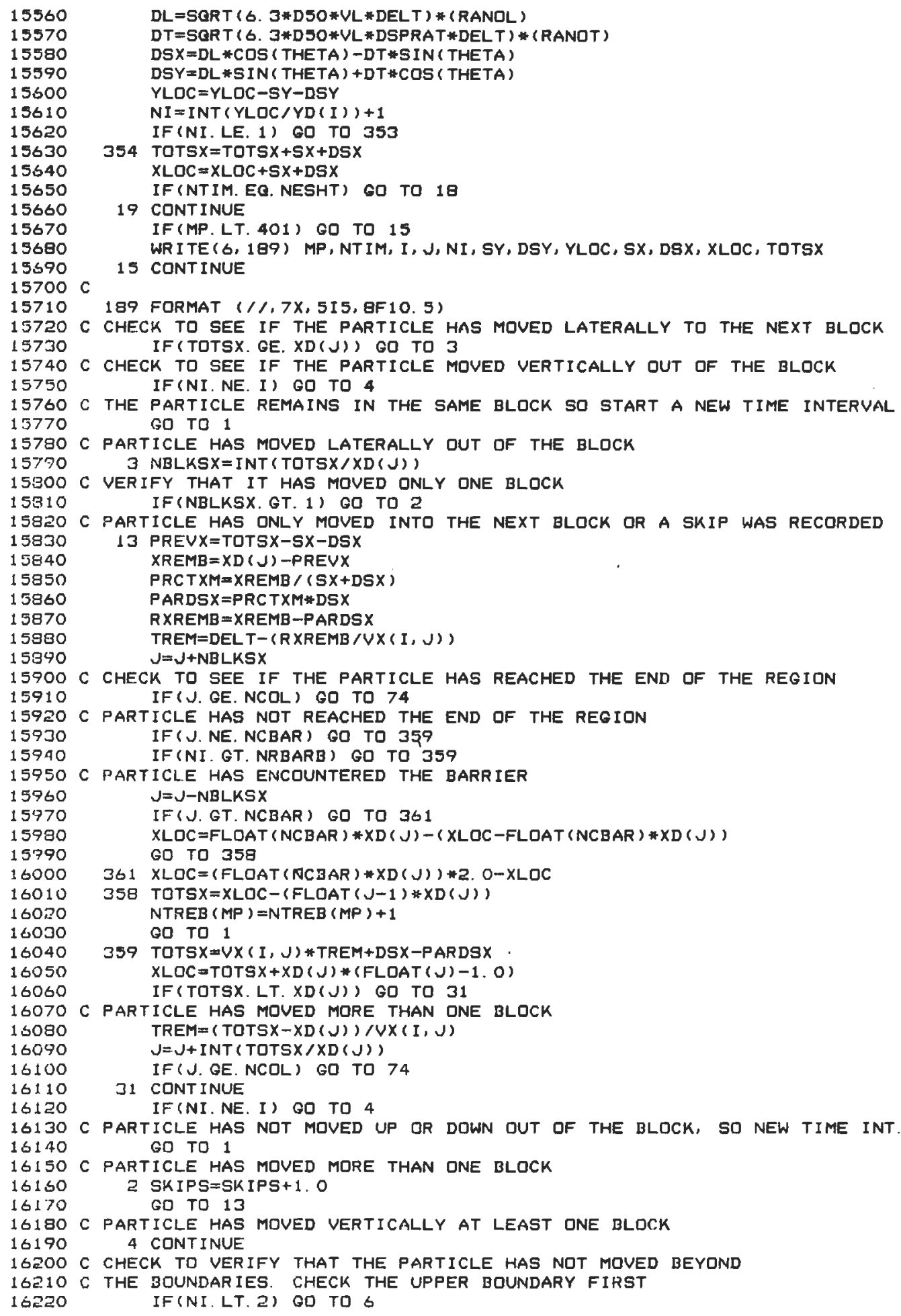




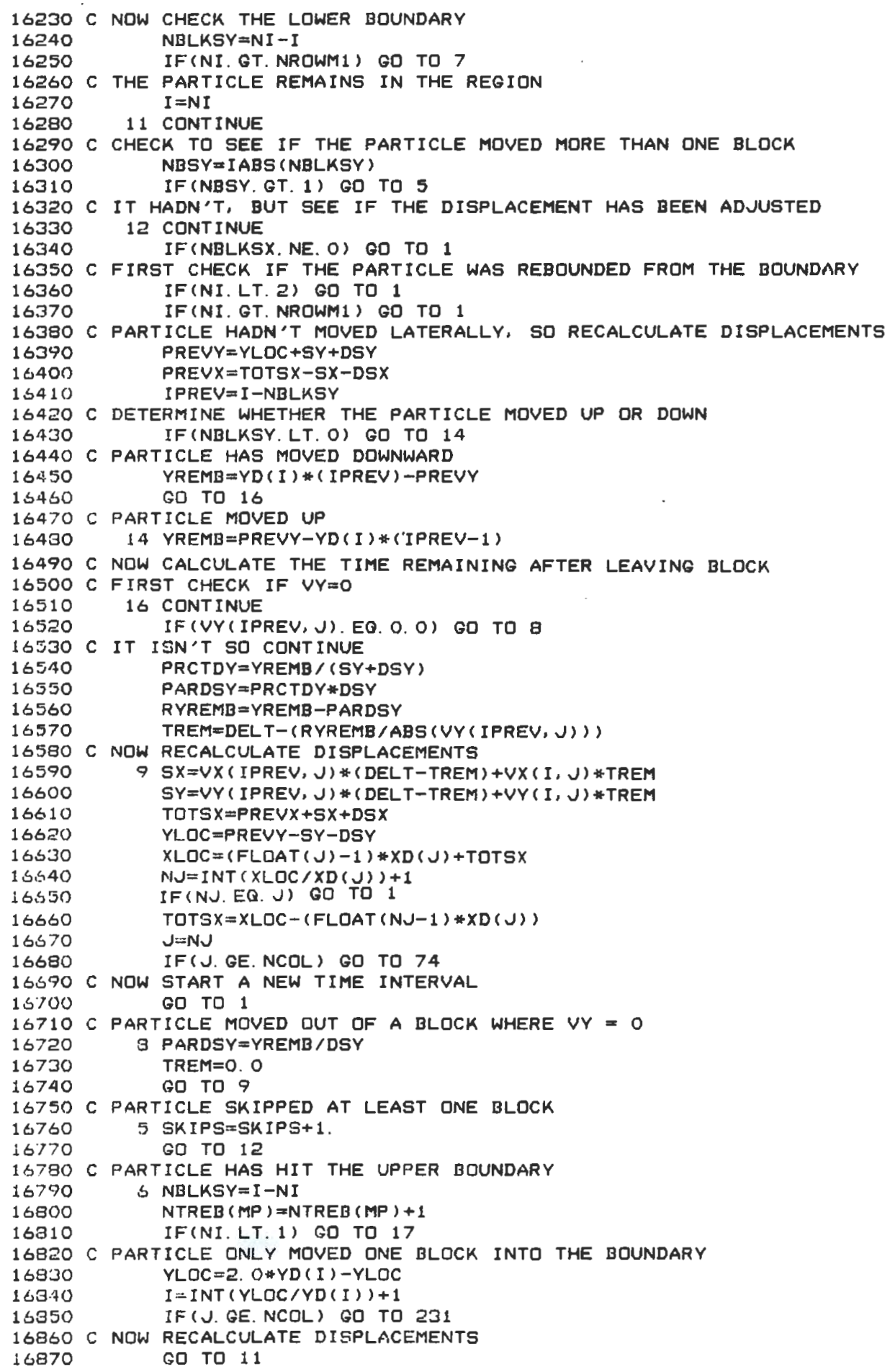




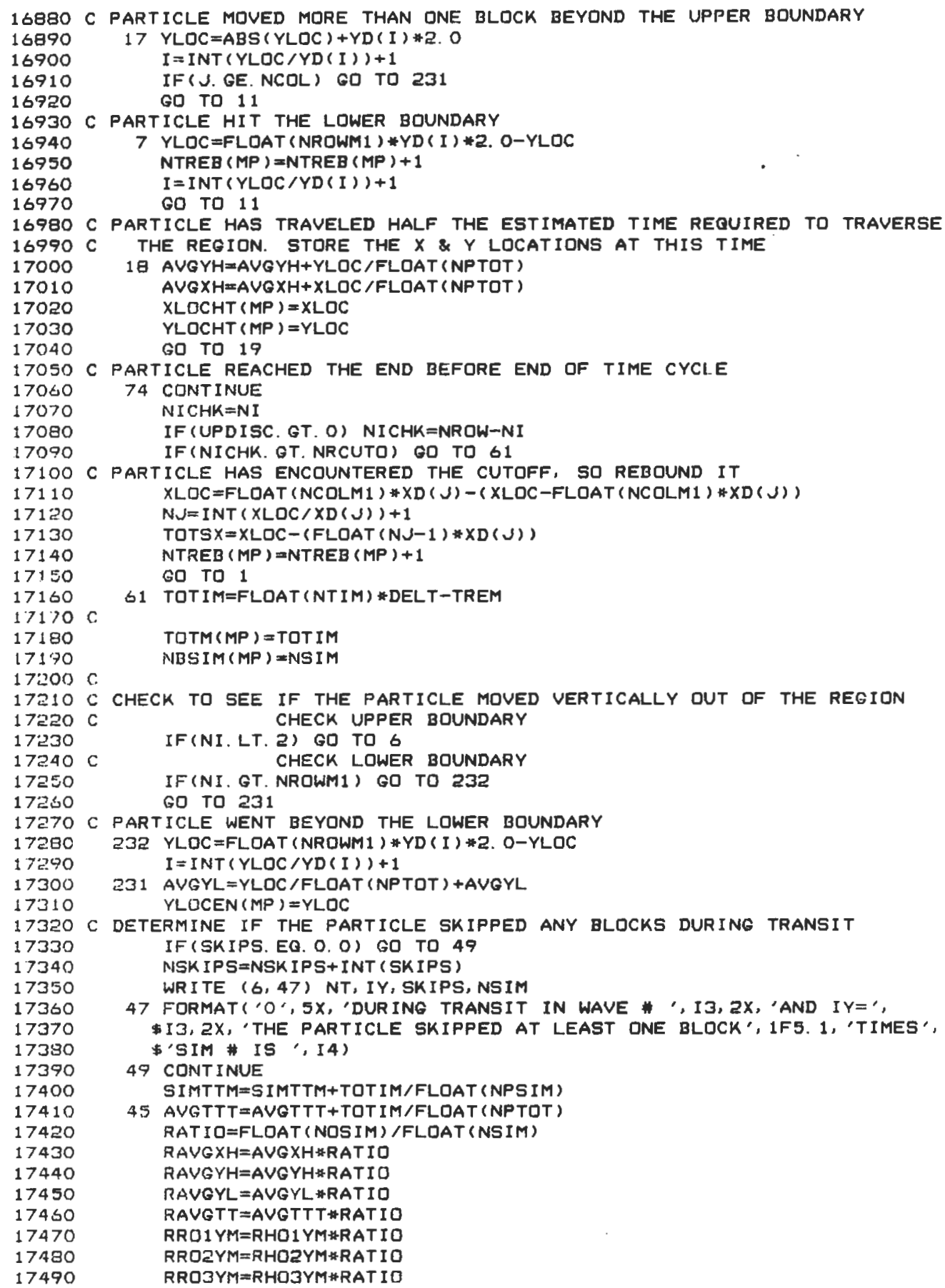




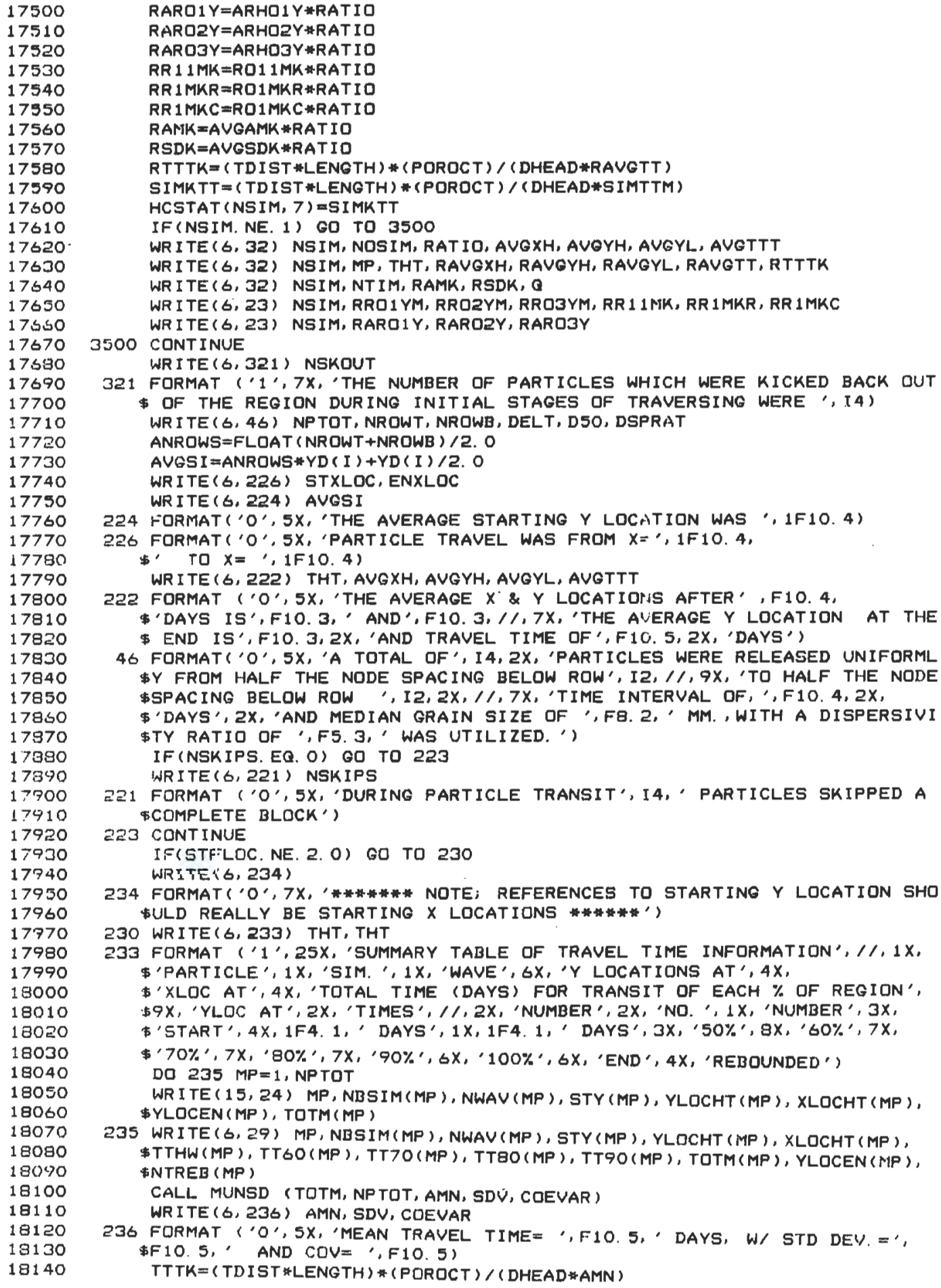


18360

18370

18330

18390

18400

13410

13420

19430

18440

18450

134.50

13470

18480

18490

18500

18510

1 घ520

18530

13540

13550

1 घ560

13570

18580

18590

18500

18610

18620

18630

18640

18650

18660

18670

18680

18690

18700

19710

18720

18730

13740

19750

18760

18770

19730

18790 18800
WRITE (6, 238) TTTK

238 FORMAT' $' 0$ ', 5X, 'EFFECTIVE HYDRAULIC CONDUCTIVITY OF THE REGION ON

\$BASIS OF TRAVEL TIME IS ',F10. 5, "FT/DAY')

CALL MUNSD (YLOCEN, NPTOT, AMN, SDV, COEVAR)

WRITE (6, 237) AMN, SDV, COEVAR

237 FORMAT $(' 0 \%, 5 X$, 'MEAN Y COORDINATE AT END OF TRANSIT IS', F10.4,

\$'WITH STD DEV =",F10. 5," AND COV = "F10.6)

WR ITE (6, 239) NDSIM, AVGAMK, AVGSDK

239 FORMAT $(' 0 \%$ '5X, MEAN OF HYDRAUL IC CONDUCTIVITY FOR A TOTAL OF ',

\$I4,' SIMULATIONS IS ',F10. 4,' WITH A MEAN STD. DEV. OF ',F10. 4)

C WRITE OUT THE MEAN VALUES OF THE HEAD MATRIX COMPUTED FOR ALL THE

SIMULATIONS

ADD A WWR ITE STATEMENT HERE TO DO THAT

C CALCULATE MEAN \& STD DEV. OF THE HEADS FOR ALL SIMS AT PRESELECTED SPOTS DO $263 \mathrm{~J}=1, \mathrm{NCOLM2}$

$M=0$

DO $264 I=1, N O S I M$

$M=M+1$

$\operatorname{VALK}(M)=\operatorname{HDSUP}(I, J)$

264 CONTINUE

CALL MUNSD (VALK, NDSIM, AMN, SDV, COEVAR)

$\operatorname{AMEAN}(J)=A M N$

$\operatorname{ASDV}(J)=S D V$

263 CONTINUE

DO $265 \mathrm{~J}=1, \mathrm{NCOLM} 2$

$M=0$

DO $266 I=1$, NOSIM

$M=M+1$

$\operatorname{VALK}(M)=\operatorname{HDSDN}(I, J)$

266 CONTINUE

CALL MUNSD (VALK, NOSIM, AMN, SDV, COEVAR)

AMEANA $(J)=A M N$

$A S D V A(J)=S D V$

265 CONTINUE

WR ITE (6, 269) NHDSUP, NHDSDN, NCOLM1

DO $267 \mathrm{I}=1, \mathrm{NOSIM}$

WRITE $(6,200) \quad I,(\operatorname{HDSUP}(I, J), J=1, \operatorname{NCOLM2})$

267 WRITE $(20,205)$ I, $(\operatorname{HDSUP}(I, J), J=1, \operatorname{NCOLM} 2)$

DO $268 I=1, N O S I M$

GO TO 268

261 WRITE $(6,200)$ I, (HDSDN $(1, J), J=1$, NCOLM2)

2S日 WRITE $(21,205)$ I , (HDSDN $(I, J), J=1, \operatorname{NCOLM2)}$

241 CONT INUE

WR I TE (6,271) NHDSUP, NHDSDN, NHDSUP, NHDSDN

DO $272 J=1$, NCOLM2

VARHUP $=($ ASDV $(J)) * * 2$

VARHDN $=($ ASDVA $(J)) * 2$

JREV $=J+1$

WR I TE (22, 23 ) JREV, AMEAN ( $)$, ASDV ( $)$, VARHUP, AMEANA ( $J), A S D V A(J), V A R H D N$

272 WRITE $(6,23)$ JREV, AMEAN $(J), \operatorname{ASDV}(J), V A R H U P, \operatorname{AMEANA}(J), A S D V A(J), V A R I D N$

271 FORMAT $\left(' 0 \prime, 7 x_{1}\right.$ ' TABLE OF MEAN AND STD. DEV. OF HYD HEADS FOR THE

\$TW PRESELECTED ROWS, ', I 4,' AND', $14,1 /, 5 X$, 'COLUMN', ?X,

\$'FOR HEADS IN ROW ', I $4,7 x$, 'FOR HEADS IN ROW ', I 4, 11, 14X, MEAN',

$\$ 4 X$, 'STD. DEV. ', $2 X$, 'VARIANCE', 3X, 'MEAN', 4X, 'STD. DEV. VARIANCE',

269 FORMAT $\left(' 1 \%, 7 X_{2}\right.$ 'THE HEAD VALUES FOR EACH SIMIULATION FOR ROWS',

- I4,' AND ', 14,' LISTED BELOW BY COLUMN', $11,2 x$, 'SIMULATION', $2 x$,

\$'COLUMN FROM 2 THROUGH ', I4)

IF (CONH. NE. CHECK(2)) GO TO 242

DO $242 I=1$, NROW

DO $242 J=1, N C O L$

WR ITE $(11,204) \operatorname{HDSMN}(I, J)$

242 CONT INUE

240 FORMAT $(1 \%, 1 / 10 \mathrm{X}$, 'MEAN STEADY STATE HEAD MATRIX FOR $'$ I 4

"' SIMULATIONS',

C WRITE DUT THE MEAN STREAM FUNCTION VALUES AND TO A DISK IF CALCULATED IF (STRF. NE. CHECK (5)) GO TO 245 


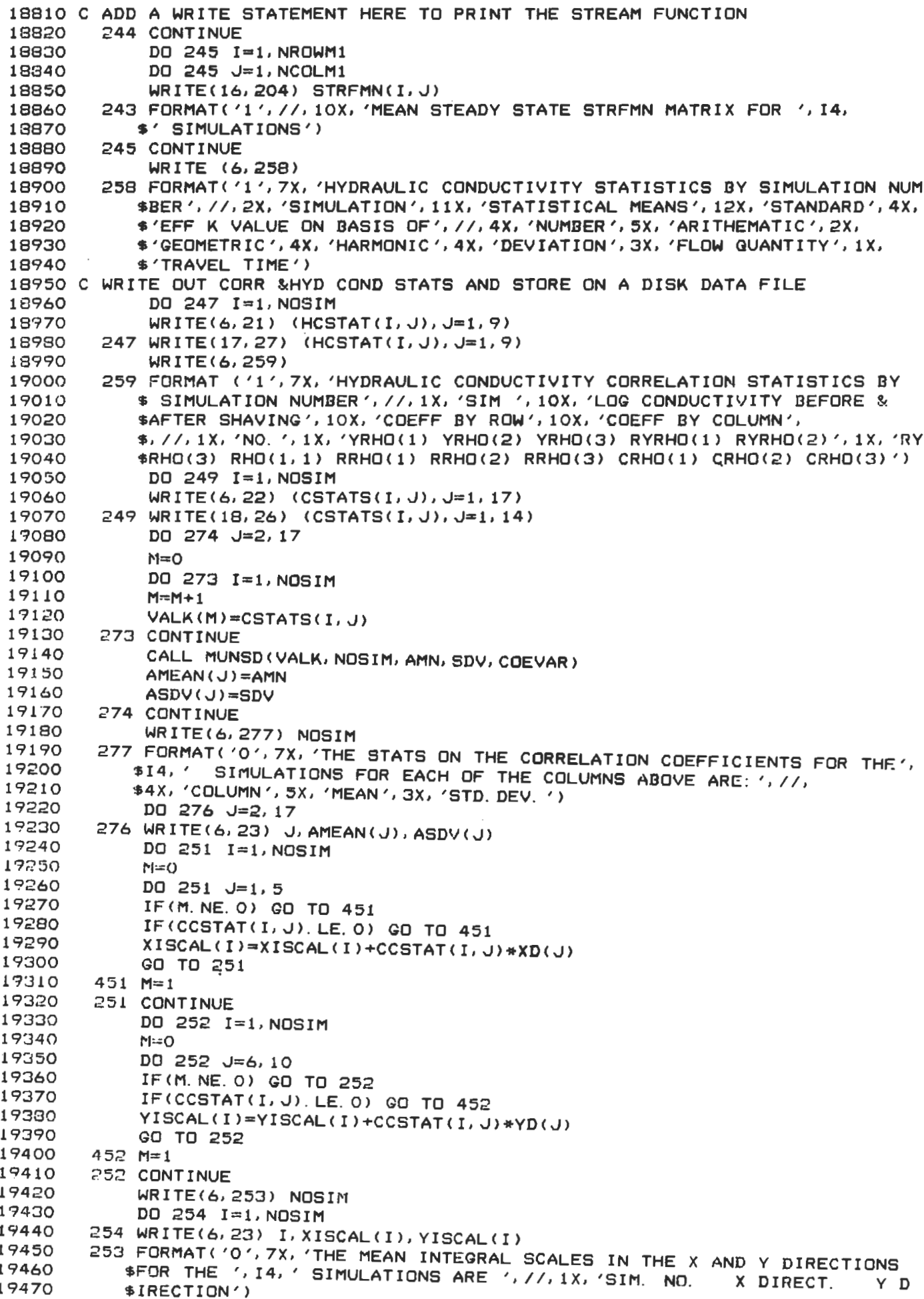




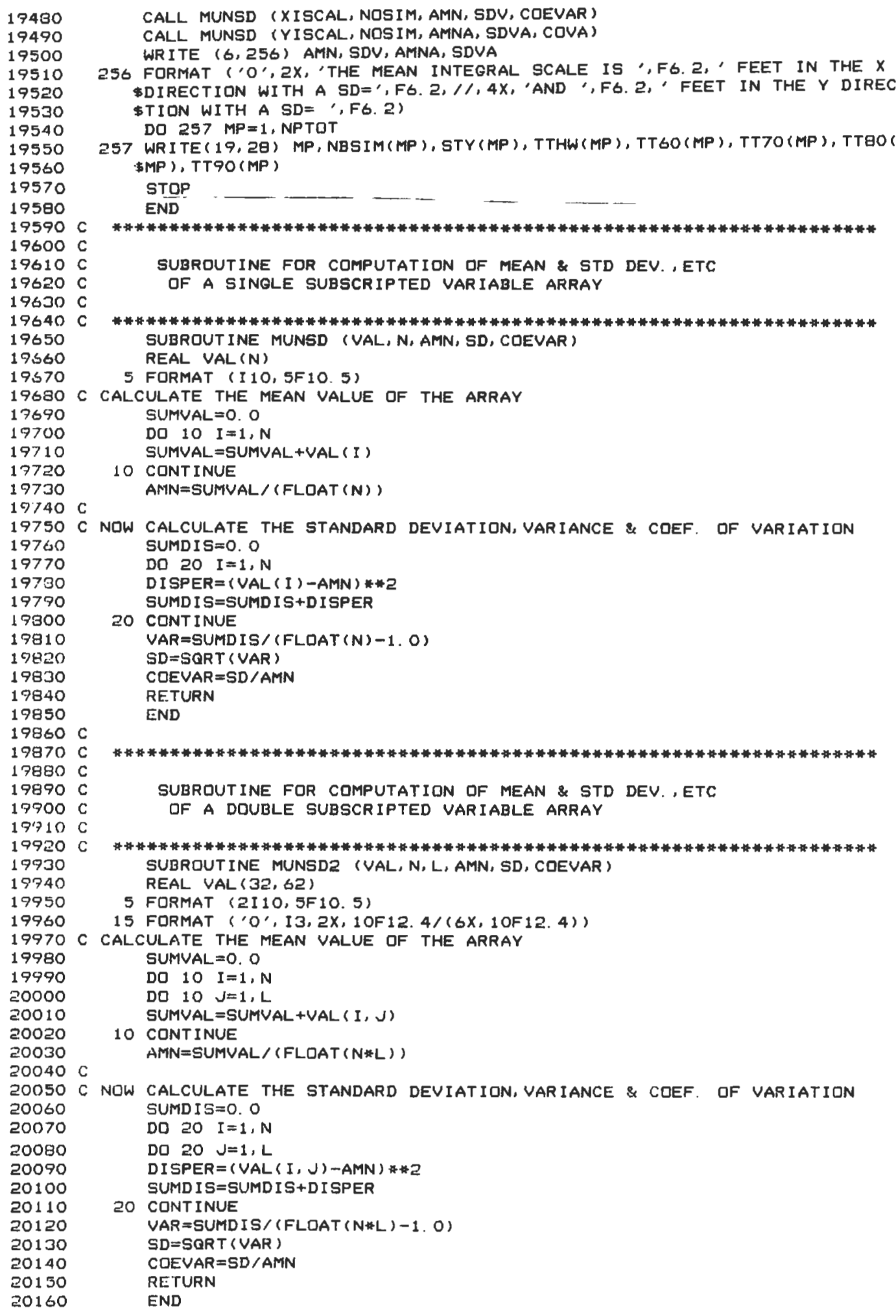




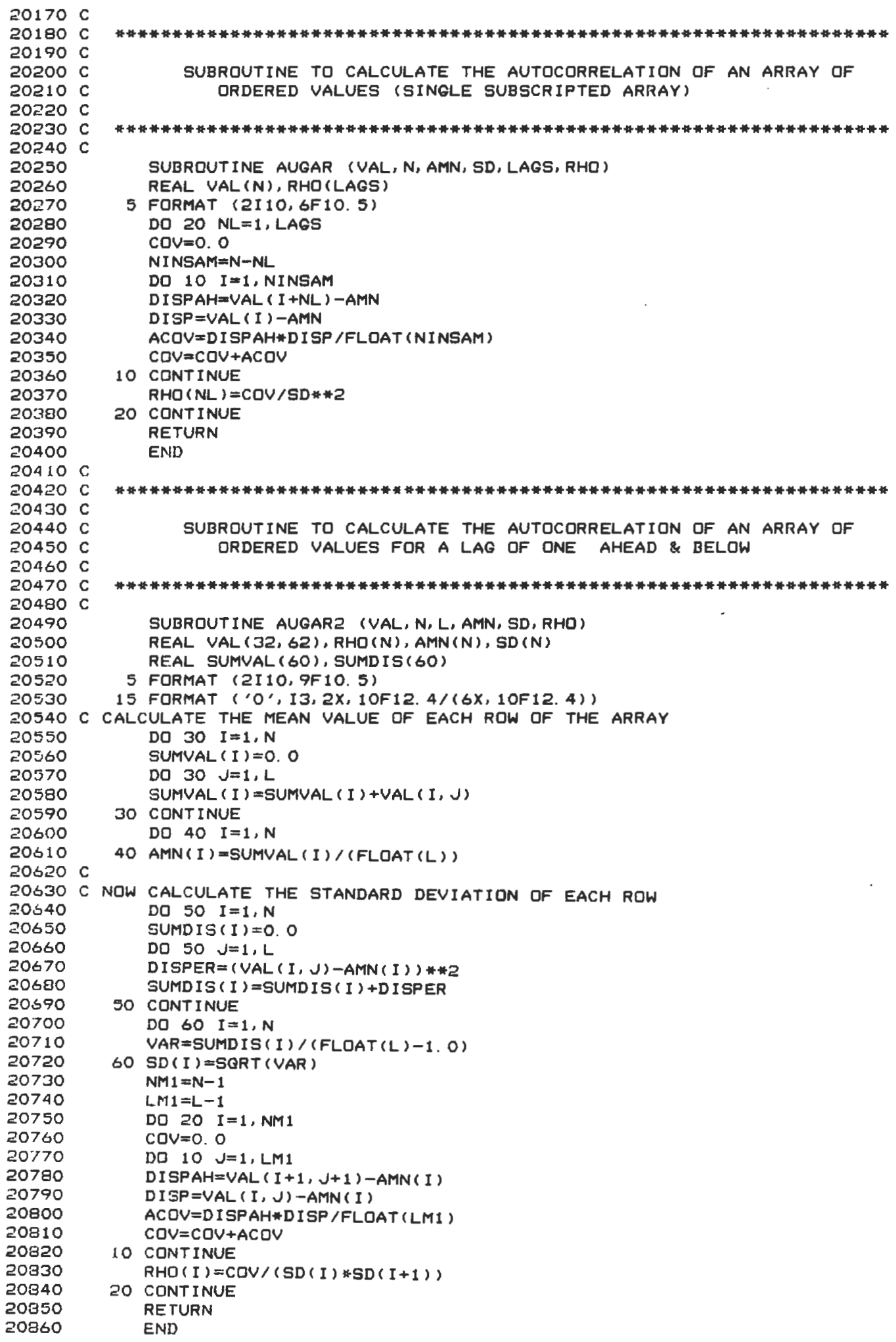

